DESY behălt sich alle Rechte für den Fall der Schutzrechtserteilung und für die wirtschaftliche Verwertung der in diesem Bericht enthaltenen Informationen vor.

DESY reserves all rights for commercial use of information included in this report, especially in case of filing application for or grant of patents.

To be sure that your preprints are promptly included in the HIGH ENERGY PHYSICS INDEX,

send them to the following address (if possible by air mail):

DESY

Bibliothek

Notkestrasse 85

2 Hamburg 52

Germany 


\section{Selberg Supertrace Formula for Super Riemann Surfaces, Analytic Properties of Selberg Super Zeta-Functions and Multiloop Contributions for the Fermionic String}

Christian Grosche II.Institut für Theoretische Physik, Universität Hamburg
Luruper Chaussee 149,2000 Hamburg 50 Fed.Rep.Germany

\section{Abstract:}

In this paper I present a complete derivation of the Selberg supertrace formula for super Riemann surfaces and a discussion of the analytic properties of the Selberg super zeta-functions.

The Selberg supertrace formula is based on Laplace-Dirac operators $\square_{m}$ of weight $m$ on super Riemann surfaces. The trace formula for all $m \in \mathbf{Z}$ is derived and it is shown that one nust discriminate between even and odd $m$. Particularly the term in the trace formula proportional to the identity transformation is sensitive to this discrimination.

Furthermore the analytic properties of the two Selberg super zeta-functions are discussed in detail; first with. and the second without consideration of the spin structure. As it is shown the Selberg super zeta-functions have a similar zero structure as the ordinary Selberg zeta-function. Also, functional equations for the two Selberg super zeta-functions are derived.

I apply my results to discuss the spectrum of the Laplace-Dirac operators and to calculate their determinants. For the spectrum I find that the nontrivial Eigenvalues are the same for $\square_{m}$ and $\square_{0}$ up to a constant depending on $m$. which is analogons to the bosonic case.

The analytical properties of the determinants can be deduced from the analytical properties of the Selbere super zeta-functions, and it is shown that they are wellrefined. Special cases $(m=0.2)$ for the determinants are important in the Polyakor approach for the fermionic string. With these results it is deduced that the fermionic string integrand of the Polyakov functional integral in well-defined.

A short introduction into the theories of supermanifolds. super Riemann surfaces and the Poincare super upper half-plane is incluiled.
II. Super Riemann Surfaces and Super Uniformisation

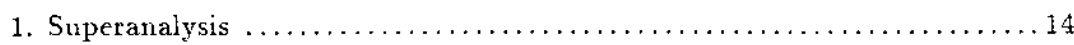

2. Supermanifolds 19

III. Physics on the Poincaré Super Upper Half-Plane $S \mathcal{H}$

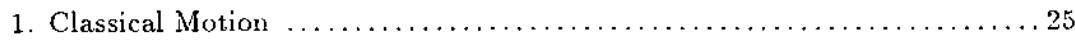

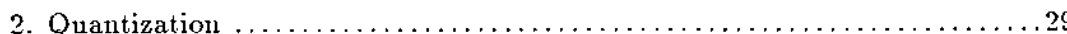

IV. Selberg Supertrace Formula for Super Riemann Surfaces $\ldots \ldots \ldots \ldots \ldots \ldots$

V. Analytic Properties of the Selberg Super Zeta-Functions

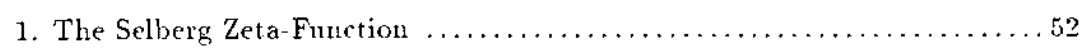

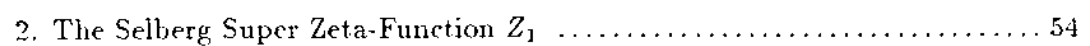

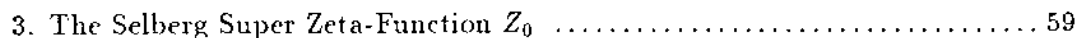

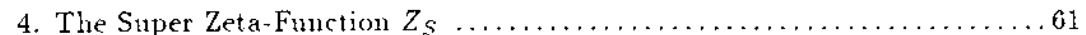

VI. Spectra and Determinants of Laplace-Dirac Operators

1. Resolvent and Heat-Kernel ................................64

2. Discussion of the Spectrum ...............................69

3. Deteminants and the Fermionic String Integrand $\ldots \ldots \ldots \ldots \ldots \ldots$. 1

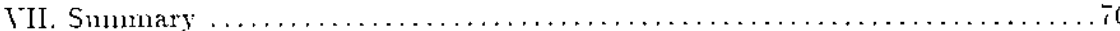

Appendix A: Discusion of Invariance Properties of Iuportant Quantities .......80

Appendix B: Proof of the Path Integral on $S \mathcal{H}$ 83

Appendix (': The Heat-Kernel on SF

Appentix D: The Path Integral on the Prinente lpuer Half-Plane $\mathcal{K} \ldots \ldots \ldots \ldots$ s 


\section{INTRODUCTION}

TOE - Theory Of Everything!

String Theory Includes All Interactions Including Gravitation!

A String Theory Needs Just Two Constants!

There Is An End In Sight For Theoretical Physics!

These are a small selection of the most euphoric claims people have stated for the string theory in the past years (Hawking [48] or Veneziano [90]). But alas! (or fortunately), things are not so easy.

String theory started orginally as a theory, called dual model, for the strong interaction (for reviews see: Allesandrini et al. [1], Schwarz 799], Veneziano [89], Rebb [74], Mandelstam [56] and Scherk [77], compiled by Jacob [52j and Frampton [28]). The idea was based on the observation that many particles lay empirically on straight lines if plotted as mass ${ }^{2}$ over angular momentum - the Regge trajectories. This empirical feature was fitted into a theoretical model first by Veneziano, who introduced the four-point amplitude

$$
A(s, t)=\frac{\Gamma[-\alpha(s)] \Gamma[-\alpha(t)]}{\Gamma[-\alpha(s)-\alpha(t)]}
$$

$\left(\alpha(s)=1+\alpha^{\prime} s\right.$ is the Regge-trajectory, $\alpha^{\prime}$ the "Regge slope", $s, t, u$ Mandelstam variables) and further on by Fubini and Veneziano, who invented characteristic oscillators explaining the Regge behaviour. The name "dual-model" stems from the fact that the four-point amplitude for a scattering process in this model remained independent, whether viewed in the $s, t$ or $u$-channel. Surprisingly, this infinite set of osciliators could be explained by creation and annihilation operators coming directly from a one-dimensional object, called a string, sweeping out a two dimensional surface, called the world sheet, in space-tine. The action (Nambu-Goto action) of this object was simply given by its surface, parametrized by a space-like variable $\sigma$ and a time-like variable $r$ :

$$
S=\frac{1}{2 \pi \alpha^{\prime}} \int_{\tau_{1}}^{\tau_{2}} d \tau \int_{0}^{\pi} d \sigma \sqrt{\left(\frac{\partial X^{\mu}}{\partial \sigma} \cdot \frac{\partial X_{\mu}}{\partial \tau}\right)^{2}-\left(\frac{\partial X^{\mu}}{\partial \sigma}\right)^{2}\left(\frac{\partial X^{\mu}}{\partial \tau}\right)^{2}}
$$

( $X^{\mu}$ embedding in space-time). The typical scale of the string was believed to be the scale of strong interactions: $1 /$ length $\simeq 1 \mathrm{GeV}$. The spectrum which emerged from this Veneziano model revealed a degeneracy at each mass level, asymptotically increasing exponentially with the mass. An important problem was that this spectrum seemed to contain both positive and negative norm states (ghosts), a natural consequence of Lorentz invariance. Thus it was a very strong confirmation of this model that it could be shown in the "No-Ghost-Theorem" that these ghost-states decouple; however, with the strong restriction that the quantized theory only makes sense in 26 dimensions! ${ }^{1}$ A more serious problem remained which was even worse: the appearence of tachyonic states.

Fomulated, e.g. by the action

$$
S=\frac{1}{4 \pi \alpha^{\prime}} \int_{\tau_{1}}^{\tau_{2}} d \tau \int_{0}^{\pi} d \sigma \sqrt{g} g^{n m} \frac{\partial X^{\mu}}{\partial \sigma^{n}} \frac{\partial X_{\mu}}{\partial \sigma^{m}}
$$

${ }^{1}$ Alternatively. it was shown in the light-cone gauge formulation. where only the physical states remain. that the Lorentz algebra cioses only in 26 dimensions. t $g=\operatorname{det}\left(g_{\mathrm{a}_{3}}\right), \sigma_{1}=\tau$ and $\sigma_{2}=\sigma$ denoting the coordinates on the world sheet the (bosonic) string theory reveals a lot of symmetry. The action of Eq.(3) is in variant under arbitrary reparametrisations of the $\sigma_{1} / \sigma_{2}$-surface (diffeomorphisms) under rescalings of the metric (Weyl-transformations) and, naturally, under Lorentz transformations.

To include also strings with fermionic degrees of freedom Ramond i 72 and Neveu and Schwarz 65 introduced the spinning string which possesses even more symmetry if analysed correctly, i.e super Weyl transformations and (rigid or local) supersymmetry (see below). However, the principle problems of the pure bosonic case remain: these were the critical dinjension. which was given for the fermionic string by $D=10$, and the occurrance of taclyons. So the interest in string theory was in general fading away.

Only a few people remained in this subject. In 1974 it was shown by Scherk and Schwarz [ 78 ] that a specific interaction vertex encloses a state which possesses all the ingredients for being a graviton. This opened a quite exciting perspective, namely the view of string theory not as a theory of strong interaction but as a theory which includes gravitation. This also meant that the typical length-scale of a string theory is not of the order of the range of strong interactions (order of the Fermin length $\simeq 10^{-13} \mathrm{~cm}$ ) but of the order of the Planck scale $l_{P l} \simeq 10^{-35} \mathrm{~cm}$ and, respectively the Planck mass $m_{P l}=10^{19} \mathrm{GeV}$. New interest emerged in view of the then developed super symmetry theories and it was quickly shown (Gliozzi, Scherk and Olive i32? GSO-projection) that a specific trunctation of the fermionic string includes a consistent supersymmetric sprectrum. The final breakthrough took place when Green and Schwarz $[35]$ showed that this theory could be formulated in its own terms as a superstring theory and that this theory was anomaly free $[36$ if the gauge group is $S O(32)$ (there are two different types of superstrings, called type I and type II, respectively); this feature is also true for the heterotic string as developed by Gross. Harvey, Martinec and Rohm [44] (with $S O(32)$ and $E_{8} \otimes E_{8}$ as anomaly free gauge groups). Reviews of the superstring theory are due to Green [34] and Schwarz [80;.

Naturally, equivalent but different approaches in string theory were developed: ${ }^{1}$

1) The operator approach: This is the classical canonical approach to string theory and includes a great amount of literature about it (compiled in Green, Schwarz and Witten [37]). Further developments are due to Mandelstam [5i] who calculated trees and loops in a functional integral approach in the light-cone gauge (not to be confuse with the functional integral approach due to Polyakov). In the light-cone gauge only the physical states with positive norm are explicit. However. in this case one must prove the closure of the Lorentz algebra (which gives the critical dimensions $d=26$ and $d=10$ for the bosonic and fermionic string, respectively). In the light-cone gauge the scattering of strings was described by joining and splitting of strings at the endpoints. Much work has been done to get rid of the light-cone formulation which masks any general underlying gauge principle. This lacking gauge principle is one of the problems of all string theories. String theories possess much symmetry, but where does this symmetry come from? The need to compactify the supernumerary dimensions leads - contrary to the hope that the string theory could be unique - to billions upon billions of different theories $\left(\simeq 10^{\mathbf{1 0 0 0}} !\right)$. Recently there has been sone

${ }^{1}$ The idea of two and more dimensional objects like membranes is practically ruled out by the recen paper of de Wit, Lüscher and Nicolai 220 . They showed that the supermembrane has a continuous mass spectrum and no mass gap. 
hope for a classification by means of a fusion algebra developed by E.Verlinde [88]. The aim of a formulation of a complete gauge invariant action for the interacting string could hopefully lead to a deeper understanding of how and why string theory works, and ultimately, what the underlying principle actually is (see e.g. West [93]).

2) The functional (or path integral) approach by Polyakov (see below).

3) BRST quantization by Siegel and Zwiebach [83]. The BRST formalism is a more general procedure for quantization of gauge theories than the Faddeev-Popov approach; not only are more general gauges allowed, but in addition the same BRST transformation which determines the action gives the condition for unitary, as well as determining the gauge-invariant part of the action and the physical states. This is a very appealing feature in view of the difficulties to project from the naive string picture onto the physical states where a "No-Ghost-Theorem" or the Lorentz covariance in the light-cone gauge must be proved. The BRST-invariant action can be written, e.g. simply as [83]:

$$
S=\int L=\int \Phi O Q \Phi,
$$

where $O$ denotes a kinetic operator, $Q$ the BRST-operator and $\Phi$ the string field. Here $\Phi$ includes, of course, so called ghosts. The BRST-operator has the property $Q^{2}=0$, which is an expression for the fact that the BRST-operator determines the physical states, by leaving the Lagrangian $L$ invariant under BRST transformations.

4) There is still another description developed by Witten [94]. This approach removed also the light-cone formalism. In comparison to the interpretation of the interaction of strings in the light cone-gauge one has to perform a rearrangement of strings of equal and fixed lengths, where the rearrangement centers at the nidpoint (for a description see e.g. Jevicki [53]). For three strings: Half of string 1 goes over into 3, while the other baf overlaps with the first half of string ? (in the light-cone gauge string 1 and 2 go over into 3 ). Witten suggested that this feature can be represented by the string field theory Lagrangian

$$
S=\int\left(v^{*} * Q v^{*}+\frac{2}{3} \psi^{*} * \xi^{*} * \psi^{\prime}\right)
$$

where the three-string-overlap corresponds to the three $*$-term (a "wedge-product") and $Q$ is the appropriate BRST operator.

There is also the idea of "p-adic" strings developed by Freund et al.29] and Volovich 922 . This approach is based on the conjecture that at the order of the Planck-length nonlocal properties of strings could be described by p-adic numbers (another completion of the rational numbers $\mathbf{Q}$ based on prime numbers). However, I do not consider this any further.

Let us concentrate on the functional approach of Polyakor $10 \%$. This Ansatz. very simple in its principle but very difficult in explicit calculation. starts in the following way for the closed bosonic string theory. The string pertuluation theory of following way for the closed bosonic string contribution to a scatering amplitude is given by the functional integral over all geometries of a two-dimensional surface of genus $g$ and over the embeddings respectively quant um fields living on this surface. Consider the action of Eq. (3). Then the partition function is given by the functional integral

$$
Z=\int \mathcal{D} g_{n m} \int D X^{\mu^{\prime}-s}
$$

The two-dimensional conformal field theories corresponding to string theories in the critical dimension ( $D=26$ and $D=10$ for the bosonic and fermionic/super-string, respectively) are all free of both local and global anomalies (see, e.g. Friedan [30]) Due to this fact, $g$-loop amplitudes can be reduced to a finite-dimensional integral over the moduli space (Teichmüllerspace) $\mathcal{M}_{g} \cdot{ }^{1}$ More explicitly:

$$
Z=\sum_{g=0}^{\infty} Z_{g},
$$

where

$$
\begin{aligned}
Z_{g} & =\int_{\mathcal{M}_{g}} d(W P)\left[\operatorname{det}^{\prime}\left(\Delta_{0}^{(+)}\right)\right]^{-13} \operatorname{det}^{\prime}\left(\Delta_{1}^{(+)}\right) \\
\Delta_{m}^{( \pm)} & =-y^{2}\left(\frac{\partial^{2}}{\partial x^{2}}+\frac{\partial^{2}}{\partial y^{2}}\right)+i m y \frac{\partial}{\partial x}+m(m \pm 1) .
\end{aligned}
$$

Here the Laplacian $\Delta_{m}^{(+)}$is realised on the Poincaré upper half-plane $\mathcal{H} \equiv\{z=$ $x+i y \mid x \in \mathbf{R}, y>0\}$ (endowed with the hyperbolic geometry), $\mathcal{M}_{g}$ denote the modulispace (Teichmiiller-space) parametrising the variations of all compact surfaces of a fixed gerlus $g$ and $d(W P)$ the Weil-Peterson measure as the integration measure on $M_{g}$. This partition function in the genus $g$, i.e. the multiloop expansion, has been in detail discussed by D'Hoker and Phong [23], Gilbert [31] and Namazie and Rajjev [64]. Especially the determinants in Eq.(8) can be expressed with the theory of the Selberg trace formula on Riemann surfaces $[49,82]$ by means of the Selberg zeta-function. Before explaining this in some detail I want to discuss several realizations of the hyperbolic plane, of which the Poincare upper half-plane is just one. The Poincare upper half-plane is analytically equivalent to three further Riemannian spaces: the pseudosphere $\Lambda^{2}$, the Poincaré disc $D$ and the hyperbolic strip $S$. I start with the 1) pseudosphere $\Lambda^{2}$ which is defined by:

$$
\Lambda^{2}:=\left\{\left(y_{1}, y_{2}, y_{3},-y_{1}^{2}+y_{2}^{2}+y_{3}^{2}=-R^{2}\right\}\right.
$$

(in the following I set $R=1$ ). $A^{2}$ can be visualised as a hyperboloid embedded in a three dimensional Minkowski space. But be careful: $\Lambda^{2}$ has negative Gaussian curvature $K=-1$, as well as $\mathcal{H}, D$ and $S$, i.e.they are everywhere saddle-shaped A more convenient description for $A^{2}$ reads in pseudospherical polar coordinates $(\tau . \delta): 8,85,91$ i:

$$
y_{1}=\cosh \tau, \quad y_{2}=\sinh \tau \cos \phi . \quad y_{3}=\sinh \tau \sin \phi, \quad(\tau-0 . \phi \epsilon[0.2 \pi) .
$$

The metric $g_{a b}$ associated with the line element $d s^{2}=g_{a b} d q^{a} d q^{b}$ reads $g_{a b}=\operatorname{diag}$ (1. $\sinh ^{2} T$ ).

2) With the stereographic projection of $\mathrm{A}^{2}$ onto the $\left(x_{1}, x_{2}\right)$-plane I get the Poincaré $\operatorname{disc} D-\left\{z=x_{1}+i x_{2} x_{1}^{2} \div x_{2}^{2} \cdot 1\right\}$ :

$$
=-\cdots x_{1}+i x_{2}=r \epsilon^{i \omega}=\frac{y_{2}-i y_{3}}{1+y_{1}}=\tanh \frac{\tau}{2}\left(\sin Q+i \cos \phi^{\prime}\right) .
$$

Belavin and Knizlmik i12: bave shown that the string amplitudes are essentially the product of an analytic and an anti-analyitic function on $\mathcal{M}_{y}$. This factorization tas a direct correspondence with analytic and an anti-analyitic function on $\mathcal{M}_{y}$. This factorization
the decomposition of the string modes into lefi and right movers.

"In the case of the bosonic string this imlegrand can also be expressed by Theta-finctions 2.58 A super analogue of the Theta-function does not vet exist. But see. however. Mlanin 58 , for a conjecture. 
Figure 1: The fundamental domains $(g=2)$ in the three Riemannian spaces $\mathcal{H} . D . S$
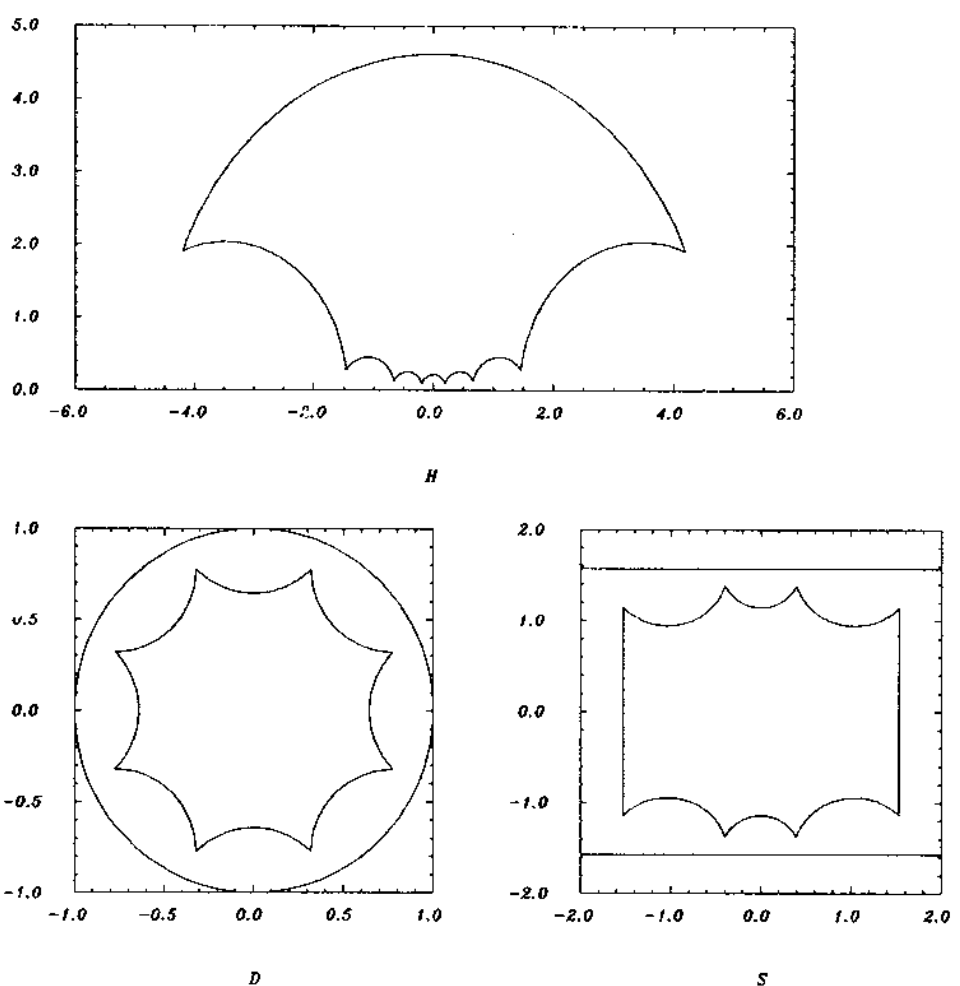

Here the metric reads $\left.g_{a b}=2 /\left(1-r^{2}\right)\right]^{2} \operatorname{diag}\left(1, r^{2}\right)$.

3) The Poincaré disc $D$ can be mapped onto the Poincare upper half-plane $\mathcal{H}$ by the Cayley-transformation:

$$
\zeta=x+i y=\frac{-i z+i}{z+1}, \quad z=\frac{-\zeta+i}{\zeta+i} .
$$

The metric reads $g_{a b}=1 / y^{2} \cdot \delta_{a b}$.

4) With the help of the transformation

$$
\eta=X+i Y=-\ln (-i \zeta)(=2 \operatorname{artanh} z)
$$

one can map the Poincare upper half-plane (the Poincare disc) onto the hyperbolic strip $S=\left\{\eta=X+i Y|X \in \mathbf{R}| Y:,<\frac{\pi}{2}\right\}$. The metric reads $g_{a b}=1 / \cos ^{2} Y \cdot \delta_{a b}$. The hyperbolic distance $r=d\left(p^{\prime \prime}, p^{\prime}\right)\left[p\right.$ - any of the coordinates $(\tau, \delta),\left(x_{1}, x_{2}\right),(x, y)$,
$\left(\mathrm{X}^{-}\right)^{-}$in these spaces is given by:

$$
\begin{aligned}
& \left.\cosh r=\cosh \tau^{\prime \prime} \cosh \tau^{\prime} \quad \sinh \tau^{\prime \prime} \sinh \tau^{\prime} \cos \left(\phi^{\prime \prime}-\phi^{\prime}\right) \quad \text { (on } \Lambda^{2}\right) \\
& \because 1+\frac{2 z^{\prime \prime}-z^{\prime 2}}{\left(1-z^{2}\right)\left(1-z^{\prime \prime}\right)} \\
& =\frac{\left(r^{\prime \prime}-x^{t}\right)^{2}-y^{n z}-y^{\prime 2}}{2 y^{\prime} y^{\prime \prime}}
\end{aligned}
$$

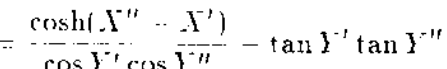

Figure 2: The fundamental domains of Fig.1 boosted once in all eight directions in the three Riemannian spaces $\mathcal{H}, D$ and $S$
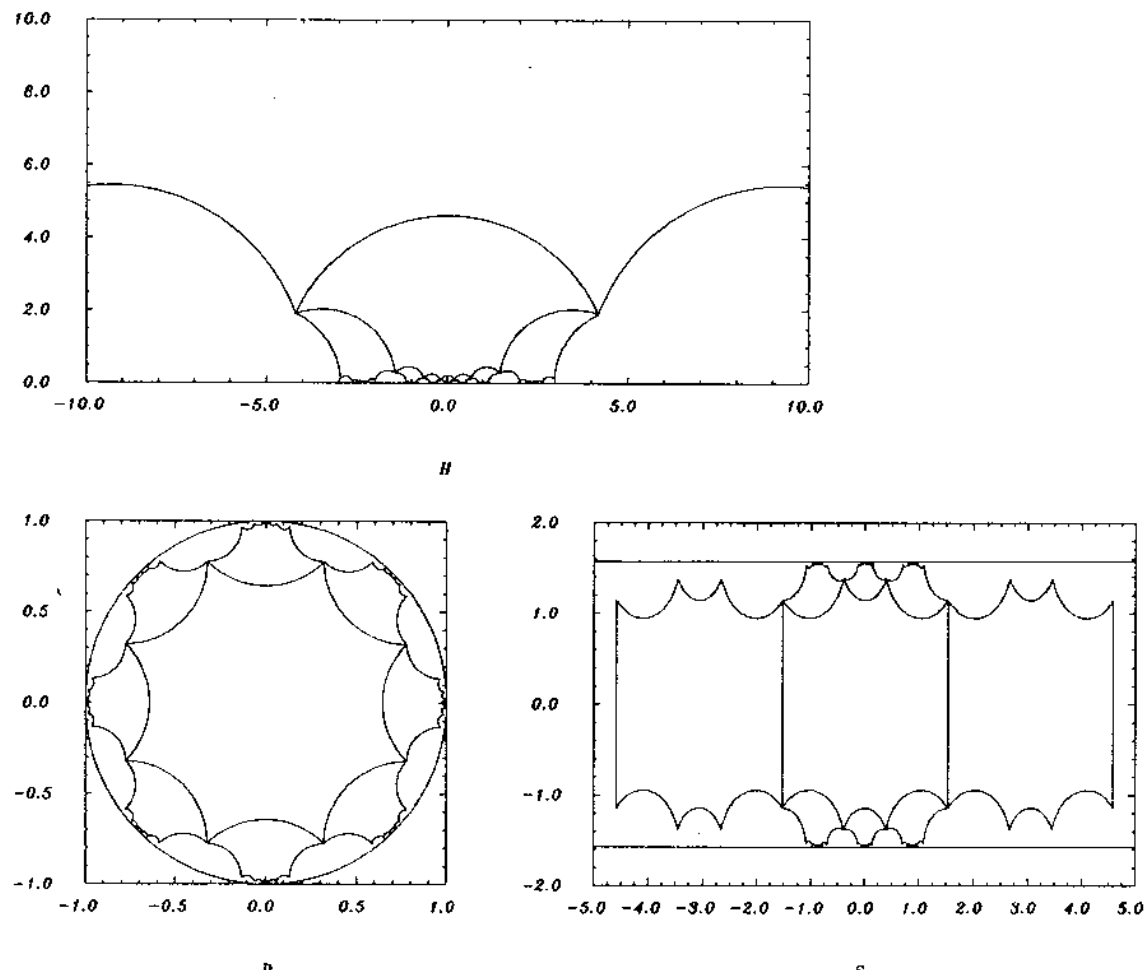

The generators of the symmetrical fundamental domain for a compact Riemanu surface of general genus $g$ read in $\mathcal{H}$ :

$\gamma_{k}=\left(\begin{array}{cc}\cosh \frac{l_{0}}{2}-\sinh \frac{l_{0}}{2} \cos \frac{k \pi}{2 g} & \sinh \frac{l_{0}}{2} \sin \frac{k \pi}{2 g} \\ \sinh \frac{l_{0}}{2} \sin \frac{k \pi}{2 g} & \cosh \frac{l_{0}}{2}+\sinh \frac{l_{0}}{2} \cos \frac{k \pi}{2 g}\end{array}\right) . \quad(k=0, \ldots, 2 g-1)$, 
including the inverse generators $\gamma_{k}^{-1}=\left(\gamma_{k}\right)^{-1}$ and where $\cosh \frac{l_{0}}{2}=\cot \frac{\pi}{4 g}$. In particular for $g=2$ :

$$
\gamma_{k}=\left(\begin{array}{cc}
\cosh \frac{l_{0}}{2}-\sinh \frac{l_{0}}{2} \cos \frac{k \pi}{4} & \sinh \frac{l_{0}}{2} \sin \frac{k \pi}{4} \\
\sinh \frac{l_{0}}{2} \sin \frac{k \pi}{4} & \cosh \frac{l_{0}}{2}+\sinh \frac{l_{0}}{2} \cos \frac{k \pi}{4}
\end{array}\right),
$$

and $\cosh \frac{l_{0}}{2}=\cot \frac{\pi}{8}=1+\sqrt{2}=2.41421 \ldots$ These generators are also called boosts, because they correspond to explicit Lorentz transformation on the pseudosphere $\Lambda^{2}$. (For their action on elements $\zeta \in \mathcal{H}$ see Eq.(19) below.) They obey the important constraint

$$
\left(\gamma_{0} \gamma_{1}^{-1} \ldots \gamma_{2 g-2} \gamma_{2 g-1}^{-1}\right)\left(\gamma_{0}^{-1} \gamma_{1} \ldots \gamma_{2 g-2}^{-1} \gamma_{2 g-1}\right)=\left(\begin{array}{ll}
1 & 0 \\
0 & 1
\end{array}\right)=\mathbf{1}_{2} .
$$

In figure 1 I have displayed the fundamental domain for the simplest case of genus $g=2$ and a symmetrical fundamental domain. The various parts of the figure denote the fundamental domain if mapped onto $D, S$ and $\mathcal{H}$, respectively. Figure 2 displays the action whereby the generators transform the fundamental domain in $\mathcal{H}, S$ and $D$, respectively. Note that in $\mathcal{H}$ the boost in the positive $y$-direction produces such a large boosted domain that it is not included in the figure (in fact, a ten-times larger box would be needed).

As it is known from the theory of Riemann surfaces and automorphic functions, every compact Riemann surface of genus $g \geq 2$ can be mapped onto the Poincaré upper half-plane $\mathcal{H}=\{z=x+i y \mid y>0\}$ (endowed with the hyperbolic geometry) into a polygon with $4 g$ edges. This polygon $P$ can be associated with a Fuchsian group $\Gamma$ which tesselates the Poincaré upper half-plane in an unique way (see e.g. Fenn $[26]$ and the above figures for $g=2$ ). A specific polygon $P$ can mapped onto another one $p^{\prime}$ by an element $\gamma \in \Gamma$ (boosts). The property of this group $\Gamma$ is that it is strictly hyperbolic (i.e. the trace of its elements is larger than two). On the Poincaré upper half-plane $\Gamma$ is realized in terms of discrete subgroups of the group $P S L(2, \mathbf{R})$ [on the disc $D$ in terms of discrete subgroups of $S U(1,1)$ ], i.e. the special linear projections, written in matrix notation as

$$
\gamma=\left(\begin{array}{ll}
a & b \\
c & d
\end{array}\right)
$$

with $a, b, c, d \in \mathbf{R}, a d-b c=1$ and $\operatorname{tr}(\gamma)>2$. Its action on $\mathcal{H}$ reads

$$
\gamma \zeta=\zeta^{\prime}=\frac{a \zeta+b}{c \zeta+d}
$$

Further I consider closed geodesics in the polygon $P$ which represents a fundarnental domain of $\Gamma$. I denote these geodesics by $l$, where there is a direct relation between a geodesic $l$ and an element,$\in \Gamma$ by $2 \cosh \frac{l_{2}}{2}=|a+d|$. For the length spectrum of $P$ one has Huber's law $\#(x)=\epsilon^{x} / x(x \rightarrow \infty)$, where $\#(x)$ is the number of inconjugate primitive $\gamma$ 's with $l_{\gamma} \leq x$. Here, elements,$\in \Gamma$ which are not powers (greater or equal to 2) of any element in $\Gamma$ are called primitive elements of $\Gamma$.

Consider the Laplace operator $\Delta_{0}^{(-)}=-\Delta--y^{2}\left(\partial_{x}^{2}-\partial_{y}^{2}\right)$ and a function $h(p)$ which satisties the following conditions i) $h(p)$ is an even function in $p$,

ii) $h(p) \propto\left(1+|p|^{2}\right)^{-1+\epsilon}(p \rightarrow \pm \infty, \epsilon>0), \epsilon>0$.

iii) $h(p)$ is holomorph in the strip $|\operatorname{Im}(p)| \leq \frac{1}{2}+\epsilon, \epsilon>0$.

Denote by $g(u)$ the Fourier transformed of $h(p)$ :

$$
g(u)=\frac{1}{2 \pi} \int_{-\infty}^{\infty} h(p) e^{-i u p} d p .
$$

Now consider this Laplacian (which is invariant under the action of $\Gamma$ ) in a fundamental domain of $\Gamma$ and the Eigenvalue problem

$$
-\Delta \Psi(z)=E \Psi(z)
$$

with periodic boundary conditions $\Psi(\gamma z)=\Psi(z),(\gamma \in \Gamma)$. Parametrize the Eigenvalues $E_{n}$ by $E_{n}=s_{n}\left(1-s_{n}\right)$ with $s_{n}=\frac{1}{2}+i p_{n}$. Then the Selberg trace formula reads

$$
\sum_{n=0}^{\infty} h\left(p_{n}\right)=2(g-1) \int_{-\infty}^{\infty} p \tanh \pi p h(p) d p+\sum_{\{\gamma\}_{p}} \sum_{k=1}^{\infty} \frac{l_{\gamma}}{\sinh \frac{k l_{\gamma}}{2}} g\left(k l_{\gamma}\right)
$$

expressing an exact relation between the (quantum mechanical) trace of the operator valued function $\tilde{h}(-\Delta)$ with $\bar{h}\left(p^{2}+\frac{1}{4}\right)=h(p)$ and the (classical) length spectrum $\left\{l_{\gamma}\right\}$ [49]. On the left hand side of Eq.(22) the sum runs over the pairs $p_{n},-p_{n}$ (corresponding to the same Eigenvalue $E_{n}$ ), $p=0$ has to be counted twice if happens to be an Eigenvalue. On the right hand side the sum is taken over all primitive conjugacy classes in $\Gamma$ denoted by $\{\gamma\}_{p}$. For the case of genus $g=2$ and the symmetrical fundamental domain of $E_{q} .(16)$ the lower part of the length spectrum was first calculated by Aurich and Steiner [5] coming in the end up to 200 million lengths $[6]$ (including multiplicities). In a very beautiful paper by Aurich, Sieber and Steiner $[\boldsymbol{r}]$ Eq.(22) was used to calculate the energy levels $E_{n}$ by an appropriate test function $h$-and vice versa by first solving the Eigenvalue problem and then calculate the first primitive lengths.

Note: The condition iii) on the test function $h$ sets a growth condition on the Fourier transform $g$, which ensures the absolute convergence of all sums in question (note Huber's law).

Now introduce the Selberg zeta-function as

$$
Z(s):=\prod_{\{\eta\}_{p}} \prod_{n=0}^{\infty}\left[1-\epsilon^{-\{s+n) l_{\gamma}}\right], \quad(\operatorname{Re}(s) \cdots 1) .
$$

Huber's law assures the convergence of $\mathrm{E}_{4}(23)$ if $\operatorname{Re}(s)>1$. Again the product runs over all primitive inconjugate,$\in \Gamma$. With the help of Eqs.(22) and (23) one call calculate determinants of the Laplacians $\Delta_{m}^{(+)}$, where e.g. $\{16,84\}$ :

$$
\begin{aligned}
\operatorname{det}^{\prime}(-\Delta) & =Z^{\prime}(1) c^{\left.(g-1) ! \ln 2 \pi+4 c^{\prime}(-1)-\frac{1}{2}\right)} . \\
\operatorname{det}^{\prime}\left(\Delta_{1}^{(+)}\right) & =Z(2) \epsilon^{(g-1) \mid 3 \ln 2 \pi+4 c^{\prime}(-1) \cdot \frac{e}{2}}
\end{aligned}
$$

(the prime on the determinants denote the omission of zero modes). For the calcu. lation of $\operatorname{det}\left(\Delta_{m}^{t-1}\right)$ one has to use the $m$-generalisation of the trace formulat, i.te the 
Selberg trace formula for automorphic forms of weight $m$ [49]. Under the same conditions on the test function $h$ one gets (assuming that no elliptic elements appear in the group $\Gamma$ ) the Selberg trace formula (49):

$$
\sum_{n=0}^{\infty} h\left(p_{n}\right)=2(1-g) \int_{0}^{\infty} \frac{g^{\prime}(u)}{\sinh \frac{u}{2}} T_{m}\left(\cosh \frac{u}{2}\right) d u+\sum_{\{\gamma\}_{p}} \sum_{k=1}^{\infty} \frac{l_{\gamma}}{\sinh \frac{k l_{\gamma}}{2}} g\left(k l_{\gamma}\right),
$$

where $T_{m}\left(\cosh \frac{u}{2}\right)=\cosh \frac{m}{2} u$ denotes the $m^{t h}$ Chebyshev-polynomial in $\cosh \frac{u}{2}$. For $m=0$ one recovers Eq.(22). Here the Eigenvalues $E_{n}$ (parametrized by $E_{n}=\frac{1}{4}+p_{n}^{2}$ ) are the Eigenvalues of the Laplacian ${ }^{1}-\Delta_{m}=-\Delta+i m y \partial_{x}$. The boundary conditions on the Eigenstates $\Psi_{n}$ have to be appropriatiy changed according to $\Psi(\gamma z)=$ $\chi_{\gamma}{ }^{m}(c z+d)^{m}[|c z+d|\}^{-m} \Psi(z)(\gamma \in \Gamma)$, where $\chi_{\gamma}$ is a possible additive character.

A procedure similar to that used for the bosonic string can be used for the fermionic string where the relevant action reads $[17,19,50]$ :

$$
\begin{aligned}
S(g, X, \chi, \psi)=\frac{1}{4} \int_{M} d^{2} \sigma \sqrt{g} & {\left[\frac{1}{2} g^{m n} \partial_{m} X^{\mu} \partial_{n} X^{\mu}+i \bar{\psi}^{\mu} \gamma^{m} \partial_{m} \psi_{\mu}\right.} \\
& \left.-F^{\mu} F_{\mu}-\bar{\chi}_{a} \gamma^{m} \gamma^{a} \psi^{\mu} \partial_{m} X_{\mu}+\frac{1}{8} \bar{\psi}^{\mu} \dot{\psi}_{\mu} \bar{\chi}_{a} \gamma^{a} \gamma^{b} \chi_{b}\right] .
\end{aligned}
$$

Here denote:

1) $M:$ the two-dimensional world sheet,

2) $\mathcal{D} X^{\mu}$ : imbeddings in space-time $(D=10)$,

3) $g_{m n}=\epsilon_{m}{ }^{a} e_{n}{ }^{b} \delta_{a b}$ : metric on the world sheet,

4) $\psi^{\mu}$ : real (Majorana-) spinor,

5) $\chi_{a}:$ spin $\frac{3}{2}$-gravitino field

6) $F^{\mu}$ : nondynamical field which is needed to close the supersymmetric algebra off shell $[51,77]$ :

$$
\delta X=i \bar{\epsilon} \psi^{4}, \quad \delta \Psi=\partial_{a} X \gamma^{a} \epsilon+F \epsilon, \quad \delta F=i \bar{\epsilon} \gamma^{a} \partial_{a} \psi,
$$

where $\epsilon$ is a two-dimensional spinor. One sets $F=0$, since the equation of motion just reads $F=0$

7) $\gamma^{a}(a=0,1,5)$ denote the $\gamma$-matrices

$$
\gamma^{0}=\left(\begin{array}{cc}
0 & 1 \\
-1 & 0
\end{array}\right), \quad \gamma^{1}=\left(\begin{array}{cc}
0 & 1 \\
1 & 0
\end{array}\right), \quad \gamma^{5}=\left(\begin{array}{cc}
1 & 0 \\
0 & -1
\end{array}\right),
$$

and a bar over quantities denotes complex conjugation. The action $(26)$ is invariant under five fundamental symmetries [24]

i) Reparametrisation invariance,

$$
\begin{aligned}
\delta \epsilon_{m}{ }^{a} & =\delta V^{n} \partial_{n} \epsilon_{m}{ }^{a}+\epsilon_{n}{ }^{a} \partial_{n} \delta V^{n} \\
\delta \chi_{m} & =\delta V^{n} \partial_{n, \chi_{m}}-\chi_{n} \partial_{m} \delta V^{n} \\
\delta X^{\mu} & =\delta V^{n} \partial_{n} X^{\mu} \\
\delta \Psi^{\mu} & =\delta V^{n} \partial_{n} \Psi^{\mu} .
\end{aligned}
$$

ii) Supersymmetry transformations,

$$
\begin{aligned}
& \delta \epsilon_{m}{ }^{a}=i \xi \gamma^{a} \chi_{m}, \quad \delta \chi_{m}=2 D_{m} \xi, \quad \delta X^{\mu}=\xi \Psi^{\mu}, \\
& \delta \Psi_{\mathrm{a}}^{\mu}=-\frac{1}{2} i\left(\gamma^{n}\right)_{a}{ }^{\beta} \xi_{\beta}\left(\chi_{n} \Psi^{\mu}\right)+i\left(\eta^{m}\right)_{0}{ }^{\beta} \xi_{\beta} \partial_{m} X^{\mu} .
\end{aligned}
$$

Here $D_{m}=\partial_{m}-\frac{1}{2} \omega_{m} \gamma^{5}$ are covariant derivatives taken with the connection $\omega_{n z}:=\epsilon_{m}{ }^{a} \epsilon^{p q} \partial_{p} c_{q}{ }^{b} \delta_{a b}-\frac{i}{2} \chi_{m} \gamma_{5} \gamma^{n} \chi_{n}$, where $\epsilon_{a b}$ is the totally antisymmetric tensor for the raising and lowering of spinor indices.

iii) Weyl transformations,

$$
\begin{aligned}
\delta \epsilon_{m}{ }^{a} & =\Lambda \epsilon_{m}{ }^{a}, & & \delta \Psi^{\mu}=-\frac{1}{2} \Lambda \Psi^{\mu}, \\
\delta \chi_{\eta_{2}} n & =\frac{1}{2} \Lambda_{\chi_{m}}, & & \delta X^{\mu}=0 .
\end{aligned}
$$

iv) super-Weyl transformations

$$
\delta_{\lambda_{m}}=\gamma_{m} \lambda, \quad \delta(\text { anything else })=0 .
$$

v) Local Lorentz transformations,

$$
\begin{aligned}
\delta e_{m}{ }^{a} & =l \epsilon^{a b} e_{m b}, & & \delta \Psi^{\mu}=\frac{1}{2} l \gamma_{5} \Psi^{\mu}, \\
\delta \chi_{m} & =\frac{1}{2} l \gamma_{5} \chi_{m}, & \delta X^{\mu} & =0 .
\end{aligned}
$$

(With $\delta V^{n}$ an infinitesimal vector field, $\xi$ an infinitesimal spinor, $\Lambda$ and $\lambda$ an infinitesimal scaling-function and $l$ an infinitesimal Lorentz transformation, respectively.)

It is very important that this action can be cast into a compact form if a superspace notion is used $[50]$. Consider a $(2+2)$-dimensional superspace with coordinates $Z^{M}:=\left(x^{\mu}, \theta^{m}\right)$ with commuting $x^{\mu}$ and anticommuting $\theta^{m}$ (for more details see $[50]$ and chapter II). Introduce the Vierbein $E_{M}{ }^{A}$ :

$$
\begin{array}{ll}
E_{\mu \mu}{ }^{a}=\epsilon_{\mu}{ }^{a}+i \theta^{\dagger} \gamma^{0} \gamma^{a} \chi_{\mu \mu} & E_{m}{ }^{a}=i\left(\theta^{\dagger} \gamma^{0} \gamma^{\alpha}\right)_{m} \\
E_{\mu}{ }^{a}=\frac{1}{2}\left[\lambda_{\mu}{ }^{a}-\omega_{\mu}\left(\gamma_{5} \theta\right)^{a}\right] & E_{m}{ }^{a}=\delta_{m}{ }^{a} .
\end{array}
$$

Here, $\epsilon_{\mu}{ }^{\alpha}$ is the $x$-space Vierbein and $e=\operatorname{det}\left(\epsilon_{\mu}{ }^{\alpha}\right)$. Now define

$$
\Phi(Z)=X(x)+i \theta^{\dagger} \gamma^{0} \psi(x)+\frac{i}{2} \theta^{\dagger} \gamma^{0} \theta F(x)
$$

Then the action (26) can be rewritten as

$$
\begin{aligned}
& S=\frac{1}{4} \int d x^{1} d x^{2} d \theta_{1} d \theta_{2} \epsilon L \\
& L=E_{\mathrm{a}}{ }^{M} \partial_{m} \Phi(Z) E^{a N} \partial_{N} \Phi(Z)
\end{aligned}
$$

The equations of motion read

$$
D_{\mathrm{a}} \ddot{D}^{\alpha} \Phi(Z)=0 \text {. }
$$


where $D_{\alpha}=E_{\alpha}{ }^{M} \partial_{m}$. Mapping (in the sense that I want to study partition functions) a closed compact world sheet onto a fundamental domain (of a super Fuchsian group) on the Poincare super upper half-plane $S \mathcal{H}$ I get the Laplace-Dirac operator $\square_{0}=$ $2 Y D \bar{D}$ which $I$ have to study $\left(Y=\operatorname{Im}(z)+\frac{\theta \bar{\theta}}{2}=y+\theta \bar{\theta} / 2, \theta=\theta_{1}+i \theta_{2}, \bar{\theta}=\theta_{1}-i \theta_{2}\right)$. The action $S$ rewritten in terms of $\square$ o reads

$$
S=-\frac{1}{4} \int d V(Z) \Phi(Z) \square_{0} \Phi(Z),
$$

where $d V(Z)=d z d \bar{z} d \theta d \bar{\theta} / 2 Y$ is the invariant measure on $S \mathcal{H}$. The partition function is calculated as follows:

$$
\begin{aligned}
Z & =\sum_{g=0}^{\infty} Z_{g} \\
Z_{g} & =\int \mathcal{D} g_{a b} \int \mathcal{D} \chi_{a} \int \mathcal{D} X^{\mu} \int \mathcal{D} \psi^{\mu} e^{-S\left(g_{1} X, \chi, \psi\right)} .
\end{aligned}
$$

Analogous considerations as in the bosonic case yield [9]:

$$
Z_{g}=\int_{S \mathcal{M}_{g}} d(S W P)\left[\operatorname{sdet}\left(-\square_{b}^{2}\right)\right]^{-\frac{5}{2}}\left[\operatorname{sdet}\left(-\square_{2}^{2}\right)\right]^{\frac{1}{2}},
$$

where (in the notation I am using):

1) $S \mathcal{M}_{g}:$ super moduli space,

2) $d(S W P)$ : super Weil-Peterson measure,

3) $\square_{m}=2 Y D \bar{D}-m(i \theta-\bar{\theta}) \bar{D}$,

In the fermionic string, the spinors on the Riemann surface are defined with some spinor structure, which can be independently choosen for left- and right novers. In type II superstring theory ${ }^{1}$, these spinor structures must be summed over to project (GSO-projection) onto the correct sector of the Neveu-Schwarz-Ramond theory [24]. It tur partition function vanishes by the use of a famous Jacobi identity on theta-functions partition function vanishes by the use of a famous Jacobi identity on theta-functions
("aequatio identica satis abstrusa"), indicating the presence of 10-dimensional space("aequatio identica satis abstrusa"), indicating the presence of 10-dimensional space and fermionic states (for details see e.g. [24]).

Thro dimension $d=10$

The contents of the chapters will be as follows:

The second chapter will deal with the theory of Grassmann numbers, superanalysis and super Riemann surfaces. Grassmann numbers or odd numbers are introduced, respectively, in an elementary way; supernumbers as combinations of ordinary numbers and Grassmann numbers are defined; furthermore, super analytic functions a functions of super variables with super coefficients are defined. Differentiation and integration are introduced which are very simple for Grassmann numbers because functions of Grassmann numbers can be at most linear due to their nilpotent property. Supervector spaces, supertraces and superdeterminants are considered. Having

'Type I theories contain open strings, whereas type II theories only closed strings. this machinery it is an easy task to proceed to super manifolds and super Riemann surfaces. The main application will be to consider and derive the explicit form of superconformal automorphisms on super Riemann surfaces, which includes the statement of an super uniformisation theorem.

The third chapter is devoted to the study of physics on the super Poincare upper half-plane $S \mathcal{H}$, which is a special super Riemann surface and can be tesselated by a generalisation of $S L(2, \mathbf{R})$ into compact super Riemann surfaces whith fixed genus $g$ Classical and quantum physics will be considered which will give a Lagrangian and Hamiltonian formalism. Furthermore the important Laplacians and Dirac operators on $S \mathcal{H}$ are introduced, which take on an important role in the Polyakov approach to the fermionic string theory. Also I note the path integral approach on $S \mathcal{H}$.

With the fourth chapter starts the main part of my paper. I discuss and derive the Selberg supertrace formula for automorphic forms of weight $m$ on compact super Riemann surfaces, which are visualized as bounded domains on the super Poincaré upper half-plane $S \mathcal{H}$. This trace formula was already derived by Baranov et al. [10], but in their discussion the term corresponding to the unit transformation (except $m=0$ ) was missing. This term is explicitly derived and thus their work completed. However, I do not claim to be mathematically rigorous.

The fifth chapter is devoted to the discussion of the two Selberg super zeta-functions and contains entirely new results. Zeta-functions were originally introduced by Selberg [82] in order to study spectra of Laplacians on compact Riemann surfaces of genus $g$. The super Selberg zeta-functions are similarly defined as the usual Selberg zetafunction. I find similarities but also important differences for $Z_{0}$ and $Z_{1}$ in comparison with the usual Selberg zeta-function. I derive functional relations for $Z_{0}, Z_{1}$ and a relation linking these two functions.

In the sixth chapter I apply my results to the fermionic string theory. This includes first the discussion of the spectra of the Laplace-Dirac operators. and second the calculation of their determinants. It is shown that the relevant determinants which have to be considered in the Polyakov functional integral exist and are finite. Discussions of the superdeterminants are due to Baranov et al. $[10]$ and Aoki $[3]$. In Ref.110 ratios of superdeterminants corresponding to different copies of super Fuchsian groups were considered (due to lack of knowledge of the analytic behaviour of super zeta-functions). In Ref. 3 ! attempts have been made to express the superdeterminants by the super zeta-functions, where the functional equation for the usual Selberg zeta-function has been used, which is, however, questionable. Furthermore the behaviour of the superdeterminants of the operators $\square_{m}$ in the case of degenerate super Riemann surfaces is discussed.

Chapter VII contains a summary and concluding remarks.

In Appendix A the invariance properties.. of some important quantities is discussed. In Appendix $B$ the validity of the path integral formulation on $S \mathcal{H}$ is shown and in Appendix $C$ some results of Aoki are summarized, who calculated the heat-kernel on $S \mathcal{H}$. Appendix D deals with the result of path integration on the Poincare upper-half plane $\mathcal{H}$. 


\section{SUPER Riemann Surfaces and Super Uniformisation}

\section{Survey of Superanalysis}

In this chapter I want to give a short survey of superanalysis and supermanifolds to make the material available for the next sections. No new results are presented and I do not claim completeness. Most of this section was acquired in collaboration with Holger Ninnemann and already presented in a seminar talk in the summersemester 1988 at the University of Hamburg [66]. The text is based on DeWitt [22], Rabin and Crane [71] and Rogers [76]. A compiled version of superanalysis can be found in the book of Berezin [14].

Let us start with the essentials.

Def.1: Let $\Lambda_{N}$ be the Grassmann-algebra over $\mathbf{C}$ which is generated by the elements $\zeta_{a}(a=1, \ldots, N) . \Lambda_{N}$ forms a $2^{N}$-dimensional vector space with basis

$$
1, \zeta^{a}, \zeta^{a} \zeta^{b}, \ldots \quad(a<b)
$$

and the anticommutation relation

$$
\zeta^{a} \zeta^{b}=-\zeta^{b} \zeta^{a} \quad \forall a, b .
$$

Often one considers the formal limit $N \rightarrow \infty$ and the infinite dimensional vector space $\Lambda_{\infty}$. Following Rogers one should not be concerned about introducing as much of elements $\zeta^{a}$ as one needs: "To those physicists who use supermanifolds, but do not often lie awake at night worrying about the finer points of analysis, the message of this paper is simple - if you need more generators for your Grassmann algebra, help yourself!"

The anticommutation relation (2) gives at once for $a=b:\left(\zeta^{a}\right)^{2}=0$. Anticommuting variables were first introduced by Schwinger [81; Martin [59] and Berezin [13]. However, really concluding $\zeta^{2}=0$ is due to the latter two authors.

Def.2: The elements of $z \in \Lambda_{\infty}$ are called supernumbers and can be decomposed as

$$
\begin{gathered}
z=z_{B}+z_{S}, \quad z_{B} \in \mathbf{C} \\
z S=\sum_{n=1}^{\infty} \frac{1}{n !} c_{a_{1} \ldots \ldots a_{n}} \zeta^{a_{n}} \ldots \zeta^{a_{1}}
\end{gathered}
$$

with $c_{a_{1}, \ldots, a_{n}} \in \mathbf{C}$ totaly antisymmetric. $z_{B}$ and $z_{S}$ are called the body and the soul of the supernumber $z$, respectively.

A supernumber has an inverse only iff $z_{B} \neq 0$. It is given by

$$
z^{-1}=z_{B}^{-1} \sum_{n=0}^{\infty}\left(-z_{B}^{-1} z_{S}\right)^{n} .
$$

Every supernumber $z \in \Lambda_{\infty}$ can be decomposed into an even and odd contribution:

$$
\begin{aligned}
& z=u+v \\
& u:=u_{B}+u_{S}=z_{B}+\sum_{n=1}^{\infty} \frac{1}{(2 n) !} c_{a_{1} \ldots, a_{2 n}} \zeta^{a_{2 n}} \ldots \zeta^{a_{1}} \\
& v:=\sum_{n=0}^{\infty} \frac{1}{(2 n+1) !} c_{a_{1}, \ldots, a_{2 n+1}} \zeta^{a_{2 n+1}} \ldots \zeta^{a_{1}} .
\end{aligned}
$$

The number $r$ is pure soul and can not be inverted. Furthermore $r^{2}=0$

Def.3: The even supernumbers $u$ are called (type-) c-numbers and commute with all other numbers. The set of ail c-numbers is denoted by $\mathbf{C}_{c}$

The odd supernumbers are called (type-) a-numbers and the set of ail a-numbers is denoted by $\mathbf{C}_{a}$.

Let us pass to superanalytic functions

Def.4: Let $z=u+v\left(u \in \mathbf{C}_{c}, v \in \mathbf{C}_{a}\right), f: \Lambda_{\infty} \rightarrow \Lambda_{\infty}$. The function $f$ is called superanalytic if:

(i) $f$ is a linear function in the odd argument

$$
f(z)=g_{1}(u)+v g_{2}(u)
$$

(ii) there exist analytical functions $h_{k}: \mathbf{C} \rightarrow \Lambda_{\infty} \quad(k=1,2)$, so that the functions $g_{k}: \mathbf{C}_{c} \rightarrow \Lambda_{\infty} \quad(k=1,2)$ can be written as a power series expansion in $u_{S}$

$$
g_{k}(u)=\left.\sum_{n=0}^{\infty} \frac{1}{n !} \frac{d^{n} h_{k}}{d x^{n}}\right|_{x=u_{B}} u_{S}^{n} .
$$

The condition (i) is a natural generalisation of the definition of an "ordinary" analytic function. The power series expansion in the odd arguments can be at most linear and a superanalytic function $f: \mathbf{C}_{a} \rightarrow \Lambda_{\infty}$ is superanalytic on whole $\mathbf{C}_{a}$

Def.5: Differentiation with respect to the c-coordinate is the same as for ordinary functions over $\mathbf{C}$. Differentiation with respect to the a-coordinates has to be done by commuting or anticommuting the a-variable to the differential operator. Example (differentiation from the left):

$$
\frac{d}{d v}(a+v b)=b=b_{c}+b_{a}
$$

but

$$
\frac{d}{d v}(a+b v)=\frac{d}{d v}\left[a+v\left(b_{c}-b_{a}\right)\right]=b_{c}-b_{a}
$$

$\left(a, b \in \Lambda_{\infty}, b_{c} \in \mathbf{C}_{c}, b_{a} \in \mathbf{C}_{a}\right)$

Def.6: Integration over $\mathbf{R}_{a}$ is defined by

$$
\int_{\mathbf{R}_{a}} d x=0, \quad \int_{\mathbf{R}_{a}} d x x=1=-\int_{\mathbf{R}_{a}} x d x .
$$

Integration and differentiation with respect to Grassmann variables is a formal procedure and was introduced by Berezin [13j. " $d x$ " does not describe any infinitesimal. In the following the index $\mathbf{R}_{a}$ in the Grassmann integration will be ondtted.

A comparison of Eqs.(8) and (9) yields that differentiation and integration with re spect to Grassmann variables are identical. Let $f(x)=a+x b$ with $a, b \in \Lambda_{\propto}, x \in \mathbf{R}_{a}$ Then

$$
\begin{aligned}
\frac{d}{d x} f(x) & =b \\
\int d x f(x) & =b .
\end{aligned}
$$


This curious result shows again that differentiation and integration are only formal procedures.

Def.7: Let $f$ as before in Eq.(10). The $\delta$-function on $\mathbf{R}_{a}$ is defined by

$$
\begin{aligned}
\delta(x) & =x, \quad x \in \mathbf{R}_{a} \\
\int d x \delta(x) f(x) & =\int d x x \cdot a=a=f(0) .
\end{aligned}
$$

\section{Def.8: Complex conjugation is given by}

$$
\overline{\left(z+z^{\prime}\right)}=\bar{z}+\bar{z}^{\prime}, \quad \overline{\left(z z^{\prime}\right)}=\bar{z}^{\prime} \bar{z}, \quad \forall z, z^{\prime} \in \Lambda_{\infty} .
$$

Def.9: A supernumber $z$ is called

$$
\text { real : } \Leftrightarrow \bar{z}=z
$$

$$
\text { imaginary : } \Leftrightarrow \bar{z}=-z \text {. }
$$

Note:(i) The generators of $\Lambda_{\infty}$ are choosen as real Grassmannians

$$
\bar{\zeta}^{a}=\zeta^{a}, \quad \forall a .
$$

(ii) The product of two real a-numbers is an imaginary c-number:

$$
\overline{\left(v_{1} v_{2}\right)}=\bar{v}_{2} \bar{v}_{1}=v_{2} v_{1}=-v_{1} v_{2}, \quad \forall v_{1}, v_{2} \in \mathbf{R}_{a} .
$$

Let us introduce the concept of a supervector space.

Def.10: The set $S$ of mappings is called a supervector space if

(i) $\mathrm{S}$ is under the action $+: S \times S \rightarrow S$ an abelian group;

(ii) For all $\alpha \in \Lambda_{\infty}$ and $\vec{x} \in S$ there exist two mappings multiplication from the left $\alpha_{L}: S \rightarrow S, \vec{x} \rightarrow \alpha_{L} \vec{x}$, multiplication from the right $\alpha_{R}: \quad S \rightarrow S, \vec{x} \rightarrow \vec{x} \alpha_{R}$. Furthermore one has for all $\alpha_{L}, \beta_{L} \in \Lambda_{\infty}, \quad \vec{x}, \vec{y} \in S$ :

$$
\begin{aligned}
\left(\alpha_{L}+\beta_{L}\right) \vec{x} & =\alpha_{L} \ddot{x}+\beta_{L} \vec{x} \\
\alpha_{L}(\vec{x}+\vec{y}) & =\alpha_{L} \ddot{x}+\alpha_{L} \vec{y} \\
\left(\alpha_{L} \beta_{L}\right) \vec{x} & =\alpha_{L}\left(\beta_{L} \vec{x}\right) \equiv \alpha_{L} \beta_{L} \vec{x} \\
1 \cdot \vec{x} & =\ddot{x}
\end{aligned}
$$

and analogously for the multiplication from the right

In the following (if not explicetly noted otherwise) multiplication from the left is implicetely assumed and $I$ omit furtheron the index $L$.

(iii)

$$
\begin{array}{cl}
(\alpha \vec{x}) \beta=\alpha(\vec{x} \beta)=\alpha \vec{x} \beta, & \forall a, \beta \in \Lambda_{\infty}, \vec{x} \in S \\
\alpha \vec{x}=\vec{x} \alpha, & \forall a \in \mathbf{C}_{c} \vec{x} \in S \\
a \vec{u}=\vec{u} \alpha, a \vec{x}=-\vec{r} a, & \forall a-\mathbf{C} \cdot \vec{x}=\vec{u}+\vec{r}=S,
\end{array}
$$

where $\vec{u}$ and $\vec{v}$ are the even and odd components of $\vec{x}$, respectively. In anology to the supernumbers one defines pure supervectors of type $c$ and type a, respectively.

For pure supernumbers and supervectors Eq.(18) can be rewritten as

$$
\alpha \vec{x}=(-1)^{\alpha \vec{x}} \vec{x} \alpha .
$$

The expressions in the exponent of Eq.(19) take on the values 0(1), depending on whether the corresponding expression is of type $\mathrm{c}(\mathrm{a})$.

(iv) One has complex conjugation with

$$
\begin{aligned}
\overline{\overline{\vec{x}}} & =\vec{x} \\
\overline{(\vec{x}+\vec{y})} & =\overline{\bar{x}}+\overline{\vec{y}} \\
\overline{\alpha \vec{x}} & =\overline{\vec{x}} \vec{\alpha}
\end{aligned}
$$

for all $\vec{x}, \vec{y} \in S$ and all $\alpha \in \Lambda_{\infty}$.

A supervector $\vec{x} \in S$ can be represented with respect to a basis $\{\vec{i} \vec{\epsilon}\}$ :

$$
\vec{x}=x^{i}{ }_{i} \vec{\epsilon} \quad x^{i} \in \Lambda_{\infty},
$$

where the contraction between two indices can be done according to the rule " $y$ " or "У", respectively. A supervector space has total dimension $\mathbf{d}$, iff it has a basis containing $d$ supervectors. If $d$ is finite, one can always find a basis which contains $m$ real c-type supervectors and $n$ real a-type supervectors. Such a basis is called a pure real basis and the ordered pair $(m, n)$ is called the dimension of the superspace. Both, $d$ and $(m, n)$ are independent from the choice of a basis.

Let $\left\{{ }_{i} \vec{e}\right\}$ and $\left\{{ }_{j} \vec{\epsilon}\right\}$ two real bases. The basis-changing matrix has the block structure

$$
\left({ }_{i} K^{j}\right)=\left(\begin{array}{ll}
A & C \\
D & B
\end{array}\right)
$$

with $A \in \operatorname{Mat}\left(m \times m ; \mathbf{C}_{c}\right), \quad B \in \operatorname{Mat}\left(n \times n: \mathbf{C}_{\varepsilon}\right), \quad \mathbf{C} \in \operatorname{Mat}\left(m \times n ; \mathbf{C}_{a}\right)$ and $D \in \operatorname{Mat}\left(n \times m ; \mathbf{C}_{a}\right)$. Then for $\vec{x} \in S$ :

$$
x^{\prime j}=x^{i}{ }_{i} K^{-j}
$$

A matrix $\mathrm{K}$ with the block structure (22) will be called a standard matrix. Def.11: The shifting of super vector indices is described by

$$
{ }^{i} x:=(-1)^{\bar{x} i} x^{i} .
$$

Def.12: Let $\mathrm{K}$ be a standard matrix. Then

$$
{ }^{j} K_{i}^{*}:=(-1)^{(i+j)}{ }_{i} K^{-j}
$$

is called the supertranspose of $\mathbf{K}$.

With these definitions one has the properties $(\vec{x} \in S)$

$$
\begin{aligned}
x^{i}{ }_{i} K^{-j} & ={ }^{j} K_{i}^{-i} x \\
(K L)^{-} & =L^{-\cdots} K^{-} .
\end{aligned}
$$


Explicitly

$$
\left(\begin{array}{ll}
A & C \\
B & B
\end{array}\right)^{\sim}=\left(\begin{array}{cc}
A^{T} & -D^{T} \\
C^{T} & B^{T}
\end{array}\right)
$$

where $T$ denotes the ordinary transposition of matrices.

Def.13: The shifting of matrix indices is described hy

$$
K_{i}^{j}=(-1)^{i}{ }_{i} K^{j}
$$

Def.14: The supertrace of a standard matrix is defined by

$$
\operatorname{str} K^{-}:=K_{i}{ }^{i}
$$

explicitly:

$$
\operatorname{str} K=\operatorname{tr} A-\operatorname{tr} B .
$$

One has the properties

$$
\begin{aligned}
\operatorname{str}_{(m, n)} & =m-n \\
\operatorname{str} K^{\sim} & =\operatorname{str} K \\
\operatorname{str}(M N) & =\operatorname{str}(N M) .
\end{aligned}
$$

Def.15: The superdeterminant of a standard matrix $M$ is defined by integration of the relation

$$
\delta(\ln \operatorname{sdet} M)=\operatorname{str}\left(M^{-1} \delta M\right)
$$

with the boundary condition

$$
\operatorname{sdet}_{(m, n)} \equiv 1
$$

Explicitly:

$$
\text { sdet }\left(\begin{array}{cc}
A & C \\
D & B
\end{array}\right)=\frac{\operatorname{det}\left(A-C B^{-1} D\right)}{\operatorname{det} B}=\frac{\operatorname{det} A}{\operatorname{det}\left(B-D A^{-1} C\right)}
$$

The superdeterminant of a matrix exists only iff the matrices $A$ and $B$ have non vanishing body.

Without proof I state the inverse of a standard matrix

$$
\begin{aligned}
& \left(\begin{array}{ll}
A & C \\
D & B
\end{array}\right)^{-1} \\
& =\left(\begin{array}{cc}
\left(\mathbf{1}_{m}-A^{-1} C B^{-1} D\right)^{-1} A^{-1} & -\left(\mathbf{1}_{m}-A^{-1} C B^{-1} D\right)^{-1} A^{-1} C B^{-1} \\
-\left(\mathbf{1}_{n}-B^{-1} D A^{-1} C\right)^{-1} B^{-1} D A^{-1} & \left(\mathbf{1}_{n}-B^{-1} D A^{-1} C\right)^{-1} B^{-1}
\end{array}\right)
\end{aligned}
$$

In the next section I discuss supermanifolds and superconformal automorphisms.

\section{Supermanifolds}

To discuss global properties of superspaces I need the notion of supermanifolds. Ordinary manifolds are locally homöomorph to $\mathbf{R}^{n}$, whereas supermanifolds have locally the structure of $\mathbf{R}_{c}^{m} \times \mathbf{R}_{\mathrm{a}}^{n}$.

Def.16: The mapping $\pi: \mathbf{R}_{c}^{m} \times \mathbf{R}_{a}^{n} \rightarrow \mathbf{R}^{m}$, which maps every coordinate $p \in \mathbf{R}_{c}^{m} \times \mathbf{R}_{a}^{n}$ onto its body is called a canonical projection. The set $\pi^{-1}(\pi(x))$ with $x \in \mathbf{R}_{c}^{m} \times \mathbf{R}_{a}^{n}$ is called the soulfiber of $x$.

Def.17: Let $l^{*} \subset \mathbf{R}^{m}$ an open set. $V \subset \mathbf{R}_{c}^{m} \times \mathbf{R}_{a}^{n}$ is an open set only iff it can be represented by $\pi^{-1}\left(C^{*}\right)$.

$\mathbf{R}_{c}^{m} \times \mathbf{R}_{a}^{n}$ with this topology is called a projective Hausdorff space. Two points $x, y \in \mathbf{R}_{c}^{m} \times \mathbf{R}_{a}^{n}$ have only disjunct neighbourhoods iff $x_{B} \neq y_{B}$.

Def.18: Let $M$ be a set, $U_{A} \subset M$ an open subset and $A$ an index set. Let $\Phi_{A}$ be a bijective mapping from $U_{A}$ to an open subset of $\mathbf{R}_{c}^{m} \times \mathbf{R}_{a}^{n} . \quad M$ is called a supermanifold of dimension $(\mathbf{m}, \mathbf{n})$, together with a collection of ordered pairs $\left(U_{A}, \Phi_{A}\right)$, if each $U_{A}$ is a subset of $M$ and its associated $\Phi_{A}$ is one-to-one mapping of $U_{A}$ onto an open subset in $\mathbf{R}_{r}^{m} \times \mathbf{R}_{a}^{n}$. The collection of ordered pairs is required to have the following properties:

(i) $\bigcup_{A} C_{A}=M$

(ii) $\Phi_{A} \circ \Phi_{B}^{-1}$ is differentiable over all nonempty intersections $\left.U_{A}\right\urcorner V_{B}$

The ordered pair $\left(U_{A}, \Phi_{a}\right)$ is called a chart, or a local coordinate patch or simply a coordinate system.

Let $p \in M$. The set $\left.\Phi^{-\mathrm{J}}\left\{\pi^{-1} ; \pi \circ \Phi(p)\right\}\right\}$ is called the soulfiber of $\mathbf{p}$. The soulfibers of all $p \in M$ together with the mapping $\pi \circ \Phi$ form an ordinary manifold $M_{B}$ with dimension $m$, the body of $M$.

Supermanifolds of this kind are called DeWitt-supermanifolds. They have a trivial topology in the direction of the soul coordinates, they are fiber bundles over their body $M_{B}$. The reason for this property is the fact that an open set in $M$ is always the cartesian product of an open set of $\mathbf{R}^{m}$ with the entire space of the soul coordinates. If this restriction is omitted one gets Rogers-supermanifolds which allow a more complicated structure in the soul coordinates [76]. From the point of view of plysics only the DeWitt-supermanifolds are of interest, see Rabin and Crane 71 :

Def.19: Let $\mathcal{F}(M)$ the set of all superanalytic functions $f: M \rightarrow A_{\infty}$. The mapping

$$
\vec{X}: \mathcal{F}(M) \rightarrow \mathcal{F}(M), \quad f \mapsto \vec{X} f
$$

is called a contravariant vectorfeld on $M$ if the following chain rule is valid:

$$
(\vec{X} f)(p)=\left(\vec{X} \Phi^{i}\right)(p)\left[\frac{\partial}{\partial y^{i}} f\left(\Phi^{-1}\left(y_{1}, \ldots, y_{m+n}\right)\right)\right]_{y=\Phi(p)}
$$

$\forall p \in M, f \in \mathcal{F}(M), y \equiv\left(y_{1}, \ldots, y_{m+n}\right) \in \mathbf{R}_{c}^{m} \times \mathbf{R}_{\mathbf{a}}^{n},(U, \Phi)$ chart around $p$.

The set of all contravariant vectorfields is denoted by $\mathcal{X}(M)$.

Since the chain rule is valid one can represent every $\vec{X} \in \mathcal{X}(M)$ by partial differentiation

$$
\vec{X} f=X^{i} \frac{\partial}{\partial y^{i}} \hat{f}, \quad \hat{f} \equiv f \circ \Phi^{-1} .
$$


$X^{i} \equiv \vec{X} y^{i}$ are called the components of $\vec{X}$ in the coordinate system, which is defined by $y^{i} \equiv \Phi^{i}$.

Def.20: Let $\vec{X} \in \mathcal{X}(M)$ and $p \in M$. Then

$$
\vec{X}_{p}: \mathcal{F}(M) \rightarrow \Lambda_{\infty}, \quad f \mapsto\left(\vec{X}^{\prime} f\right)(p)
$$

is called the contravariant vector at $p$. The set of all contravariant vectors is called the tangent space $T_{p} M$.

The space $T_{p} M$ is a supervector space of the same dimension $(m, n)$ as the supermanifold $M$. Let $y_{i}$ be the coordinate functions of the chart at $p$, then $i \vec{e}=\left.\left(\partial / \partial y^{i}\right)\right|_{p}$ is a coordinate basis of $T_{p} M$.

Every complete set of linearly independent elements $a \vec{e} \in T_{p} M$ can be used as a basis of the tangent space. It can be expressed as

$$
{ }_{a} \vec{e}={ }_{a} \vec{e}^{i}\left(\frac{\partial}{\partial y^{i}}\right)_{p}
$$

Such a basis is denoted as a Vielbein.

Let us turn to the study of special supermanifolds of real dimension $(2,2)$ or of complex dimension $(1,1)$. Let the coordinates be $z \in \mathbf{C}_{c}, \theta \in \mathbf{C}_{a}$. In the fermionic string theory one is interested in superconformal symmetry. The notion of superspaces and supermanifolds enables one to represent these symmetry transformations as pure "geometrical" transformations in the coordinates $(z, \theta) \in \mathbf{C}_{c} \times \mathbf{C}_{a}$. I consider the transformation $\left(\epsilon \in \mathbf{C}_{a}\right)$ :

$$
\begin{aligned}
& \tilde{z}=z+\theta \epsilon \\
& \tilde{\theta}=\theta+\epsilon .
\end{aligned}
$$

Lagrangians are constructed from fields and their derivatives. Therefore one is lead to use supersymmetric differential opertators. This is nothing but to choose a Vielbein of complex dimension $(1,1)$, which is invariant under the transformation (40). One rewrites $\mathrm{Eq}_{\mathrm{q}}(40)$ in homogeneous coordinates

$$
\left(\begin{array}{c}
\tilde{z} \\
\dot{\theta} \\
1
\end{array}\right)=\underbrace{\left(\begin{array}{ccc}
1 & -\epsilon & 0 \\
0 & 1 & \epsilon \\
0 & 0 & 1
\end{array}\right)}_{=1_{s}+\epsilon X=\exp (\epsilon X)}\left(\begin{array}{c}
\tilde{z} \\
\theta \\
1
\end{array}\right)
$$

and realises the infinitesimal generator

$$
X=\left(\begin{array}{ccc}
0 & -1 & 0 \\
0 & 0 & 1 \\
0 & 0 & 0
\end{array}\right)
$$

as a differential operator

$$
\begin{gathered}
\mathcal{L}_{X}: \mathcal{F}\left(\mathbf{C}_{c} \times \mathbf{C}_{a}\right) \rightarrow \mathcal{F}\left(\mathbf{C}_{\mathrm{c}} \times \mathbf{C}_{a}\right) . \quad f(z, \theta) \mapsto\left[\frac{d}{d f} f(\exp (\epsilon X)(; \cdot \theta)]_{t=0}\right. \\
\cdots \mathcal{L}_{X}=-\theta \partial_{z}+\partial_{\theta} .
\end{gathered}
$$

This operator obviously has odd parity. Invariance of an operator $D \in T_{p} M$ is now equivalent to the restriction

$$
\left[D, \mathcal{L}_{X}\right]=0
$$

where

$$
[A, B]=A B-(-1)^{A B} B A
$$

is the supercommutator of two operators $A$ and $B$. For the operator $D$ one makes the Ansatz

$$
D=a(z, \theta) \partial_{z}+b(z, \theta) \partial_{\theta}, \quad a, b \in \mathcal{F}\left(\mathbf{C}_{c} \times \mathbf{C}_{a}\right) .
$$

In addition one has the constraints $D^{2} \neq 0$ and $D^{2} \in T_{p} M$. For the supercommutato of $D$ and $\mathcal{L}_{X}$ one gets:

$$
\left[D, \mathcal{L}_{X}\right]=\left(-b-\theta\left(\partial_{z} a\right)+\partial_{\theta} a\right) \partial_{\varepsilon}+\left(-\theta\left(\partial_{z} b\right)+\partial_{\theta} b\right) \partial_{\theta},
$$

which yields for $D$

$$
D=b\left[(a+\theta) \partial_{z}+\partial_{\theta}\right], \quad a \in \mathbf{C}_{a}, b \in \mathbf{C}_{c} .
$$

We choose the normalization $a=0$ and $b=1$, i.e.

$$
D=\theta \partial_{z}+\partial_{\theta} .
$$

Therefore the operator $D$ is something like the square root of $\partial_{z}$ since $D^{2}=\partial_{z}$. Let us consider a general superanalytic coordinate transformation

$$
\begin{aligned}
& \tilde{z}=\tilde{z}(z, \theta) \\
& \tilde{\theta}=\tilde{\theta}(z, \theta) .
\end{aligned}
$$

Then $D$ transforms as follows

$$
\begin{aligned}
& D=(\partial \tilde{z}) \tilde{\partial}_{z}+\left(\partial_{\theta} \tilde{\theta}\right) \tilde{\partial}_{\theta}+\theta\left(\partial_{z} \tilde{z}\right) \bar{\partial}_{z}+\theta\left(\partial_{\tilde{z}} \tilde{\theta}\right) \tilde{\partial}_{\theta} \\
& =\left\{\left(\partial_{\theta} \tilde{\theta}\right)-\theta\left(\partial_{z} \tilde{\theta}\right)\right] \tilde{\partial}_{\theta}+\left[\left(\partial_{\theta} \bar{z}\right) \div \theta\left(\partial_{z} z\right): \tilde{\partial}_{z}\right. \\
& =(D \bar{\theta})\left(\bar{D}-\bar{\theta} \dot{D}^{2}\right)+(D \bar{z}) \bar{D}^{2} \\
& =(D \tilde{\theta}) \tilde{D}+(D \bar{z}-\bar{\theta} D \bar{\theta}) \tilde{D}^{2} \text {. }
\end{aligned}
$$

Def.21: A superanalytic coordinate transformation is called superconformal, iff the (0.1)-dimensional subspace of the tangential space generated by the action of $D$ is invariant under the coordinate transformation, i.e.

$$
D=(D \tilde{\theta}) \tilde{D} .
$$

Def.22: A Super Riemann Surface is a complex $(1,1)$-dimensional supermanifold, whose coordinate transformations are superconformal mappings.

Example: The Super Riemann sphere $S \dot{C}$ is generated by two coordinate systems $Z=(z, \theta)$ and $\bar{Z}=(\Sigma, \bar{\theta})$ which are connected by the superconformal transformation

$$
(\Sigma, \bar{\theta})=-\left(\begin{array}{cc}
1 & \theta \\
\hdashline & \frac{A}{Z}
\end{array}\right)
$$


One introduces homogeneous coordinates and can represent $S \hat{\mathrm{C}}$ as a $(1,1)$-dimensional projective space $\left(\xi, z_{1}, z_{2}\right) \in \mathbf{C}_{a} \times \mathbf{C}_{c}^{2} \backslash\{0\} \equiv \mathbf{P}_{(1,1)}\left(\Lambda_{\infty}\right)$. The local coordinate systems read

$$
(z, \theta)=\left(\frac{z_{1}}{z_{2}}, \frac{\xi}{z_{2}}\right), \quad(\tilde{z}, \tilde{\theta})=\left(\frac{z_{2}}{z_{1}}, \frac{\xi}{z_{1}}\right) .
$$

The group $S P L(2, \mathbf{C})$ of superconformal automorphisms of $S \hat{\mathbf{C}}$ is the natural super-generalisation of the Möbius transformations. ${ }^{1}$ On $P_{(1,1)}\left(\Lambda_{\infty}\right)$ these transformations can be realised as linear transformations, which are superconformal in the local coordinates of $S \hat{\mathbf{C}}$. Also one has the constraint that $S P L(2, \mathbf{C})_{B o d y}=S L(2, \mathbf{C})$ A reasonable Ansatz reads:

$$
\begin{aligned}
S P L\left(1,2 ; \mathbf{C}_{a} \times \mathbf{C}_{c}^{2}\right):=\left\{\gamma=\left(\begin{array}{ccc}
e & \alpha & \beta \\
\delta & a & b \\
\gamma & c & d
\end{array}\right): a, b, c, d, \epsilon \in \mathbf{C}_{c} ;\right. \\
\left.\alpha, \beta, \gamma, \delta \in \mathbf{C}_{a} ; a d-b c=1 ; \text { sdet } \gamma=1\right\} .
\end{aligned}
$$

Locally the transformation

$$
x^{\prime}=\gamma x, \quad x, x^{\prime} \in \mathbf{P}_{(1,1)}\left(\Lambda_{\infty}\right), \quad \gamma \in S P L\left(1,2 ; \mathbf{C}_{a} \times \mathbf{C}_{c}^{2}\right)
$$

reads as

$$
\begin{aligned}
& z^{\prime}=\frac{\delta \theta+a z+b}{\gamma \theta+c z+d} \equiv \frac{A}{B} \\
& \theta^{\prime}=\frac{e \theta+\alpha z+\beta}{\gamma \theta+c z+d} \equiv \frac{\Gamma}{B} .
\end{aligned}
$$

Superconformal invariance gives the constraint:

$$
\begin{gathered}
D z^{\prime}=\theta^{\prime} D \theta^{\prime} \\
\Rightarrow \frac{(D A) B-A(D B)}{B^{2}}=\frac{\Gamma}{B} \frac{(D \Gamma) B+\Gamma(D B)}{B^{2}} \\
\Rightarrow(D A) B-A(D B)=\Gamma(D \Gamma)
\end{gathered}
$$

Comparison of the coefficients yields

$$
\begin{aligned}
& \epsilon^{2}=1+\beta \alpha+2 \gamma \delta \\
& \epsilon=1+(3 / 2) \beta \alpha \\
& e \alpha+c \delta-a \gamma=0, \\
& \Rightarrow \quad \gamma=d \alpha-c \beta, \\
& \epsilon \beta+d \delta-b \gamma=0 \\
& \delta=b \alpha-a \beta
\end{aligned}
$$

Inserting (59) into (57) gives finally

$$
\begin{aligned}
& z^{\prime}=\frac{a z+b}{c z+d}+\theta \frac{\alpha z+\beta}{(c z+d)^{2}}, \\
& \theta^{\prime}=\frac{\alpha z \beta}{c z+d}+\frac{\theta}{c z+d}\left(1+\frac{\beta \alpha}{2}\right) .
\end{aligned}
$$

'Its generators are the operators $L_{0}, L_{\frac{1}{2}}, L_{-\frac{1}{2}}, G_{\frac{1}{2}}$ and $G_{-\frac{1}{2}}$ of the Neveu-Schwarz section of the Virasoro super aigebra of the fermionic string. In Ref. $[3 ; S P L(2, \mathbf{C})$ is denoted as $O S p(2 \mid 1, \mathbf{C})$
I further define the quantities $N_{\gamma}$ and $\chi_{\gamma}$ by

$$
x_{\gamma}\left[N_{\gamma}{ }^{\frac{1}{2}}+N_{\gamma}{ }^{-\frac{1}{2}}\right]=(a+d)\left(1-\frac{\alpha \beta}{2}\right) \cdots \alpha \beta
$$

$N_{\gamma}$ is called the norm of an hyperbolic $\gamma \in \Gamma$ and $\lambda_{\gamma}$ describes the corresponding spin structure. $x_{\gamma}$ can take on the values \pm 1 and has to be chosen as $\lambda_{\gamma}=\operatorname{sign}(a+\dot{d})$. $N_{\gamma_{0}}$ will denote the norm of a primitive $\gamma_{0} \in \Gamma$, where elements $\gamma \in \Gamma$ which are not powers (greater or equal to 2 ) of any element in $\Gamma$ are called prinitive elements of $\Gamma$ in analogy to the usual bosonic case. $\Gamma$ is called a super Fuchsian group, the subgroup $S P L(2, \mathbf{R})$ of $S P L(2, \mathbf{C})$, thus the group of superconformal automorphisms of $S \mathcal{H}$. Its body is the corresponding norm of an element $\gamma_{B} \in P S L(2, \mathbf{R})$, the group of hyperboljc transformations on the Poincaré upper half-plane. In analogy to the classical bosonic case I denote by $l_{\gamma}=\ln N_{\gamma}$ the length of a closed geodesic corresponding to a hyperbolic $\gamma \in \Gamma$. Of course, $l_{\gamma_{0}}$ is the length corresponding to a primitive $\gamma_{0}$. Furthermore, a hyperbolic transformation is always conjugate to the transformation

$$
i^{\prime}=N_{\gamma} z \quad \theta^{\prime}=\lambda_{\gamma} \sqrt{N_{\gamma}} \theta
$$

or in matrix representation:

$$
\text { hyperbolic } \gamma \in \Gamma \text { conjugate to } \quad\left(\begin{array}{ccc}
\chi_{\gamma} & 0 & 0 \\
0 & N_{\gamma}^{\frac{1}{2}} & 0 \\
0 & 0 & N_{\gamma}{ }^{-\frac{1}{2}}
\end{array}\right) \text {. }
$$

To normalize $\gamma$ correctly by sdet $\gamma=1$ one has to multiply all matrix-entries of Eq.(55) by $K=1-\frac{1}{2} \beta \alpha=1+\frac{1}{2} \alpha \beta$. Therefore:

$$
\gamma=K\left(\begin{array}{cccc}
1+\frac{3}{2} \beta \alpha & \alpha & \beta \\
b \alpha-\cdots \beta & a & b \\
d \alpha-c \beta & c & d
\end{array}\right)=\left(\begin{array}{ccc}
1+\beta \alpha & \alpha & \beta \\
b \alpha-a \beta & a\left(1-\frac{1}{2} \beta \alpha\right) & b\left(1-\frac{1}{2} \beta \alpha\right) \\
d \alpha-c \beta & c\left(1-\frac{1}{2} \beta \alpha\right) & d\left(1-\frac{1}{2} \beta a\right)
\end{array}\right)
$$

and with Eq.(34)

$$
\gamma^{-1}=\left(\begin{array}{ccc}
1+\beta \alpha & c \beta-d \alpha & b \alpha-a \beta \\
-\beta & d\left(1-\frac{1}{2} \beta \alpha\right) & -b\left(1-\frac{1}{2} \beta \alpha\right) \\
-\alpha & -c\left(1-\frac{1}{2} \beta \alpha\right) & a\left(1-\frac{1}{2} \beta \alpha\right)
\end{array}\right)
$$

To formulate super uniformisation let us first remember the uniformisation theorem for Riemann surfaces (e.g. $[15 ;)$

\section{Theorem:}

Every compact Riemann surface is conformaly equivalent to $M / \Gamma$, where $M=\mathbf{C}$ (Riemann sphere), $M=\mathbf{C}$ (for the torus) or $M=\mathcal{H}$ (upper half-plane) where $\Gamma$ is a discrete, fix-point free subgroup of the conformal autonorplisms of $M$.

Since $\mathbf{C}, \mathbf{C}$ and $\mathcal{H}$ are simply connected, and super Riemann surfaces are fiber bundles over their body, there exist generalisations $S \dot{\mathbf{C}}, S \mathbf{C}$ and $S \mathcal{H}$. The conformal automorphisms of $\mathbf{C}$ and $\mathcal{H}$ are subgroups of $S L(2, \mathbf{C})$. This is not true in general for the superconformal automorphisms of $S \hat{\mathbf{C}}$ and $S H$. But for application in physies we 
need in general a metric and we can restrict ourselves to "metrizable" super Riemann surfaces. Superconformal automorphisms of a "metrizable" super Riemann surface, which leave the metric invariant, are always subgroups of $S P L(2, \mathrm{C})$.

With the DeWitt definition of open sets, a subgroup $\Gamma \in S P L(2, \mathbf{R})$ acts discrete and without fix-points, iff $\Gamma_{\text {Red }} \equiv \Gamma_{\text {Body }} \subset S L(2, \mathbf{R})$.

Theorem [71]:

Every "metrizable" super Riemann surface $\Sigma$ is superconformally equivalent to $M / \Gamma$ with $M=S \hat{\mathbf{C}}, S \mathbf{C}$ or $S \mathcal{H}$ and $\Gamma$ is a discrete fix-point free subgroup of the superconformal automorphisms on $M$.

The coefficients in Eq.(55) are specified by $a, b, c, d \in \mathbf{R}_{c}$ and $\alpha, \beta \in \mathbf{C}_{a}, \bar{\alpha}=i \alpha$, $\bar{\beta}=i \beta$.

As is well known, the fundamental group of a compact Riemann surface of genus $g$ can be defined by $2 g$ generators satisfying the relation (I.17). In the super case one has analogousily:

$$
\left(\gamma_{0} \gamma_{1}^{-3} \ldots \gamma_{2 g-2} \gamma_{2 g-1}^{-1}\right)\left(\gamma_{0}^{-1} \gamma_{1} \ldots \gamma_{2 g-2}^{-1} \gamma_{2 g-1}\right)=\left(\begin{array}{ccc}
1 & 0 & 0 \\
0 & 1 & 0 \\
0 & 0 & 1
\end{array}\right)
$$

The real Teichmüller space $T$ of a compact Riemann surface with genus $g$ has dimension $d_{\mathcal{T}}=6 g-6$, whereas super Teichmüller space $S \mathcal{T}$ has dimension $d_{S T}=$ $(6 g-6,4 g-4)$.

The simplest generalisation of the Poincaré metric on $\mathcal{H}$ which is $S P L(2, \mathbf{R})$ invariant reads on $S \mathcal{H}$

$$
d s^{2}=\frac{|d z+\theta d \theta|^{2}}{\left(\operatorname{In} z+\frac{1}{2} \theta \tilde{\theta}\right)^{2}},
$$

but this metric turns out not to be invertible!
III. Physics on the Poincaré Super Upper Half-Plane

\section{Classical Motion}

To construct the metric on $S \mathcal{H}$ let us consider the Vierbein $E^{A}$. The general method for constructing the Vierbein in a curved $2+2$-dimensional super space was given by Howe [51]. Because a $2+2$-dimensional super space is conformally flat, if there exists a coordinate system in which the metric is proportional to the flat metric, one starts with the Vierbein $\hat{E}_{M}{ }^{A}$ in flat superspace

$$
\hat{E}_{M}{ }^{A}=\left(\begin{array}{cccc}
1 & 0 & 0 & 0 \\
0 & 1 & 0 & 0 \\
-\theta & 0 & 1 & 0 \\
0 & \bar{\theta} & 0 & 1
\end{array}\right), \quad \hat{E}_{A}{ }^{M}=\left(\begin{array}{cccc}
1 & 0 & 0 & 0 \\
0 & 1 & 0 & 0 \\
\theta & 0 & 1 & 0 \\
0 & -\bar{\theta} & 0 & 1
\end{array}\right),
$$

where $\hat{E}_{A}{ }^{M}=\left(\hat{E}_{M}{ }^{A}\right)^{-1}$ is the inverse Vierbein. This gives for the quantities $\hat{E}^{A}=$ $d z^{M} \hat{E}_{M}{ }^{A}$ :

$$
\begin{array}{ll}
\hat{E}^{z}=d z+\theta d \theta, & \hat{E}^{\theta}=d \theta, \\
\hat{E}^{\bar{z}}=d \bar{z}-\bar{\theta} d \bar{\theta}, & \hat{E}^{\bar{\theta}}=d \bar{\theta} .
\end{array}
$$

Under a super Weyl transformation the Vierbein $\hat{E}_{M}{ }^{A}$ changes as

$$
\hat{E}_{M}{ }^{A} \rightarrow E_{M}{ }^{A}= \begin{cases}E_{M}{ }^{a}=\Lambda(Z) \hat{E}_{M}{ }^{a}, & (a=z, \bar{z}), \\ E_{M}{ }^{a}=\Lambda^{\frac{1}{2}}(Z) \hat{E}_{M}{ }^{a}-i \hat{E}_{M}^{a}\left(\gamma_{a}\right)^{\alpha \beta} D_{\beta} \Lambda^{\frac{1}{2}}(Z), & (\alpha=\theta, \bar{\theta}),\end{cases}
$$

where $D_{\alpha}=E_{\alpha}{ }^{M} \partial_{M}, \Lambda(Z)$ the scaling function and $\left(\gamma_{a}\right)$ the $\gamma$-matrices which in my notation read ${ }^{3}$

$$
\left(\gamma_{\Sigma}\right)^{\alpha \beta}=\left(\begin{array}{cc}
0 & 2 \\
0 & 0
\end{array}\right), \quad\left(\gamma_{\Sigma}\right)^{\alpha \beta}=\left(\begin{array}{ll}
0 & 0 \\
2 & 0
\end{array}\right) .
$$

Since the Vierbein $E^{A}$ should be (up to phase factors) invariant under the action of $S P L(2, \mathbf{R})$ the appropriate scaling function reads $\Lambda(Z)=Y^{-1}$, where $Y$ is given by $\mathrm{Y}:=\operatorname{Im} z+\frac{\theta \tilde{\theta}}{2}=y+i \theta_{1} \theta_{2}$, if $\mathrm{I}$ further set $\theta=\theta_{1}+i \theta_{2}$ and $\bar{\theta}=\theta_{1}-i \theta_{2}$, where $\theta_{1}$ and $\theta_{2}$ are real Grassmannians. Note: $\bar{Y}=Y$. The $E^{A}$ are now given as (see $[\pi 1,86]$ )

$$
\begin{aligned}
& E^{z}=\frac{d z+\theta d \theta}{Y}, \quad E^{\bar{z}}=\frac{d \bar{z}+\bar{\theta} d \bar{\theta}}{Y}, \\
& E^{\theta}=\frac{d \theta}{2 Y^{\frac{3}{2}}}+\frac{i \theta-\bar{\theta}}{Y^{\frac{3}{2}}}(d z+\theta d \theta), \\
& E^{\bar{\theta}}=\frac{d \bar{\theta}}{2 Y^{-\frac{3}{2}}}-\frac{i \theta+\bar{\theta}}{2 Y^{\frac{s}{2}}}(d \bar{\nu}-\bar{\theta} d \bar{\theta}) .
\end{aligned}
$$

In Appendix A the $S P L(2, \mathbf{R})$ invariance of the $E^{A}$ is shown. The $S P L(2, \mathbf{R})$ invariant line element can now be constructed by $[86]:^{2}$

$$
\begin{aligned}
d s^{2} & =E^{z} E^{\bar{z}}-2 E^{\theta} E^{\hat{\theta}} \\
& =\frac{1}{Y^{2}}\left(d z-i \bar{\theta} d \theta^{\prime 2}-2 Y\left(d \theta_{i}^{\prime 2}\right)\right. \\
& =\frac{1}{Y^{-2}}\left(d z d z-i \bar{\theta} d \bar{z} d \theta-i \theta d \bar{\theta} d z-(2 Y+\theta \dot{\theta}) d \theta d \bar{\theta} \bar{j}_{j} .\right.
\end{aligned}
$$

'Raising and lowering of spin-indices are performed by the totally amisymmetric $\epsilon_{a 3^{3}}$-lensor. See the remark following Eq.(44) below. 
Rewriting $d s^{2}=d q^{a}{ }_{a} g_{b} d q^{b}$ one gets the metric tensor on $S \mathcal{H}$

$$
\left(_{a} g_{b}\right)=\frac{1}{2 Y^{2}}\left(\begin{array}{cccc}
0 & 1 & 0 & -i \theta \\
1 & 0 & -i \bar{\theta} & 0 \\
0 & i \bar{\theta} & 0 & -(2 Y+\theta \bar{\theta}) \\
i \theta & 0 & 2 Y+\theta \bar{\theta} & 0
\end{array}\right)
$$

The metric tensor is obviously of the form

$$
\left({ }_{a} g_{b}\right)=\left(\begin{array}{cc}
A & \Gamma \\
-\Gamma^{T} & B
\end{array}\right), \quad A=A^{T}, B=-B^{T} .
$$

Equation (II.33) gives the superdeterminant of $\left({ }_{a} g_{b}\right)$

$$
\operatorname{siet}\left({ }_{a} g_{b}\right)=\frac{\operatorname{det} A}{\operatorname{det}\left(B+\Gamma^{T} A^{-1} \bar{\Gamma}\right)}=-\frac{1}{4 Y^{2}} .
$$

Note that $\left({ }_{a} g_{b}\right)$ is a "super" Kähler metric

$$
\begin{aligned}
{ }_{2} g_{\bar{z}} & =\frac{1}{2 Y^{2}}=\partial_{z} \partial_{\bar{z}} \ln \frac{1}{Y^{2}} \\
{ }_{\bar{\theta}} g_{z} & =\frac{-i \theta}{2 Y^{2}}=\partial_{z} \partial_{\bar{\theta}} \ln \frac{1}{Y^{2}} \\
{ }_{\theta} g_{\bar{\Sigma}} & =\frac{i \bar{\theta}}{2 Y^{2}}=\partial_{\bar{z}} \partial_{\theta} \ln \frac{1}{Y^{2}} \\
{ }_{\theta} g_{\bar{\theta}} & =-\frac{2 Y+\theta \bar{\theta}}{2 Y^{2}}=\partial_{\bar{\theta}} \partial_{\theta} \ln \frac{1}{Y^{2}} .
\end{aligned}
$$

One constructs the $S P L(2, \mathbf{R})$ invariant volume element on $S \mathcal{H}$ as

$$
\begin{aligned}
d V(Z) & =\sqrt{\mid \overline{\operatorname{siet}\left({ }_{a} g_{b}\right) \mid}} d z d \bar{z} d \theta d \bar{\theta} \\
& =\frac{d z d \bar{z} d \theta d \bar{\theta}}{2 Y} .
\end{aligned}
$$

Note the difference in the power of $Y$ to the $P S L(2, \mathbf{R})$ invariant volume element on $\mathcal{H}: d V(z)=d x d y / y^{2}$. In Appendix $\mathrm{A}$ it is shown that $d V(Z)$ is indeed invariant under the action of $S P L(2, \mathbf{R})$. The $S P L(2, \mathbf{R})$ invariant Lagrangian is constructed as (following Refs. $[86,87]$ ):

$$
L=\frac{m}{2}\left(\frac{d s}{d t}\right)^{2}=\frac{m}{2 Y^{2}}[\dot{\bar{z}} \dot{z}-i \bar{\theta} \dot{\bar{z}} \dot{\theta}-i \theta \dot{\theta} \dot{\theta}-(2 Y+\theta \ddot{\theta}) \dot{\theta} \bar{\theta}]
$$

The Euler-Lagrange equations derived from this Lagrangian read

$$
\begin{array}{ll}
\ddot{z}+i \frac{\dot{\tilde{z}}^{2}}{Y}+\frac{\theta \dot{\dot{z}} \dot{\theta}}{Y}=0, & \ddot{\theta}+i \frac{\dot{z} \dot{\theta}}{Y}=0, \\
\ddot{\bar{z}}+i \frac{\dot{\bar{z}}^{2}}{\mathrm{Y}}+\frac{\theta \dot{\bar{z} \dot{\theta}}}{Y}=0, & \ddot{\bar{\theta}}+i \frac{\dot{\bar{\theta}} \overline{\dot{\theta}}}{Y}=0 .
\end{array}
$$

These are geodesic equations

$$
{ }^{a} \ddot{q}+{ }^{a} \Gamma_{b c}{ }^{c} \dot{q}^{b} \dot{q}=0
$$

where

$$
{ }^{a} \Gamma_{b c}=\frac{1}{2} g^{d}\left[(-1)^{(d+1) b)} \frac{\partial_{d} g_{c}}{\partial q^{b}}+(-1)^{(d+b+1) c} \frac{\partial_{d} g_{b}}{\partial q^{c}}-(-1)^{b} \frac{\partial_{b} g_{c}}{\partial q^{d}}\right]
$$

Here $\left({ }^{a} g^{b}\right)=\left({ }_{a} g_{b}\right)^{-1}$ denotes the inverse of the metric tensor and with the help of Eq.(II.33) it is given by

$$
\left({ }^{a} g^{b}\right)=Y\left(\begin{array}{cccc}
0 & 2 Y-\theta \bar{\theta} & 0 & i \bar{\theta} \\
2 Y-\theta \bar{\theta} & 0 & -i \theta & 0 \\
0 & -i \theta & 0 & 1 \\
i \bar{\theta} & 0 & -1 & 0
\end{array}\right) .
$$

Let us assume that the classical solutions of the "pure bosonic" equations

$$
\ddot{z}+i \frac{\dot{\tilde{z}}^{2}}{y}=\ddot{\Xi}-i \frac{\dot{\bar{z}}}{y}=0
$$

have been found, $[z(t), \bar{z}(t)]=\left\{z_{0}(t), \bar{z}_{0}(t)\right]$, then

$$
[z(t), \bar{z}(t), \theta(t), \bar{\theta}(t)]=\left[z_{0}(t), \bar{z}_{0}(t), \epsilon_{1} z_{0}(t)+\epsilon_{2}, \omega\left(\epsilon_{1} \bar{z}_{0}(t)+\epsilon_{2}\right)\right]
$$

are classical solutions of the Euler-Lagrange equations, where $\epsilon_{1}$ and $\epsilon_{2}$ are Grassmann constants and $\omega$ is a constant phase factor with $|\omega|=1$. Explicitly

$$
\begin{aligned}
& z(t)=c_{1} \tanh \omega\left(t-t_{\theta}\right)+c_{2}+i c_{1} \frac{1}{\cosh \omega\left(t+t_{0}\right)}, \\
& \theta(t)=\epsilon_{1} z(t)+\epsilon_{2},
\end{aligned}
$$

where $c_{1}, c_{2}$ and $t_{0}$ are real even numbers and $\epsilon_{1}$ and $\epsilon_{2}$ are odd ones (for more details see $[8 \pi]$ ). It turns out that there exist periodic orbits on bounded domains on $S \mathcal{H}$ and that this motion is chaotic [60]. The corresponding geodesics also obey Huber's law. The super hyperbolic distance between two points $q^{(1)}$ and $q^{(2)}$ on $S \mathcal{H}$ is now defined as

$$
d\left(q^{(1)}: q^{(2)}\right)=\int_{q^{11}}^{q^{(2)}} d s=\int_{t_{1}}^{t_{2}} \sqrt{\left(\frac{d s}{d t}\right)^{2} d t=\omega\left(t_{2}-t_{1}\right) .}
$$

This can be rewritten as

$$
\cosh d\left(q^{(1)}, q^{(2)}\right)=1+\frac{1}{2} R\left(q^{(1)}, q^{(2)}\right)-2 r\left(q^{(1)}, q^{(2)}\right),
$$

where $\left[\right.$ set $\left.q^{(1)} \equiv Z, q^{(2)} \equiv W=\left(u+i v, \nu_{1}+\nu_{2}\right), V=v+\nu \bar{j} / 2\right)$ :

$$
R(Z, W)=\frac{|z-w-\theta \nu|^{2}}{Y \mathfrak{T}}=\frac{\mid z-u-\theta \nu^{\prime 2}}{(y+\theta \bar{\theta} / 2)(r+\nu \ddot{\nu} / 2)}
$$




$$
\begin{array}{r}
r(Z, W)=\frac{2 \theta \bar{\theta}+i(\nu-i \bar{\nu})(\theta+i \bar{\theta})}{4 Y}+\frac{2 \nu \bar{\nu}+i(\theta-i \bar{\theta})(\nu+i \bar{\nu})}{4 \bar{V}} \\
+\frac{(\nu+i \bar{\nu})(\theta+i \bar{\theta}) \operatorname{Re}(z-w-\theta \nu)}{4 Y V} .
\end{array}
$$

All these two-point quantities enjoy the following property

$$
\bullet(\gamma Z, \gamma W)=\bullet(Z, W)=\bullet(W, Z) .
$$

In Appendix A the $S P L(2, \mathbf{R})$ invariance of these quantities is discussed.

Let us note the parity of the matrixelements and the rules for index shifting for the metric tensor and its inverse

$$
\begin{array}{ll}
\mathcal{P}\left({ }_{a} g_{b}\right)=(-1)^{a+b}, & { }_{b} g_{a}=(-1)^{a+b+a b}{ }_{a} g_{b} \\
\mathcal{P}\left({ }^{a} g^{b}\right)=(-1)^{a+b}, & { }^{b} g^{a}=(-1)^{a b a} g^{b} .
\end{array}
$$

Let us turn to the Hamiltonian formulation. We have for the conjugate momenta

$$
{ }_{a} p=\frac{\partial L}{\partial \dot{q}^{a}}=\frac{m}{2}\left[{ }_{a} g_{b}{ }^{b} \dot{q}+(-1)^{a+(b+a) b}{ }_{b} g_{a}{ }^{b} \dot{q}\right]=m_{a} g_{b}{ }^{b} \dot{q} .
$$

Explicitly

$$
\begin{aligned}
& p_{z}=\frac{m}{2 Y^{2}}(\dot{\bar{z}}-i \theta \dot{\bar{\theta}}) \\
& p_{\bar{z}}=\frac{m}{2 Y^{2}}(\dot{z}+i \dot{\theta} \bar{\theta}) \\
& p_{\theta}=\frac{i m}{2 Y^{2}} \bar{\theta}(\dot{\bar{z}}-i \theta \dot{\theta})-m \frac{\dot{\bar{\theta}}}{Y} \\
& p_{\bar{\theta}}=-\frac{i m}{2 Y^{2}} \theta(\dot{z}+i \theta \dot{\bar{\theta}})+m \frac{\dot{\bar{\theta}}}{Y} .
\end{aligned}
$$

Note the important relations $\overline{p_{\bar{z}}}=p_{\overline{\bar{z}}}$ and $\overline{p_{\theta}}=-p_{\bar{\theta}}$. This yields for the velocities

$$
\dot{q}^{a}=\frac{1}{m}{ }^{a}{ }^{b} b p
$$

and therefore for the Hamiltonian:

$$
\begin{aligned}
\mathcal{H}(p, q) & \left.=\dot{q}^{a}{ }_{a} p-\mathcal{L}(q, \dot{q})\right\}_{\dot{q}=\dot{q}(q, p)} \\
& =\frac{1}{2 m}{ }^{a} g^{b}{ }_{b} p_{a} p=\frac{(-1)^{a}}{2 m} p_{a}{ }^{a} g^{b}{ }_{b} p=\mathcal{L}(q, \dot{q}) .
\end{aligned}
$$

\section{Quantization}

Let us consider the quantum mechanics on $S \mathcal{H}$. Since our Lagrangian is a nonlinear one, i.e. the ${ }_{a} g_{b}$ in Eq.(III.7) are functions of the coordinates, the ordering of operators cannot be ignored. Let us start with general considerations. To define position and momentum operators canonical super commutation relations are assumed:

$$
\left[\underline{\mathrm{q}}^{a}, \underline{\mathrm{q}}^{b}\right]=0=\left[\underline{\mathrm{p}}_{a}, \underline{\mathrm{p}}_{b}\right], \quad\left[\underline{\mathrm{p}}_{a}, \underline{\mathrm{q}}^{b}\right\}=-i \hbar_{a} \delta^{b} .
$$

In the $q$-representation $\underline{q}^{a}$ and $\mathrm{p}_{a}$ are given by

$$
\underline{\mathrm{q}}_{a}=q_{a}, \quad \underline{\mathrm{p}}_{a}=-i \hbar g^{-\frac{1}{4}} \partial_{a} g^{-\frac{1}{4}}=-i \hbar\left(\partial_{a}+\frac{1}{2} \Gamma_{a}\right),
$$

where $g=\left|\operatorname{sdet}\left({ }_{a} g_{b}\right)\right|$ and $\Gamma_{a}=\frac{1}{2} \partial_{a} \ln g$ (In the following I omit the bar on the operators and set $\hbar=1$.). Following Ref. $\{86\}$ the super Laplace-Beltrani operator can be constructed as

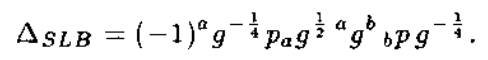

This super Laplacian is a straightforward generalisation of the classical bosonic one and is in general the simplest one which is invariant under general point canonical transformations (see $[42,67,68,86]$ for a discussion of the classical bosonic case). The quantum Hamilionian on a super Riemann manifold is then given by

$$
H=-\frac{1}{2 m} \Delta_{S L B}
$$

More explicitly (do not worry about the position of the indices)

$$
\begin{aligned}
H & =-\frac{1}{2 m}\left[{ }^{a} g^{b} \partial_{b} \partial_{a}+\frac{(-1)^{a}}{\sqrt{g}}\left(\partial_{a} \sqrt{g}^{a} g^{b}\right) \partial_{b}\right] \\
& =-\frac{1}{2 m}{ }^{a} g^{b} \partial_{b} \partial_{a}+(-1)^{a}\left(\Gamma_{a}{ }^{a} g^{b}+\left(\partial_{a}{ }^{a} g^{b}\right)\right) \partial_{b}
\end{aligned}
$$

The ordering prescription (32) is a special one. Another possibility is super Weyl ordering

$$
\left.H=\frac{1}{8 m}{ }^{a} g^{b} p_{b} p_{a}+2(-1)^{a} p_{a}^{a} g^{b} p_{b}+(-1)^{a+b} p_{b} p_{a}{ }^{a} g^{b}\right\rceil+\Delta Y
$$

where the quant um correction $\Delta V$ (of order $\hbar^{2}$ ) is given by

$$
\Delta V=\frac{1}{8 m}\left[{ }^{a} g^{b} \Gamma_{b} \Gamma_{a}+(-1)^{a}\left(\partial_{a}{ }^{a} g^{b} \Gamma_{b}\right)+(-1)^{a+b}\left(\partial_{b} \partial_{a}{ }^{a} g^{b}\right)\right] .
$$

All these formulas are straightforward generalisations of the classical bosonic case 42 . Let us apply these prescriptions to $S \mathcal{H}$. The momenta are given by

$$
\begin{array}{ll}
p_{z}=\frac{1}{i}\left(\frac{\partial}{\partial z}+\frac{i}{4 Y}\right), & p_{\Sigma}=\frac{1}{i}\left(\frac{\partial}{\partial \bar{z}}-\frac{i}{4 Y}\right), \\
p_{\theta}=\frac{1}{i}\left(\frac{\partial}{\partial \theta}-\frac{\bar{\theta}}{4 Y}\right), & p_{\theta}=\frac{1}{i}\left(\frac{\partial}{\partial \bar{\theta}}-\frac{\theta}{4 Y}\right) .
\end{array}
$$


Plugging the momenta into Eq.(35) gives

$$
\begin{aligned}
H & =-\frac{1}{2 m}{ }^{a} g^{b} \partial_{b} \partial_{\alpha} \\
& =-\frac{Y}{m}\left[(2 Y-\theta \bar{\theta}) \partial_{z} \partial_{\bar{z}}+i \bar{\theta} \partial_{z} \partial_{\bar{\theta}}-i \theta \partial_{z} \partial-\theta \bar{\theta} \partial_{\theta} \partial_{\bar{\theta}}\right] .
\end{aligned}
$$

In the first line in (38) one recognizes the same structure as in the bosonic case. where $H=-\frac{1}{2 m}{ }^{a} g^{b} \partial_{b} \partial_{a}=-\frac{1}{2 m} y^{2}\left(\partial_{x}^{2}+\partial_{y}^{2}\right)$. Since the Hamiltonian represents an observable, it should be, at least in a formal sense, hermitian. Therfore one is lead to the following

Def:: The complex conjugate of the partial derivatives on $\mathbf{C}_{c} \times \mathbf{C}_{\boldsymbol{a}}$ are given by

$$
\left(\bar{\partial}_{\Sigma}\right) \equiv \partial_{\bar{z}}, \quad\left(\bar{\partial}_{\theta}\right) \equiv-\partial_{\bar{\theta}} .
$$

It is quite surprising that the Hamiltonian can be factorized. Let us define

$$
\begin{aligned}
& \square=2 Y D \bar{D}=2 Y\left(\partial_{\hat{\theta}} \partial_{\theta}+\theta \bar{\theta} \partial_{z} \partial_{\tilde{z}}-\theta \partial_{\bar{\theta}} \partial_{z}-\bar{\theta} \partial_{\theta} \partial_{z}\right), \\
& D=\partial_{\theta}+\theta \partial_{\Sigma}, \quad \bar{D}=-\partial_{\bar{\theta}}+\bar{\theta} \partial_{\Sigma}
\end{aligned}
$$

[i.e. $D=D_{\theta}, \bar{D}=D_{\bar{\theta}}$, in the Vierbein notation of Eq.(1)]. Then we have the important relation:

$$
\Delta_{S L B}=\square^{2}
$$

Proof:

$$
\begin{aligned}
\square^{2} & =4 Y D \bar{D} Y D \bar{D} \\
& =4 Y D[(\bar{D} Y) D \vec{D}+Y \bar{D} D \bar{D}] \\
& =4 Y\left[(D \bar{D} Y) D \bar{D}-(\bar{D} Y) D^{2} \bar{D}+(D Y) \bar{D} D \bar{D}+Y D \bar{D} D \dot{D}\right] .
\end{aligned}
$$

One makes use of the relations

$$
\begin{aligned}
D \bar{D} & =-\bar{D} D, \quad D^{2}=\partial_{z}, \quad \bar{D}^{2}=-\partial_{\bar{z}}, \\
D Y & =-\frac{i}{2}(\theta+i \bar{\theta}), \quad \bar{D} Y=\frac{1}{2}(\theta+i \bar{\theta}), \\
D \bar{D} Y & =i D^{2} Y=i \partial_{z} Y=\frac{1}{2}
\end{aligned}
$$

and get

$$
\square^{2}=-\frac{Y}{m}\left[(2 Y-\theta \bar{\theta}) \partial_{z} \partial_{\bar{z}}+i \bar{\theta} \partial_{z} \partial_{\bar{\theta}}-i \theta \partial_{z} \partial-\theta \bar{\theta} \partial_{\theta} \partial_{\bar{\theta}} \bar{j},\right.
$$

which completes the proof

It is quite interesting to note that this possibility of taking the square roote of $\triangle_{S L B}$ crucially depends on the choice of the factor "2" in $d s^{2}$ of Eq.(6). Demanding $\Delta_{S L B}=$ $\square^{2}$ determines this factor unambiguousiy.

$\square$ is a $S P L(2, \mathbf{R})$ invariant operator.

Proof: In Appendix $\mathrm{A}$ it is shown that one has for the $S P L(2, \mathbf{R})$ transformation (II.60) the following transformation properties

$$
\begin{aligned}
& D=\frac{1}{\hat{B}(Z)} D^{\prime} \\
& Y=|\hat{B}(Z)|^{2} Y^{\prime} .
\end{aligned}
$$

Therefore

$$
\square=2 Y D \hat{D}=2 \dot{B}(Z)^{2} \frac{1}{\hat{B}(Z)} D^{\prime} \frac{1}{\hat{\bar{B}}(Z)} \bar{D}^{\prime}=2 Y^{\prime} D^{\prime} \bar{D}^{\prime}=\square^{\prime}
$$

Generally I refer to the operator $\square$ as the Laplace-Dirac operator on $S \mathcal{H}$

With the invariant volume element on $S \mathcal{H}, \Delta_{S L B}$ and $\square$ are hermitean with respect to the scalar product

$$
\left(\Phi_{1}, \Phi_{2}\right)=\int d V(Z) \Phi_{1} \bar{\Phi}_{2}
$$
by ${ }^{1}$

The operator $\square$ is the zero-case of the more general operator $\square_{m}$ which is defined

$$
\square \square_{m}=2 Y D \bar{D}+m(i \theta-\bar{\theta}) \bar{D} .
$$

This is the important opcrator for the fermionic string (see next chapter). In Ref.[10] also the operator $\hat{\square}_{m}$ is introduced which is constructed by a linear isomorphism

$$
\square_{m}=Y^{\frac{m}{2}}\left(\hat{\square}_{m}+\frac{m}{2}\right) Y^{-\frac{m}{2}} .
$$

Hence we have an unitary equivalence of $\square_{m}$ and $\hat{\square}_{m}+\frac{m}{2}$. Explicitly $\hat{\square}_{m}$ reads:

$$
\begin{aligned}
\hat{\square}_{m} & =2 Y D \bar{D}+\frac{m}{2}(i \theta-\bar{\theta})(\bar{D}+i D) \\
& =2 Y D \bar{D}-i \frac{m}{2}\left(\theta_{1}+\theta_{2}\right)\left(\partial_{\theta_{1}}-\partial_{\theta_{2}}\right)-i \frac{m}{2} \theta \bar{\theta} \partial_{x} .
\end{aligned}
$$

I denote this unitary equivalence by $\square_{m} \cong \hat{\varphi}+\frac{m}{2}$. Let us consider an even differentiable superfunction on $S \mathcal{H}$ ( $A, B$ even, $x, \bar{X}$ odd)

$$
\Phi(z, \Sigma, \theta, \bar{\theta})=A(z, \bar{z})+\frac{1}{\sqrt{y}}[\theta \chi(z, \bar{z})+\bar{\theta} \bar{x}(z, \bar{z})]+\frac{1}{y} \theta \bar{\theta} B(z, \bar{z})
$$

With the notation $-\Delta_{m}=-4 y^{2} \partial_{z} \partial_{\Sigma}+i m y \partial_{x}=-y^{2}\left(\partial_{x}^{2}+\partial_{y}^{2}\right)+i m y \partial_{x}$ one gets the following equivalence relation $\left[10^{0}\right.$ :

$$
\hat{\square}_{m} \Phi=s \Phi \Leftrightarrow\left\{\begin{aligned}
-\Delta_{-m} A & =s(1-s) A, \quad B=\frac{s}{2} A \\
-\Delta_{-m-1} \chi & =\left(\frac{1}{4}-s^{2}\right) \chi, \quad-\Delta_{-m+1} \bar{\lambda}=\left(\frac{1}{4}-s^{2}\right) \bar{\chi}, \\
\bar{\chi}\left(s-\frac{m}{2}\right) & =-2 y \partial_{\Sigma} \chi-\frac{i}{2}(m-1) \chi,
\end{aligned}\right.
$$

where $s$ is an even supernumber. Thus, the solution of the Eigenvalue problem is formally the same as the classical bosonic one. However, the periodic boundary conditions [for e.g. $m=0: \psi(\gamma Z)=\psi(z), \gamma \in S P L(2, \mathbf{R})$ ] must be interpreted in the super language. By taking the body in all quantities, one recovers, of course, the old problem. The equivalence relation legitimates to set $s=\frac{1}{2}+i p(p \in \mathbf{R}$, so called "small" Eigenvalues neglected). This reproduces the positivity of the operator $-\Delta_{m}$.

${ }^{1}$ use a slightly different notation as in Baranov et al. $\{10,11\}$ and Aoki [3]. In Refs. $\{3,10]$ a deseription is given, how such opertors can be constructed in a systematic approach. 
An odd superfunction is constructed in taking the quantities $A, B, \chi$ and $\tilde{\chi}$ in Eq.(51) odd and even, respectively.

As is easily checked, $\Phi_{1}=Y^{s}$ and $\Phi_{2}=\left(\theta_{1}+\theta_{2}\right) y^{-s}$ satisfy (52), i.e. $\Phi_{1}$ and $\Phi_{2}$ are an even and an odd solution of the Laplace-Dirac operator $\hat{\emptyset}_{m}$, respectively, with Eigenvalue $s:^{1}$

$$
\widehat{\mathrm{Q}}_{m} \boldsymbol{\Phi}_{i}=s \Phi_{i}, \quad(i=1,2) .
$$

Let us note the squares of the Laplace-Dirac operators $\square_{m}$ and $\hat{Q}_{m}$. They read

$$
\begin{aligned}
\square_{m}^{2}= & {\left[\square_{0}+m(i \theta-\bar{\theta}) \bar{D}\right]^{2} } \\
= & \square_{0}^{2}+m \square_{0}(i \theta-\bar{\theta}) \bar{D}+m(i \theta-\bar{\theta}) \bar{D} \square_{0}+m^{2}(i \theta-\bar{\theta}) \bar{D}(i \theta-\bar{\theta}) \bar{D} \\
= & \square_{0}^{2}+2 m Y\left(D \bar{D}+i \partial_{\bar{z}}\right)+m^{2}(i \theta-\bar{\theta}) \bar{D} ; \\
\hat{\square}_{m}^{2}= & {\left[\square_{0}+\frac{m}{2}(i \theta-\bar{\theta})(\bar{D}+i D)\right]^{2} } \\
= & \square_{0}^{2}+\frac{m}{2} \square_{0}(i \theta-\bar{\theta})(\bar{D}+i D)+\frac{m}{2}(i \theta-\bar{\theta})(\bar{D}+i D) \square_{0} \\
& \quad+\frac{m^{2}}{4}(i \theta-\bar{\theta})(\bar{D}+i D)(i \theta-\bar{\theta})(\bar{D}+i D) \\
& \quad \square_{0}^{2}+i m Y \partial_{x} .
\end{aligned}
$$

Let us finally discuss quantization in the path integral formalism on a $(m, n)$-dimensional super Riemann manifold. I state just the most important results. A more detailed treatment is not discussed here. Let us consider the Eigenstate $|q\rangle$ of the coordinate operator $q$ with Eigenvalue $q$ and the properties

$$
\left.<q\left|q^{\prime}>=\right| g(q) g\left(q^{\prime}\right)\right]^{-\frac{1}{4}}\left(q^{\prime}\right) \delta\left(q-q^{\prime}\right), \quad \mathbf{1}=\int d q \sqrt{g}|q><q| .
$$

Now define the kernel function $K\left(q^{\prime \prime}, q^{\prime} ; t^{\prime \prime}, t^{\prime}\right)$ describing the time evolution

$$
\Psi\left(q^{\prime \prime} ; t^{\prime \prime}\right)=\int d q \sqrt{g} h^{\prime}\left(q^{\prime \prime}, q^{\prime} ; t^{\prime \prime}, t^{\prime}\right) \Psi\left(q^{\prime} ; t^{\prime}\right)
$$

by the matrix element

$$
K\left(q^{\prime \prime}, q^{\prime} ; t^{\prime \prime}, t^{\prime}\right)=\left\langle q^{\prime \prime}\left|\varepsilon^{-i\left(t^{\prime \prime}-t^{\prime}\right) H_{s w}}\right| q^{\prime}>.\right.
$$

Here the super Weyl ordering prescription in the Hamiltonian (c.f. Eq.(35)) is chosen. I proceed in the usual manner, i.e. subdividing the time interval $T=t^{\prime \prime}-t^{\prime}$ into $N$. subintervals of equal length $\epsilon=T / N$ and let $t^{(k)}=t^{\prime}+\epsilon k, q^{(k)}=q\left(t^{(k)}\right)(k=$ $0,1, \ldots, N)$. First one gets

$$
\begin{aligned}
H^{\prime}\left(q^{\prime \prime}, q,: T\right) & =<q^{\prime \prime}\left|\epsilon^{-i\left(t^{\prime \prime}-t^{\prime}\right) H_{S w}}\right| q^{\prime}> \\
& =\left(\prod_{j=1}^{N^{\prime}-1} \int \sqrt{g^{(j)}} d q^{(j)}\right) \times \prod_{j=1}^{N}<q^{(j)}\left|\exp \left[-i \epsilon H_{S W^{\prime}}\right]\right| q^{(j-1)}>.
\end{aligned}
$$

'Note that an Eigenfunction of the form $\phi=A+\frac{\theta \vec{b}}{u} B$ which is satisfying the relation (52) $\left(\gamma^{\prime}\right.$ is a special case of such an Eigenfunction) has under the assumption $s=\frac{1}{2}+i p(p \in \mathbf{R})$ with respect to the scatar-product (4i) zero-norm. Even worse, in general the scalar-product (4i) does not form a Hilbert space inner product in the sense that it is not positive definite - quite puzzeling in view of

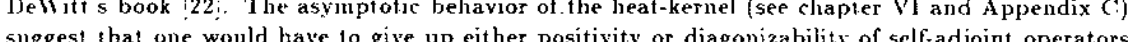
suggest that one would have to give up either positivity or diagonizability of selfadjoint operators
for botly), to have a super reparametrization invariant notion of super Hither1 space " 4 .
The short-time matrix element can be evaluated $\left[q_{M}^{(j)}=\frac{1}{2}\left(q^{(j)}+q^{(j-1)}\right), \Delta q^{(j)}=\right.$ $\left.q^{(j)}-q^{(j-1)}, d p=d p_{x} d p_{y} d p_{\theta} d p_{\bar{\theta}}\right]:$

$$
\begin{aligned}
& <q^{(j)}\left|e^{-i \epsilon H_{S W}}\right| q^{(j-1)}> \\
& \simeq 1-i \epsilon<q^{(j)}\left|H_{S W}\right| q^{(j-1)}>\simeq\left[g\left(q^{\prime}\right) g\left(q^{\prime \prime}\right)\right]^{-\frac{1}{4}} \int \frac{d p^{(j)}}{(2 \pi)^{m}} \\
& \times \exp \left\{i\left[p^{(j)} \Delta q^{(j)}-\frac{\epsilon}{2 m}{ }^{a} g^{b}\left(q_{M}^{(j)}\right) p_{b}^{(j)} p_{a}^{(j)}-\epsilon V\left(q_{M}^{(j)}\right)-\epsilon \Delta V\left(q_{M}^{(j)}\right)\right]\right\}
\end{aligned}
$$

Here use has been made of the matrix elements

$$
<z\left|p_{z}>=\frac{1}{2 \pi} \int d p_{z} e^{i p_{z} z}, \quad<\theta\right| p_{\theta}>=\frac{1}{i} \int d p_{\theta} \epsilon^{i p_{\theta} \theta},
$$

where $z$ denotes any of the commuting variables and $\theta$ any of the anticommuting variables. Equations (62) imply

$$
\begin{aligned}
& \int d p_{\theta} d p_{\bar{\theta}} e^{i p_{\theta}\left(\theta^{\prime \prime}-\theta^{\prime}\right)} e^{i p_{\bar{\theta}}\left(\bar{\theta}^{\prime \prime} \ldots \bar{\theta}^{\prime}\right)} \\
& =\int d p_{\theta} d p_{\bar{\theta}}\left[1+i p_{\theta}\left(\theta^{\prime \prime}-\theta^{\prime}\right)+i p_{\bar{\theta}}\left(\bar{\theta}^{\prime \prime}-\bar{\theta}^{\prime}\right)+p_{\theta} p_{\bar{\theta}}\left(\bar{\theta}^{\prime \prime}-\bar{\theta}^{\prime}\right)\left(\theta^{\prime \prime}-\theta^{\prime}\right)\right] \\
& +\left(\bar{\theta}^{\prime \prime}-\bar{\theta}^{\prime}\right)\left(\theta^{\prime \prime}-\theta^{\prime}\right)=\delta\left(\bar{\theta}^{\prime \prime}-\bar{\theta}^{\prime}\right) \delta\left(\theta^{\prime \prime}-\theta^{\prime}\right)
\end{aligned}
$$

Now the integration formula for Gaussian integrals in superspace [22, p.46, p.304i is used:

$$
\int d q \exp \left[\frac{i}{2} q_{a}^{a} M_{b} M^{b} q-i p_{a}{ }^{a} q_{?}^{?}=\frac{(2 \pi i)^{\frac{m}{2}}}{|\operatorname{sdet} M|^{\frac{1}{2}}} e^{\frac{i}{2}{ }^{a} M^{b}{ }_{b} p_{a} p} .\right.
$$

Here it is assume that $\left({ }_{a} M_{b}\right)$ is of the block diagonal form

$$
\left({ }_{a} M_{b}\right)=\left(\begin{array}{cc}
A & C \\
-C^{T} & B
\end{array}\right)
$$

where $A$ is a matrix with Eigenvalues whose bodies are strictly positive and $B$ a matrix of the block form

$$
B=\operatorname{diag}\left[\left(\begin{array}{cc}
0 & i \mu_{2} \\
-i \mu_{1} & 0
\end{array}\right) \ldots,\left(\begin{array}{cc}
0 & i \mu_{n} \\
-i \mu_{n} & 0
\end{array}\right)\right]
$$

with $\mu_{i}>0(i=1, \ldots, n)$. Otherwise additional phases occur in (64) - see [22]. Modified for the inverse of $\left(a M_{b}\right) .\left({ }^{a} M^{b}\right)$ the Gaussian integral reads (and note that I handle the variabie $q$ and $p$ as a standard basis):

$$
\int d p \exp \left[\frac{i}{2} p_{a}{ }^{a} M H^{b} p_{b}-i q^{a}{ }_{a} p^{3}\right]=\frac{(2 \pi i)^{\frac{m}{2}}}{|\operatorname{sdet} M|^{\frac{1}{2}}} e^{\frac{i}{2} q^{a}{ }_{a} g_{k}{ }^{b} q} .
$$

iPhases due to negative Eigenvalues in the matrix $A$ are not allowed in ordinary quantum mechanics. the underlying body of the discussed theory. Additional phases due to negative $\mu_{i}$ read as follows For every $\mu_{i} \because 0$ multilpy (64) by the factor $i$. 
Therefore the $p$-integrations can be carried out. Finally one has to take the limit $N \rightarrow \infty$ and the path integral on a $(m, n)$-dimensional super Riemann manifold reads

$$
\begin{aligned}
& \left.\quad K^{\prime \prime}, q^{\prime} ; T\right)=\left[g\left(q^{\prime}\right) g\left(q^{\prime \prime}\right)\right]^{-\frac{1}{4}} \lim _{N^{\prime} \rightarrow \infty}\left(\frac{i^{m-2 n}}{(2 \pi)^{m}}\right)^{\frac{N}{2}} \prod_{j=1}^{N-1} d q^{(j)} \\
& \quad \times \prod_{j=1}^{N} \sqrt{g\left(q_{M}^{(j)}\right)} \exp \left\{i\left[\frac{m}{2 \epsilon} \Delta q^{(j), a}{ }_{a} g_{b}\left(q_{M}^{(j)}\right) \Delta q^{(j)}+\epsilon V\left(q_{M}^{(j)}\right)+\Delta V\left(q_{M}^{(j)}\right)\right]\right\}
\end{aligned}
$$

A detailed proof of this representation will not be given here.

In case of $S \mathcal{H}$ one has $\Delta V=0$ and finds explicitly for the path integral on $S \mathcal{H}$ $\left[Z=(z, \theta)=\left(x+i y, \theta_{1}+i \theta_{2}\right)\right]$

$$
\begin{gathered}
K\left(Z^{\prime \prime}, Z^{\prime} ; T\right)=2 \sqrt{Y^{\prime} Y^{\prime \prime}} \lim _{N \rightarrow \infty}\left(\frac{1}{4 \pi i}\right)^{N} \prod_{j=1}^{N-1} \int d x^{(j)} d y^{(j)} d \theta_{1}^{(j)} d \theta_{2}^{(j)} \prod_{j=1}^{N} \frac{1}{Y_{M}^{(j)}} \\
\times \exp \left\{\frac { i m } { 2 \epsilon Y _ { M } ^ { ( j ) ^ { 2 } } } \left[\Delta z^{(j)} \Delta \bar{z}^{(j)}-i \bar{\theta}_{M}^{(j)} \Delta \bar{z}^{(j)} \Delta \theta^{(j)}-i \theta_{M}^{(j)} \Delta z^{(j)} \Delta \tilde{\theta}^{(j)}\right.\right. \\
\left.\left.-\left(2 Y_{M}^{(j)}+\theta_{M}^{(j)} \bar{\theta}_{M}^{(j)}\right) \Delta \theta^{(j)} \Delta \bar{\theta}^{(j)}\right]\right\} .
\end{gathered}
$$

In a rather tedious calculation it can be directly shown that this is the correct path integral on $S \mathcal{H}$. See Appendix B for some details.

The path integral (68) has not been calculated explicitly up to now. However, there exists a semiclassical solution by Uehara and Yasui [87] and a calculation of the heat kernel $\tilde{K}(T)$ by Aoki $[3]$ by solving directly the equation

$$
\left(\frac{\partial}{\partial t^{\prime \prime}}-\frac{1}{2 m} \square_{m}^{\prime 2}\right) \tilde{K^{\prime}}(T)=\delta\left(q^{\prime \prime}-q^{\prime}\right), \quad\left(T=t^{\prime \prime}-t^{\prime}>0\right)
$$

with the boundary condition

$$
\vec{K}(T) \rightarrow \frac{1}{2 Y^{\prime}} \delta\left(x^{\prime \prime}-x^{\prime}\right) \delta\left(y^{\prime \prime}-y^{\prime}\right) \delta\left(\theta^{\prime \prime}-\theta^{\prime}\right) \delta\left(\bar{\theta}^{\prime \prime}-\bar{\theta}^{\prime}\right), \quad\left(T \rightarrow 0^{+}\right) .
$$

A short summary of Aoki's results is given in Appendix C.
IV. The Selbarg Supertrace Formula for Super Riemann Surfaces

Let us consider the $S P L(2, \mathbf{R})$ transformation as given in Chapter II

$$
\begin{aligned}
& z^{\prime}=\frac{\delta \theta+a z+b}{\gamma \theta+c z+d} \equiv \frac{A}{B}, \\
& \theta^{\prime}=\frac{e \theta+\alpha z+\beta}{\gamma \theta+c z+d} \equiv \frac{\Gamma}{B},
\end{aligned}
$$

where

$$
\begin{gathered}
A(Z)=a z+b-\theta \delta, B(Z)=c z+d-\theta \gamma, \Gamma(Z)=\alpha z+\beta+\epsilon \theta ; \\
\epsilon=1+\frac{3}{2} \beta \alpha, \gamma=d \alpha-c \beta, \delta=b \alpha-a \beta .
\end{gathered}
$$

The numbers $a, b, c, d$ satisfy the relation $a d-b c=1$ and are real even supernumbers. The numbers $\alpha$ and $\beta$ are odd supernumbers with the property $\bar{\alpha}=i a, \bar{\beta}=i \beta$. I also use the notation $Z^{\prime}=\left(z^{\prime}, \theta^{\prime}\right)=\gamma(z, \theta)=\gamma Z$. $A, B$ and $\Gamma$ must be multliplied by $K^{-}=1-\frac{1}{2} a \beta$ to give the correct normalization sdet $T=1$ (see Eq.(II.64)). I denote these quantities by $\hat{A}, \dot{B}$ and $\hat{\Gamma}$, respectively. Let us introduce some important notions:

Def. 1: Let $\Gamma \subset S P L(2, \mathbf{R})$ be a discrete subgroup and $U \subset S \mathcal{H}$ a fundamental domain of $\Gamma$ which tesselates $S \mathcal{H}$.

Def 2: Let,$\in \Gamma$. A function $f(Z)(Z \in S \mathcal{H})$ is called a super automorphic function of weight $m$ iff it is satisfying the relation $f(\gamma Z)=j_{\gamma}^{m}(Z) f(Z)$, where $j_{\gamma}^{m}$ is given by

$$
\begin{aligned}
& j_{\gamma}^{m}=F_{\gamma}^{m}\left|F_{\gamma}\right|^{-m}=\left(\frac{F_{\gamma}}{\vec{F}_{\gamma}}\right)^{\frac{m}{2}}, \\
& F_{\gamma}=D \theta^{\prime}=\frac{1}{\hat{B}(Z)}, \quad\left(z^{\prime}, \theta^{\prime}\right)=\gamma(z, \theta) .
\end{aligned}
$$

The task is to construct the relevant operator for the super trace formula which maps super automorphic functions into super automorphic functions. Let us consider the integral operator $L$

$$
L \phi(Z)=\int_{S \mathcal{H}} d V^{\prime}(W) k_{m}(Z, W) \phi(W)
$$

$L$ is called the Selberg super integral operator on $S \mathcal{H}$, where $k_{m}(Z, W)$ is the integral-kernel of an operator valued function of the operator $\square_{m}$.

Now introduce the functions $\Phi(x)$ and $\Psi(x)$ sufficiently decreasing at $\propto$. Since the Laplace-Dirac operator $\square$ is a $S P L(2, \mathbf{R})$ invariant operator its integral kernel (and the integral kernel of functions of $\square)$ must depend on $S P L(2, \mathbf{R})$ invariant quantities. Therefore one makes the Ansatz

$$
k(Z, W)=k_{0}(Z, W)=\Phi[R(Z, W)]-r(Z, W) \Psi[R(Z, W)] .
$$

The invariants $r(Z, W)$ and $R(Z, W)$ have been defined in Eqs.(III.22). For the heat kernel of the operator $\square^{2}$ the integral kernel $k_{\text {heat }}$ can be explicitly calculated $\{3\rceil$ and has the form of Eq.(6). Let be $m \in \mathbf{N}_{0}$. Introduce the quantity $J^{m}$ :

$$
J^{m}(Z, W)=\left(\frac{z-\ddot{u}+i \theta \bar{\nu}}{\bar{z}-u+i \bar{\theta}_{\nu}}\right)^{\frac{m}{2}}, \quad(Z, W \in S \mathcal{H})
$$


and study the transformation properties of $J^{m}$ for $J^{m}(Z, W) \rightarrow J^{m}(\gamma Z, \gamma W)$. Using Eq.(A.8)

$$
\gamma(z-\bar{w}+i \theta \bar{\nu})=(z-\bar{w}+i \theta \bar{\nu}) \frac{1-\alpha \beta}{B(Z) \bar{B}(W)}=\frac{z-\bar{w}+i \theta \bar{\nu}}{\hat{B}(Z) \overline{\hat{B}}(W)},
$$

one gets the transformation rule for $J^{m}$ :

$$
\begin{aligned}
J^{m}(\gamma Z, \gamma W) & =\left(\frac{z^{\prime}-\bar{w}^{\prime}+i \theta^{\prime} \bar{\nu}^{\prime}}{\overline{z^{\prime}}-w^{\prime}+i \bar{\theta}^{\prime} \nu^{\prime}}\right)^{\frac{m}{2}} \\
& =\left[\frac{\bar{B}(Z)}{\bar{B}(Z)} \cdot \frac{z-\bar{w}+i \theta \bar{\nu}}{\bar{z}-\bar{w}+i \bar{\theta} \nu} \cdot \frac{B(W)}{\bar{B}(W)}\right]^{\frac{m}{2}}=j_{\gamma}^{m}(Z) J^{m}(Z, W) j_{\gamma}^{-m}(W) .
\end{aligned}
$$

Defining now

$$
k_{m}(Z, W)=J^{m}(Z, W) k(Z, W)
$$

and $k_{m}$ has the required properties for the integral kernel of the operator $L$. Thus one formulates the following

Theorem 1: Let $f$ be a super automorphic function with $f \in L^{2}(S \mathcal{H})$ and scalar product $\left[f_{1}, f_{2} \in L^{2}(S \mathcal{H})\right]$

$$
\left(f_{1}, f_{2}\right)=\int d V(Z) f_{1}(Z) \bar{f}_{2}(Z)
$$

The Selberg super integal operator is then given by

$$
L f(z)=\int_{S \mathcal{H}} d V(Z) J^{m}(Z, W)\left\{\Phi[R(Z, W)]-r(Z, W) \Psi\left[R\left(Z, W^{\prime}\right)\right]\right\} f(W)
$$

and maps super automorphic functions into super automorphic functions. Together with the substitution $W^{\prime}=\gamma W^{\prime}$ one easily shows

$$
\begin{aligned}
g(\gamma Z) & =\int_{S \mathcal{H}} d V^{\prime}\left(W^{\prime}\right) k_{m}(\gamma Z, W) f\left(W^{\prime}\right)=\int_{S \mathcal{H}} d V\left(W^{\prime \prime}\right) k_{m}\left(\gamma Z, \gamma W^{\prime}\right) f\left(\gamma W^{\prime \prime}\right) \\
& =\int_{S \mathcal{H}} d V\left(W^{\prime \prime}\right) j_{\gamma}^{m}(Z) k_{m}\left(Z, W^{\prime}\right) j_{\gamma}^{-m}\left(W^{\prime}\right) j_{\gamma}^{m}\left(W^{\prime \prime}\right) f\left(W^{\prime}\right) \\
& =j_{\gamma}^{m}(Z) \int_{S \mathcal{H}} d V^{\prime}\left(W^{\prime}\right) k_{m}\left(Z, W^{\prime}\right) f(W)=j_{\gamma}^{m}(Z) g(Z) .
\end{aligned}
$$

Let $f$ be a super automorphic function and $g=L f$. Then:

$$
\begin{aligned}
& g(Z)=\int_{S \mathcal{H}} d V(W) k_{m}(Z, W) f(W)=\sum_{\{\}_{p}} \int_{\gamma U} d V\left(W^{\prime}\right) k_{m}(Z, W) f(W) \\
& =\sum_{\{,\}_{r}} \int_{r:} d V\left(W^{r}\right) k_{m}(Z, \gamma W) f(\gamma W)=\sum_{\{\neg\}_{r}} \int_{l} d V^{r}\left(W^{r}\right) k_{m}(Z, \gamma W) j_{\gamma}^{m}\left(W^{*}\right) f(W) \\
& =\int_{r} d r(W) h(Z \cdot W) f(W)
\end{aligned}
$$

where

$$
K(Z, W) \equiv \sum_{\{\gamma\}_{p}} k_{m}(Z, \gamma W) j_{\gamma}^{m}(W)
$$

is the super automorphic kernel.

Let us consider the supertrace of $L . L$ represents an integral operator of an operator valued function $h$ of the Dirac operator $\square_{m}$, i.e. $L \cong h\left(\square_{m}\right)$. Let us as sume that the Hilbert space $L^{2}(S \mathcal{H})$ can be mapped onto $l_{S}^{2}:=\{$ the space of all sequences $\left\{a_{n}\right\} \subset \Lambda_{\infty}$ with the property that $\lim _{N \rightarrow \infty} \sum_{n=0}^{N}\left|a_{n}\right|^{2}$ exists $\}$. This assumption is nothing but a generalization to the super case of the well-known theorem that every Hilbert space is isomorph to $l^{2}$. In $l_{S}^{2} \square_{m}$ can be repesented as an infinite dimensional matrix. Let us denote $\left(\square_{m}\right)$ as this matrix representation. Let us furtheron consider the Eigenvalue problem

$$
\left(\square_{m}\right) \Psi=\lambda \Psi
$$

where $\Psi \in l_{S}^{2}$ with appropriate boundary conditions. $\left(\square_{m}\right)$ diagonolized yields $\left(\square_{m}\right) \rightarrow \Lambda=\operatorname{diag}\left(\lambda_{0}^{B}, \lambda_{1}^{B}, \ldots, \lambda_{0}^{F}, \lambda_{1}^{F}, \ldots\right)$, where $\lambda_{n}^{B(F)}$ are the $\mathrm{B}($ ose $)$ - and $\mathrm{F}$ (ermi) Eigenvalues of $\left(\square_{m}\right)$ for the even and odd Eigenfunctions of $\left(\square_{m}\right)$, respectively. Clearly

$$
\operatorname{str} \square_{m}=\operatorname{str}\left(\square_{m}\right)=\operatorname{str} \Lambda=\sum_{n=0}^{\infty}\left(\lambda_{n}^{B}-\lambda_{n}^{F}\right) ;
$$

this expression must be in general regularized (e.g. by the zeta-function method). Let us consider further powers of $\square_{m}: \square_{m}^{k}(k \in \mathbf{N})$. Then

$$
\operatorname{str} \square_{m}^{k}=\operatorname{str}\left(\square_{m}\right)^{k}=\operatorname{str} \Lambda^{k}=\sum_{n=0}^{\infty}\left[\left(\lambda_{n}^{B}\right)^{k}-\left(\lambda_{n}^{F}\right)^{k}\right] .
$$

Since every well-behaved function $h(z)$ can be approximated by powers of $z$, one finally gets for $\operatorname{str}(L)$ on the one hand,

$$
\operatorname{str}(L)=\operatorname{str}\left[h(\square m):=\sum_{n=0}^{\infty}\left[h\left(\lambda_{n}^{b}\right)-h\left(\lambda_{n}^{F}\right)\right]\right.
$$

on the other hand, one has for the transformation $W=\gamma Z=\left(N_{\gamma} z, \lambda_{\gamma} \sqrt{N}_{\gamma}^{-} \theta\right)$;

$$
j_{\gamma}^{m}=\frac{F_{2}^{m}}{\mid F_{\gamma}{ }^{m}}=\left(\frac{D_{\lambda_{\gamma}} \sqrt{N_{\gamma}} \theta}{\left|D_{\lambda \gamma} \sqrt{N_{\gamma}} \theta\right|}\right)^{m}=\chi_{\gamma}{ }^{m} .
$$

Thus we get for $\operatorname{str}(L)$ :

$$
\operatorname{str}\{L)=\int_{L:} d V(Z) H(Z, Z)=\sum_{\{\gamma\}_{p}} \int_{L:} d V(Z) k_{m}(Z, \gamma Z) j_{\gamma}^{m}(Z) \equiv \sum_{\{\gamma\}_{p}} X_{\gamma}^{m} A(\gamma)
$$

where $A(\gamma)$ is given by

$$
\begin{aligned}
& A(\gamma)=\int_{U} d V(Z) k_{m}(Z ; \gamma Z) \\
& =\int \frac{d x d y d \theta d \bar{\theta}}{2 Y} J^{m}(Z, \gamma Z)\{\Phi(R) \sim r T(R)] \\
& =\frac{1}{2} \int_{1}^{N_{\omega}} d y \int_{-\infty}^{\infty} d x \int \frac{d \theta d \vec{\theta}}{y+\frac{\theta \bar{\theta}}{2}} J^{m}(Z, \gamma Z)[\Phi(R)-r \Psi(R)] .
\end{aligned}
$$


Inmediately the term corresponding to the identity transformation can be stated $\left[J^{m}(Z, Z)=(-1)^{\frac{m}{2}}=i^{m}\right]$

$$
A_{0}^{(m)} \equiv A(I)=\frac{i^{m}}{2} \int_{1}^{N_{\supset 0}} d y \int_{-\infty}^{\infty} d x \int \frac{d \theta d \bar{\theta}}{Y} \Phi(0)=i^{m} \pi(g-1) \Phi(0),
$$

since $\operatorname{vol}\left(R S_{g}\right)=4 \pi(g-1)$, where $R S_{g}$ denotes a Riemann surface of genus $g$

For the explicit evaluation of (22) we need the following

Theorem 2: Let $L$ be the super Selberg operator and $\phi$ any Eigenfunction of $\hat{\square}_{m}$ in $S \mathcal{H}$ with $\hat{\square}_{m} \phi=s \phi$. Then

$$
\int_{S \mathcal{H}} d V(Z) k_{m}(W, Z) \phi(Z)=h(s) \phi(W),
$$

where the superfunction $h$ depends only on $s$ and the kernel $k$. The value of $h(s)$ is thus independent of the function $\phi$.

Proof: In Ref.[10] the proof of this theorem is given for all function $\phi$ (even and odd), all $m \in \mathbf{Z}$ and all $W \in S \mathcal{H}$. Since we need theorem 2 only at a specific value, i.e. $W=Z_{0}=(i, 0)$, I restrict myself to that relatively easy case, which has also the advantage that the case of odd functions drops out. Thus for $W=Z_{0}=(i, 0)$ :

$$
\begin{aligned}
J^{m}(i, Z) & =\left(\frac{i-\bar{z}}{-i-z}\right)^{\frac{m}{2}}=\left(\frac{x-i(y+1)}{x+i(y+1)}\right)^{\frac{m}{2}}, \\
R(i, Z) & =\frac{|i-z|^{2}}{y+\frac{\theta \bar{\theta}}{2}}=\frac{x^{2}+(y-1)^{2}}{y}\left(1-\frac{\theta \bar{\theta}}{2 y}\right) \equiv R_{i}\left(1-\frac{\theta \bar{\theta}}{2 y}\right), \\
r(i, Z) & =\frac{\theta \bar{\theta}}{2 y} \\
Y^{s-1} & =\left(y+\frac{\theta \bar{\theta}}{2}\right)^{s-1}=y^{s-1}+\frac{s-1}{2} y^{s-2} \theta \bar{\theta} .
\end{aligned}
$$

Let $\phi$ an even superfunction as in $\mathrm{Eq}_{\mathrm{q}}$ (III.51) without linear in $\theta, \bar{\theta}$-terms, i.e. $\phi$ is of the form $\phi=A\left(1+\frac{s}{2 y} \theta \bar{\theta}\right)$. Insertion yields:

$$
\begin{aligned}
& L \phi\left(Z_{0}\right)=\int d V(Z) k_{m}\left(Z_{0}, Z\right) \phi(Z) \\
= & \frac{1}{2} \int_{0}^{\infty} d y \int_{-\infty}^{\infty} d x \int \frac{d \theta d \bar{\theta}}{Y} J^{m}(i, Z)\{\Phi[R(i, Z)]-r(i, Z) \Psi\{R(i, Z)]\}\left(1+\frac{s}{2 y} \theta \bar{\theta}\right) A(z, \bar{z}) \\
= & \frac{1}{2} \int_{0}^{\infty} \frac{d y}{y} \int_{-\infty}^{\infty} d x\left(\frac{x-i(y+1)}{x+i(y+1)}\right)^{\frac{m}{2}} A(x, y) \\
& \quad \times \int d \theta \bar{\theta}\left[\Phi\left(R_{i}\right)+\frac{s-1}{2 y}-\theta \bar{\theta} \Phi\left(R_{i}\right)-\frac{\theta \bar{\theta}}{2 y} R_{i} \Phi^{\prime}\left(R_{i}\right)-\frac{\theta \bar{\theta}}{2 y} \Psi\left(R_{i}\right)\right] \\
= & \frac{1}{4} \int_{0}^{\infty} \frac{d y}{y^{2}} \int_{-\infty}^{\infty} d x\left(\frac{x-i(y+1)}{x+i(y+1)}\right)^{\frac{m}{2}} A(x, y) \underbrace{\left[-(s-1) \Phi\left(R_{i}\right)+R_{i} \Phi^{\prime}\left(R_{i}\right)+\Psi\left(R_{i}\right)\right]}_{=\tilde{\Phi}\left(R_{i}\right)} \\
= & \frac{1}{4} \int_{0}^{\infty} \frac{d y}{y^{2}} \int_{-\infty}^{\infty} d x\left(\frac{x-i(y+1)}{x+i(y+1)}\right)^{\frac{m}{2}} A(x, y) \tilde{\Phi}\left(R_{i}\right) .
\end{aligned}
$$

Since $\Phi$ depends only on $R_{0}(z, i)$, where $R_{0}=z \frac{w !^{2}}{y !}$ is an $S L(2, \mathbf{R})$ invariant quantity, the last equation can be interpreted in terms of the Selberg trace formula for automorphic forms of weight $m[49]$ with integral kernel $\tilde{\Phi}$. Now, an operator $\tilde{L}$ on the Poincaré upper half-plane whose kernel depends only on $R_{0}$ is in fact a function of the Laplace operator $\Delta_{m}$. It follows that $\bar{L}$ multiplies $\phi$ by

$$
h(s):=\int_{0}^{\infty} y^{s-\frac{3}{2}} \hat{Q}\left(y+y^{-1}-2\right) d y,
$$

where $\hat{Q}$ is given by

$$
\vec{Q}(y)=\int_{0}^{\infty} \dot{\Phi}\left(x^{2}+y\right) d x
$$

This completes the proof.

Let us turn to the calculation of $A(\gamma)$. The invariants $r$ and $R$ are given with the hyperbolic transformation $W^{*}=\gamma Z=\left(N_{\gamma} z, x, \sqrt{N_{\gamma}} \theta\right)$ as:

$$
\begin{aligned}
& R(Z, \gamma Z)=\frac{\left(N_{\gamma}-1\right)^{2}\left(x^{2}+y^{2}\right)}{N_{\gamma} y^{2}}\left(1-\frac{\theta \bar{\theta}}{2}\right), \\
& r(Z, \gamma Z)=\left(2-\chi_{\gamma} N_{\curlyvee}{ }^{\frac{1}{2}}-\lambda_{\gamma} N_{\gamma}{ }^{-\frac{1}{2}}\right) \frac{\theta \bar{\theta}}{2 y}
\end{aligned}
$$

For the $J^{m}$ term one gets:

$$
\begin{aligned}
J^{m}(Z, \gamma Z)= & \left(\frac{z-N_{\gamma} z+i \chi_{\gamma} \sqrt{N_{\gamma}} \theta \bar{\theta}}{\bar{z}-N_{\gamma} z+i \chi_{\gamma} \sqrt{N_{\gamma}} \theta \bar{\theta}}\right)^{\frac{m_{2}}{2}} \\
= & {\left[\left(\frac{x\left(N_{\gamma}-1\right)-i y\left(N_{\gamma}+1\right)}{x\left(N_{\gamma}-1\right)+i y\left(N_{\gamma}+1\right)}-\frac{i \chi_{\gamma} \sqrt{N_{\gamma}} \theta \bar{\theta}}{x\left(N_{\gamma}-1\right)+i y\left(N_{\gamma}+1\right)}\right)\right.} \\
& \left.\quad \times\left(1-\frac{i_{\lambda_{\gamma}} \sqrt{N_{\gamma}} \theta \bar{\theta}}{x\left(N_{\gamma}-1\right)+i y\left(N_{\gamma}+1\right)}\right)\right]^{\frac{m}{2}} \\
= & \left(\frac{x\left(N_{\gamma}-1\right)-i y\left(N_{\gamma}+1\right)}{x\left(N_{\gamma}-1\right)+i y\left(N_{\gamma}+1\right)}\right)^{\frac{m}{2}}\left(1-i m \frac{x\left(N_{\gamma}-1\right) \lambda_{\gamma} \sqrt{N_{\gamma}} \theta \bar{\theta}}{x^{2}\left(N_{\gamma}-1\right)^{2}+y^{2}\left(N_{\gamma}+1\right)^{2}}\right)
\end{aligned}
$$

(Substitution $x=y \xi$ )

$$
=\left(\frac{\xi\left(N_{\gamma}-1\right)-i\left(N_{\gamma}+1\right)}{\xi\left(N_{\gamma}-1\right)+i\left(N_{\gamma}+1\right)}\right)^{\frac{m}{2}}\left(1-\frac{i m \xi X_{\gamma}}{y} \frac{\sqrt{ } N_{\gamma}\left(N_{\gamma}-1\right) \theta \bar{\theta}}{\xi^{2}\left(N_{\gamma}-1\right)^{2}+\left(N_{\gamma}+1\right)^{2}}\right)
$$

(Substitution $\xi=\sqrt{\frac{N,}{N_{r}-1}} \zeta$ )

$$
=\left(\frac{\zeta-2 i \cosh \frac{\ln N_{\gamma}}{2}}{\zeta+2 i \cosh \frac{\ln N_{\gamma}}{2}}\right)^{\frac{m}{2}}\left(1-\frac{i m \zeta \chi_{\gamma}}{y} \frac{\theta \bar{\theta}}{\zeta^{2}+4 \cosh ^{2} \frac{\ln N_{\gamma}}{2}}\right) .
$$

Setting $\ln N_{\gamma}=u$ and finally

$$
J^{m}(Z, \gamma Z)=\left(\frac{\zeta-2 i \cosh \frac{u}{2}}{\zeta-2 i \cosh \frac{u}{2}}\right)^{\frac{m}{2}}\left(1-\frac{i \theta \bar{\theta}}{y} \frac{m \zeta \chi_{\urcorner}}{\zeta^{2}+4 \cosh ^{2} \frac{u}{2}}\right)
$$


$\left(\zeta=\sqrt{\frac{N_{\gamma}-1}{N_{\gamma}}} \frac{y}{x}, u=\ln N_{\gamma}\right)$

We know that $Y^{s}$ is an Eigenfunction of $\widehat{\square}_{m}$ with Eigenvalue $\lambda=s$. Setting $W=$ $Z_{0}=(i, 0)$, then $Y_{0}^{s}=1$. Theorem 2 gives therefore the multiplication by the function $h(s):$

$$
\begin{gathered}
h(s)=\frac{1}{2} \int_{0}^{\infty} d y \int_{-\infty}^{\infty} d x \int \frac{d \theta d \bar{\theta}}{Y} J^{m}(i, Z)\{\Phi[R(i, Z)]-r(i, Z) \Psi[R(i, Z)]\} Y^{s} \\
=\frac{1}{2} \int_{0}^{\infty} d y \int_{-\infty}^{\infty} d x \int d \theta d \bar{\theta}\left(y^{s-1}+\frac{s-1}{2} y^{s-2} \theta \bar{\theta}\right)\left(\frac{x-i(y+1)}{x+i(y+1)}\right)^{\frac{m}{2}} \\
\times\left\{\Phi\left[\frac{x^{2}+(y-1)^{2}}{y}\left(1-\frac{\theta \theta}{2 y}\right)\right]-\frac{\theta \bar{\theta}}{2 y} \Psi\left[\frac{x^{2}+(y-1)^{2}}{y}\left(1-\frac{\theta \theta}{2 y}\right)\right]\right\} \\
=\frac{1}{4} \int_{0}^{\infty} d y \int_{-\infty}^{\infty} d x\left(\frac{x-i(y+1)}{x+i(y+1)}\right)^{\frac{m}{2}} y^{s-2}\left[-(s-1) \Phi\left(\frac{x^{2}+(y-1)^{2}}{y}\right)\right. \\
\left.+\frac{x^{2}+(y-1)^{2}}{y} \Phi^{\prime}\left(\frac{x^{2}+(y-1)^{2}}{y}\right)+\Psi\left(\frac{x^{2}+(y-1)^{2}}{y}\right)\right], \quad(31)
\end{gathered}
$$

where the $(m / 2)^{n t h}$ power is to be a principle value (see [49], p.454). Performing in the $y$-integral a partial integration for $\operatorname{Re}(s)>1$ :

$$
\begin{aligned}
& -(s-1) \int_{0}^{\infty} d y y^{s-2}\left(\frac{x-i(y+1)}{x+i(y+1)}\right)^{\frac{m}{2}} \Phi\left(\frac{x^{2}+(y-1)^{2}}{y}\right) \\
& =\int_{0}^{\infty} d y y^{s-1}\left(\frac{x-i(y+1)}{x+i(y+1)}\right)^{\frac{m}{2}} \\
& \quad \times\left[\Phi^{\prime}\left(\frac{x^{2}+(y-1)^{2}}{y}\right) \frac{x^{2}+(y-1)^{2}}{y}-\frac{i m x}{x^{2}+(y+1)^{2}} \Phi\left(\frac{x^{2}+(y-1)^{2}}{y}\right)\right] .
\end{aligned}
$$

Therefore for $h(s)$ :

$$
\begin{aligned}
& h(s)=\frac{1}{4} \int_{0}^{\infty} d y \int_{-\infty}^{\infty} d x y^{s-2}\left(\frac{x-i(y+1)}{x+i(y+1)}\right)^{\frac{m}{2}} \\
& x\left[\Psi\left(\frac{x^{2}-(y-1)^{2}}{y}\right)+2(y-1) \Phi^{\prime}\left(\frac{x^{2}+(y-1)^{2}}{y}\right)-\frac{i m x y}{x^{2}+(y+1)^{2}} \Phi\left(\frac{x^{2}+(y-1)^{2}}{y}\right)\right]
\end{aligned}
$$

(Substitutions $x=\sqrt{y} \xi$ and $y=\epsilon^{u}$ )

$$
\begin{aligned}
& =\frac{1}{4} \int_{-\infty}^{\infty} d u \int_{-\infty}^{\infty} \frac{d \xi \frac{\left(\xi-2 i \cosh \frac{u}{2}\right)^{m}}{\left(\xi^{2}+4 \cosh \frac{u}{2}\right)^{\frac{m}{2}}} \epsilon^{u\left(s-\frac{1}{2}\right)}\left[\Psi\left(\xi^{2}+4 \sinh ^{2} \frac{u}{2}\right)\right.}{} \\
& \left.\quad \div 2\left(\epsilon^{u}-1\right) \Phi^{\prime}\left(\xi^{2}+4 \sinh ^{2} \frac{u}{2}\right)-\frac{i m \xi \xi^{\frac{u}{2}}}{\xi^{2}+4 \cosh \frac{u}{2}} \Phi\left(\xi^{2}+4 \sinh ^{2} \frac{u}{2}\right)\right]
\end{aligned}
$$

The $\xi$-integral is splitted into two parts in order to project out the even $\xi$-contributione: further the albbreviation $a^{m}(\xi, u)=\left(\xi-2 i \cosh \frac{\|{ }^{m}}{2 m}\right.$ is introduced ( note that $\alpha^{m}$ is an even function in $u$ ):

$$
\begin{aligned}
& h(s)=\frac{1}{4} \int_{-\infty}^{\infty} d u e^{u\left(s-\frac{1}{2}\right)} \int_{0}^{\infty} d \xi \\
& \times\left\{\frac{\Psi\left(\xi^{2}+4 \sinh ^{2} \frac{u}{2}\right)+2\left(e^{u}-1\right) \Phi^{\prime}\left(\xi^{2}+4 \sinh ^{2} \frac{u}{2}\right)}{\left(\xi^{2}+4 \cosh ^{2} \frac{u}{2}\right)^{\frac{m}{2}}}\left[\alpha^{m}(\xi, u)+\alpha^{m}(-\xi, u)\right]\right. \\
& \left.\quad-\frac{i m \xi e^{\frac{u}{2}}}{\xi^{2}+4 \cosh ^{2} \frac{u}{2}} \frac{\Phi\left(\xi^{2}+4 \sinh ^{2} \frac{u}{2}\right)}{\xi^{2}+4 \sinh ^{2} \frac{u}{2}}\left[\alpha^{m}(\xi, u)-\alpha^{m}(-\xi, u)\right]\right\} \\
& \text { (Substitutions } \left.x=\xi^{2}+4 \sinh ^{2} \frac{u}{2}\right) \\
& =\frac{1}{8} \int_{-\infty}^{\infty} d u e^{u\left(s-\frac{1}{2}\right)} \int_{4 \sinh ^{2} \frac{u}{2}}^{\infty} \frac{d x}{(x+4)^{\frac{m}{2}}} \\
& \quad \times\left[\frac{\Psi(x)+2\left(e^{u}-1\right) \Phi(x)}{\sqrt{x-4 \sinh ^{2} \frac{u}{2}}}\left[\alpha^{m}(\xi(x), u)+\alpha^{m}(-\xi(x), u)\right]\right. \\
& \left.\quad-i m e^{\frac{u}{2}} \Phi(x) \frac{\alpha^{m}(\xi(x), u)-\alpha^{m}(-\xi(x), u)}{x+4}\right] . \quad \text { (33) }
\end{aligned}
$$

Thus for appropriate $h$ the operator $h\left(\widehat{D}_{m}\right)$ equals to an integral operator $L$ of the form (5) whose integral kernel $k_{m}(Z, W)$ is related to $h$ by the equations

$$
\begin{aligned}
h(s) & =\int_{-\infty}^{\infty} d u \epsilon^{u\left(s-\frac{1}{2}\right)} g(u), \quad\left(s=\frac{1}{2}+i p\right), \\
g(u) & =\frac{1}{2 \pi} \int_{-\infty}^{\infty} d p e^{-i u p} h\left(\frac{1}{2}+i p\right), \\
g(u) & =\frac{1}{8} \int_{4 \sinh ^{2} \frac{u}{2}}^{\infty} \frac{d x}{(x+4)^{\frac{m}{2}}} \\
& \times\left[\frac{\Psi(x)+2\left(\epsilon^{u}-1\right) \Phi(x)}{\sqrt{x-4 \sinh ^{2} \frac{u}{2}}}\left(a_{+}^{m}+\alpha_{-}^{m}\right)-i m e^{\frac{u}{2}} \Phi(x) \frac{a_{+}^{m}-\alpha_{-}^{m}}{x+4}\right],
\end{aligned}
$$

where the abbreviation $\left.a_{ \pm}^{m}=\alpha^{m} \mid \pm \xi(x), u\right]$ has been used. Since $\square_{m}$ and $\hat{\square}_{m}+\frac{m}{2}$ are unitary equivalent, one can study traces of $\hat{\square}_{m}$ instead of $\square_{m}$. But some care is needed. If one goes back to $\square_{m}$, which is the relevant operator in the fermionic string, then $Y^{s-\frac{m}{2}}$ is an Eigenfunction of $\square m$ with Eigenvalue $\lambda=s$; therefore

$$
\begin{gathered}
\hat{\square}_{m} Y^{s}=s Y=\left(\hat{\square}_{m}+\frac{m}{2}\right) Y^{s}=\left(s \div \frac{m}{2}\right) Y^{s} \\
=\square_{m} Y^{s-\frac{m}{2}}=\left(\hat{\square}_{m}+\frac{m}{2}\right) Y^{r-\frac{m}{2}}=s Y^{-s}
\end{gathered}
$$

Considering now $h$ as an operator valued function of $\square_{m}$ one has $h\left(\square_{m}\right) \cong h\left(\hat{\square}_{m}+\frac{m}{2}\right)$. Therefure one has to replace in the calculation of $h(s)$ as a multiplier of the kernel of $h(\square m) Y^{* s}$ by $Y^{s-\frac{m}{2}}$. Considering $s$ as an Eigenvalue of $\hat{\square}_{m}$. this yields for the nultiplier of the kernel of $h\left(\square_{m}\right)$

$$
\begin{gathered}
h\left(s+\frac{m}{2}\right)=\int_{-\infty}^{\infty} d u \epsilon^{u\left(s-\frac{1}{2}\right)} g(u) . \\
g(u)=\frac{1}{2 \pi} \int_{-\infty}^{\infty} d p \epsilon^{-i u p} h\left\{i p \ldots \frac{m+1}{2}\right) .
\end{gathered}
$$


where $g(u)$ is explicitly given in terms of $\Phi$ and $\Psi$ as in Eq.(34). Note that the contributions $\frac{m}{2}$ coming from $h(s+m / 2)$ and $Y^{s-\frac{m}{2}}$ cancel. To distinguish between the functions $h$ in Eqs. (34) and (36) I often denote $h$ in Eq.(36) by $h_{m}(s) \equiv h\left(s+\frac{m+1}{2}\right)$. Let us consider several combinations of $g(u)$ and $g(-u)$ for later use

$g(u)+g(-u)$

$=\frac{1}{4} \int_{4 \sinh ^{2} \frac{u}{2}}^{\infty} \frac{d x}{(x+4)^{\frac{m}{2}}}\left[\frac{\Phi(x)+4 \sinh ^{2} \frac{u}{2} \Phi^{\prime}(x)}{\sqrt{x-4 \sinh ^{2} \frac{u}{2}}}\left(\alpha_{+}^{m}+\alpha_{-}^{m}\right)-i m \cosh \frac{u}{2} \Phi(x) \frac{\alpha_{+}^{m}-\alpha_{-}^{m}}{x+4}\right]$

$g(u) e^{-\frac{u}{2}}+g(-u) e^{\frac{u}{2}}$

$$
=\frac{1}{4} \int_{4 \sinh ^{2} \frac{u}{2}}^{\infty} \frac{d x}{(x+4)^{\frac{m}{2}}}\left[\frac{\cosh \frac{u}{2} \Psi(x)}{\sqrt{x-4 \sinh ^{2} \frac{u}{2}}}\left(\alpha_{+}^{m}+\alpha_{-}^{m}\right)-i m \Phi(x)^{\frac{\alpha_{+}^{m}}{+}-\alpha_{-}^{m}} \frac{\alpha_{+}^{m}}{x+4}\right]
$$

$g(u)-g(-u)$

$$
=\frac{1}{4} \sinh \frac{u}{2} \int_{4 \sinh ^{2} \frac{u}{2}}^{\infty} \frac{d x}{(x+4)^{\frac{m}{2}}}\left[\frac{4 \cosh \frac{u}{2} \Phi^{\prime}(x)}{\sqrt{x-4 \sinh ^{2} \frac{u}{2}}}\left(\alpha_{+}^{m}+\alpha_{-}^{m}\right)-i m \Phi(x) \frac{\alpha_{+}^{m}-\alpha_{-}^{m}}{x+4}\right](39)
$$$$
g(u) e^{-\frac{u}{2}}-g(-u) e^{\frac{u}{2}}=\frac{1}{4} \sinh \frac{u}{2} \int_{4 \sinh ^{2} \frac{u}{2}}^{\infty} \frac{d x}{(x+4)^{\frac{m}{2}}} \frac{4 \Phi^{\prime}(x)-\Psi(x)}{\sqrt{x-4 \sinh ^{2} \frac{u}{2}}}\left(\alpha_{+}^{m}+\alpha_{-}^{m}\right)
$$

I have now the relevant terms to calculate $A(\gamma)$ :

$$
\begin{aligned}
& A(\gamma)=\frac{1}{2} \int_{1}^{N_{\gamma 0}} d y \int_{-\infty}^{\infty} d x \int \frac{d \theta d \bar{\theta}}{2 Y} \times J^{m}(Z, \gamma Z)\{\Phi[R(Z, \gamma Z)]-r(Z, \gamma Z) \Psi\{R(Z, \gamma Z)]\} \\
&=\frac{1}{2} \int_{1}^{N_{\gamma 0}} d y \int_{-\infty}^{\infty} d x \int d \theta d \bar{\theta}\left(\frac{1}{y}-\frac{\theta \bar{\theta}}{y^{2}}\right)\left(\frac{x\left(N_{\gamma}-1\right)-i y\left(N_{\gamma}+1\right)}{x\left(N_{\gamma}-1\right)+i y\left(N_{\gamma}+1\right)}\right)^{\frac{m}{2}} \\
& \times\left(1-i m \frac{x\left(N_{\gamma}-1\right) \chi_{\gamma} \sqrt{N_{\gamma}} \theta \bar{\theta}}{x^{2}\left(N_{\gamma}-1\right)^{2}+y^{2}\left(N_{\gamma}+1\right)^{2}}\right) \\
& \times\left[\Phi\left(R_{0}\right)-\frac{\theta \bar{\theta}}{y} R_{0} \Phi^{\prime}\left(R_{0}\right)-2\left(1-\chi_{\gamma} \cosh \frac{u}{2}\right) \frac{\theta \bar{\theta}}{2 y} \Psi\left(R_{0}\right)\right]
\end{aligned}
$$

Performing the $\theta \vec{\theta}$-integration and the substitution $x=y \xi$

$=\frac{1}{2} \int_{1}^{N_{\curlywedge 0}} \frac{d y}{y} \int_{-\infty}^{\infty} d \xi\left(\frac{\xi\left(N_{\gamma}-1\right)-i\left(N_{\gamma}+1\right)}{\xi\left(N_{\gamma}-1\right)+i\left(N_{\gamma}+1\right)}\right)^{\frac{m}{2}}$

$\times\left[R_{0} \Phi^{\prime}\left(R_{0}\right)+\left(1-\chi_{\gamma} \cosh \frac{u}{2}\right) \Psi\left(R_{0}\right)+\frac{1}{2} \Phi\left(R_{0}\right)+\frac{i m \xi\left(N_{\gamma}-1\right) \chi_{\gamma} \sqrt{N_{\gamma}} \theta \dot{\theta}}{\xi^{2}\left(N_{\gamma}-1\right)^{2}+\left(N_{\gamma}+1\right)^{2}} \Phi\left(R_{0}\right)\right]$.

Here another substitution was performed, $\xi=\zeta \sqrt{N_{\gamma}} /\left(N_{\gamma}-1\right)=\zeta /\left(2 \sinh \frac{u}{2}\right)$, where
$N_{\gamma} \equiv \epsilon^{u}$ and $R_{0}=\zeta^{2}+4 \sinh ^{2} \frac{u}{2}$. The $y$-integration yields

$$
\begin{aligned}
& A(\gamma)=\frac{\ln N_{>0}}{4 \sinh ^{2} \frac{u}{2}} \int_{-\infty}^{\infty} d \zeta\left(\frac{\zeta-2 i \cosh \frac{u}{2}}{\zeta+2 i \cosh \frac{u}{2}}\right)^{\frac{m}{2}} \\
& \times\left[\left(\zeta^{2}+4 \sinh ^{2} \frac{u}{2}\right) \Phi^{\prime}\left(\zeta^{2}+4 \sinh ^{2} \frac{u}{2}\right)+\left(1-\chi_{\gamma} \cosh \frac{u}{2}\right) \Psi\left(\zeta^{2}+4 \sinh ^{2} \frac{u}{2}\right)\right. \\
& \left.\quad+\frac{1}{2} \Phi\left(\zeta^{2}+4 \sinh ^{2} \frac{u}{2}\right)+\frac{i m \zeta \chi_{\gamma}}{\zeta^{2}+4 \cosh ^{2} \frac{u}{2}} \Phi\left(\zeta^{2}+4 \sinh ^{2} \frac{u}{2}\right)\right] .
\end{aligned}
$$

Let us consider the $\Phi$ and $\Phi^{\prime}$-terms in (41) and perform a partial integration in $\zeta$

$$
\begin{gathered}
\int_{-\infty}^{\infty} d \zeta\left(\frac{\zeta-2 i \cosh \frac{u}{2}}{\zeta+2 i \cosh \frac{u}{2}}\right)^{\frac{m}{2}}\left[\zeta^{2} \Phi^{\prime}\left(\zeta^{2}+4 \sinh ^{2} \frac{u}{2}\right)+\frac{1}{2} \Phi\left(\zeta^{2}+4 \sinh ^{2} \frac{u}{2}\right)\right] \\
=\int_{-\infty}^{\infty} d \zeta\left\{\left(\frac{\zeta-2 i \cosh \frac{u}{2}}{\zeta-2 i \cosh \frac{u}{2}}\right)^{\frac{m}{2}} \zeta^{2} \Phi^{\prime}\left(\zeta^{2}+4 \sinh ^{2} \frac{u}{2}\right)\right. \\
\left.-\frac{1}{2} \zeta \frac{d}{d \zeta}\left[\Phi\left(\zeta^{2}+4 \sinh ^{2} \frac{u}{2}\right)\left(\frac{\zeta-2 i \cosh \frac{u}{2}}{\zeta+2 i \cosh \frac{u}{2}}\right)^{\frac{m}{2}}\right]\right\} \\
=-i m \cosh \frac{u}{2} \int_{-\infty}^{\infty} d \zeta\left(\frac{\zeta-2 i \cosh \frac{u}{2}}{\zeta+2 i \cosh \frac{u}{2}}\right)^{\frac{m}{2}} \frac{\zeta \Phi\left(\zeta^{2}+4 \sinh ^{2} \frac{u}{2}\right)}{\zeta^{2}+4 \cosh ^{2} \frac{u}{2}} .
\end{gathered}
$$

Thus we get for $A(\gamma)$

$$
A(\gamma)=\frac{\ln N_{\gamma_{0}}}{4 \sinh ^{2} \frac{u}{2}} \int_{-\infty}^{\infty} d \zeta\left(\frac{\zeta-2 i \cosh \frac{u}{2}}{\zeta+2 i \cosh \frac{u}{2}}\right)^{\frac{m}{2}}
$$

$\times\left[4 \sinh ^{2} \frac{u}{2} \Phi^{\prime}\left(\zeta^{2}+4 \sinh ^{2} \frac{u}{2}\right)+\left(1-\lambda_{\gamma} \cosh \frac{u}{2}\right) \Psi\left(\zeta^{2}+4 \sinh ^{2} \frac{u}{2}\right)\right.$

$=\frac{\ln N_{\gamma_{0}}}{4 \sinh \frac{u}{2}} \int_{0}^{\infty} \frac{d \zeta}{\left(\zeta^{2}+4 \sinh ^{2} \frac{u}{2}\right)^{\frac{m}{2}}}$

$$
\left.+\frac{i m \zeta}{\zeta^{2}+4 \cosh ^{2} \frac{u}{2}}\left(\chi_{\gamma}-\cosh \frac{u}{2}\right) \Phi\left(\zeta^{2}+4 \sinh ^{2} \frac{u}{2}\right)\right] .
$$

$\times\left\{\left[4 \sinh ^{2} \frac{u}{2} \Phi^{\prime}\left(\zeta^{2}+4 \sinh ^{2} \frac{u}{2}\right) \div\left(1-\lambda_{2} \cosh \frac{u}{2}\right) \Psi\left(\zeta^{2}+4 \sinh ^{2} \frac{u}{2}\right)\right]\left(\alpha_{+}^{m}+\alpha_{--}^{m}\right)\right.$

$$
\left.+\frac{i m \zeta}{\zeta^{2}+4 \cosh ^{2} \frac{u}{2}}\left(\lambda_{2}-\cosh \frac{u}{2}\right) \Phi\left(\zeta^{2}+4 \sinh ^{2} \frac{u}{2}\right)\left(\alpha_{+}^{m}-\alpha_{-}^{m}\right)\right\}
$$

(Substitution $x=\zeta^{2}+4 \sinh ^{2} \frac{u}{2} ; d x=2 \zeta d \zeta=2 \sqrt{x-4 \sinh ^{2} \frac{u}{2}} d \zeta$ )

$=\frac{\ln N_{\gamma_{0}}}{8 \sinh \frac{u}{2}} \int_{4 \sinh ^{2} \frac{u}{2}}^{\infty} \frac{d x}{\left(x+4 \sinh ^{2} \frac{u}{2}\right)^{\frac{m}{2}}}$

$\times\left[\frac{\left(1-\lambda_{\gamma} \cosh \frac{u}{2}\right) \Psi(x)+4 \sinh ^{2} \frac{u}{2} \Phi^{\prime}(x)}{\sqrt{x-4 \sinh ^{2} \frac{u}{2}}}\left(\alpha_{+}^{m}+\alpha_{-}^{m}\right)+i m\left(\chi_{\gamma}-\cosh \frac{u}{2}\right) \Phi(x) \frac{\alpha_{+}^{m}-\alpha_{-}^{m}}{x+4}\right]$

[and finally by Eqs.(37) and (38)]

$$
A(\gamma)=\frac{\ln N_{\gamma_{0}}}{N_{\gamma} \frac{1}{2}-N_{\gamma}-\frac{1}{2}}\left[g(u)+g(-u)-\chi_{\gamma}\left(g(u) e^{-\frac{u}{2}}+g(-u) e^{\frac{u}{2}}\right)\right] .
$$


This is the result of Ref.[10]. Therefore the supertrace formula reads:

$$
\begin{aligned}
& \operatorname{sir} L=i^{m}(g-1) \pi \Phi_{m}(0) \\
& \quad+\sum_{\{\gamma\}_{p}} \frac{\chi_{\gamma}{ }^{m} \ln N_{\gamma_{0}}}{N_{\gamma}^{\frac{1}{2}}-N_{\gamma}{ }^{-\frac{1}{2}}}\left[g(u)+g(-u)-\chi_{\gamma}\left(g(u) e^{-\frac{u}{2}}+g(-u) e^{\frac{u}{2}}\right)\right]
\end{aligned}
$$

where $u=\ln N_{\gamma}=l_{\gamma}$ and $g(u)$ is given by

$$
g(u)=\frac{1}{2 \pi} \int_{-\infty}^{\infty} d p e^{-i u p} h\left(i p+\frac{m+1}{2}\right) .
$$

Furthermore in $\Phi(x)$ an index $m$ is added to denote the dependence on $m$. Our final task is to eliminate $\Phi_{m}(0)$

Let us first consider $m=0$. By Eqqs.(39):

$$
g(u)-g(-u)=2 \sinh \frac{u}{2} \cosh \frac{u}{2} \int_{4 \sinh ^{2} \frac{u}{2}}^{\infty} \frac{\Phi_{0}^{\prime}(x) d x}{\sqrt{x-4 \sinh ^{2} \frac{u}{2}}} .
$$

Let us denote $\left(w=4 \sinh ^{2} \frac{u}{2}\right)$ :

$$
Q_{0}(w)=\frac{1}{\sinh u}[g(u)-g(-u)]=\int_{w}^{\infty} \frac{\Phi_{0}^{\prime}(x)}{\sqrt{x-w}} d x .
$$

Let us consider the integral

$$
\begin{aligned}
& -\frac{1}{\pi} \int_{x}^{\infty} \frac{d w}{\sqrt{w-x}} Q_{0}(w)=\int_{x}^{\infty} \frac{d w}{\sqrt{w-x}} \int_{w^{\prime}}^{\infty} \frac{\Phi_{0}^{\prime}(y)}{\sqrt{y-w}} d y \\
= & -\frac{1}{\pi} \int_{x}^{\infty} d y \Phi_{0}^{\prime}(y) \int_{w^{\prime}}^{y} d w\left(w^{\prime}-x\right)^{-\frac{1}{2}}\left(x-w^{\prime}\right)^{-\frac{1}{2}} \\
= & -\frac{1}{\pi} \frac{1}{2} B\left(\frac{1}{2}, \frac{3}{2}\right) \int_{x}^{\infty} d y \Phi_{0}^{\prime}(y)=\Phi_{0}(x),
\end{aligned}
$$

where in the last step the integral $[33, \mathrm{p} .285]$ :

$$
\int_{a}^{b}(x-a)^{\mu-1}(b-x)^{\nu-1} d x=(b-a)^{\nu+\mu-1} B(\nu, \mu)
$$

was used and $B(x, y)=\Gamma(x) \Gamma(y) / \Gamma(x+y)$ is the Beta function. Thus the inversion formula reads

$$
\Phi_{0}(x)=-\frac{1}{\pi} \int_{x}^{\infty} \frac{d w}{\sqrt{w-x}} Q_{0}(w) .
$$

Therefore:

$$
\begin{aligned}
& \Phi_{0}(0)=-\frac{1}{\pi} \int_{0}^{\infty} \frac{d u}{\sqrt{w}} Q_{0}(w)=-\frac{1}{\pi} \int_{0}^{\infty} \frac{d u}{\sinh \frac{u}{2}}[g(u)-g(-u)] \\
= & -\frac{1}{2 \pi^{2}} \int_{0}^{\infty} \frac{d u}{\sinh \frac{u}{2}} \int_{-\infty}^{\infty} d p\left[\varepsilon^{-i u p} h\left(i p+\frac{1}{2}\right)-c^{i u p} h\left(i p+\frac{1}{2}\right)\right] \\
= & \frac{i}{\pi^{2}} \int_{-\infty}^{\infty} d p h\left(i p+\frac{1}{2}\right) \int_{0}^{\infty} \frac{\sin u p}{\sinh \frac{u}{2}} d u \\
\because & \frac{i}{\pi} \int_{-\infty}^{\infty} h\left(i p+\frac{1}{2}\right) \tanh \pi p d p
\end{aligned}
$$

where the integral $[33$, p.503]:

$$
\int_{0}^{\infty} \frac{\sin (a x)}{\sinh (b x)} d x=\frac{\pi}{2 b} \tanh \frac{a \pi}{2 b}
$$

has been used; therefore finally ${ }^{3}$

$$
A_{0}^{(0)}=i(g-1) \int_{-\infty}^{\infty} h\left(i p+\frac{1}{2}\right) \tanh \pi p d p
$$

It is possible to construct the inversion formulas for, e.g. the $m=1$ and $m=$ 2 cases by starting from Eq.(39). But this is rather tedious and cannot be easily generalized to all $m \in \mathbf{Z}$. Therefore I must develop a systematic approach to invert Eq.(39), i.e. to express $\Phi_{m}(x)$ by an integal (or integrals) over $g(u)-g(-u)$. The general inversion formula must then be evaluated for $\Phi_{m}(0)$.

Let us consider Eq.(39) by reinserting the variable $\xi=\sqrt{x-4 \sinh ^{2} \frac{u}{2}}$;

$$
\begin{aligned}
g(u)-g(-u) & =\frac{1}{2} \sinh \frac{u}{2} \int_{-\infty}^{\infty} d \xi\left(\frac{\xi-2 i \cosh \frac{u}{2}}{\xi+2 i \cosh \frac{u}{2}}\right)^{\frac{m}{2}} \\
& \times\left[4 \cosh \frac{u}{2} \Phi_{m}^{\prime}\left(\xi^{2}+4 \sinh ^{2} \frac{u}{2}\right)-\frac{i m \xi}{\xi^{2}+4 \cosh ^{2} \frac{u}{2}} \Phi_{m}\left(\xi^{2}+4 \sinh ^{2} \frac{u}{2}\right)\right] .
\end{aligned}
$$

Let be $m \neq 0$. Performing a partial integration in the second term, where it is assumed that all the relevant terms are sufficiently decreasing at $\infty$ :

$$
\begin{aligned}
& \int_{-\infty}^{\infty} d \xi\left[\left(\frac{\xi-2 i \cosh \frac{u}{2}}{\xi+2 i \cosh \frac{u}{2}}\right)^{\frac{m}{2}} \frac{1}{\xi^{2}+4 \cosh ^{2} \frac{u}{2}}\right] \cdot\left[\xi \Phi_{m}\left(\xi^{2}+4 \sinh ^{2} \frac{u}{2}\right)\right] \\
= & -\frac{1}{2 i m \cosh \frac{u}{2}} \int_{-\infty}^{\infty} d \xi\left(\frac{\xi-2 i \cosh \frac{u}{2}}{\xi+2 i \cosh \frac{u}{2}}\right)^{\frac{m}{2}}\left[\Phi_{m}\left(\xi^{2}+4 \sinh ^{2} \frac{u}{2}\right)+2 \xi^{2} \Phi_{m}^{\prime}\left(\xi^{2}+4 \sinh ^{2} \frac{u}{2}\right)\right] .
\end{aligned}
$$

With the abbreviation $u=4 \sinh ^{2} \frac{u}{2}$ this gives in Eq.(53):

$$
\begin{aligned}
& g(u)-g(-u) \\
= & \frac{1}{2} i^{m} \tanh \frac{u}{2} \int_{-\infty}^{\infty} d \xi\left(\frac{\sqrt{w+4}+i \xi}{\sqrt{w+4}-i \xi}\right)^{\frac{m}{2}}\left[\left(w+\xi^{2}+4\right) \Phi_{m}^{\prime}\left(w+\xi^{2}\right)+\frac{1}{2} \Phi_{m}\left(w+\xi^{2}\right)\right] . \quad \text { (55) }
\end{aligned}
$$

Let us define $Q$ must not be confused with $Q_{0}$ in Eq.(4i).

$$
\begin{aligned}
Q(w) & =2 \operatorname{coth} \frac{u}{2}[g(u)-g(-u)] \\
\dot{\Phi}_{m}(x) & =i^{m}\left[(x+4) \Phi_{m}^{\prime}(x)+\frac{1}{2} \Phi_{m}(x)\right]
\end{aligned}
$$

and get the integral relation $\left(w=4 \sinh ^{2} \frac{u}{2} \geq 0\right)$ :

$$
Q\left(u^{\prime}\right)=\int_{-\infty}^{\infty} d \xi\left(\frac{\sqrt{w+4}-i \xi}{\sqrt{u+4}-i \xi}\right)^{\frac{m}{2}} \tilde{\Phi}_{m}\left(w+\xi^{2}\right) .
$$

In Ref. 10 a factor of two is missiug. 
For this integral relation I can apply an inversion formula given by Hejhal [49. pp.454] which yields for $\bar{\Phi}$ :

$$
\tilde{\Phi}_{m}(x)=-\frac{1}{\pi} \int_{-\infty}^{\infty} Q^{\prime}\left(x+t^{2}\right)\left(\frac{\sqrt{x+4+t^{2}}-t}{\sqrt{x+4+t^{2}}+t}\right)^{\frac{m}{2}} d t
$$

Reinserting $\tilde{\Phi}$ I get a differential equation for $\Phi$ :

$$
\Phi_{m}^{\prime}(x)+\frac{1}{2(x+4)} \Phi_{m}(x)=\frac{-1}{i^{m} \pi(x+4)} \int_{-\infty}^{\infty} Q^{\prime}\left(x+t^{2}\right)\left(\frac{\sqrt{x+4+t^{2}}-t}{\sqrt{x+4+t^{2}}+t}\right)^{\frac{m}{2}} d t \quad(59)
$$

which can be easily solved to give the inversion formula for $\Phi$ :

$$
i^{m} \Phi_{m}(x)=\frac{1}{\pi \sqrt{x+4}} \int_{x}^{\infty} \frac{d y}{\sqrt{y+4}} \int_{-\infty}^{\infty} Q^{\prime}\left(y+t^{2}\right)\left(\frac{\sqrt{y+4+t^{2}}-t}{\sqrt{y+4+t^{2}}+t}\right)^{\frac{m}{2}} d t .
$$

This is the main result of this section.

Note: 1) The integration constant in Eq.(60) is given by $\Phi_{m}(\propto)=0$.

2) The inversion formula is valid for $m \in \mathbf{Z}$ [see below $\mathbf{E} q .(61)$ for $m=0$ ].

To get some confidence in the inversion formula let us consider $\mathrm{Eq}_{\mathrm{q}}(60)$ for some specific values of $m$.

1) $m=0$ :

$$
\Phi_{0}(x)=\frac{1}{\pi \sqrt{x+4}} \int_{x}^{\infty} \frac{d y}{\sqrt{y+4}} \int_{-\infty}^{\infty} Q^{\prime}\left(y \div t^{2}\right) d t
$$

$\left(u=y^{2}+t\right)$

$$
=\frac{1}{\pi \sqrt{x+4}} \int_{x}^{\infty} \frac{d y}{\sqrt{y+4}} \int_{y}^{\infty} \frac{Q^{\prime}\left(w^{\prime}\right)}{\sqrt{u^{\prime}-y}} d w
$$

(Rearrangement of integrations)

$$
=\frac{1}{\pi \sqrt{x+4}} \int_{x}^{\infty} d w Q^{\prime}(w) \int_{x}^{w} \frac{d y}{\sqrt{y+4} \sqrt{w-y}}
$$

[Elementary integral: $\left.\int \frac{d x}{\sqrt{(a x+b)(c x+d)}}-\frac{2}{\sqrt{-a c}} \arctan \sqrt{-\frac{x+\frac{b}{a}}{x+\frac{d}{c}}}(a c<0)\right]$

$$
=\frac{2}{\pi \sqrt{x+4}} \int_{x}^{\infty} Q^{\prime}(w) \arctan \sqrt{\frac{w-x}{x+4}} d w
$$

(Partial integration)

$$
\begin{aligned}
& =-\frac{1}{\pi} \int_{\pi}^{\infty} \frac{Q(w)}{(u+4) \sqrt{u-x}} d u \\
= & \left.4 \sinh ^{2} \frac{u}{2}\right) \\
& =-\frac{1}{\pi} \int_{2 \operatorname{arsinh} \frac{\sqrt{x}}{2}}^{\infty} \frac{g(u)-g(-u)}{\sqrt{4 \sinh ^{2} \frac{u}{2}-x}} d u .
\end{aligned}
$$$$
\left(u=4 \sinh ^{2} \frac{u}{2}\right)
$$

This is equivalent with Eq.(50) and shows that the inversion formula is also valid for $m=0$, i.e. the inversion formula is valid for all $m \in \mathbf{Z}$.
2) $m=1$ :

$$
\begin{aligned}
& i \Phi_{\mathrm{I}}(x)=\frac{1}{\pi \sqrt{x-4}} \int_{x}^{\infty} \frac{d y}{\sqrt{y+4}} \int_{-\infty}^{\infty} Q^{\prime}\left(y+t^{2}\right)\left(\frac{\sqrt{y+4+t^{2}}-t}{\sqrt{y+4+t^{2}}+t}\right)^{\frac{2}{2}} d t \\
& \quad=\frac{2}{\pi \sqrt{x+4}} \int_{x}^{\infty} \frac{d y}{y-4} \int_{0}^{\infty} Q^{\prime}\left(y+t^{2}\right) \sqrt{y+4+t^{2}} d t \\
& \left(w=y^{2}-t\right) \\
& =\frac{1}{\pi \sqrt{x+4}} \int_{x}^{\infty} \frac{d y}{y+4} \int_{y}^{\infty} Q^{\prime}\left(w^{\prime}\right) \sqrt{\frac{w+4}{w-y}} d u
\end{aligned}
$$

(Rearrangement of integrations)

$$
=\frac{1}{\pi \sqrt{x+4}} \int_{x}^{\infty} d w Q^{\prime}(w) \sqrt{u+4} \int_{x}^{w} \frac{d y}{(y+4) \sqrt{u-y}}
$$

(Set $z=y+4$ and elementary integral: $\int \frac{d x}{x \sqrt{a}-\bar{b}}=-\frac{2}{\sqrt{b}} \operatorname{artanh} \sqrt{\frac{a x+b}{b}}$ )

$$
=\frac{2}{\pi \sqrt{x+4}} \int_{x}^{\infty} Q^{\prime}\left(u^{\prime}\right) \operatorname{artanh} \sqrt{\frac{u^{\prime}-\bar{s}}{u+4}} d u
$$

(Partial integration)

$$
=-\frac{1}{\pi \sqrt{x+4}} \int_{x}^{\infty} \frac{Q(w)}{\sqrt{(w-x)(w+4)}} d w
$$

$\left(w=4 \sinh ^{2} \frac{u}{2}\right)$

$$
=-\frac{4}{\pi \sqrt{x+4}} \int_{2 \mathrm{ar} \sinh \frac{\sqrt{x}}{2}}^{\infty} \frac{g(u)-g(\cdots u)}{\sqrt{4 \sinh ^{2} \frac{u}{2}-s}} \cosh \frac{u}{2} d u .
$$

In particular for $x=0$ :

$$
\begin{aligned}
& i \Phi_{1}(0)=-\frac{1}{\pi} \int_{0}^{\infty} \operatorname{coth} \frac{u}{2}[g(u)-g(-u) d u \\
& =\frac{i}{\pi^{2}} \int_{-\infty}^{\infty} d p h_{1}\left(i p+\frac{1}{2}\right) \int_{0}^{\infty} \operatorname{coth} \frac{u}{2} \sin u p d u \\
& \quad=\frac{i}{\pi} \int_{-\infty}^{\infty} h_{1}\left(i p+\frac{1}{2}\right) \operatorname{coth} \pi p d p
\end{aligned}
$$

where in the last step the integral $[33$, p. 504

$$
\int_{0}^{\infty} \sin a x \frac{\cosh \beta x}{\sinh \gamma x} d x=\frac{\pi}{2 \gamma} \frac{\sinh \frac{\pi a}{\gamma}}{\cosh \frac{\pi a}{\gamma}+\cos \frac{\pi \beta}{\gamma}}
$$

was used. This gives finally for $A_{0}^{(1)}$ by Eq. (23)

$$
A_{0}^{(1)}=i(g-1) \int_{-\infty}^{\infty} \operatorname{coth} \pi p h(i p+1) d p .
$$


3) $m=2$ :

$$
\begin{aligned}
& i^{2} \Phi_{2}(x)=\frac{1}{\pi \sqrt{x+4}} \int_{x}^{\infty} \frac{d y}{\sqrt{y+4}} \int_{-\infty}^{\infty} Q^{\prime}\left(y+t^{2}\right)\left(\frac{\sqrt{y+4+t^{2}}-t}{\sqrt{y+4+t^{2}}+t}\right) d t \\
& =\frac{2}{\pi \sqrt{x+4}} \int_{0}^{\infty} \frac{d y}{\sqrt{y+4}} \int_{0}^{\infty} Q^{\prime}\left(y+t^{2}\right) d t \\
& \quad+\frac{4}{\pi \sqrt{x+4}} \int_{0}^{\infty} \frac{d y}{\sqrt{y+4}} \int_{0}^{\infty} t^{2} Q^{\prime}\left(y+t^{2}\right) d t . \quad(66)
\end{aligned}
$$

The first integral is up to a factor Eq.(61). For the second I get:

$$
\begin{aligned}
& \frac{4}{\pi \sqrt{x+4}} \int_{0}^{\infty} \frac{d y}{\sqrt{y+4^{3}}} \int_{0}^{\infty} t^{2} Q^{\prime}\left(y+t^{2}\right) d t \\
& =\frac{2}{\pi \sqrt{x+4}} \int_{x}^{\infty} \frac{d y}{(y+4)^{\frac{3}{2}}} \int_{0}^{\infty} t\left[\frac{d}{d t} Q\left(y+t^{2}\right)\right] d t \quad \text { (Partial integration) } \\
& =-\frac{2}{\pi \sqrt{x+4}} \int_{x}^{\infty} \frac{d y}{(y+4)^{\frac{3}{2}}} \int_{0}^{\infty} Q\left(y+t^{2}\right) d t \quad\left(w=y+t^{2}\right. \text { and rearrangement) } \\
& \quad=-\frac{1}{\pi \sqrt{x+4}} d w Q(w) \int_{x}^{w} \frac{d y}{\sqrt{w-y} \sqrt{y+4}} \\
& \text { (Elementary integral: } \left.\int \frac{d x}{\sqrt{a x+b} \sqrt{c x+d^{3}}}=\frac{2}{a d-b c} \sqrt{\frac{a x+b}{c x+d}}\right) \quad\left(w=4 \sinh ^{2} \frac{u}{2}\right) \\
& \quad=-\frac{2}{\pi(x+4)} \int_{x}^{\infty} \frac{\sqrt{w-x}}{w+4} Q(w) d w \\
& =-\frac{4}{\pi(x+4)} \int_{2 \operatorname{arsinh} \frac{\sqrt{x}}{2}}^{\infty}[g(u)-g(-u)] \sqrt{4 \sinh ^{2} \frac{u}{2}-x} d u .
\end{aligned}
$$

This gives for $m=2$ the inversion formula

$$
\begin{aligned}
i^{2} \Phi_{2}(x)=-\frac{1}{\pi} \int_{2 \operatorname{arsinh} \frac{\sqrt{x}}{2}}^{\infty} & \frac{g(u)-g(-u)}{\sqrt{4 \sinh ^{2} \frac{u}{2}-x}} d u . \\
& -\frac{4}{\pi(x+4)} \int_{2 \operatorname{arsinh} \frac{\sqrt{x}}{2}}^{\infty}[g(u)-g(-u)] \sqrt{4 \sinh ^{2} \frac{u}{2}-x} d u .
\end{aligned}
$$

In particular for $x=0$

$$
i^{2} \Phi_{2}(0)=\frac{i}{\pi} \int_{-\infty}^{\infty} h_{2}\left(i p+\frac{1}{2}\right) \tanh \pi p d p-\frac{2}{\pi} \int_{-\infty}^{\infty} g(u) \sinh \frac{u}{2} d u
$$

and therefore finally

$$
A_{0}^{(2)}=i(g-1) \int_{-\infty}^{\infty} h_{2}\left(i p+\frac{1}{2}\right) \tanh \pi p d p+(1-g)\left[h_{2}(1)-h_{2}(0)\right] .
$$

4) $m=3$ : Similarly as for $m=2$ it is straightforward to show that

$$
\begin{gathered}
i^{3} \Phi_{3}(0)=\frac{i}{\pi} \int_{-\infty}^{\infty} h_{3}\left(i p+\frac{1}{2}\right) \operatorname{coth} \pi p d p-\frac{2}{\pi} \int_{-\infty}^{\infty} g(u) \sinh u d u \\
f_{11}^{(3)}-i(g-1) \int_{-\infty}^{\infty} h_{3}\left(i p+\frac{1}{2}\right) \operatorname{coth} \pi p d p-(1-g)\left[h_{3}\left(\frac{3}{2}\right)-h_{3}\left(-\frac{1}{2}\right)\right.
\end{gathered}
$$

Let us for a moment turn to test functions $h$ for the operator $\hat{Q}$, i.e.let us consider the functions $h(s) \equiv h_{0}(s)$. By Eqs.(34) relating $h$ and $g, \hat{g}$ and $\hat{Q}$ do not depend on $m$ ("hatted" quantities belonging to $\widehat{\Pi}_{m}$ ). The equations for $\hat{\Phi}_{m}$ $(m=0,1,2,3)$ suggest the following general structure for $\hat{\Phi}_{m}$

$i^{m} \hat{\Phi}_{m}(0)=\frac{i}{\pi} \int_{-\infty}^{\infty} h\left(i p+\frac{1}{2}\right) \tanh \pi p d p$

$$
-\frac{2}{\pi} \sum_{k=1}^{m / 2} \int_{-\infty}^{\infty} \hat{g}(u) \sinh \left(k-\frac{1}{2}\right) u d u \quad \text { (m even) }
$$

$i^{m} \hat{\Phi}_{m}(0)=\frac{i}{\pi} \int_{-\infty}^{\infty} h\left(i p+\frac{1}{2}\right) \operatorname{coth} \pi p d p$

$$
-\frac{2}{\pi} \sum_{k=1}^{(m-1) / 2} \int_{-\infty}^{\infty} \hat{g}(u) \sinh k u d u \quad(m \text { odd }) .
$$

In particularly, it remains to show that for all $m$ (even and odd):

$$
i^{m+2} \hat{\Phi}_{m+2}(0)-i^{m} \hat{\Phi}_{m}(0)=-\frac{2}{\pi} \int_{-\infty}^{\infty} \hat{g}(u) \sinh \frac{m+1}{2} d u .
$$

Having proved Eq.(74) once, one can go back to the operator $\square_{m}$ and all related quantities. Equation (74) is now proved by induction for $m \rightarrow m+2$.

1) Since each step forward is by two units in the induction we have to distinguish between the even and odd cases. Eqs.(61) and (69), respectively (63) and (71) show that Eq.(74) is correct for $m=0$ and $m=1$, respectively.

2) Let us consider Eq. $(74)$ and insert for $i^{m} \hat{\Phi}_{m}(0)$ and $i^{m+2} \hat{\Phi}_{m+2}(0)$ :

$$
\begin{aligned}
& i^{m+2} \hat{\Phi}_{m+2}(0)-i^{m} \hat{\Phi}_{m}(0)= \frac{1}{2 \pi} \int_{x}^{\infty} \frac{d y}{\sqrt{y+4}} \hat{Q}^{\prime}\left(y+t^{2}\right) \\
& \times {\left[\left(\frac{\sqrt{y+4+t^{2}}-t}{\sqrt{y+4+t^{2}}+t}\right)^{\frac{\frac{m+2}{2}}{2}}-\left(\frac{\sqrt{y+4+t^{2}}-t}{\sqrt{y+4+t^{2}}+t}\right)^{\frac{m}{2}}\right] } \\
&=-\frac{1}{\pi} \int_{0}^{\infty} \frac{d y}{(y+4)^{\frac{m+5}{2}}} \int_{-\infty}^{\infty} t \dot{Q}^{\prime}\left(y+t^{2}\right)\left(\sqrt{y+4+t^{2}}-t\right)^{m+1} d t
\end{aligned}
$$

(Partial integration)

$$
\begin{aligned}
& =-\frac{m+1}{2 \pi} \int_{0}^{\infty} \frac{d y}{(y+4)^{\frac{m+3}{2}}} \int_{-\infty}^{\infty} \hat{Q}\left(y+t^{2}\right)-\frac{\left(\sqrt{y+4+t^{2}}-t\right)^{m+1}}{\sqrt{y+4+t^{2}}} d t \\
& =-\frac{m+1}{2 \pi} \int_{0}^{\infty} \frac{d y}{(y+4)^{\frac{m-3}{2}}} \\
& \quad \quad \times \int_{0}^{\infty} \frac{\hat{Q}\left(y+t^{2}\right)}{\sqrt{y+4+t^{2}}}\left[\left(\sqrt{y+4+t^{2}}-t\right)^{m+1}+\left(\sqrt{y+4+t^{2}}+t\right)^{m+1}\right]
\end{aligned}
$$

$\left(w=4 \sinh ^{2} \frac{u}{2}\right)$

$$
\begin{aligned}
= & -\frac{m+1}{4 \pi} \int_{0}^{\infty} \frac{d y}{(y \div 4)^{\frac{m+5}{2}}} \\
& \times \int_{y}^{\infty} \frac{Q(w)}{\sqrt{w} w-4} \sqrt{v^{w}-y}\left[(\sqrt{w+4}-\sqrt{w-y})^{m+1}+(\sqrt{w-4}+\sqrt{w-y})^{m \cdots 1}\right] .
\end{aligned}
$$


Again with a rearrangement of integrations:

$$
\begin{aligned}
i^{m+2} \hat{\Phi}_{m+2}(0) & -i^{m} \hat{\Phi}_{m}(0)=-\frac{m+1}{4 \pi} \int_{0}^{\infty} d w \frac{\hat{Q}(w)}{\sqrt{w+4}} \\
& \times \int_{0}^{w} \frac{d y}{\sqrt{w-y}}\left[\frac{(\sqrt{w+4}-\sqrt{w-y})^{m+1}}{(y+4)^{\frac{m+3}{2}}}+\frac{\left(\sqrt{w}+4+\sqrt{w^{-}-y}\right)^{m+1}}{(y+4)^{\frac{m+s}{2}}}\right]
\end{aligned}
$$

[Substitution $t^{2}=1-(y+4) /(w+4)$ ]

$$
=-\frac{m+1}{2 \pi} \int_{0}^{\infty} d w \frac{\hat{Q}(w)}{w+4} \int_{0}^{\sqrt{\frac{w}{u+4}}}\left[\frac{(1-t)^{\frac{m-1}{2}}}{(1+t)^{\frac{m+3}{2}}}+\frac{(1+t)^{\frac{m-1}{2}}}{(1-t)^{\frac{m+3}{2}}}\right] d t .
$$

Let us consider the $t$-integration and replace $m \rightarrow m+2$

$(m+3) \int_{0}^{\sqrt{\frac{w^{m}}{w+4}}}\left[\frac{(1-t)^{\frac{m+1}{2}}}{(1+t)^{\frac{m+5}{2}}}+\frac{(1+t)^{\frac{m+1}{2}}}{(1-t)^{\frac{m+5}{2}}}\right] d t$

(Partial integration:)

$$
\begin{gathered}
=\underbrace{-\left.2\left[\frac{(1-t)^{\frac{m+1}{2}}}{(1+t)^{\frac{m+3}{2}}}-\frac{(1+t)^{\frac{m+1}{2}}}{(1-t)^{\frac{m+3}{2}}}\right]\right|_{0} ^{\sqrt{\frac{w}{w+4}}}}_{=4 \cosh \frac{u}{2} \sinh \frac{m+2}{2} u} \\
\quad-(m+1) \int_{0}^{\sqrt{\left.\frac{w}{w}{ }^{2} \frac{u}{2} \text { reinserted }\right)}} \\
\left.\quad \frac{(1-t)^{\frac{m-1}{2}}}{(1+t)^{\frac{m+3}{2}}}+\frac{(1+t)^{\frac{m-1}{2}}}{(1-t)^{\frac{m+3}{2}}}\right] d t .
\end{gathered}
$$

Thus repeating the calculations of Eq. (75) for $m \rightarrow m+2$ and taking into account the result of Eq.(76) yield together with $w=4 \sinh ^{2} \frac{u}{2}$ and Eq.(74):

$$
\begin{aligned}
i^{m+4} & \hat{\Phi}_{m+4}(0)-i^{m+2} \hat{\Phi}_{m+2}(0) \\
& =-\frac{8}{\pi} \int_{0}^{\infty}[\hat{g}(u)-\hat{g}(-u)] \cosh \frac{u}{2} \sinh \frac{m+2}{2} d u-\left[i^{m+2} \hat{\Phi}_{m+2}(0)-i^{m} \hat{\Phi}_{m}(0)\right] \\
& =-\frac{2}{\pi} \int_{-\infty}^{\infty} \hat{g}(u) \sinh \frac{m+3}{2} d u .
\end{aligned}
$$

This proves the induction!

I summarize. I have formulated the Selberg Supertrace formula on super Riemannia surfaces for operator valued functions of the Laplace-Dirac operator $\square_{m}$. Let $h$ be a testfunction with the properties (following Baranov et al. [10]):

i) $h\left(\frac{1}{2}+i p\right) \in C^{\infty}(\mathbf{R})$

ii) $h\left(\frac{1}{2}+i p\right)$ need not be an even function in $p$

iii) $h\left(\frac{1}{2}+i p\right) \propto O\left(\frac{1}{p^{2}}\right)(p \rightarrow \pm \infty)$.

iv) $h\left(\frac{1}{2}+i p\right)$ is holomorphic in the strip $|\operatorname{Im}(p)| \leq 1+\frac{m}{2}+\epsilon, \epsilon=0$ to guarantee absolute convergence in the sums of Eq.(82) below (see [49] p.30]).

Its Fourier transform $g$ is given by:

$$
g(u)=\frac{1}{2 \pi} \int_{-\infty}^{\infty} d p e^{-i u p} h\left(i p+\frac{m+1}{2}\right) .
$$

The term $A_{0}^{(m)}$ corresponding to the identity transformation reads

$$
\begin{aligned}
& A_{0}^{(m)}=i(g-1) \int_{-\infty}^{\infty} h\left(i p+\frac{m+1}{2}\right) \tanh \pi p d p \\
& \quad-(1-g) \sum_{k=1}^{m / 2}\left[h\left(\frac{m}{2}+k\right)-h\left(\frac{m}{2}-k-1\right)\right] \quad \text { (m even) } \\
& A_{0}^{(m)}=i(g-1) \int_{-\infty}^{\infty} h\left(i p+\frac{m+1}{2}\right) \operatorname{coth} \pi p d p \\
& +(1-g) \sum_{k=1}^{(m-1) / 2}\left[h\left(\frac{m+1}{2}+k\right)-h\left(\frac{m-1}{2}-k\right)\right] \quad(m \text { odd }) .
\end{aligned}
$$

The last two equations can be combined and stated in a compact form yielding ${ }^{1}$

$$
A_{0}^{(m)}=(1-g) \int_{0}^{\infty} \frac{g(u)-g(-u)}{\sinh \frac{u}{2}} T_{m}\left(\cosh \frac{u}{2}\right) d u, \quad(m \in \mathbf{Z}),
$$

where $T_{m}\left(\cosh \frac{u}{2}\right)=\cosh \frac{m}{2} u$ denotes the $m^{\text {th }}$ Chebyshev-polynomial in $\cosh \frac{u}{2}$. Thus the supertrace formula reads $\left(l_{\gamma}\right.$ primitive geodesic):

$$
\begin{aligned}
& \sum_{n=0}^{\infty}\left[h_{m}\left(p_{n}^{B}\right)-h_{m}\left(p_{n}^{F}\right)\right]=(1-g) \int_{0}^{\infty} \frac{g(u)-g(-u)}{\sinh \frac{u}{2}} \cosh \frac{m}{2} d u \\
+ & \sum_{\{\gamma\}_{p}} \sum_{k=1}^{\infty} \frac{l_{\gamma} \chi_{\gamma}{ }^{k m}}{\epsilon^{\frac{k l_{2}}{2}}-\epsilon^{-\frac{k l_{2}}{2}}}\left[g\left(k l_{\gamma}\right)+g\left(-k l_{\gamma}\right)-x_{\gamma}{ }^{k}\left(g\left(k l_{\gamma}\right) e^{-\frac{k l_{\gamma}}{2}}+g\left(-k l_{\gamma}\right) \epsilon^{\frac{k l_{\gamma}}{2}}\right)\right]
\end{aligned}
$$

Equation (82) completes the work of Refs.[10,11] by explicit statement of the inversion formula (60) and the $A_{0}^{(m)}$-term, respectively.

\footnotetext{
' Note the similarity with the $A_{0, S c i b c r g}^{i m 1}$ term in $\mathbf{E}_{\mathbf{q}}$ (1.25).
} 


\section{Analytic Properties of the Selberg Super Zeta-Functions}

\section{The Selberg Zeta-Function $Z$}

The Selberg zeta-functions were originally introduced by Selberg [82] in order to study spectra of Laplacians on compact Riemann surfaces of genus $g$. The Selberg zeta-function is defined by

$$
Z(s):=\prod_{\{\gamma\}_{p}} \prod_{k=0}^{\infty}\left[1-e^{-(s+k) l_{\gamma}}\right], \quad(\operatorname{Re}(s)>1)
$$

Here $l_{\gamma}$ is the length of a primitive $\gamma \in \Gamma$ and $\{\gamma\}_{p}$ denotes primitive conjugacy classes. The analytic properties of $Z(s)$ can be studied by the Selberg trace formula with the help of the regularized resolvent function $h(p)=1 /\left[p^{2}-\left(s-\frac{1}{2}\right)^{2}\right]-1 /\left[p^{2}-\left(\sigma-\frac{1}{2}\right)^{2}\right]$. This is an even function with the property $h(p) \rightarrow O\left(p^{-4}\right)(p \rightarrow \pm \infty)$. Inserting $h$ into the Selberg trace formula (1.22) one finds for $\operatorname{Re}(s)>1[49,84]$

$$
\frac{1}{2 s-1} \frac{Z^{\prime}(s)}{Z(s)}=B+\frac{1}{s(s-1)}+2(g-1)[\Psi(s)-\Psi(1)]+\sum_{n=1}^{\infty}\left[\frac{1}{E_{n}+s(s-1)}-\frac{1}{E_{n}}\right],(2)
$$

where the $E_{n}=\frac{1}{4}+p_{n}^{2}$ are the Eigenvalues of the Laplacian $-\Delta$ on the Poincare upper half-plane in a compact domain as already defined in chapter I and $B=\frac{1}{2} \frac{Z^{\prime \prime}(1)}{Z^{\prime}(1)}-1$. Thus, $Z(s)$ is an entire function of $s$ of order 2 and Eq.(2) extends meromorphically to all $s \in \mathbf{C}$. Furthermore one can state: $Z(s)$ has

- : at $s=-k(k \in \mathrm{N})$ zeros with multiplicity $(2 g-2)(2 k+1)$

- : at $s=0$ a zero with multiplicity $2 g-1$ and

- : at $s=1$ a zero of multiplicity one.

These zeros are called the "trivial" ones. Furthermore:

- : at $s=\frac{1}{2} \pm i p_{n}\left(p_{n} \in \mathbf{R}\right)$ "nontrivial" zeros with the same multiplicity as the corresponding Eigenvalue,

- : at $0<s<1$ "nontrivial" zeros are located which correspond to the so called "small Eigenvalues" with the same multiplicity as the corresponding Eigenvalue.

These Eigenvalues are the Eigenvalues of the Laplacian $-\Delta$ defined on compact domains on the Poincaré upper haf-plane with periodic boundary conditions for its $4 g$ boundaries. These compact domains correspond to a compact Riemann surface of genus $g$. Setting $s \rightarrow 1-s$ in $\mathrm{Eq}$.(2) and subtracting it from (2) one gets by direct integration the functional equation for the Selberg zeta-function

$$
Z(s)=Z(1-s) \exp \left[4 \pi(g-1) \int_{0}^{s-\frac{1}{2}} y \tan \pi y d y\right] .
$$

One could hope of finding an analytic continuation of $Z(s)$ in, say. $0 \leq \operatorname{Re}(s) \leq 1$, where it should be possible to determine on the critical line $\operatorname{Re}(s)=\frac{1}{2}$ the nontrivial zeros and therefore the Eigenvalues $E_{n}=\frac{1}{4}+p_{n}^{2}$ of the corresponding Laplacian (small Eigenvalues, of course, in a similar manner; for the regular oktagon $(g=2)$ it is has recently been shown, that there are no such Eigenvalues $[7 \mid)$. Unfortunately, up to now no such analytic continuation has been constructed.

There is an increasing amount of literature concerning the Selberg zeta-function. It has been of interest, because determinants of Laplacians can be expressed by combinations of the zeta-function and its derivatives. Define $D_{\Delta}(z)=\operatorname{det}^{\prime}(-\Delta+z)$, where the prime denotes the omission of zero modes. Then the Selberg zeta-function and $D_{\Delta}$ are connected by the relation $[84]$ :

$$
Z(s)=s(s-1) D_{\Delta}[s(s-1)]\left[(2 \pi)^{1-s} e^{C+s(s-1)} G(s) G(s+1)\right]^{2(g-1)},
$$

where $C=\frac{1}{4}-\ln \sqrt{2 \pi}-2 \zeta^{\prime}(-1)$ and $G(z)$ denotes the Barnes $G$-function defined by

$$
\begin{aligned}
G(z+1) & =(2 \pi)^{\frac{z}{2}} e^{-\frac{z}{4}-\frac{1+\gamma_{E}}{2} z^{2}} \prod_{n=1}^{\infty}\left[\left(1+\frac{z}{n}\right)^{n} e^{-z+\frac{z^{2}}{2 n}}\right] \\
& =(2 \pi)^{\frac{2}{2}} e^{-\frac{2}{4}-\frac{1+\gamma_{E}}{2} z^{2}} \prod_{n=1}^{\infty}\left[\frac{\Gamma(n)}{\Gamma(z+n)} e^{z \Psi(n)+\frac{1}{2} z^{2} \Psi^{\prime}(n)}\right]
\end{aligned}
$$

$\left[\Psi(z)=\Gamma^{\prime}(z) / \Gamma(z)\right.$ the digamma-function, $\gamma_{E}=-\Psi(1)$ Euler's constant $] . G(z)$ has the important properties $G(z+1)=\Gamma(z) G(z)$ and $G(1)=1$. By taking the limit $s \rightarrow 1$ in Eq.(4) one gets

$$
\operatorname{det}^{\prime}(-\Delta)=Z^{\prime}(1) e^{(g-1)\left[\ln 2 \pi+4 \zeta^{\prime}(-1)-\frac{1}{2}\right]},
$$

where $\zeta(z)$ denotes the Riemann zeta-function. The properties of the Selberg zeta function can also be used to show that the bosonic string theorie diverges - see [45] and end of chapter VI. Steiner [84] has computed the inportant relation

$$
\begin{aligned}
Z(s)=s(s-1) Z^{\prime}(1) e^{\gamma \Delta s(s-1)}\left[(2 \pi)^{1-s} e^{s(s-1)} G(s) G(s+1)\right]^{2(g-1)} & \\
& \times \prod_{n=1}^{\infty}\left(1+\frac{s(s-1)}{E_{n}}\right) \exp \left(-\frac{s(s-1)}{E_{n}}\right)
\end{aligned}
$$

where $\gamma_{\Delta}=2(g-1)_{E}-\frac{Z^{\prime \prime}(1)}{2 Z^{\prime}(1)}-1$. Equations $(4)$ and $(\bar{t})$ are connected by the formula

$$
D_{\Delta}(z)=Z^{\prime}(1) \epsilon^{\gamma_{\Delta} z-2(g-1) C} \prod_{n=1}^{\infty}\left(1+\frac{z}{E_{n}}\right) \exp \left(\cdots \frac{z}{E_{n}}\right) .
$$

From $\mathrm{E}_{\mathrm{l}} \cdot(7)$ one can deduce

$$
Z(s)=Z(s+1) \frac{s-1}{s+1} \epsilon^{-2 s \gamma_{\Delta}}\left[\frac{s c^{2 s} \Gamma^{2}(s)}{2 \pi}\right]^{2(1-g)} \prod_{n=1}^{\infty} \frac{E_{n}+s(s-1)}{E_{n}+s(s+1)} \exp \left(\frac{2 s}{E_{n}}\right) .
$$

Finally the functional relation (3) can be rewritten vielding

$$
Z(s)=Z(1-s)\left[(2 \pi)^{1-2 s} \frac{G(s) G(s+1)}{G(1-s) G(2 \cdots s)}\right]^{2(g-1)} .
$$


Introducing the function $R(s)=Z(s) / Z(s+1)$ one finds the functional relation for the function $R(s)$ which reads

$$
R(s) R(-s)=\frac{Z(s) Z(-s)}{Z(1+s) Z(1-s)}=(2 \sin \pi s)^{4(g-1)} .
$$

More information about $Z(s)$ can be found, e.g. in $[24,49,62,84]$.

The purpose of the following sections is to discuss the analytic properties of the Selberg super zeta-functions $Z_{0}$ and $Z_{1}$ (definitions see below). It turns out that it is possible to proceed in a fashion similar to that used for the ordinary Selberg zeta-function, however, with some restrictions. Thus the program is:

- : to determine the "trivial" zeros,

- : to determine the "nontrivial" zeros,

- : to find functional equations and,

- : to find functional equations combining the two Selberg super zeta-functions.

Since in the supertrace formula terms with and without the character $x$, appear, I have to choose appropriate test functions which separate this combination. It turns out that I must choose odd test functions which are allowed in the supertrace formula, in contrast to the ordinary trace formula. Similarities to, as well as differences from the original pure bosonic case will occur. I also derive functional relations and relations between the two possible zeta-functions. In a paper by Baranov and Schwarz [11], such a relation was already stated, unfortunately however, with a missing factor.

\section{The Selberg Super Zeta-Function $Z_{1}$}

The Selberg super zeta-functions are defined by

$$
Z_{q}(s):=\prod_{\{\gamma\}_{p}} \prod_{k=0}^{\infty}\left[1 \cdots \chi_{\gamma}{ }^{q} e^{-(v+k) l_{\gamma}}\right], \quad(\operatorname{Re}(s)>1),
$$

where $q$ can take on the values $q=0,1$, respectively. $\chi_{\gamma}$ describes the spin structure and $l_{\gamma}$ is the length of a primitive geodesic, as already defined. ${ }^{1}$ The $\gamma$ product is taken over all primitive conjugacy classes $\gamma \in \Gamma$. The Selberg super $R$-functions are defined by

$$
R_{q}(s):=\frac{Z_{q}(s)}{Z_{q}(s+1)}=\prod_{\{\gamma\}_{p}}\left[1-\chi_{\gamma}^{q} e^{-s l_{\gamma}}\right], \quad(\operatorname{Re}(s)>1) .
$$

To study the analytic properties of $Z_{0}$ and $Z_{1}$ let us consider the Selberg supertrace formula for $m=0$, i.e. ${ }^{2}$

$$
\begin{aligned}
& \sum_{n=0}^{\infty}\left[h\left(p_{n}^{B}\right)-h\left(p_{n}^{F}\right)\right]=i(g-1) \int_{-\infty}^{\infty} h\left(i p+\frac{1}{2}\right) \tanh \pi p d p \\
+ & \sum_{\{\gamma\}_{r}} \sum_{k=1}^{\infty} \frac{l_{\gamma}}{\epsilon^{\frac{k l_{\gamma}}{2}}-\epsilon^{-\frac{k l_{\gamma}}{2}}}\left[g\left(k l_{\gamma}\right)+g\left(-k l_{\gamma}\right)-\chi_{\gamma}{ }^{k}\left(g\left(k l_{\gamma}\right) e^{-\frac{k l_{\gamma}}{2}}+g\left(-k l_{\gamma}\right) \epsilon^{\frac{k i_{\gamma}}{2}}\right)\right] .
\end{aligned}
$$

${ }^{1}$ If not otherwise noted, I do not distinguish between the these two numbers $l_{\gamma}$ in Eq.(1) and Eq.(12). ${ }^{2}$ 'Througout this chapter I denote by $\lambda^{B(F)}=\frac{1}{2}+p_{n}^{B(F)}(n \in N)$ the Bose and Fermi Eigenvalues of $\square$, respectively.
To get informations for $Z_{1}$ or $R_{1}$, respectively, one has to choose a test function $h(p)$ so that the first two terms in the square bracket in the supertrace formula cancel, i.c. $g(u)=-g(-u)$. I choose the function $(\operatorname{Re}(s) \cdots 1, \operatorname{Re}(\sigma) \div 1)$ :

$h_{s}(p)=\left.2\left(\lambda-\frac{1}{2}\right)\left[\frac{1}{s^{2}-\left(\lambda-\frac{1}{2}\right)^{2}}-\frac{1}{\sigma^{2}-\left(\lambda \cdot \frac{1}{2}\right)^{2}}\right]\right|_{\lambda-\frac{1}{2}+t p}=2 i p\left(\frac{1}{s^{2}-p^{2}}-\frac{1}{\sigma^{2}+p^{2}}\right)$.

The secund term plays the role of a regulator in order that all the involved terms in the supertrace formula are convergent. Thus for $g(u)$ :

$$
g(u)=\frac{1}{2 \pi} \int_{-\infty}^{\infty} \epsilon^{-i u p} h_{s}(p) d p=\frac{2}{\pi} \int_{0}^{\infty} p \sin u p\left(\frac{1}{s^{2}+p^{2}}-\frac{1}{\sigma^{2}+p^{2}}\right) d p,
$$

and therefore $g(u)$ is an odd function as required. Using $[33, p .406$;

$$
\int_{0}^{\infty} \frac{x \sin a x}{\beta^{2}+x^{2}} d x=\frac{\pi}{2} \epsilon^{-a, 3}
$$

I get $(u>0) g(u)=\left(\epsilon^{-s u}-\epsilon^{-\sigma u}\right)$ and for $u \in \mathbf{R}$

$$
g(u)=\operatorname{sign}(u)\left(e^{-s|u|}-e^{-o|u|}\right) .
$$

finally for $G(u, \chi)$

$$
G\left(u, \chi_{\gamma}\right)=2 \chi_{\gamma}\left(e^{-s|u|} \ldots e^{-o|u|}\right) \sinh \frac{u}{2} .
$$

Thus only the $x_{\gamma}$-term remains in the supertrace formula which allows to study the properties of $Z_{1}$ alone. Inserting $G(u, x)$ into the length term yields

$$
\begin{aligned}
& \sum_{\{\gamma\}_{p}} \sum_{k=1}^{\infty} \frac{l_{\gamma}}{2 \sinh \frac{k l_{\gamma}}{2}} G\left(k l_{\gamma}, x_{\gamma}\right)=\sum_{\{\gamma\}_{p}} \sum_{k=1}^{\infty} l_{\gamma, x_{\gamma}}{ }^{k}\left(e^{-s k l_{\gamma}}-\epsilon^{-\sigma k l_{\gamma}}\right) \\
= & \sum_{\{\gamma\}_{p}}\left[\frac{l_{\gamma} \chi_{\gamma} e^{-s l_{\gamma}}}{1-\chi_{\gamma} \epsilon^{-s l_{\gamma}}}-\frac{l_{\gamma} \chi_{\gamma} e^{-\sigma l_{\gamma}}}{1-\chi_{\gamma} e^{-\sigma l_{\gamma}}}\right]=\frac{R_{1}^{\prime}(s)}{R_{1}(s)}-\frac{R_{1}^{\prime}(\sigma)}{R_{1}(\sigma)} .
\end{aligned}
$$

In the last step the property of the logarithmic derivative of the Selberg super $R$ functions has been used, i.e. for $\operatorname{Re}(s)>1$ :

$$
\frac{d}{d s} \ln R_{q}(s)=\frac{d}{d s} \ln \prod_{\{\gamma\}_{p}}\left[1-\chi_{\gamma}{ }^{q} e^{-s l_{\gamma}} \vdots=\sum_{\{\gamma\}_{p}} \frac{l_{\gamma \chi_{\gamma} q^{q} e^{-s l_{\gamma}}}}{1-\chi_{\gamma} q^{q^{-s l_{\gamma}}}} .\right.
$$

The $A_{0}$ term gives

$$
\begin{aligned}
A_{0} & =i(g-1) \int_{-\infty}^{\infty} h_{s}(p) \tanh \pi p d p \\
& =\frac{4}{\pi}(1-g) \int_{0}^{\infty} \frac{d u}{\sinh \frac{u}{2}}\left[\int_{0}^{\infty} \frac{p \sin u p}{p^{2}+s^{2}} d p-\int_{0}^{\infty} \frac{p \sin u p}{p^{2}+\sigma^{2}} d p\right] \\
& =4(1-g) \int_{0}^{\infty} e^{-\frac{i+\sigma}{2} u} \frac{\sinh \frac{\frac{\sigma-s}{2}}{\sinh \frac{u}{2}}}{d u},
\end{aligned}
$$


where the integrals (17) and (IV.51) have been used. Using now [33, p.356

$$
\int_{0}^{\infty} e^{-\mu x} \frac{\sinh \beta x}{\sinh b x} d x=\frac{1}{2 b}\left[\Psi\left(\frac{1}{2}+\frac{\mu+\beta}{2 b}\right)-\Psi\left(\frac{1}{2}+\frac{\mu-\beta}{2 b}\right)\right]
$$

where $\Psi(z)=\Gamma^{\prime}(z) / \Gamma(z), z \in \mathbf{C}$, I get finally for $A_{0}$

$$
A_{0}=4(g-1)\left[\Psi\left(s+\frac{1}{2}\right)-\Psi\left(\sigma+\frac{1}{2}\right)\right] .
$$

Let us denote by $\Delta n_{0}^{(0)}=n_{0}^{B}-n_{0}^{F}$ the difference between the number of even and odd zero modes of the Dirac operator $\square$. Thus the supertrace formula for the function $h_{s}$ reads

$$
\begin{aligned}
\sum_{n=1}^{\infty}\left[h_{s}\left(p_{n}^{B}\right)-h_{s}\left(p_{n}^{F}\right)\right]- & \Delta n_{0}^{(0)}\left[\frac{1}{\left(s-\frac{1}{2}\right)\left(s+\frac{1}{2}\right)}-\frac{1}{\left(\sigma-\frac{1}{2}\right)\left(\sigma+\frac{1}{2}\right)}\right] \\
& =\mathbf{4}(g-1)\left[\Psi\left(s+\frac{1}{2}\right)-\Psi\left(\sigma+\frac{1}{2}\right)\right]+\frac{R_{1}^{\prime}(s)}{R_{1}(s)}-\frac{R_{1}^{\prime}(\sigma)}{R_{1}(\sigma)} .
\end{aligned}
$$

First I discuss the trivial structure of zeros and poles of $R_{1}$ and $Z_{1}$. I read off the analytic properties of the $R_{1}$-function:

- : For $s=\frac{1}{2}$ : there is a pole, zero or a regular point depending on whether $\Delta n_{0}^{(0)}>0, \Delta n_{0}^{(0)}<0$ or $\Delta n_{0}=0$, respectively.

- : For $s=-\frac{1}{2}$ : there is a zero of multiplicity $4(g-1)+\Delta n_{0}^{(0)}$ (assuming that $\left.\left|\Delta n_{0}\right|<4(g-1)\right)$

- : For $s=-\frac{1}{2}-k(k \in \mathrm{N})$ : there are zeros with multiplicity $4(g-1)$.

In the discussion has been used that $\left.\operatorname{Res} \Psi(z)\right|_{z=-n}=-1\left(k \in \mathbf{N}_{0}\right)$. Therefore the analytic properties of $Z_{1}$ read:

- : For $s=\frac{1}{2}$; there is a pole, zero or a regular point depending on whether $\Delta n_{0}^{(0)}>0, \Delta n_{0}^{(0)}<0$ or $\Delta n_{0}=0$, respectively.

- : For $s=-\frac{1}{2}-k\left(k \in \mathbf{N}_{0}\right)$ : there are zeros with multiplicity $4(k+1)(g-1)$

Second let us turn to the nontrivial zeros and poles of these two functions (first so called "small Eigenvalues" not considered). Since

$$
h_{s}(p)=2 i p\left[\frac{1}{(s+i p)(s-i p)}-\frac{1}{(\sigma+i p)(\sigma-i p)}\right]
$$

we have

$$
\left.\operatorname{Res}\left[h_{s}\left(p_{n}^{B}\right)\right]\right|_{s=i p_{n}^{B}}=1,\left.\quad \operatorname{Res}\left[h_{s}\left(p_{n}^{B}\right)\right]\right|_{s=-i p_{n}^{B}}=-1
$$

with signs of the residua reversed for the Fermi Eigenvalues. Thus $R_{1}(s)$ has

- : for $s=i p_{n}^{B(F)}$; there are zeros (poles) with the same multiplicity as the corresponding Eigenvalue of $\square$.

- : for $s=\cdots i p_{n}^{B(F)}$ : reversed situation for poles and zeros.
Note the crucial dependence on the signs.

Since $R_{1}(1 \pm i p)$ is regular we can conclude by $Z_{1}(1+i p)=R_{1}(1 \pm i p) \cdot Z_{1}(2 \pm i p)$ that $Z_{1}(s)$ is regular on the line $\operatorname{Re}(s)=1$. Furthermore this gives by $Z_{1}(i p)=$ $R_{1}(i p) \cdot Z_{1}(1+i p)$ that $Z_{1}(s)$ has on the line $\operatorname{Re}(s)=0$ the same properties as $R_{1}(s)$, i.e. zéros (poles) for $s=i p_{n}^{B(F)}$ and poles (zeros) for $s=-i p_{n}^{B(F)}$. Repeating this procedure for $Z_{1}(i p-k)=R_{1}(i p-k) \cdot Z_{1}(i p-k+1)(k \in \mathbf{N})$, we get an infinite number of critical lines for $Z_{1}$ located at $\operatorname{Re}(s)=-k\left(k \in \mathrm{N}_{0}\right)$. Therefore the analytic properties of $Z_{1}$ for the nontrivial zeros and poles $\left(k \in \mathbf{N}_{0}\right)$ read:

- : For $s=i p_{n}^{B(F)}-k$ : there are zeros $\left(p_{N}^{B}\right)$ and poles $\left(p_{n}^{F}\right)$ with the sane nultiplicity as the corresponding Eigenvalue of $\square$.

-: For $s=-i p_{n}^{B(F)}-k$; there are poles $\left(p_{N}^{B}\right)$ and zeros $\left(p_{n}^{F}\right)$ with the same multiplicity as the corresponding Eigenvalue of $\square$

Figure 3: Zeros and poles of the zeta-function $Z$

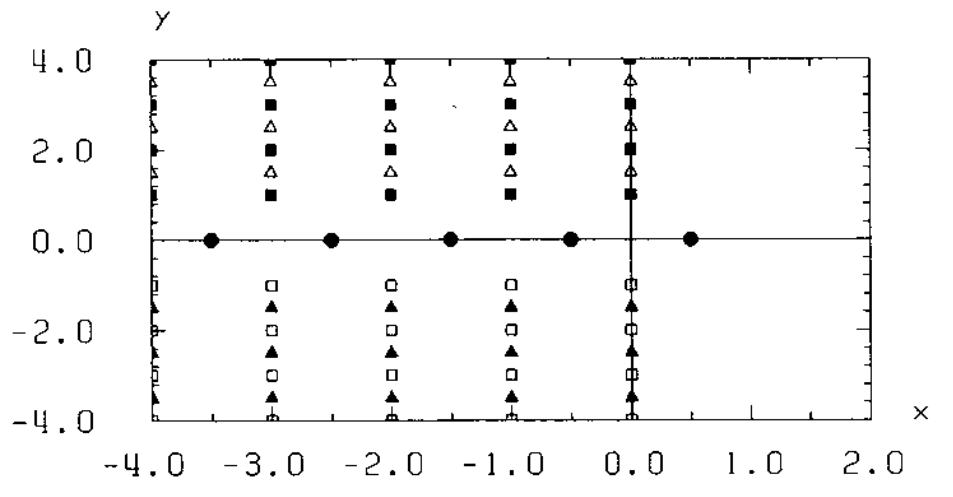

Finally let us discuss the case of so called small Eigenvalues $\left(0 \leq \lambda \leq \frac{1}{2}\right)$, which are also unknown and likely do not exist for small $g[\bar{\tau}]$. We can see from Eq.(25) that for $R_{1}$ they are located at $-\frac{1}{2} \leq s \leq \frac{1}{2}$ :

- : for $s= \pm\left(\lambda_{n}^{B}-\frac{1}{2}\right)$ there are zeros (poles) and

- : for $s=\mp\left(\lambda_{n}^{F}-\frac{1}{2}\right)$ there are poles (zeros) with the same multiplicity as the corresponding Eigenvalue of $\square$, respectively.

By the same considerations as for the other nontrivial zeros and poles we get the structure for the $Z_{1}$-function $\left(k \in \mathbf{N}_{0}\right)$ :

- : for $s=\lambda_{n}^{B(F)}-\frac{1}{2}-k$ there are zeros (poles) and

- : for $s:=-\left(\lambda_{n}^{B(F)}-\frac{1}{2}\right)-k$ there are poles (zeros) with the same multiplicity as the corresponding Eigenvalue of $\square$, respectively.

All these Eigenvalues are of course, even numbers, i.e. clements of $\mathbf{C}_{c}$. Therefore the supertrace formula can be extended meromorphically to all $s \in A_{\infty}$ and $R_{1}$ and $Z_{1}$ are meromorphic functions in $\Lambda_{\infty}$. 
In figure 3 the analytic properties of the $Z_{1}$-function are displayed. The trivial zerus are indicated by filled dots, the position of the bosonic zeros and poles by filled and cmpty squares, respectively, and the position of the fermionic zeros and poles by filled and empty triangles, respectively. The small Eigenvalues are not considered. The $x$ and $y$-axis are taken at the body of $\Lambda_{\infty}$, i.e. $\left(\Lambda_{\infty}\right)_{B \text { ody }}=\mathrm{C}$. The $y$-axis is taken in arbitrary units.

Let us consider $\mathrm{Eq}$. (25) in the limit $\sigma \rightarrow \frac{1}{2}$ and get

$$
\lim _{\sigma \rightarrow \frac{1}{2}}\left[\frac{R_{1}^{\prime}(\sigma)}{R_{1}(\sigma)}+\frac{\Delta n_{0}^{(0)}}{\sigma^{2}-\frac{1}{4}}-4(g-1) \Psi\left(\sigma+\frac{1}{2}\right)\right]=A_{1}-\gamma E,
$$

where $\Psi(1)=-\gamma_{E}=-0.5 \pi \% 21 \ldots$ is the Euler number and $A_{1}$ is given by

$$
\begin{aligned}
A_{1} & =\frac{R_{1}^{\prime}\left(\frac{1}{2}\right)}{R_{1}\left(\frac{1}{2}\right)}, & \left(\Delta n_{0}^{(0)}=0\right), \\
& =\frac{R_{1}^{\left(1-\Delta n_{0}^{(0)}\right)}\left(\frac{1}{2}\right)}{\left(1-\Delta n_{0}^{(0)}\right) R_{1}^{\left(-\Delta n_{0}^{(0)}\right)}\left(\frac{1}{2}\right)}, & \left(\Delta n_{0}^{(0)}<0\right), \\
& =\frac{\oint\left(\sigma-\frac{1}{2}\right)^{\Delta n_{0}^{(0)}-2} R_{1}(\sigma) d \sigma}{\oint\left(\sigma-\frac{1}{2}\right)^{\Delta n_{0}^{(0)}-1} R_{1}(\sigma) d \sigma} & \left(\Delta n_{0}^{(0)}>0\right) .
\end{aligned}
$$

\section{Therefore}

$$
\begin{aligned}
\sum_{n=1}^{\infty}\left[\frac{2 i p_{n}^{B}}{s^{2}+\left(p_{n}^{B}\right)^{2}} \cdots \frac{2 i p_{n}^{B}}{\frac{3}{4}+\left(p_{n}^{B}\right)^{2}}\right. & \left.-\frac{2 i p_{n}^{F}}{s^{2}+\left(p_{n}^{F}\right)^{2}}+\frac{2 i p_{n}^{F}}{\frac{1}{4}+\left(p_{n}^{F}\right)^{2}}\right]+4(g-1) \gamma_{E}+A_{1} \\
& =\frac{\Delta n_{0}^{(0)}}{\left(s-\frac{1}{2}\right)\left(s+\frac{1}{2}\right)}+4(g-1) \Psi\left(s+\frac{1}{2}\right)+\frac{R_{1}^{\prime}(s)}{R_{1}(s)}
\end{aligned}
$$

$h_{s}$ has the symmetry $h_{s}=h_{-s}$. Writing down Eq.(30) for $s \rightarrow-s$ and subtracting it from Eq.(30) gives with $\Psi\left(\frac{1}{2}+s\right)=\Psi\left(\frac{1}{2}-s\right)+\pi \tan \pi s[55, \mathrm{p} .14]$ the functional equation in differential form for the $R_{1}$-function

$$
\frac{d}{d s} \ln R_{1}(s) R_{1}(-s)=-4(g-1) \pi \tan \pi s .
$$

Of course, every information about the nontrivial zeros is lost. This equation can be integrated yielding

$$
R_{1}(s) R_{1}(-s)=\tilde{A}_{1}(\cos \pi s)^{4(g-1)}
$$

where $\tilde{A}_{1}$ is a constant given e.g. by $\tilde{A}_{1}=R_{1}\left(s_{0}\right) R_{1}\left(-s_{0}\right)\left(\cos \pi s_{0}\right)^{4(1-g)}$ with some $s_{0} \in \mathbf{C}$, which is however, independent of $s_{0}$. I have, e.g. (no small Eigenvalue $\lambda=\frac{1}{2}$ assumed) for $s_{0}=0: \bar{A}_{1}=R_{1}^{2}(0)$.

\section{The Selberg Super Zeta-Function $Z_{v}$}

In this section I derive the analytic properties of the Selberg super zeta-function $Z_{0}$ and present a functional equation connecting the two Selberg super zeta-functions $Z_{0}$ and $Z_{3},{ }^{1} I$ considier the test function $\left(\operatorname{Re}(s) \cdot \frac{3}{2}\right)$ :

$$
h_{s}(p)=\frac{1}{\lambda(1-\lambda) \cdots s(1-s)_{\lambda-\imath \mu+\frac{1}{2}}}=\frac{1}{p^{2}+\left(s-\frac{1}{2}\right)^{2}} .
$$

This gives at once $A_{0}=0$ because $h_{s}$ is an even function in $p$. Furthermore for $g(u)$ :

$$
g(u)-\frac{1}{2 \pi} \int_{-\infty}^{\infty} \frac{c^{-i u p}}{\left(s-\frac{1}{2}\right)^{2}+p^{2}} d p=\frac{1}{2 s-1} e^{-\left(s-\frac{1}{2}\right) \mid u !},
$$

$([55, \mathrm{p} .431])$. This gives for $G(u, x)$ :

$$
G\left(k l_{\gamma}, \lambda_{\gamma}\right)=\frac{\epsilon^{-\left(s-\frac{1}{2}\right) k l_{\gamma}}}{s-\frac{1}{2}}\left(1 \cdots \lambda_{\gamma}{ }^{k} \cosh \frac{k l_{\gamma}}{2}\right) .
$$

Therefore the right hand side of the supertrace formula reads

$$
\begin{aligned}
& \sum_{\{\gamma\}_{p}} \sum_{k=1}^{\infty} \frac{l_{\gamma}}{2 \sinh \frac{k l_{\gamma}}{2}} \frac{\epsilon^{-\left(s-\frac{1}{2}\right) k l_{\gamma}}}{s-\frac{1}{2}}\left(1-\chi_{\gamma}{ }^{k} \cosh \frac{k l_{\gamma}}{2}\right) \\
= & \frac{1}{2 s-1} \sum_{\{\gamma\}_{p}} \sum_{k=1}^{\infty} \frac{l_{\gamma}}{1-\epsilon^{-k l_{\gamma}}}\left[2 \epsilon^{-s k l_{\gamma}}-\chi_{\gamma}{ }^{k} e^{-\left(s-\frac{1}{2}\right) k l_{\gamma}} \cdots \chi_{\gamma}{ }^{k} \epsilon^{-\left(s+\frac{1}{2}\right) k l}\right] \\
= & \frac{1}{2 s-1}\left[2 \frac{d \ln Z_{0}(s)}{d s}-\frac{d \ln Z_{1}\left(s-\frac{1}{2}\right)}{d s}-\frac{d \ln Z_{1}\left(s+\frac{1}{2}\right)}{d s}\right] . \\
= & \frac{1}{2 s-1} \frac{d}{d s} \ln \left[\frac{Z_{0}^{2}(s)}{Z_{1}\left(s-\frac{1}{2}\right) Z_{1}\left(s+\frac{1}{2}\right)}\right] .
\end{aligned}
$$

Here the properties of the logarithmic derivative of the super zeta-functions have been used.

$$
\begin{aligned}
& \frac{d}{d s} \ln Z_{q}(s)=\frac{d}{d s} \ln \prod_{\{\gamma\}_{p}} \prod_{k=0}^{\infty}\left[1-x_{\gamma}{ }^{q} \epsilon^{-(s+k) h_{1}}\right] \\
& =\sum_{\{\gamma\}_{p}} \sum_{k=0}^{\infty} \frac{l_{\gamma} \chi_{\gamma}{ }^{q} c^{-(s+k) l_{\gamma}}}{1-\chi_{\gamma}{ }^{q^{-(s+k) l_{\gamma}}}}=\sum_{\{\gamma\}_{p}} \sum_{k=0}^{\infty} \sum_{n=1}^{\infty} l_{\gamma} \chi_{\gamma}{ }^{q n} e^{-(s+k) m l_{\gamma}}
\end{aligned}
$$

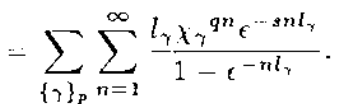

Thus the supertrace formula for the test function $h_{s}$ reads

$$
\sum_{n=1}^{\infty}\left[h_{s}\left(p_{n}^{B}\right)-h_{s}\left(p_{n}^{F}\right)\right] \cdots \frac{\Delta n_{0}^{(0)}}{s(1 \cdots s)}=\frac{1}{2 s-1} \frac{d}{d s} \ln \left[\frac{Z_{0}^{2}(s)}{Z_{1}\left(s-\frac{1}{2}\right) Z_{1}\left(s+\frac{1}{2}\right)}\right] .
$$

It is also possible to derive the analytic properties of $Z_{0}$ similarly as the reasoning for $Z_{1}$ as in section 2. However, this approach here seems to be more straightforward to me. 
Due to our knowledge of the analytic properties of the $Z_{1}$-function we can deduce the analytic properties of the $Z_{0}$-function:

- : $s=-k\left(k \in \mathbf{N}_{0}\right)$ : There are trivial zeros with multiplicity $(g-1)(4 k+2)$.

Since both sides of $\mathrm{Eq} .(38)$ must be regular for $s=\frac{1}{2} \pm i p_{n}-k\left(k \in \mathbf{N}_{0}\right)$ we get further

$\because s=\frac{1}{2}+i p_{n}^{B(F)}-k:$ There are zeros $\left(p_{n}^{B}\right)$ and poles $\left(p_{n}^{F}\right)$,

- $: s=-\frac{1}{2}-i p_{n}^{B(F)}-k:$ there are poles $\left(p_{n}^{B}\right)$ and $z \operatorname{eros}\left(p_{n}^{F}\right)$,

with the same multiplicity as the corresponding Eigenvalue, respectively. Similarly, as for $Z_{1}$, we get an infinite number of critical lines, which makes it very unlikely that a functional equation for $Z_{0}$ exists like Eq.(10) for the ordinary Selberg zeta-function. By the same considerations as for $Z_{1}$ one gets the structure for the $Z_{0}$-function for the "small Eigenvalues" $\left(k \in \mathbf{N}_{0}\right)$ :

- : for $s=\lambda_{n}^{B(F)}-k$ there are zeros (poles) and

- : for $s=1-\lambda_{n}^{B(F)}-k$ there are poles (zeros) with the same multiplicity as the corresponding Eigenvalue of $\square$, respectively.

Figure 4: Zeros and poles of the zeta-function $Z_{0}$

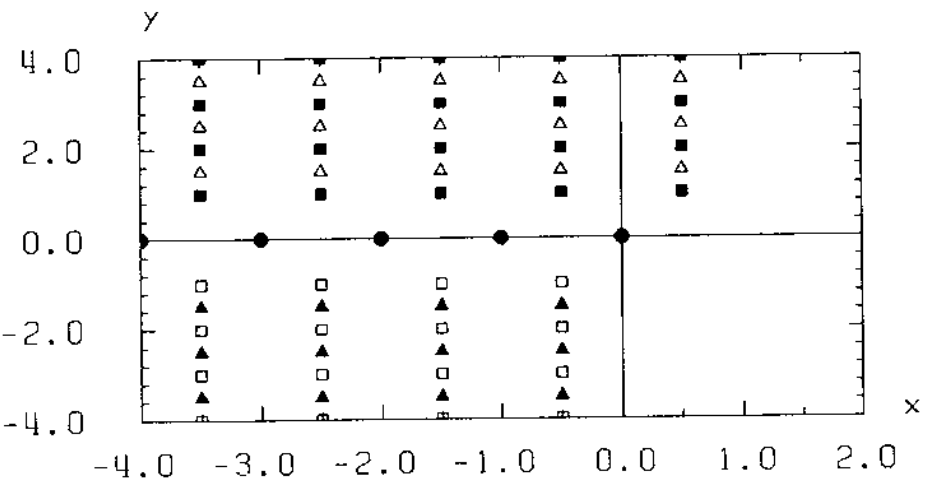

A functional equation for $R_{0}$ can be derived, as can be seen in the next section. Of course Eq. (38) and $Z_{0}$ can be extended meromorphically to all $s \in \Lambda_{\infty}$. In figure 4 the course Eq.(38) and $Z_{0}$ can be extended meromorphicaly to all $s \in \Lambda_{\infty}$. An figure 4 the position of the bosonic zeros and poles by filled and empty squares, respectively, and the position of the fermionic zeros and poles by filled and empty triangles, respectively. The sull Eigenvalues are not considered. The $x$-and $y$-axis are again taken at the body of $\Lambda_{\infty}$, i.e. $\left(\Lambda_{\infty}\right)_{\text {Body }}=\mathrm{C}$. The $y$-axis is taken in arbitrary units.

The test function $h_{s}$ is invariant under the change $s \rightarrow 1-s$. Performing this substitution in $E_{(1 .}(38)$ and subtracting it from (38) yields the functional equation

$$
\frac{d}{d s} \ln \left[\frac{Z_{0}^{2}(s)}{Z_{1}\left(s-\frac{1}{2}\right) Z_{1}\left(s+\frac{1}{2}\right)}\right]=\frac{d}{d s} \ln \left[\frac{Z_{0}^{2}(1-s)}{Z_{1}\left(\frac{1}{2}-s\right) Z_{1}\left(\frac{3}{2}-s\right)}\right]
$$

We consider the functional equation (31) for the $R_{1}$-function and perform the substitution $s \rightarrow \frac{1}{2}-s$. By expressing the $R_{1}$-function by the quotient of the $Z_{1}$-functions, this yields

$$
\frac{d}{d s} \ln \left[\frac{Z_{1}\left(\frac{1}{2}-s\right) Z_{1}\left(s-\frac{1}{2}\right)}{Z_{1}\left(\frac{3}{2}-s\right) Z_{1}\left(s+\frac{1}{2}\right)}\right]=4 \pi(g-1) \cot \pi s .
$$

Thus we find by combining Eqs.(39) and (40) the functional equation in differential form connecting $Z_{0}$ and $Z_{1}$ :

$$
\frac{d}{d s} \ln \left[\frac{Z_{1}\left(\frac{1}{2}-s\right) Z_{0}(s)}{Z_{1}\left(\frac{1}{2}+s\right) Z_{0}(1-s)}\right]=2 \pi(g-1) \cot \pi s . .
$$

The functional equation can be integrated yielding ${ }^{1}$

$$
\frac{Z_{1}\left(\frac{1}{2}-s\right) Z_{0}(s)}{Z_{1}\left(\frac{1}{2}+s\right) Z_{0}(1-s)}=C_{0}(\sin \pi s)^{2(g-1)}
$$

where $C_{0}$ is, e.g. given by $Z_{1}\left(\frac{1}{2}-s_{0}\right) Z_{0}\left(s_{0}\right) /\left[Z_{1}\left(\frac{1}{2}+s_{0}\right) Z_{0}\left(1-s_{0}\right)\left(\sin \pi s_{0}\right)^{2(1-g)}\right]$ with some $s_{0} \in \mathbf{C}$ which is, however independent of $s_{0}$. This gives, e.g. for $s_{0}=\frac{1}{2}: C_{0}=$ $Z_{1}(0) / Z_{1}(1)=R_{1}(0)=\sqrt{\tilde{A}_{1}}$.

\section{The Super Zeta-Function $Z_{S}$}

To get around the difficulties of the combination of the $Z_{0}$ and $Z_{1}$ functions for general test functions $h$ in the Selberg supertrace formula let us (following Matsumoto, Uehara and Yasui [61]) define the super zeta-function $Z_{S}$ :

$$
\begin{aligned}
Z_{S}(s):= & \prod_{\{\gamma\}_{p}} \prod_{n=0}^{\infty} \operatorname{sdet}\left[\mathbf{1}-\operatorname{diag}\left(1, e^{-l_{\gamma}}, \chi_{\gamma} e^{-\frac{l_{\gamma}}{2}}, \chi_{\gamma} e^{-\frac{i_{\gamma}}{2}}\right) e^{-(s+n) l_{\gamma}}\right] \\
& =\prod_{\{\gamma\}_{p}} \prod_{n=0}^{\infty} \frac{\left[1-e^{-(s+n) l_{\gamma}}\right]\left[1-e^{-(s+n+1) l_{\gamma}}\right]}{\left[1-\chi_{\gamma} e^{-\left(s+n+\frac{1}{2}\right) l_{\gamma}}\right]^{2}}=\frac{Z_{0}(s) Z_{0}(s+1)}{Z_{1}^{2}\left(s+\frac{1}{2}\right)}
\end{aligned}
$$

Let us consider the resolvent of $\square_{0}^{2}: R_{s}\left(\square_{0}^{2}\right)=\left(s^{2}-\square_{0}^{2}\right)^{\cdots-1}(\operatorname{Re}(s)>1)$. Therefore

$$
h(p)=\left.\frac{1}{s^{2}-\lambda^{2}}\right|_{\lambda=\frac{1}{2}+i p}=\frac{1}{\left(s^{2}-\frac{1}{4}\right)-i p+p^{2}} .
$$

The Fourier transform of $h(p)$ yields:

$$
g(u)=\frac{1}{2 \pi} \int_{-\infty}^{\infty} h(p) \epsilon^{-i u p} d p=g_{1}(u)+g_{2}(u)
$$

where

$$
\begin{aligned}
& g_{1}(u)=\frac{1}{2 \pi} \int_{-\infty}^{\infty} \frac{\cos u p}{\left(s^{2}-\frac{1}{4}\right)-i p+p^{2}} d p=g_{1}(-u) \\
& g_{2}(u)=\frac{-i}{2 \pi} \int_{-\infty}^{\infty} \frac{\sin u p}{\left(s^{2}-\frac{1}{4}\right)-i p+p^{2}} d p=\cdots g_{2}(-u) .
\end{aligned}
$$


Using the integrals $[33, p .407]$ :

$$
\begin{aligned}
& \int_{-\infty}^{\infty} \frac{(b+c x) \sin a x}{p+q x+x^{2}} d x=\left[\frac{c q-b}{\sqrt{p-q^{2}}} \sin a q+c \cos a q\right] \pi \epsilon^{-a \sqrt{p-q^{2}}} \\
& \int_{-\infty}^{\infty} \frac{(b+c x) \cos a x}{p+q x+x^{2}} d x=\left[\frac{b-c q}{\sqrt{p-q^{2}}} \cos a q+c \sin a q\right] \pi e^{-a \sqrt{p-q^{2}}}
\end{aligned}
$$

we get for $u>0$

$$
\begin{aligned}
& g_{1}(u)=\frac{1}{2 s} \cosh \frac{u}{2} e^{-u s} \\
& g_{2}(u)=\frac{1}{2 s} \sinh \frac{u}{2} e^{-u s} .
\end{aligned}
$$

Therefore $(u \in \mathbf{R})$ :

$$
g(u)=\frac{1}{2 s} e^{\frac{u}{2}-s|u|},
$$

which gives for $G(u, \chi)$

$$
G\left(u, \chi_{\gamma}\right)=\frac{1}{s} \epsilon^{-u s}\left(\cosh \frac{u}{2}-\chi_{\gamma}\right),
$$

and the right hand side of the supertrace formula reads

$$
\frac{1}{2 s} \sum_{\{\gamma\}_{p}} \sum_{k=1}^{\infty} \frac{l_{\gamma}}{e^{\frac{k l_{\gamma}}{2}}-e^{-\frac{k l_{\gamma}}{2}}} e^{-s k l_{\gamma}}\left(e^{\frac{k i_{\gamma}}{2}}+e^{-\frac{k t_{\gamma}}{2}}-2 \chi_{\gamma}{ }^{k}\right)=\frac{1}{2 s} \frac{Z_{S}(s)}{Z_{S}(s)} .
$$

For the $A_{0}$ we get

$$
\begin{aligned}
& A_{0}=i(g-1) \int_{-\infty}^{\infty} h(p) \tanh \pi p d p \\
= & i \frac{g-1}{\pi} \int_{0}^{\infty} \frac{d u}{\sinh \frac{u}{2}} \int_{-\infty}^{\infty} \frac{\sin u p}{\left(s^{2}-\frac{1}{4}\right)-i p+p^{2}} d p \\
= & \frac{1-g}{s} \int_{0}^{\infty} \frac{d u}{\sinh \frac{u}{2}} \sinh \frac{u}{2} e^{-u s}=\frac{1-g}{s^{2}},
\end{aligned}
$$

where the integral (47a) has been used. Therefore we have for the resolvent-kernelfunction the supertrace formula

$$
\sum_{n=1}^{\infty}\left[\frac{1}{s^{2}-\left(\lambda_{n}^{B}\right)^{2}}-\frac{1}{s^{2}-\left(\lambda_{n}^{F}\right)^{2}}\right]+\frac{\Delta n_{0}^{(0)}+g-1}{s^{2}}=\frac{1}{2 s} \frac{Z_{S}^{\prime}(s)}{Z_{S}(s)} .
$$

Therefore Eqs. (53) and $Z_{S}$ can be extended meromorplically to all $s \in \Lambda_{\infty}$. The very simple analytic structure of $Z_{S}$ can be read off:

- $: s=0$ there is a zero with multiplicity $2\left(g-1+\Delta n_{0}^{(0)}\right)$,

- $: s= \pm\left(\frac{1}{2}+i p_{n}^{B}\right)$ there are zeros (poles) and

- : $s= \pm\left(\frac{1}{2}+i p_{n}^{F}\right)$ there are poles (zeros), with the same multiplicity as the corresponding Eigenvalue of $\square$. respertively. A very sinple functional relation can be deduced from $\mathrm{Eq} .(53)$. reading

$$
\frac{d \ln Z_{S}(s)}{d s} \quad \frac{d \ln Z_{S}(s)}{d s} .
$$

In toms of $Z_{0}$ and $Z_{1}$ Eq. (54) gives:

$$
\frac{d}{d s} \ln \frac{Z_{0}(s) Z_{0}(s+1)}{Z_{1}^{2}\left(s-\frac{1}{2}\right)}=\frac{d}{d s} \ln \frac{Z_{0}(-s) Z_{0}(1-s)}{Z_{1}^{2}\left(\frac{1}{2}-s\right)} .
$$

Equation (54) or (55), respectively, integrated gives $Z_{S}(s)=Z_{S}(-s)$, thins $Z_{S}(s)$ is an even function in $s$. Combining Eq $\mathrm{s}$. (31), (41) and (55) the functional equation for the $R_{0}$ function is deduced. which reads:

$$
\frac{d}{d s} \ln R_{0}(s) R_{0}(-s):=4 \pi(g-1) \cot \pi s .
$$

Equation (56) can be integrated to give

$$
R_{0}(s) R_{0}(\cdots s)=B_{0}(\sin \pi s)^{4(g-1)} .
$$

where the constant $B_{0}$ is e.g. given by $B_{0}=R_{0}\left(s_{0}\right) R_{0}\left(-s_{0}\right)\left(\sin \pi s_{0}\right)^{4(1-g)}$ with some $s_{0} \in \mathbf{C}$, where $B_{0}$ is independent of $s_{0}$, e.g. for $s= \pm \frac{1}{2}: B_{0}=Z_{0}\left(-\frac{1}{2}\right) / Z_{0}\left(\frac{3}{2}\right)=$ $R_{0}\left(-\frac{1}{2}\right)$. Of course, any information about the nontrivial zeros and poles is lost. Note that the same relation holds also for the ordinary Selbcrg zeta-function; however, in this case the integration constant is given by $B=2^{4(g-1)}$, sec Eq.(11).

From Eqs.(32), (42) and (57) many relations linking $Z_{0}$ and $Z_{1}$ for particular arguments can be deduced, e.g.

$$
B_{0}=\frac{Z_{0}\left(-\frac{1}{2}\right)}{Z_{0}\left(\frac{3}{2}\right)}=\frac{Z_{1}(-1) Z_{1}(1)}{Z_{1}(2) Z_{1}(0)}=\frac{Z_{1}^{2}(0)}{Z_{1}^{2}(1)}=C_{0}^{2}=\tilde{A}_{1} .
$$

However, I do not see any valuable consequence as, e.g. determining from these relations the constants $\dot{A}_{1}, B_{0}$ and $C_{0}$ like for the Selberg zeta-function. It is also not obvious to me to derive from these relations a functional relation for $Z_{0}$ or $Z_{1}$ respectively, like $\mathrm{Eq}_{\mathrm{q}}$ (10) for the ordinary Selberg zeta-function. On the contrary: I believe that such relations do not exist for $Z_{0}$ and $Z_{1}$, because we have an infinite number of critical lines for these two funtions. The immediate consequence of such relations, if they would exist, would be that we could solve the Eigenvalue problem for the operator $\square_{0}$ by just looking at the poles ( for $\lambda_{n}^{B}$ ) and zeros (for $\lambda_{n}^{F}$ ) at, e.g. the critical line $\operatorname{Re}(s)=-\frac{1}{2}$ for $Z_{0}(s)$. The values at the critical line $\operatorname{Re}(s)=-\frac{1}{2}$ for $Z_{0}(s)$ would be related to the line $\operatorname{Re}(s)=\frac{3}{2}$, where $Z_{0}$ could be easily calculated by Eq. (12) once a sufficient large enough set of geodesics $\left\{l_{\gamma}\right\}$ would be known. This is, however, very unlikely (but not a proof).

${ }^{1}$ In comparision to Ref.[61] one has to take the limit $a \because 1$ in llie formulas. 


\section{SPECTRa and Determinants OF LAPLACE-DIrac Operators}

\section{Resolvent and Heat-Kerne}

Since $\square_{m}^{2}$ is not a positive definite operator I calculate the superdeterminant of $c^{2}-\square_{m}^{2}$ for $\operatorname{Re}(c)>m$ and analytically continue in $c$. Similar considerations have been done by Aoki [3] by means of the supertrace of the heat kernel of $\square_{m}^{2}$. Fitted with the knowledge of the analytical properties of the Selberg super zeta-functions I can avoid the indirect reasoning of Aoki to get the superdeterminants in compact form. For this purpose the functional relations for $Z_{0}$ and $Z_{1}$ of the previous chapter are used. These functional relations have not been available in [3]; without proof Aoki has used the functional relation of the Selberg zeta-function, assuming that it is also valid in the super case. As discussed at the end of the previous section this seems to be very unlikely that such a functional relation exist. Furthermore, statements of the spectrum of the operators $\hat{\square}_{m}$ and its relation to the spectrum of $\square$ can be made (and similarly for $\square_{m}$ which I do not consider explicitly).

Let be $m \in \mathbf{N}_{0}$. The superdeterminant is defined using the $\zeta$-function regularization as ${ }^{1}$

$$
\begin{aligned}
\operatorname{sdet}\left(c^{2}-\square_{m}^{2}\right) & =\exp \left[-\left.\frac{\partial}{\partial s} \zeta_{m}(s ; c)\right|_{s=0}\right] \\
\zeta_{m}(s ; c) & =\operatorname{str}\left[\left(c^{2}-\square_{m}^{2}\right)^{-s}\right] \\
& =\frac{1}{\Gamma(s)} \int_{0}^{\infty} d t t^{s-1} \operatorname{str}\left\{\exp \left[-t\left(c^{2}-\square_{m}^{2}\right)\right]\right\}
\end{aligned}
$$

where use has been made of the integral $[33, \mathrm{p} .317]$ :

$$
\int_{0}^{\infty} x^{\nu-1} e^{-\mu x} d x=\mu^{-\nu} \Gamma(\nu)
$$

So one has two possibilities to study sdet $\left(c^{2}-\square_{m}^{2}\right)$ :

1) by computing $\operatorname{str}\left[\left(c^{2}-\square_{m,}^{2}\right)^{-s} \mid\right.$ (generalized resolvent) or

2) by computing $\operatorname{str}\left\{\exp \left[-t\left(c^{2}-\square_{m}^{2}\right)\right]\right\}$ (heat-kernel).

I prefer the second method. It has the advantage that integration constants are explicitly given (see below). The function $h$ corresponding to the heat-kernel of $c^{2}-$ $\square_{m}^{2}$ read

$$
h_{h k}(s)=\epsilon^{t\}\left(s+\frac{m}{2}\right)^{2}-c^{2} !}
$$

Therefore for $g(u)$

$$
\begin{aligned}
g(u) & =\frac{1}{2 \pi} \int_{-\infty}^{\infty} \epsilon^{-i u p} h_{h k}\left(i p+\frac{1}{2}\right) d p \\
& =\frac{1}{2 \pi} \epsilon^{\left.t ! \frac{\pi+1}{2}\right)^{2}-c^{2} !} \int_{-\infty}^{\infty} \epsilon^{\left.-p^{2} t+i(m+1) t-u\right] p} d p \\
& =\frac{1}{\sqrt{4} \pi t} \exp \left[-\frac{u^{2}}{4 t}-c^{2} t+(m+1) \frac{u}{2}\right]
\end{aligned}
$$

This method of regularization of determinants by zeta-functions was introduced by Ray and Singer 73 in differential geometry and Hawking 44 in in field theory.
This gives

$$
\begin{gathered}
G(u, \chi)=\frac{1}{\sqrt{\pi t}} e^{-u^{2} / 4 t-c^{2} t}\left[\cosh (m+1) \frac{u}{2}-\chi \cosh m \frac{u}{2}\right] \\
g(u)-g(-u)=\frac{1}{\sqrt{\pi t}} e^{-u^{2} / 4 t-c^{2} t} \sinh (m+1) \frac{u}{2} .
\end{gathered}
$$

Splitting now the calculation of $\zeta_{m}(s ; c)$ into two terms corresponding to the identity transformation and the length term, respectively, gives:

$$
\zeta_{m}(s ; c)=\zeta_{m}^{I}(s ; c)+\zeta_{m}^{\Gamma}(s ; c) .
$$

I first calculate $\zeta_{m}^{I}$ :

$$
\begin{aligned}
\zeta_{m}^{I}(s ; c) & =\frac{1}{\Gamma(s)} \int_{0}^{\infty} t^{s-1} A_{0}^{(m)}(t) d t \\
A_{0}^{(m)}(t) & =\frac{1-g}{\sqrt{\pi t}} e^{-c^{2} t} \int_{0}^{\infty} e^{-\frac{u^{2}}{4 t} \frac{\sinh (m+1) \frac{u}{2}}{\sinh \frac{u}{2}} \cosh m \frac{u}{2} d u} \\
& =(1-g) e^{-c^{2} t} \sum_{k=0}^{m} e^{k^{2} t} .
\end{aligned}
$$

Equation ( 7 ) can be proved by induction:

First step: $m=0$ :

$$
A_{0}^{(0)}(t)=\frac{1-g}{\sqrt{\pi t}} \int_{0}^{\infty} e^{-u^{2} / 4 t} d u=(1-g) e^{-c^{2} t} .
$$

Second step: $m \rightarrow m+1$ :

$$
\begin{aligned}
& A_{0}^{(m+1)}(t)=\frac{1-g}{\sqrt{\pi t}} \epsilon^{-c^{2} t} \int_{0}^{\infty} \frac{e^{-u^{2} / 4 t}}{\sinh \frac{u}{2}} \sinh (m+2) \frac{u}{2} \cosh (m+1) \frac{u}{2} d u \\
& =\frac{1-g}{\sqrt{\pi t}} \epsilon^{-c^{2} t} \int_{0}^{\infty} \frac{\epsilon^{-u^{2} / 4 t}}{\sinh \frac{u}{2}} \cdot \cosh (m+1) u \sinh \frac{u}{2}+\sinh (m+1) \frac{u}{2} \cosh m \frac{u}{2} j d u \\
& =A_{0}^{(m)}+\frac{1-g}{\sqrt{\pi t}} \epsilon^{-c^{2} t} \int_{0}^{\infty} \epsilon^{-u^{2} / 4 t} \cosh (m+1) u d u \\
& =(1-g)\left[\epsilon^{-c^{2} t} \sum_{k=0}^{m} \epsilon^{k^{2} t}+\epsilon^{-c^{2} t+(m+1)^{2} t}\right]=(1-g) \epsilon^{-c^{2} t} \sum_{k=0}^{m+1} \epsilon^{k^{2} t}
\end{aligned}
$$

where the integral $\{33$, p. $317:$

$$
\int_{0}^{\infty} \epsilon^{-\beta x^{2}} \cosh \alpha x d x=\frac{1}{2} \sqrt{\frac{\pi}{\beta}} \epsilon^{\alpha^{2} / 4 \beta}
$$

has been used

Similarly:

$$
\begin{aligned}
A_{0}^{(-1)} & =0 \\
A_{0}^{(-m)}(t) & =(g-1) \epsilon^{-c^{2} t} \sum_{k=0}^{m-2} \epsilon^{k^{2} t} \quad(m=2.3 \ldots) .
\end{aligned}
$$


This gives for $\zeta_{m}^{I}$ :

$$
\zeta_{m}^{I}(s ; c)=\frac{1-g}{\Gamma(s)} \sum_{k=0}^{m} \int_{0}^{\infty} t^{s-1} \epsilon^{-\left(c^{2}-k^{2}\right) t} d t=(1-g) \sum_{k=0}^{m}\left(c^{2}-k^{2}\right)^{-s}
$$

For later use it is easily calculated

$$
\left.\frac{\partial}{\partial s} \zeta_{m}^{I}(s ; c)\right|_{s=0}=(g-1) \sum_{k=0}^{m} \ln \left(c^{2}-k^{2}\right)
$$

Let us calculate $\zeta_{m}^{\mathrm{r}}$ in two alternative ways. The first is appropriate to the analysis of the spectrum, the second to the calculation of the superdeterminants.

1) The supertrace formula for the heat-kernel now reads:

$$
\begin{aligned}
& \sum_{n=1}^{\infty}\left\{\epsilon^{t\left[\left(\lambda_{n, m}^{B}\right)^{2}-c^{2}\right]}-e^{\left[t\left(\lambda_{n, m}^{F}\right)^{2}-c^{2}\right]}\right\}=(1-g) e^{-c^{2} t} \sum_{k=0}^{m} e^{k^{2} t} \\
& +\frac{\epsilon^{-\epsilon^{2} t}}{\sqrt{4 \pi t}} \sum_{\gamma \in \Gamma} \sum_{k=1}^{\infty} \frac{l_{\gamma \lambda_{\gamma}}{ }^{k m}}{\sinh \frac{k l_{\gamma}}{2}} e^{-\frac{k^{2} l_{\gamma}{ }^{2}}{4 t}}\left[\cosh (m+1) j \frac{k l_{\gamma}}{2} \cdots x_{\gamma}{ }^{k} \cosh m \frac{k l_{\gamma}}{2}\right]
\end{aligned}
$$

and the $A_{0}$ term appropriately replaced for negatjve integers. With the help of Eqs.(2), (7) and the integral $[33$, p.340]:

$$
\int_{0}^{\infty} x^{\nu-1} e^{-\frac{\beta}{x}-\gamma x} d x=2\left(\frac{\beta}{\gamma}\right)^{\frac{\nu}{2}} K_{\nu}(2 \sqrt{\beta \gamma})
$$

this gives for the supertrace formula of the generalized resolvent kernel:

$$
\begin{aligned}
& \sum_{n=0}^{\infty}\left[\frac{1}{\left[c^{2}-\left(\lambda_{n, m}^{B}\right)^{2 i s}\right.}-\frac{1}{\left[c^{2} \cdots\left(\lambda_{n, m}^{F}\right)^{2}\right]^{s}}\right] \\
& =(1-g) \sum_{k=0}^{m} \frac{1}{\left(c^{2}-k^{2}\right)^{s}}+\frac{2}{\Gamma(s)} \sum_{\gamma \in \Gamma} \sum_{k=1}^{\infty} \frac{l_{\gamma} \lambda_{\gamma}{ }^{k m}}{e^{\frac{k l_{\gamma}}{2}}-e^{-\frac{k l_{\gamma}}{2}}}\left(\frac{k l_{\gamma}}{2 c}\right)^{s-\frac{1}{2}} \\
& \times K_{-\frac{1}{2}}\left(c k l_{\gamma}\right)\left[\cosh \left(\frac{m+1}{2} k l_{\gamma}\right)-\lambda_{\gamma}{ }^{k} \cosh \left(\frac{m}{2} k l_{\gamma}\right)\right] .
\end{aligned}
$$

This gives explicitly for $s=1(\mathrm{~m}$ even $)$ :

$$
\begin{aligned}
& \sum_{n=0}^{\infty}\left[\frac{1}{c^{2}-\left(\lambda_{n, m}^{B}\right)^{2}}-\frac{1}{c^{2}-\left(\lambda_{n, m}^{F}\right)^{2}}\right]+(g-1) \sum_{k=0}^{m} \frac{1}{c^{2}-k^{2}} \\
& \quad=\frac{1}{2 c \sqrt{\pi}} \sum_{\gamma \in \Gamma} \sum_{k=1}^{\infty} \frac{l_{\gamma}}{e^{\frac{k l_{\gamma}}{2}}-e^{-\frac{k l_{\gamma}}{2}}} e^{-k c l_{\gamma}}\left[\cosh \left(\frac{m+1}{2}-k l_{\gamma}\right)-\lambda_{\gamma}{ }^{k} \cosh \left(\frac{m}{2} k l_{\gamma}\right)\right] \\
& \quad=\frac{1}{2 c} \frac{d}{d c} \ln \left[\frac{Z_{0}\left(\frac{m}{2}+c+1\right) Z_{0}\left(c-\frac{m}{2}\right)}{Z_{1}\left(c+\frac{m+1}{2}\right) Z_{1}\left(c+\frac{1-m}{2}\right)}\right]
\end{aligned}
$$

where Eg. (V.3i) has been used (logaritmic derivative of the super zcta-functions). For $s=1$ and $m$ odd

$$
\begin{aligned}
\sum_{n=0}^{\infty}\left[\frac{1}{c^{2}-\left(\lambda_{n}^{B}\right)^{2 !}}-\frac{1}{c^{2}-\left(\lambda_{n}^{F}\right)^{2 !}}\right]+ & (g-1) \sum_{k=0}^{m} \frac{1}{c^{2}-k^{2}} \\
& =\frac{1}{2 c} \frac{d}{d c} \ln \left[\frac{Z_{1}\left(\frac{m}{2}+c+1\right) Z_{1}\left(c-\frac{m}{2}\right)}{Z_{0}\left(\frac{m+1}{2}+c\right) Z_{0}\left(c+\frac{1-\frac{m}{2}}{2}\right)}\right]
\end{aligned}
$$

2) Let first $m$ be an even number. Let us consider the representation $(\operatorname{Re}(s)<1)$ :

$$
t^{s-1}=\frac{2}{\Gamma(1-s)} \int_{0}^{\infty} \frac{\lambda+c}{[\lambda(\lambda+2 c) s} e^{-\lambda(\lambda+2 c) t} d \lambda
$$

This integral representation follows with the help of $[33, \mathrm{p} .318(\operatorname{Re}(\nu)>-1)$ :

$$
\int_{u}^{\infty}(x-u)^{\nu} c^{-\mu x} d x=\mu^{-\nu-1} \Gamma(\nu+1) \epsilon^{-u \mu} .
$$

Therefore we get for $\zeta_{m}^{\Gamma}(c ; s)$ with the help of Eq.(14) and the represent ation $K_{ \pm \frac{1}{2}}(\tilde{z})=$ $\sqrt{\pi / 2 z c^{-2}:}$

$$
\begin{aligned}
& \zeta_{m}^{\Gamma}(s ; c)=\frac{\sin \pi s}{\pi} \int_{0}^{\infty} \frac{d \lambda}{[\lambda(\lambda+2 c)]^{s}} \\
& \times 2 \sum_{\gamma \in \Gamma} \sum_{k=1}^{\infty} 1 \frac{l_{\gamma}}{\cdots-c^{-k} l_{\gamma}} c^{-k l_{\gamma}\left(\lambda+c+\frac{1}{2}\right)}\left[\cosh \left(\frac{m+1}{2} k l_{\gamma}\right)-x_{\gamma}{ }^{k} \cosh \left(\frac{m}{2} k l_{\gamma}\right)\right] \\
& =\frac{\sin \pi s}{\pi} \int_{0}^{\infty} \frac{d \lambda}{\lambda(\lambda+2 c)]^{s}} \frac{d}{d \lambda} \ln \left[\frac{Z_{0}\left(\frac{m}{2}+\lambda+c+1\right) Z_{0}\left(\lambda+c-\frac{m}{2}\right)}{Z_{1}\left(\frac{m+1}{2}+\lambda+c\right) Z_{1}\left(\frac{m-1}{2}+\lambda+c\right)}\right] .
\end{aligned}
$$

where $\mathrm{E}_{\mathrm{q}}(\mathrm{V} .3 \overline{\mathrm{T}})$ has been used again. Let be $\left.f(s)=\sin (\pi s)[\lambda(\lambda+2 c)]^{-s}\right]$. Then $f^{\prime}(s): s=\pi$ and we get for $\zeta^{\prime}(0 ; c)(\operatorname{Re}(s)>m)$ :

$$
\begin{aligned}
& \zeta^{\prime}(0 ; c)=(g-1) \sum_{k=0}^{m} \ln \left(c^{2}-k^{2}\right) \\
& \quad+\int_{0}^{\infty} d \lambda \frac{d}{d \lambda} \ln \left[\frac{Z_{0}\left(\lambda+\frac{m}{2}+c+1\right) Z_{0}\left(\lambda-\frac{m}{2}+c\right)}{Z_{1}\left(\lambda+\frac{m+1}{2}+c\right) Z_{1}\left(\lambda+\frac{1-\frac{m}{m}}{2}+c\right)}\right] \\
& =(g-1) \sum_{k=0}^{m} \ln \left(c^{2}-k^{2}\right) \cdots \ln \left[\frac{Z_{0}\left(c+1+\frac{m}{2}\right) Z_{0}\left(c-\frac{m}{2}\right)}{Z_{1}\left(c+\frac{m+3}{2}\right) Z_{1}\left(c+\frac{1-\frac{m}{m}}{2}\right)}\right] .
\end{aligned}
$$

Here it has been have used that $\lim _{s \rightarrow \infty} Z_{q}(s)=1$, which follows at once from the Euler product representation of the Selberg super zeta-functions. Therefore $(m=$ $0,2, \ldots)$ :

$$
\operatorname{sdet}\left(c^{2}-\square_{m}^{2}\right)=\frac{Z_{0}\left(c+\frac{m_{2}}{2}+1\right) Z_{0}\left(c-\frac{m}{2}\right)}{Z_{1}\left(c-\frac{m+1}{2}\right) Z_{1}\left(c-\frac{1-m}{2}\right) c^{2(g-1)}} \prod_{k=1}^{m}\left(c^{2}-k^{2}\right)^{2-g} .
$$


Similarly $(m=2,4, \ldots)$ :

$$
\operatorname{sdet}\left(c^{2}-\square_{-m}^{2}\right)=\frac{Z_{0}\left(c-\frac{m}{2}+1\right) Z_{0}\left(c+\frac{m}{2}\right)}{Z_{1}\left(c+\frac{m+1}{2}\right) Z_{1}\left(c+\frac{1-m}{2}\right)} \prod_{k=0}^{m-2}\left(c^{2}-k^{2}\right)^{g-1}
$$

For $m$ an odd number the roles of $Z_{0}$ and $Z_{1}$ are just reversed and it follows immediately $(m=1,3, \ldots)$

$$
\operatorname{sdet}\left(c^{2}-\square_{m}^{2}\right)=\frac{Z_{1}\left(c+1+\frac{m}{2}\right) Z_{1}\left(c-\frac{m}{2}\right)}{Z_{0}\left(c+\frac{m+1}{2}\right) Z_{0}\left(c+\frac{1-m}{2}\right) c^{2 g-2}} \prod_{k=0}^{m-2}\left(c^{2}-k^{2}\right)^{1-g} .
$$

Similarly $(m=1,3, \ldots)$

$$
\operatorname{sdet}\left(c^{2}-\square_{-m}^{2}\right)=\frac{Z_{1}\left(c+1-\frac{m}{2}\right) Z_{1}\left(c+\frac{m}{2}\right)}{Z_{0}\left(c+\frac{m+1}{2}\right) Z_{0}\left(c+\frac{1-m}{2}\right)} \prod_{k=1}^{m-2}\left(c^{2}-k^{2}\right)^{g-1} .
$$

Equations (23-26) are the starting points for the calculation of determinants. Because the super zeta-functions are meromorphic functions in $\Lambda_{\infty}$, the same holds for the superdeterminants.

Let us denote by $\hat{\Theta}(t):=\operatorname{str}^{\prime}\left[\exp \left(t \square_{0}^{2}\right)\right]$. Thus:

$$
\operatorname{sdet}^{\prime}\left(c^{2}-\square_{0}^{2}\right)=\exp \left\{-\frac{\partial}{\partial s}\left[\left.\frac{1}{\Gamma(s)} \int_{0}^{\infty} d t t^{s-1} e^{-t c^{2} \hat{\Theta}(t)}\right|_{s=0}\right]\right\} .
$$

Some statements about $\hat{\Theta}$ can be made and an equation expressing $\hat{\Theta}$ by the zetafunction $Z_{S}$ can be derived. Let us consider the supertrace formula for the resolvent kernel:

$$
\operatorname{str}^{\prime}\left(c^{2}-\square_{0}^{2}\right)^{-1}=\int_{0}^{\infty} \epsilon^{-t c^{2}} \hat{\Theta}(t) d t=\frac{1}{2 c} \frac{Z_{S}^{\prime}(c)}{Z_{S}(c)}-\frac{g-1+\Delta n_{0}^{(0)}}{c^{2}} .
$$

This equation can be inverted by the theory of Laplace transformations yielding (see e.g. [25, pp.129):

$$
\begin{aligned}
\hat{\Theta}(t)=\frac{1}{2 \pi} \int_{b-i \infty}^{b+\infty} & {\left[\frac{1}{2 c} \frac{Z_{S}^{\prime}(c)}{Z_{S}(c)}-\frac{g-1-\Delta n_{0}^{(0)}}{c^{2}}\right] d c^{2} } \\
= & -\frac{1}{\sqrt{4 \pi t}} \int_{0}^{\infty} u \epsilon^{-u^{2} / 4 t}\left(\mathcal{L}^{-1} \ln Z_{S}\right)(u) d u \cdots\left(g-1+\Delta n_{0}^{(0)}\right)
\end{aligned}
$$

where $\mathcal{L}^{-1}$ denotes the inverse Laplace transformation. In particular this gives

$$
\hat{\Theta}(0)=-\left(g-1+\Delta n_{0}^{(0)}\right)
$$

this result is consistent with equation (14). Equation (14) gives also that for $t \rightarrow \infty$ the supertrace for the heat-kernel for $\square_{m}^{2}$ diverges according to $(m: N)$

$$
\Theta_{m}(t)=\operatorname{str}^{\prime}\left(\exp ^{\prime}\left(t \square_{m}^{2}\right) \simeq(1-g) \epsilon^{m^{2} t}, \quad(t \rightarrow \infty)\right.
$$

(and similar for negative integers), a result found by Aoki 3 .

\section{Discussion of the Spectrum}

The operator $\hat{\square}$ is simpler to study than the operator $\square$, because the trivial contribution $\frac{m}{2}$ to the Eigenvalues has been subtracted (see Eq.(III.49) for the unitary equivalence between $\square_{m}$ and $\hat{\square}_{m}+\frac{m}{2}$ ).

For applying the supertrace formula for the operator $\hat{\Pi}_{m}$ the formulas must be changed appropriately - see Eqs.(IV.34):

$$
\begin{aligned}
A_{0}^{(m)} & =(1-g) \int_{0}^{\infty} \frac{g(u)-g(-u)}{\sinh \frac{u}{2}} T_{m}\left(\cosh \frac{u}{2}\right) d u \\
G(u, \chi) & =g(u)+g(-u)-\chi\left[g(u) \epsilon^{-\frac{u}{2}}+g(-u) e^{\frac{u}{2}}\right],
\end{aligned}
$$

where

$$
g(u)=\frac{1}{2 \pi} \int_{-\infty}^{\infty} \epsilon^{-i u p} h\left(i p+\frac{1}{2}\right) d p .
$$

Thus the function $h$ has taken on the argument $i p+\frac{1}{2}$. This gives immediately:

$$
\begin{aligned}
g(u) & =\frac{1}{\sqrt{4 \pi t}} \epsilon^{-u^{2} / 4 t-c^{2} t+\frac{u}{2}} \\
G(u, x) & =\frac{1}{\sqrt{\pi t}} \epsilon^{-u^{2} / 4 t-c^{2} t}\left(\cosh \frac{u}{2}-\chi\right) \\
A_{0}^{(m)} & =\frac{1-g}{\sqrt{\pi t}} \epsilon^{-c^{2} t} \int_{0}^{\infty} \epsilon^{-u^{2} / 4 t} \cosh m \frac{u}{2} d u \\
& =(1-g) \varepsilon^{-\left(c^{2}-\frac{m^{2}}{4}\right) t},
\end{aligned}
$$

where in the last expression the integral (9) has been used. Therefore we get for the supertrace formula for the resolvent of $\hat{\square}_{m}^{2}$ for $m$ even ("hatted" quantities belonging to $\hat{\mathrm{D})}$ :

$$
\sum_{n=1}^{\infty}\left[\frac{1}{c^{2}-\left(\hat{\lambda}_{n, m}^{B}\right)^{2}}-\frac{1}{c^{2}-\left(\hat{\lambda}_{n, m}^{F}\right)^{2}}\right]=\frac{1-g}{\left(c-\frac{m}{2}\right)\left(c+\frac{m}{2}\right)}+\frac{1}{2 c} \frac{d}{d c} \ln \left[\frac{Z_{0}(c+1) Z_{0}(c)}{Z_{1}^{2}\left(c+\frac{1}{2}\right)}\right]
$$

and similarly for $m$ odd:

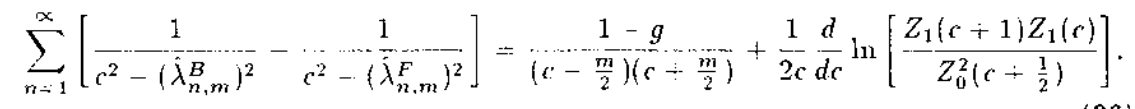

Analysing for the particular values $c=\epsilon$ and $c= \pm \frac{m}{2}+\epsilon$ this gives for $m$ even $(\epsilon) 1)$

$$
\begin{aligned}
\frac{1}{2 c} \frac{d}{d c} \ln \left[\frac{Z_{0}(c+1) Z_{\mathrm{u}}(c)}{Z_{1}^{2}\left(c+\frac{1}{2}\right)}\right] \cdots & \frac{g-1}{\left(c-\frac{m}{2}\right)\left(c+\frac{m}{2}\right)} \\
& \propto \begin{cases}\frac{\Delta \hat{n}_{0}^{(0)}}{\epsilon^{2}} & (c=\epsilon \cdot m=0) . \\
\pm \frac{g-1+\Delta \hat{n}_{0}^{(0)}}{\epsilon^{2}} & (c=\epsilon, m \neq 0) . \\
\pm \frac{1-g}{m \epsilon} & \left(c=-\frac{m}{2}-\epsilon, m \neq 0\right)\end{cases}
\end{aligned}
$$


and regularly otherwise up to the nontrivial zeros and poles of $Z_{0}$ and $Z_{1}$. For $m$ odd for $c= \pm \frac{1}{2}+\epsilon$ and $c= \pm \frac{m}{2}+\epsilon(|\epsilon| \ll 1)$

$$
\begin{aligned}
& \frac{1}{2 c} \frac{d}{d c} \ln \left[\frac{Z_{1}(c+1) Z_{1}(c)}{Z_{0}^{2}\left(c+\frac{1}{2}\right)}\right]-\frac{g-1}{\left(c \cdots \frac{m}{2}\right)\left(c+\frac{m}{2}\right)} \\
& \propto \begin{cases}\mp \frac{g-1+\Delta \hat{n}_{0}^{(0)}}{\epsilon} & \left(c= \pm \frac{1}{2}+\epsilon, m=1\right), \\
\mp \frac{\Delta \hat{n}_{0}^{(0)}}{\epsilon} & \left(c= \pm \frac{1}{2}: \epsilon, m \neq 1\right), \\
\pm \frac{1-g}{m \epsilon} & \left(c= \pm \frac{m}{2}+\epsilon, m \neq 1\right)\end{cases}
\end{aligned}
$$

and regularly otherwise up to the nontrivial zeros and poles of $Z_{0}$ and $Z_{1}$. Let us discuss two scenarios for $\Delta \hat{n}_{0}^{(0)}$ :

1) $\Delta \hat{n}_{0}^{(0)}=0$ : This yields for the various trivial modes of $\widehat{\mathrm{C}}_{m}$ for $m$ even:

$$
\begin{array}{llll}
\underline{m \text { even: }} & \frac{m \text { odd: }}{(1)} \\
\Delta \hat{n}_{0}^{(\theta)}=0 & & \Delta \hat{n}_{0}^{(1)}=g-1 & \\
\Delta \hat{n}_{0}^{(m)}=g-1 & (m \neq 0), & \Delta \hat{n}_{ \pm \frac{1}{2}}^{(m)}=0 & (m \neq 1), \\
\Delta \hat{n}_{ \pm \frac{m}{2}}^{(m)}=1 \cdots g & (m \neq 0) ; & \Delta \hat{n}_{ \pm \frac{m}{2}}^{(m)}=1-g & (m \neq 1) .
\end{array}
$$

2) $\Delta \hat{n}_{0}^{(0)}=1-g$ : In a similar way:

$$
\begin{aligned}
& \text { m even: } \\
& m \text { odd: } \\
& \Delta \hat{n}_{0}^{(0)}=1-g \\
& \Delta \hat{n}_{0}^{(1)}=0 \\
& \Delta \hat{n}_{0}^{(m)}=0 \\
& (m \neq 0), \\
& \Delta \hat{n}_{ \pm \frac{1}{2}}^{(m)}=g-1 \\
& (m \neq 1) \text {, } \\
& \Delta \hat{n}_{ \pm \frac{m}{2}}^{(m)}=1-g \\
& (m \neq 0) \text {; } \\
& \Delta \hat{n}_{ \pm \frac{m}{2}}^{(m)}=1-g
\end{aligned}
$$

That trivial-modes or trivial Eigenvalues (as the trivial-modes of $\square_{m}$ ) appear can be understood in the view of the corresponding results for the classical Laplacian $-\Delta_{m}$ as discussed, e.g. by Hejhal $\left[49\right.$, p.408]. Let $\left\{\lambda_{n}^{(m)}\right\}$ be the set of all Eigenvalues of the Laplace-operator $-\Delta_{m}=-y^{2}\left(\partial_{x}^{2}+\partial_{y}^{2}\right)+i m y \partial_{x}$ and $m \geq 2$. Then $(n \in \mathbf{N})$ :

$$
\left\{\lambda_{n}^{(m)}\right\}=\left\{\frac{m}{2}\left(1-\frac{m}{2}\right)\right\}_{k=1}^{d} \cup\left\{\lambda_{n}^{(m-2)} \mid \lambda_{n}^{(m-2)} \neq \frac{m}{2}\left(1-\frac{m}{2}\right)\right\},
$$

where $d=\delta+(g-1)(m-1)$ and $\delta$ takes on the values 0 and 1 , depending on $m$. There are several methods of obtaining this result. E.g. one can first consider the Selberg trace formula for the (regularized) resolvent-kernel function and deduces this statement from the analytical properties of the Selberg zeta-function (nontrivial Eigenvalues) and the poles occuring in the $A_{0}$ term (trivial-modes); second, one can consider commutation relations of the differential operators $\nabla_{z}^{k}$ acting on tensorfields which give simple recursion formulas for the Laplacian $\Delta_{m}$ depending on the curvature $R$ of the space in question [16].
Equations (35) and (36) give also the relation of the Eigenvalues $\lambda_{n, m}$ of $\hat{\varphi}_{m}$ and $\hat{\lambda}_{n}$ of $\hat{\square}_{0}$. Due to the analytic structure of the super zeta-functions for the nontrivial Eigenvalues this gives:

$$
\dot{\lambda}_{n, m}=\dot{\lambda}_{n}, \quad(n \in \mathbf{N}, m \in \mathbf{N}) .
$$

This simple result corresponds again to the classical one noted in $\mathrm{Eq} .(39)$.

\section{Determinants and the Fermionic String Integrand}

The starting points for the calculation of determinants of the operator $\square_{m}^{2}$ are Eqs.(23-26) which all can be analytically continued to $c=0$ (including a omission of zero-modes if necessarry). Let us first consider Eq.(23) for $m=0$. Performing the limit $c \rightarrow \epsilon$ for $|\epsilon| \ll 1$

$$
\operatorname{sdet}\left(-\square_{0}^{2}\right)=\frac{1}{(2 g-2) !} \cdot \frac{Z_{0}(1) Z_{0}^{(2 g-2)}(0)}{\left[\bar{Z}_{1}\left(\frac{1}{2}\right)\right]^{2}} \epsilon^{2 \Delta n_{0}^{(0)}} .
$$

Here by $\dot{Z}_{1}\left(\frac{1}{2}\right)$ the appropriate derivative or residuum of $Z_{1}$ at $s=\frac{1}{2}$ is denoted, depending whether $\Delta n_{0}^{(0)} \leq 0$ or $\Delta n_{0}^{(0)}>0$, respectively. To make this quantity well-defined I subtract from sdet $\left(-\square_{0}^{2}\right)$ the zero-mode which I denote by priming the sdet. Using further the functional relation (V.42) for $Z_{0}$ and $Z_{1}$ I get finally:

$$
\operatorname{sdet}^{\prime}\left(-\square_{0}^{2}\right)=\left[\pi^{g-1} \frac{Z_{0}(1)}{\tilde{Z}_{3}\left(\frac{1}{2}\right)}\right]^{2} \frac{Z_{1}(0)}{Z_{1}(1)}
$$

For calculating the superdeterminant for $m$ even and $m \geq 2$ a subtraction of zeroor trivial-modes is not necessary. Proceeding similarly as for $m=0$ we get for $m-2,4 \ldots$ :

$$
\operatorname{sdet}\left(-\square_{m}^{2}\right)=\left[\left(\frac{\pi}{m !}\right)^{g-1} \frac{Z_{0}\left(1+\frac{m}{2}\right)}{Z_{1}\left(\frac{m+1}{2}\right)}\right]^{2} \frac{Z_{1}(0)}{Z_{1}(1)} .
$$

Similarly $(m=2,4 \ldots)$ :

$$
\operatorname{sdet}\left(-\square_{-m}^{2}\right)=\left[\left(\frac{(m-2) !}{\pi}\right)^{g-1} \frac{Z_{0}\left(\frac{m}{2}\right)}{Z_{1}\left(\frac{m+\frac{1}{2}}{2}\right)}\right]^{2} \frac{Z_{3}(1)}{Z_{1}(0)} .
$$

For $m=1,3, \ldots$ :

$$
\operatorname{sdet}\left(\cdot \square_{m}^{2}\right)=\left[\left(-\frac{\pi}{i m !}\right)^{g-1} \frac{Z_{1}\left(1+\frac{m}{2}\right)}{Z_{0}\left(\frac{m !+1}{2}\right)}\right]^{2} \frac{Z_{1}(0)}{Z_{1}(1)}
$$

and $m=3,5, \ldots$ :

$$
\operatorname{sdet}\left(-\square_{-m}^{2}\right)=\left[\left(\frac{(m-2) ! i}{\pi}\right)^{g-1} \frac{Z_{1}\left(\frac{m}{2}\right)}{Z_{0}\left(\frac{m+1}{2}\right)}\right]^{2} \frac{Z_{1}(1)}{Z_{1}(0)} .
$$

Note the differences to Ref.|3| which are due to the additional super zeta-functions. The case of $\square_{-1}^{2}$ must be treated separately because of the appearence of zero-modes 
which must be subtracted. Therefore denoting the omission of zero-modes by priming the super determinant I get

$$
\operatorname{sdet}^{\prime}\left(-\square_{-1}^{2}\right)=\left[\pi^{1-g} \frac{\tilde{Z}_{1}\left(\frac{1}{2}\right)}{Z_{0}(1)}\right]^{2} \frac{Z_{1}(1)}{Z_{1}(0)}
$$

From the introduction we know that the relevant string integrand is given by $\operatorname{sdet}^{\prime}\left(-\square_{0}^{2}\right)$ and $\operatorname{sdet}\left(-\square_{2}^{2}\right)$. Equations (42) and (43) yield:

$$
\begin{aligned}
& {\left[\operatorname{sdet}^{\prime}\left(-\square_{0}^{2}\right)\right]^{-\frac{5}{2}}\left[\operatorname{sdet}\left(-\square_{2}^{2}\right)\right]^{\frac{1}{2}}} \\
& \quad=\left(\pi^{g-1} \frac{Z_{0}(1)}{\bar{Z}_{1}\left(\frac{1}{2}\right)}\right)^{-5}\left(\frac{\pi}{2}\right)^{g-1} \frac{Z_{0}(2)}{Z_{1}\left(\frac{3}{2}\right)}\left(\frac{Z_{1}(1)}{Z_{1}(0)}\right)^{2}
\end{aligned}
$$

or alternatively

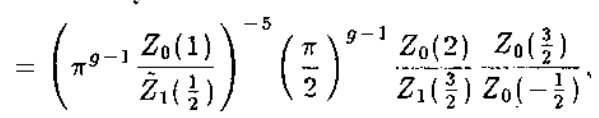

and we can conclude that this expression is well defined. Furthermore for $Z_{g}$ of Eq. (I.38):

$$
\begin{aligned}
Z_{g}=\int_{s \mathcal{M}_{g}} d(S W P)\left[\operatorname{sidet}^{\prime}\left(-\square_{0}^{2}\right)\right]^{-\frac{5}{2}}\left[\operatorname{sdet}^{\prime}\left(\cdots \square_{2}^{2}\right)\right]^{\frac{3}{2}} \\
=\left(\frac{1}{2 \pi^{4}}\right)^{g-1} \int_{s \mathcal{M}_{g}} d(S W P)\left(\frac{Z_{0}(1)}{\bar{Z}_{1}\left(\frac{1}{2}\right)}\right)^{-5} \frac{Z_{0}(2)}{Z_{1}\left(\frac{3}{2}\right)}\left(\frac{Z_{1}(1)}{Z_{1}(0)}\right)^{2} .
\end{aligned}
$$

This is the main result of this section. Note the appearence of the various ratios of the Selberg super zeta-functions. The main difference to Aoki $[3]$ who first calculated super determinants of Laplace-Dirac operators lies in the additional factor $\left[Z_{1}(1) / Z_{1}(0)\right\}^{2}$ in the superdeterminants. This factor is unambigously given by the functional equations which have been used to derive $\mathrm{Eq} .(48)$ and it changes the supermoduli dependence of the integrand. In the result of Ref.[3] this factor did not appear because the functional relations of the Selberg super zeta-functions were not available.

Unfortunately no statement. like the analysis of Gross and Periwal 45 ! about the growing properties of this expression for increasing genus $g$ can be made because an expansion in the super noduli gives unknown signs in the soul-contributions of the Selberg super zeta-functions, Gross and Periwal found for the relevant terms in the genus-expansion of the Polyakor partition function $Z$ for the bosonic string

$$
\begin{aligned}
& Z=\sum_{g=0}^{x} Z_{g} \\
& Z_{g}=\int_{M_{s}} d(W P) \operatorname{det}\left(\Delta_{0}\right)^{-13} \operatorname{det}\left(\Delta_{i}\right)
\end{aligned}
$$

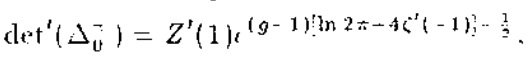

$$
\begin{aligned}
& \operatorname{det}\left(\Delta_{1}\right)=Z(2) e^{(g-1): 3 \ln 2 \pi \cdot 1 \zeta^{\prime}(\cdots) \cdot \frac{v^{2}}{2}}, \\
& Z^{\prime}(1) \cdots C_{c} B_{\epsilon}^{4 \pi(g \cdots 1)} \exp \left\{-\int_{c}^{\infty}{ }_{t}^{d t} \Theta(t) \cdot 1\right\} \\
& Z(2) \quad Z(1+\delta) \cdot Z^{\prime}\left(1 \mid K(\delta, \epsilon)^{4 \pi+J^{-1}} U(\delta, \epsilon)\right.
\end{aligned}
$$

where $d(W P)$ is the Weil-Peterson measure, $M_{g}$ the moduli space, $Z$ the usual Selberg zeta-function, $\Theta$ the trace of the heat-kernel on compact domains on the Poincaré upper half-plane ars $C_{\epsilon}, B_{\epsilon}, K^{\prime}(\delta, \epsilon)$ and $M(\delta, \epsilon)$ are constants independent of the genu. g. From Eq.(50) one finds that $Z^{\prime}(1)^{-1}$ and $Z(2)$ have an exponential growth for in creasing genus; together with the properties of the Weil-Peterson measure, i.e. that it volume increases factorial-like for increasing genus one concludes that the pertubation series in $g$ for the partition function $Z$ diverges.

Nevertheless we can discuss the behaviour of the fermionic string integrand for the case of degenerate super Riemann surfaces. For this purpose let us consider such a surface, i.e. a pinching process takes place and at least the lenght of one geodesic vanishes. Let $l_{0}$ be the geodesics of $\gamma_{0} \in \Gamma$ with $\left(l_{0}\right)_{\text {Body }}<\left(l_{\gamma}\right)_{\text {Body }}$ for all $\gamma \in \Gamma$ with $\gamma \neq \gamma_{0}$. Let us introduce the partial zeta-functions $\mathcal{Z}_{q}(s)=\mathcal{Z}_{q}\left(s, l_{0}\right)$ with

$$
\mathcal{Z}_{q}(s):=\prod_{n=0}^{\infty}\left[1-x_{\gamma o}^{q} \epsilon^{-\{s+n) l_{0}} ;, \quad(q=0.1, \operatorname{Re}(s\}>1) .\right.
$$

For the entire zeta-functions one has

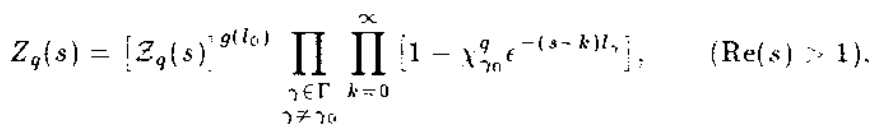

where $g\left(I_{0}\right)$ denotes the multiplicity of $l_{0}$. A discussion for the bosonic string is due to Wolpert 95 who showed that for $l_{0} \rightarrow 0$ one has (set $q=0$ in Eq.(51) and interpret all quantities in terms of the bosonic case)

$$
\mathcal{Z}(s) \times l_{0}^{\frac{1}{2}-s} \exp \left(-\frac{\pi^{2}}{6 l_{0}}+O\left(l_{0}\right)\right), \quad\left(l_{0} \rightarrow 0\right) .
$$

This asymptotic behaviour has the immediate consequence that the bosonic string has a divergence due to geodesics of zero-length. This result can be generalised to the fermionic string! To see this let us start by taking the logarithm of partial zetafunction:

$$
\begin{aligned}
& -\ln Z_{q}(s)=-\sum_{n=0}^{\infty} \ln \left(1-x_{\gamma_{i+1}}^{q} \cdot(s-n) l_{i i}\right) \\
& =\sum_{n=0}^{\infty} \sum_{k=1}^{\infty} \frac{1}{k} l_{r 0}^{k} \epsilon^{-k(n+n) h_{0}}=\sum_{k=1}^{\infty} \frac{k_{k+1}^{k} e^{-k \cdot k l_{0}}}{k\left(1-t^{-k l_{0}}\right)}
\end{aligned}
$$

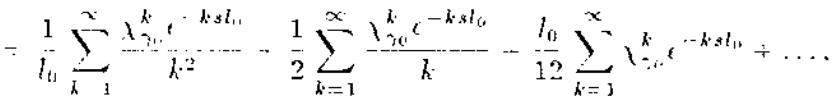

where the denominator was expanded as

$$
1-\frac{1}{2} \cdots l_{u}-\frac{1}{k l_{0}}-\frac{1}{2}-\frac{k l_{0}}{12}-O\left(k^{2} l_{i}^{2}\right)
$$

For the varions sums one gets

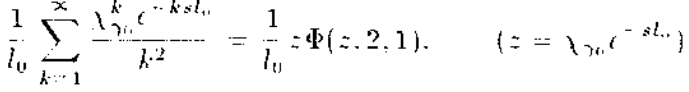

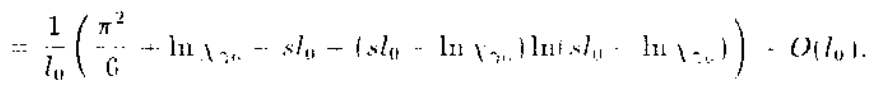


where $\Phi(z, s, a):=\sum_{n=0}^{\infty} \frac{z^{n}}{(a+n)^{2}}$ is Lerch's transcendent 55, p. 32: With the expansion

$\Phi(z, m, a)=z^{-a}\left\{\sum_{\substack{n=0 \\ n \neq m-1}}^{\infty} \zeta(m-n, a) \frac{(\ln z)^{n}}{n !}+\frac{(\ln z)^{m-1}}{(m-1) !}\left[\Psi(m)-\Psi(a)-\ln \left(\ln \frac{1}{z}\right)\right]\right\}$

(note $\zeta(s, 1)=\zeta(s), \zeta(2)=\pi^{2} / 6$ and $\left.\Psi(2)-\Psi(1)=1\right)$. Furthermore

$$
\begin{aligned}
\frac{1}{2} \sum_{k=1}^{\infty} \frac{\chi_{\gamma_{0}}^{k} \epsilon^{-k s l_{0}}}{k} & =-\frac{1}{2} \ln \left(1-\chi_{\gamma 0} \epsilon^{-s l_{0}}\right) \\
& =-\frac{1}{2} \ln \left(s l_{0}\right)+O\left(l_{0}\right), \quad\left(\chi_{\gamma_{0}}=1\right), \\
\frac{l_{0}}{12} \sum_{k=1}^{\infty} \chi_{\gamma_{0}}^{k} \epsilon^{-s k l_{0}} & =\frac{l_{0}}{12} \frac{\chi_{\gamma 0} \epsilon^{-s l_{0}}}{1-\chi_{\gamma 0} \epsilon^{-s l_{0}}} \\
& =\frac{1}{s l_{0}}-\frac{1}{2}+O\left(l_{0}\right), \quad\left(\chi_{70}:=1\right) .
\end{aligned}
$$

The last two expansions are valid for $\left(s l_{0}\right)_{B o d y}>0$. Furthermore no singularities appear for $\chi_{\gamma_{0}}=-1$. This gives in the case of $\chi_{\gamma_{0}}=1$ the expansion for $\left(-\ln \mathcal{Z}_{q}\right)$ :

$$
-\ln \mathcal{Z}_{0}(s)=\frac{\pi^{2}}{6 l_{0}}+\left(s-\frac{1}{2}\right) \ln l_{0}+\text { const. }+O\left(l_{0}\right)
$$

which is equivalent with Eq.(54). For $\chi_{\gamma_{0}}=-1$ things are changed and we get:

$$
\begin{aligned}
& -\left.\ln \mathcal{Z}_{1}(s)\right|_{\lambda_{\gamma_{0}}=-1} \\
& \quad=\frac{1}{l_{0}}\left(\frac{\pi^{2}}{6}+i \pi\right)-\frac{i \pi}{l_{0}} \ln \left(l_{0}-\frac{i \pi}{s}\right)+s \ln \left(l_{0}-\frac{i \pi}{s}\right)+\text { const. }+O\left(l_{0}\right) .
\end{aligned}
$$

Therefore we have to descriminate between $x_{\gamma 0}=1$ and $x_{70}=\cdots 1$. Let us first assume that the character $\chi_{\gamma o}$ corresponding to the smallest geodesic is positive or that this can be achieved by an appropriate redefinition of the $4 g$ generators $\gamma_{i}, \uparrow_{i}^{-1}$ $(i=1, \ldots, 2 g)$. In the relevant combinations for sdet $\left(-\square_{m}^{2}\right)$ this gives:

$$
\begin{aligned}
& \frac{\mathcal{Z}_{0}\left(s-\frac{m}{2}\right) \mathcal{Z}_{0}\left(s+1+\frac{m}{2}\right)}{\mathcal{Z}_{1}\left(s+\frac{m+1}{2}\right) \mathcal{Z}_{1}\left(s+\frac{1-m}{2}\right)} \propto \text { const. }, \quad\left(m \in \mathbf{Z}, \text { even, } l_{0} \ldots, 0\right), \\
& \frac{\mathcal{Z}_{1}\left(s-\frac{m}{2}\right) \mathcal{Z}_{1}\left(s+1+\frac{m}{2}\right)}{\mathcal{Z}_{0}\left(s+\frac{m+1}{2}\right) \mathcal{Z}_{0}\left(s+\frac{1-m}{2}\right)} \times \text { const. }, \quad\left(m \in \mathbf{Z} \text {. odd. } l_{u} \rightarrow 0\right),
\end{aligned}
$$

where the const. may depend on $s$. Therefore in this case the determinants are proportional to a constant $(m \in \mathbf{Z})$ and thus the fernionic string integrand is finite.

In the case of $\chi_{\gamma_{0}}=-1$ things are changed and we get in the limit $l_{0} \rightarrow 0$, e.g. for $m=0$

$$
\begin{aligned}
& \frac{\mathcal{Z}_{0}(s) \mathcal{Z}_{0}(s+1+)}{\mathcal{Z}_{1}^{2}\left(s+\frac{1}{2}\right)} \propto e^{\frac{2 i \pi}{l_{0}} l_{0}^{-2 s}\left(l_{0}-\frac{i \pi}{s+\frac{1}{2}}\right)^{2 s-1-\frac{2 i \pi}{l_{0}}}} \\
& \frac{\mathcal{Z}_{1}(s) \mathcal{Z}_{1}(s+1)}{\mathcal{Z}_{0}^{2}\left(s+\frac{1}{2}\right)} \propto e^{\frac{-2 i \pi}{l_{0}}} l_{0}^{2 s}\left(l_{0}-\frac{i \pi}{s}\right)^{\frac{i \pi}{i_{0}}-s}\left(l_{0}-\frac{i \pi}{s+1}\right)^{\frac{i \pi}{i_{0}}-s-1}
\end{aligned}
$$

where again the const. may dcpend on $s$. In this case the fermionic string integrand diverges for $l_{0} \rightarrow 0$ as in the bosonic case.

Finally let us consider the product of the superdeterminants of $-\square_{0}^{2}$ and $-\square_{-1}^{2}$ :

$$
\operatorname{sdet}^{\prime}\left(-\square_{0}^{2}\right) \cdot \operatorname{sdet}^{\prime}\left(-\square_{\cdots 1}^{2}\right)=1
$$

which follows directly from Eqs.(42) and (4i). Generalizing this interesting result we find (omitting zero-modes if necessary):

$$
\operatorname{sdet}^{\prime}\left(-\square_{-m}^{2}\right) \cdot \operatorname{sdet}^{\prime}\left(-\square_{m-1}^{2}\right)=(-1)^{g-1}(m-1)^{2-2 g}, \quad(m \in \mathbf{Z}) .
$$

Let be $f_{m}=\left[I_{k 0}^{m}\left(\cdot k^{2}\right)^{g-1}\right.$. Redefining the superdeterminant by $\operatorname{set}^{\prime}\left(-\square_{m}^{2}\right):=$ $\left.\operatorname{sdet}^{\prime}(-\square]_{m}^{2}\right) / f_{m}(m \geq 0)$ and $\operatorname{sidet}^{\prime}\left(-\square_{-m}^{2}\right):=\operatorname{sdet}^{\prime}\left(-\square_{-m}^{2}\right) \cdot f_{m-2}(m \geq 1)$ this gives the relation

$$
\operatorname{sdet}^{\prime}\left(\square_{-m}^{2}\right) \cdot \operatorname{sdet}^{\prime}\left(-\square_{m-1}^{2}\right)=1, \quad(m \in \mathbf{Z}) .
$$

An equation like this was already stated by Baranov and Schwarz 11 by more general considerations. I close with this result, which nicely confirms my own considerations. 


\section{SUMMARY}

In this paper I have discussed the Selberg supertrace formula on super Riemann surfaces and some of its most important consequences. In chapter II a short survey of super analysis and super manifolds was given. Fitted with that knowledge it was possible to study in chapter III physics on the super Poincare upper half-plane, i.e. classical and quantum motion. Starting from the Vielbein approach a well-behaving nonsingular metric on $S \mathcal{H}$ was constructed. From this metric the classical Lagrangian and Hamiltonian could be easily constructed. Also, the construction of the quantum Hamiltonian has been straightforward and was defined by the super Laplace-Beltrami operator $\Delta_{S L B}$ on $S \mathcal{H}$. The path integral on $S \mathcal{H}$ was written down and proved in Appendix B to be the correct one. Surprisingly enough this super Laplace-Beltrami operator could be factorized into the square of the Laplace-Dirac operator: $\Delta_{S L B}=\square^{2}=(2 Y D \bar{D})^{2}$. Also the more general operators $\hat{\square}_{m}$ and $\square_{m}$ were introduced which are connected by a linear isomorphism.

With chapter IV the main part of this paper started. The Selberg super operator $L$ on $S \mathcal{H}$ was defined and it was found that the operator $L$ multiplies an arbitrary Eigenfunction of $\square_{m}$ by the function $h$, where $h$ is only defined by the Eigenvalue $s$ of this Eigenfunction with respect to $\square_{m}$ and the integral kernel of $L$. The calculation yielded that for appropriate $h$ the operator $h(\square m)$ equals to an integral operator $L$ whose integral kernel $k_{m}\left(Z, W^{\prime}\right)$ is related to $h$ by the equations

$$
\begin{aligned}
h_{m}(s) & =\int_{-\infty}^{\infty} d u e^{u\left(s-\frac{1}{2}\right)} g(u), \quad\left(s=\frac{1}{2}+i p\right), \\
g(u) & =\frac{1}{2 \pi} \int_{-\infty}^{\infty} d p e^{-i u p} h_{m}\left(\frac{1}{2}+i p\right) \\
g(u) & =\frac{1}{8} \int_{4 \sinh ^{2} \frac{u}{2}}^{\infty} \frac{d x}{(x+4)^{\frac{m}{2}}} \\
& \times\left[\frac{\Psi(x)+2\left(\epsilon^{u}-1\right) \Phi(x)}{\sqrt{x \cdots 4 \sinh ^{2} \frac{u}{2}}}\left(a_{+}^{m}-\alpha_{-}^{m}\right)-i m \epsilon^{\frac{u}{2}} \Phi(x) \frac{a_{+}^{m}-a_{-}^{m}}{x+4}\right] .
\end{aligned}
$$

It was found that the Selberg supertrace formula reads

$$
\begin{aligned}
& \sum_{n=0}^{\infty}\left[h_{m}\left(p_{n}^{B}\right)-h_{m}\left(p_{n}^{F}\right)\right\rfloor=(1-g) \int_{0}^{\infty} \frac{g(u)-g(-u)}{\sinh \frac{u}{2}} \cosh \frac{m}{2} d u
\end{aligned}
$$

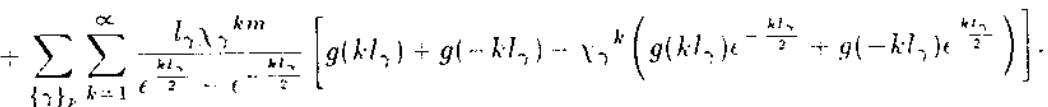

The inversion formula which is needed in the supertrace formula to calculate the tem $A_{0}^{(m)}=i^{m} \pi(g-1) \Phi_{m}(0)$ which corresponds to the identity transformation was found to be given by

$$
i^{m} \Phi_{m}(x)=\frac{1}{\pi \sqrt{x^{2}+4}} \int_{x}^{\infty} \frac{d y}{\sqrt{y+4}} \int_{-\infty}^{\infty} Q^{\prime}\left(y+t^{2}\right)\left(\frac{1 y-4+t^{2}-t}{\sqrt{y-4}-t t^{2}+t}\right)^{\frac{m}{2}} d t .
$$

where $\left.Q(u)=2 \operatorname{coth} \frac{u}{2} g(u)-g(-u)\right] . A_{0}^{(m)}$ and the inversion formula for $\Phi_{m}(0)$ completed the work of Baranov et al. 10.11 by explicitly stating the $A_{u}^{(m)}$-term and the inversion formula. respectively.
Chapter $\mathrm{V}$ was devoted to the discussion of the analytic properties of the two Selberg super zeta-functions $Z_{0}$ and $Z_{1}$ :

$$
\begin{aligned}
& Z_{0}(s)=\prod_{\gamma \in \Gamma} \prod_{k=0}^{\infty}\left[1-\epsilon^{-(s+k) l_{\gamma}}\right], \quad(\operatorname{Re}(s)>1), \\
& Z_{1}(s)=\prod_{\gamma \in \Gamma} \prod_{k=0}^{\infty}\left[1-x_{\gamma} e^{-(s+k) l_{\gamma}}\right], \quad(\operatorname{Re}(s)>1) .
\end{aligned}
$$

By considering specific test functions for the analytic properties of $Z_{1}\left(k \in \mathbf{N}_{0}\right)$ could be derived:

- $: s=\frac{1}{2}$ : There are a pole, zero with multiplicity $\left|\Delta n_{0}^{(0)}\right|$, respectively, or a regular point depending on whether $\Delta n_{0}^{(0)}>0, \Delta n_{0}^{(0)}<0$ or $\Delta n_{0}^{(0)}=0$.

- $: s=-\frac{1}{2}-k$ : There are zeros with multiplicity $4(k+1)(g-1)$.

- : $s=i p_{n}^{B, F}-k$ : There are zeros $\left(p_{N}^{B}\right)$ and poles $\left(p_{n}^{F}\right)$;

- : $s=-i p_{n}^{B, F}-k$ : There are poles $\left(p_{N}^{B}\right)$ and $\operatorname{zeros}\left(p_{n}^{F}\right)$

with the same multiplicity as the corresponding Eigenvalue of $\square$ : respectively. The crucial importance of $\Delta n_{0}^{(0)}=$ \#ercn $(z e r o-\operatorname{modes})-$ \#odd(zero-modes $)$ of the operator $\square$ has become clear. A functional relation for $R_{1}$ and $Z_{1}$, respectively, was derived:

$$
R_{1}(s) R_{1}(-s)=\frac{Z_{1}(s) Z_{1}(-s)}{Z_{1}(s+1) Z_{1}(1-s)}=\frac{Z_{1}^{2}(0)}{Z_{1}(1)}(\cos \pi s)^{4(g-1)} .
$$

Similarly for $Z_{0}\left(k \in \mathbf{N}_{0}\right)$

- : $s=-k$ : There are trivial zeros with multiplicity $(g-1)(4 k+2)$.

- $: s=\frac{3}{2}+i p_{n}^{B, F}-k$ : There are zeros $\left(p_{n}^{B}\right)$ and poles $\left(p_{n}^{F}\right)$

- : $s:=-\frac{1}{2}-i p_{n}^{B, F}-k$ : There are poles $\left(p_{n}^{B}\right)$ and zeros $\left(p_{n}^{F}\right)$,

of course. with the same multiplicity as the corresponding Eigenvalues of $\square$, respectively. It was found that there is no zero of $Z_{0}(s)$ at $s=1$. This is quite different in comparision to the usual Selberg zeta-function.

Also a functional equation for $R_{0}$ and $Z_{0}$, respectively, was derived reading

$$
R_{0}(s) R_{0}(-s)=\frac{Z_{0}(s) Z_{0}(-s)}{Z_{0}(s-1) Z_{0}(1 \cdots s)}=\frac{Z_{0}\left(\cdots \frac{1}{2}\right)}{Z_{0}\left(\frac{3}{2}\right)}(\sin \pi s)^{4(g-1)} .
$$

For both functions we get an infinite set of critical lines located for $Z_{0}$ at $\operatorname{Re}(s)=\frac{1}{2}-k$ $\left(k \in \mathrm{N}_{0}\right)$ and for $Z_{1}$ at $\operatorname{Re}(s)=-k \cdot\left(k \in \mathrm{N}_{0}\right)$.

Unfortunately no functional equation for $Z_{0}$ or $Z_{1}$ as for the ordinary Selberg zetafunction could be found. However. I have argued the unlikelihood that such a relation exists, based on the existence of the infinite nuntier of critical lines.

This appearence of an infinite number of critical lines for the two functions $Z_{0}$ and $Z_{1}$ is surprising, because there is not any classical analugy for this feature. However. in view of the functional relations for $R_{0}\left(Z_{0}\right)$ and $R_{1}\left(Z_{3}\right)$ this is a consistent result The functional relations are not of any use for the determination of the spectrum of the Laplace-Dirac operator $\square$. This in turn is the same situation as in the clasical 
case. There is up to now no way into the critical domain of the (super) zeta-functions in the complex plane, where the nontrivial zeros (and/or poles) are located.

By an appropriate test function $h$ a functional relation connecting $Z_{0}$ and $Z_{1}$ conld be derived:

$$
\frac{Z_{1}\left(\frac{1}{2} \cdots s\right) Z_{0}(s)}{Z_{1}\left(\frac{1}{2}+s\right) Z_{0}(1-s)}=\frac{Z_{1}(0)}{Z_{1}(1)}(\sin \pi s)^{2(g-1)}
$$

A relation like this was already stated by Baranov and Schwarz 11: but without the chararteristic $(\sin \pi s)^{2 g-2}$-dependence.

Having discussed the properties of $Z_{0}$ and $Z_{1}$ I treated in the final chapter the spectrum and superdeterminants of the Laplacian-Dirac operators $\hat{\square}_{m}$ and $\square_{m}$, respectively. Denoting by $\Delta \hat{n}_{\lambda}^{(m)}$ the difference of the even and odd trivial-modes $\lambda$ of the operator $\hat{\square}_{m}$ two scenarios for $\Delta \hat{n}_{0}^{(0)}$, i.e. $\Delta \hat{n}_{0}^{(0)}=0$ and $\Delta \hat{n}_{0}^{(0)}=1-g$. respectively, were discussed.

For the nontrivial Eigenvalues of $\hat{\mathrm{G}}_{m}$ I found that they are determined by the nomtrivial Eigenvalues of $\square_{0}$ as

$$
\hat{\lambda}_{n, m}=\dot{\lambda}_{n} . \quad(n \in \mathbf{N}, m \in \mathbf{N}) .
$$

The calculation of the determinants was performed with the well-known zcta-regularization method. The result for e.g. sdet $^{\prime}\left(-\square_{0}^{2}\right)$ was given by

$$
\operatorname{sdet}^{\prime}\left(-\square_{0}^{2}\right)=\left[\pi^{g-1} \frac{Z_{0}(1)}{\bar{Z}_{1}\left(\frac{1}{2}\right)}\right]^{2} \frac{Z_{1}(0)}{Z_{1}(1)} .
$$

and similarly for sdet' $\left(-\square_{m}^{2}\right)(m \in \mathbf{Z})$. These representations showed clearly that the superdeterminants are well-defined quantities. Since the superdeterminants were well-defined, it could be shown that the fermionic string integrand in the Polyakor approach is well-defined. The remaining integral over the super moduli space reads

$$
\begin{aligned}
Z_{g} & =\int_{s \mathcal{M}_{g}} d(S W P)\left[\operatorname{sdet}^{\prime}\left(-\square_{0}^{2}\right)\right]^{-\frac{5}{2}}\left[\operatorname{sdet}^{\prime}\left(-\square_{2}^{2}\right)\right]^{\frac{1}{2}} \\
& =\left(\frac{1}{2 \pi^{4}}\right)^{g-1} \int_{s \mathcal{M}_{g}} d(S W \cdot P)\left(\frac{Z_{0}(1)}{\tilde{Z}_{1}\left(\frac{1}{2}\right)}\right)^{-5} \frac{Z_{0}(2)}{Z_{1}\left(\frac{3}{2}\right)}\left(\frac{Z_{1}(1)}{Z_{1}(0)}\right)^{2} .
\end{aligned}
$$

Unfortunately no statement about the growing properties of this expression for in creasing genus $g$ like the analysis of Gross and Periwal :45: could be made However, it could discussed what happens for the fermionic string integrand if a pinching takes place. Here a divergence as well as a convergence can happen, depending on the spin structure.

An interesting feature of the determinants is that there is a typical factor of $Z_{1}(0) / Z_{1}(1)$. This factor does not appear in the work of Aoki $33^{1}$, who started from quite analogous expressions but used the functional relation of the ordinary Selberg zeta-function [49] instead of the functional relations for the Selberg super zeta-functions. But this factor is an unambigous consequence of the functional relations which I derived in chapter V. I do not see any way to simplify this charateristic factor any further by exploiting all these functional relations. Therefore this factor gives an additional contribution in the super moduli dependence of the superdeterninants and thus also for the fermionic string integrand.

An interesting relation for the determinants was deduced reading

$$
\operatorname{sdet}^{\prime}\left(-\square_{-m}^{2}\right) \cdot \operatorname{sdet}^{\prime}\left(-\square_{m-1}^{2}\right)=(-1)^{g-1}(m-1)^{2-2 g} . \quad(m \in \mathbf{Z}) .
$$

These results which are all direct cunsequences of the Selberg super trace formula demonstrate in an impressing way the power of the trace formula.

In Appendix E I have given a review of path integration on the Poincare upper half-p]ane $\mathcal{H}$. It is a short summary of recent results in this field obtained by F. Steine and myself $38-43$.

The fact that the fermionic string theory is, formulated in the super analysis formulation, well-defined. is a step forward in the understanding of the whole string theory. However, one must keep in mind that the fermionic string is as well as the bosonic string nothing but a toy-model. To incorporate supersymmetry or to get the standard-model gauge symmetries. the superstring or the heterotic string theory is needed (The higher-loop partition function for the latter has been constructed by Ifoore. Nelson and Polchinski 63). Whereas the incorporation of the superstring can be done by the GSO-projection, it is not obvious to formulate a Selberg trace formula for the heterotic string case and to study its consequences. Again new surprising features may occur.

I think that we must face the possibility that we do not know up to now enough mathematics to understand this new physics, and once again physics may be too hard for the physicists.

\section{Acknowledgements}

I would like to thank Prof. Frank Steiner for his raluable help in completing my Ph.D. Thesis, for many discussions and for fruitful collaboration. I also like to thank him for encouraging me not to forget string theory within all the pat h integrals.

I furthermore thank all my friends and colleagues from which I get received hints. suggestions and the neccessary support: Ralf Aurich. Thilo Berger. Norbert Falck. Dietrich Gaede, ny parents Heinz and Enmy Grosche, Susan Hohl. Andrea Kernemann, Jan McArthur, Gernot Münster, Holger Nimnemann. Andreas Pittelkow, hlaus Pinn. Bernd Raabe, Claudia Renner. Martir Sieber, Hendrik Ticdemann. Hans-Jög Timmze, Hans-Werner Wiesbrock, Gaby Zürn.

Part of this work was supported by "Gradujertenstipendium Lniversität Hamburg". 
Appendix A: Discussion of Invariance Properties of Important Quantimies

1. The Volume Element $d V(Z)$

Let us consider a $S P L(2, \mathbf{R})$ transformation which is following Chapter II given

by

$$
\begin{aligned}
& z^{\prime}=\frac{\delta \theta+a z+b}{\gamma \theta+c z+d} \equiv \frac{A}{B}, \\
& \theta^{\prime}=\frac{e \theta+\alpha z+\beta}{\gamma \theta+c z+d} \equiv \frac{\Gamma}{B},
\end{aligned}
$$

where

$$
\begin{gathered}
A(Z)=a z+b-\theta \delta, B(Z)=c z+d-\theta \gamma, \Gamma(Z)=\alpha z+\beta+e \theta \\
\epsilon=1+\frac{3}{2} \beta \alpha, \gamma=d \alpha-c \beta, \delta=b \alpha-a \beta .
\end{gathered}
$$

The numbers $a, b, c, d$ satisfy the relation $a d-b c=1$ and are real even supernumbers. The numbers $\alpha$ and $\beta$ are odd supernumbers with property $\bar{\alpha}=i \alpha, \bar{\beta}=i \beta . A, B$ and $\Gamma$ must be multliplied by $K=1+\frac{1}{2} \alpha \beta$ to give the correct normalization $\operatorname{sdet} T=1$ (see Eq.(II.64)). I denote these quantities by $\hat{A}, \hat{B}$ and $\hat{\Gamma}$, respectively. Throughout this appendix I assume that $\gamma \in \Gamma$ is a $S P L(2 R)$ transformation (but do not confuse with $\gamma=d \alpha-c \beta$; the correct distinction between these two quantities is in general clear by contexi).

Let us introduce the number

$$
Z_{Z \overline{W^{\prime}}}:=z-\bar{u}+i \theta \bar{\nu}, \quad \Rightarrow \quad Y=\frac{1}{2 i} Z_{Z \bar{Z}}=\frac{1}{2 i}(z-\bar{z}+i \theta \bar{\theta}) .
$$

$Z_{Z U^{\prime}}$ is transformed under the action of $\gamma \in S P L(2, \mathbf{R})$ into

$$
Z_{Z \dot{W}} \mapsto Z_{Z \dot{W}}=Z_{Z \dot{W}}^{\prime}=\frac{A(Z) \tilde{B}\left(W^{\top}\right)-\bar{A}(W) B(Z)+i \Gamma(Z) \ddot{\Gamma}(W)}{B(Z) \bar{B}\left(W^{\top}\right)}
$$

Let us first consider the first two terms in the numerator of Eq.(5):

$$
A(Z) \bar{B}(W)-\bar{A}(W) B(Z)
$$

$=(a z+b-\theta \delta) \overline{(c w+d-\nu \eta)}-\overline{(a z+b-\theta \delta)}(c w+d-\nu \gamma)$

$=\underbrace{(a z-b)(c \bar{u}+d)-(a \bar{u}+b)(c z+d)}_{=:-z \bar{u}}$

$$
\begin{aligned}
& \underbrace{+i \bar{\nu}(a z+b)(d a-c \beta)-i \bar{\nu}(b a-a \beta \beta)(c z+d)}_{=i \bar{\nu}(\alpha=+i 3)} \\
& \underbrace{-\theta(c \bar{u} \div d)(b \alpha-a j)+\theta(d a-c \beta 3)(a \bar{u}+b)}_{=\theta\left(\alpha i i^{i}+\beta\right)} \\
& \underbrace{\left.+i \theta(b o-a \beta)(d a-c \beta) \bar{\nu}-(b a-a)^{3}\right) \bar{\nu} \theta(d \alpha-c \beta)}_{=2 i \alpha ; \theta \bar{\nu}} \\
& =:-\bar{u}+i \bar{u}(a=-3)+\theta(a \bar{u}+3)-2 i a 3 \theta \bar{u}
\end{aligned}
$$

For the third term:

$$
i \Gamma(Z) \bar{\Gamma}(W)=i(\alpha=-\beta-\theta)(a+\omega+\beta+\omega)
$$

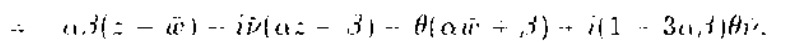

Thus combining Eqs.(6) and (7)

$$
Z_{Z \bar{W}}^{\prime}=Z_{Z \bar{W}} \frac{1}{\hat{B}(Z) \overline{\hat{B}}(W)} .
$$

Similarly

$$
z^{\prime}-w^{\prime}-\theta^{\prime} \nu^{\prime}=\frac{z-w-\theta \nu}{\hat{B}(Z) \hat{B}(W)}
$$

in particular:

$$
Y^{\prime}=Y \frac{1-\alpha \beta}{|B(Z)|^{2}}=\frac{Y}{\mid \dot{B}(Z)_{i}^{2}}
$$

Let us consider $D \theta^{\prime}$ :

$$
D \theta^{\prime}=D \frac{\Gamma(Z)}{B(Z)}=\frac{[D \Gamma(Z)] B(Z)+\Gamma(Z)[D B(Z)]}{B^{2}(Z)} .
$$

In detail:

$$
[D \Gamma(Z)] B(Z)+\Gamma(Z) ! D B(Z):=
$$$$
[\epsilon B(Z) \div \theta \alpha B(Z)+\gamma \Gamma+\Gamma \theta c](c z+d)\left(1-\frac{\alpha \beta}{2}-\frac{\theta \gamma}{c z+d}\right)
$$

and therefore

$$
\frac{[D \Gamma(Z) j B(Z)+\Gamma(Z)[D B(Z)]}{B(Z)}=1-\frac{\alpha \beta}{2}
$$

Thus

$$
D \theta^{\prime}=\frac{1-\frac{\alpha \beta}{2}}{B(Z)}=\frac{1}{\hat{B}(Z)} \equiv F_{\gamma}(Z) .
$$

With Eq.(II.52) we have the transformation properties of the operator $D$

$$
D=\frac{1-\frac{\alpha \beta}{2}}{B(Z)} D^{\prime}=\frac{1}{\hat{B}(Z)} D^{\prime}
$$

Let us consider now the $J$ acobian $J$ in the volume element for the transformation of Eq. (I): $d_{z} d \bar{\Sigma} d \theta d \bar{\theta}=J d z^{\prime} d \bar{\Sigma}^{\prime} d \theta^{\prime} d \bar{\theta}^{\prime}$. It is given by:

$$
\begin{aligned}
& J=\operatorname{sdet}\left(\begin{array}{llll}
\left(\partial_{z} z^{\prime}\right) & \left(\partial_{\bar{z}} \bar{z}^{\prime}\right) & \left(\partial_{\bar{z}} \theta^{\prime}\right) & \left(\partial_{\bar{z}} \bar{\theta}^{\prime}\right) \\
\left(\partial_{\bar{z}} \bar{z}^{\prime}\right) & \left(\partial_{\bar{z}} \bar{z}^{\prime}\right) & \left(\partial_{\bar{z}} \theta^{\prime}\right) & \left(\partial_{\bar{z}} \bar{\theta}^{\prime}\right) \\
\left(\partial_{\theta} z^{\prime}\right) & \left(\partial_{\theta} \bar{z}^{\prime}\right) & \left(\partial_{\theta} \theta^{\prime}\right) & \left(\partial_{\theta} \bar{\theta}^{\prime}\right) \\
\left(\partial_{\bar{\theta}} \bar{z}^{\prime}\right) & \left(\partial_{\bar{\theta}} \bar{z}^{\prime}\right) & \left(\partial_{\bar{\theta}} \theta^{\prime}\right) & \left(\partial_{\dot{\theta}} \bar{\theta}^{\prime}\right)
\end{array}\right) \\
& =\operatorname{sdet}\left(\begin{array}{cccc}
\left(\partial_{z} z^{\prime}\right) & 0 & \left(\partial_{z} \theta^{\prime}\right) & 0 \\
0 & \left(\partial_{\bar{\Sigma}} \bar{\Sigma}^{\prime}\right) & 0 & \left(\partial_{\bar{z}} \bar{\theta}^{\prime}\right) \\
\left(\partial_{\theta} z^{\prime}\right) & 0 & \left(\partial_{\theta} \theta^{\prime}\right) & 0 \\
0 & \left(\partial_{\bar{\theta}} \bar{z}^{\prime}\right) & 0 & \left(\partial_{\bar{\theta}} \bar{\theta}^{\prime}\right)
\end{array}\right) \\
& -\frac{1}{\left(\partial_{\theta} \theta^{\prime}\right)\left(\partial_{\theta^{\prime}} \bar{\theta}^{\prime}\right)} \operatorname{det}\left[\left(\begin{array}{cc}
\left(\partial_{\Sigma} z^{\prime}\right) & 0 \\
0 & \left(\partial_{\Sigma} \bar{z}^{\prime}\right)
\end{array}\right)\right. \\
& \left.-\left(\begin{array}{cc}
\left(\partial_{\dot{*}} \theta^{\prime}\right) & 0 \\
0 & \left(\partial_{\bar{\Sigma}} \bar{\theta}^{\prime}\right)
\end{array}\right) \cdot\left(\begin{array}{cc}
\left(\partial_{\theta} \theta^{\prime}\right)^{-1} & 0 \\
0 & \left(\partial_{\bar{\theta}} \dot{\theta}^{\prime}\right)^{-1}
\end{array}\right) \cdot\left(\begin{array}{cc}
\left(\partial_{\theta} z^{\prime}\right) & 0 \\
0 & \left(\partial_{\bar{\theta}} \bar{z}^{\prime}\right)
\end{array}\right)\right]
\end{aligned}
$$

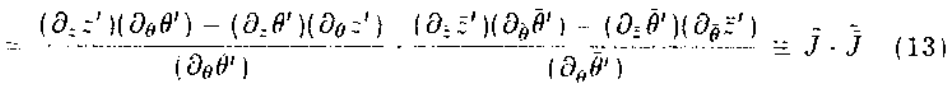


and we see that the Jacobian factorizes. Let us calculate $\bar{J}$. It is given by $\tilde{J}=\frac{\left[\left(\partial_{z} A\right) B-A\left(\partial_{2} B\right)\right]\left[\left(\partial_{\theta} \Gamma\right) B+\Gamma\left(\partial_{\theta} B\right)\right]-\left[\left(\partial_{z} \Gamma\right) B-\Gamma\left(\partial_{z} B\right) !\left(\partial_{\theta} A\right) B-A\left(\partial_{\theta} B\right)\right.}{\left[\left(\partial_{\theta} \Gamma\right) B+\Gamma\left(\partial_{\theta} B\right)\right]^{2}}$.

For the denominator:

$$
\left\{\left(\partial_{\theta} \Gamma\right) B+\Gamma\left(\partial_{\theta} B\right)\right]^{2}=(\epsilon B-\Gamma \gamma)^{2}=B(c z+d)\left[1-\alpha \beta-3 \theta \frac{d \alpha-c \beta}{c z+d}\right]
$$

and therefore

$$
\frac{1}{\left[\left(\partial_{\theta} \Gamma\right) B+\Gamma\left(\partial_{\theta} B\right)_{i}^{i 2}\right.}=\frac{1}{B(c z+d)}\left[1+\alpha \beta+3 \theta \frac{d \alpha-c \beta}{c z+d}\right] .
$$

For the numerator:

$\left\{\left(\partial_{z} A\right) B-A\left(\partial_{z} B\right)\right]\left[\left(\partial_{\theta} \Gamma\right) B+\Gamma\left(\partial_{\theta} B\right)\right\}-\left[\left(\partial_{z} \Gamma\right) B-\Gamma\left(\partial_{z} B\right)\right\}\left[\left(\partial_{\theta} A\right) B-A\left(\partial_{\theta} B\right)\right]$

$=(a B-A c)(\epsilon B-\Gamma \gamma)-(\alpha B-c \Gamma)\left(-A_{\gamma}-\delta B\right)$

$=\underbrace{e\left(a B^{2}-A B c-a b \theta \gamma+2 A c \theta \gamma+c \theta \delta B\right)}$

$(c z+d)\left[1-\frac{3}{2} \alpha \beta-3 \theta^{\frac{d \alpha}{c}-c \beta}+\frac{c \beta}{+d}\right]$

$+\underbrace{(\alpha z+d)(2 A c \gamma-\alpha B \gamma-c \delta B)}_{=\alpha \beta(\mathrm{c} z+d)}+\underbrace{\alpha \delta B^{2}-\alpha \gamma A B}_{=-\alpha \beta(c z+d)}$

$$
=\left(c_{z}+d\right)\left[1-\frac{3}{2} \alpha \beta-3 \theta \frac{d \alpha-c \beta}{c z+d}\right] \text {. }
$$

Thus:

$$
j=\frac{1-\frac{1}{2} \alpha \beta}{B(Z)}=\frac{1}{\hat{B}(Z)}, \quad \Rightarrow \quad J=\frac{1-\alpha \beta}{|B(Z)|^{2}}=\frac{1}{\hat{i}\left(\left.\bar{B}(Z)\right|^{2}\right.} .
$$

Combining Eqs.(10) and (18), the invariance of $d V^{r}(Z)$ is proven, i.e.

$$
\frac{d z d \bar{z} d \theta d \dot{\theta}}{2 Y}=\frac{d z^{\prime} d \bar{z}^{\prime} d \theta^{\prime} d \bar{\theta}^{\prime}}{2 Y^{\prime}}
$$

\section{The Invariance of $R(Z, W)$}

Let us consider the two-point quantities $r(Z, W)$ and $R(Z, W)$. They are given

$$
\begin{aligned}
R(Z, W)= & \frac{\mid z-w-\theta \nu^{\prime 2}}{Y V}=\frac{|z-w-\theta \nu|^{2}}{(y+\theta \bar{\theta} / 2)(v+\nu \bar{\nu} / 2)} \\
r(Z, W)= & \frac{2 \theta \bar{\theta}+i(\nu-i \bar{\nu})(\theta+i \bar{\theta})}{4 Y}-\frac{2 \nu \bar{\nu}+i(\theta-i \bar{\theta})(\nu+i \bar{\nu})}{4 V} \\
& \quad+\frac{(\nu+i \bar{\nu})(\theta+i \bar{\theta}) \operatorname{Re}(z-w-\theta \nu)}{4 Y Y} .-w
\end{aligned}
$$

Furthermore we have $\cosh d(Z, W)=1+\frac{1}{2} R(Z, W)-2 r(Z, W)$. Of course, the invariance of $d$ follows from the invariance of $r$ and $R$.

Let us consider $R$. From Eqs. (8) and (9) we see at once that

$$
R(\gamma Z, \gamma W)=R(Z, W)
$$

and the invariance of $R$ with respect to the action of $S P L(2 \mathbf{R})$ is shown. The invariance of $r(Z, W)$ is similar but nuch more involved and will not be given here.

\section{The Vierbein $E^{A}$}

Let us consider the quantity $E^{2}=(d z+\theta d \theta) / Y$ under the action of the transformation (1). First with $E_{\text {q. }}(10)$

$$
Y^{\prime}=\gamma Y=\frac{Y}{|\hat{B}(Z)|^{2}} .
$$

Next this gives

$$
\begin{aligned}
& d z^{\prime}+\theta^{\prime} d \theta^{\prime}=\gamma(d z+\theta d \theta) \\
& =\frac{1}{B}\left(\frac{\partial A}{\partial z} d z-\frac{\partial A}{\partial \theta} d \theta\right)-\frac{1}{B^{2}}\left(\frac{\partial B}{\partial z} d z-\frac{\partial B}{\partial \theta} d \theta\right) \\
& +\frac{\Gamma}{B}\left[\frac{1}{B}\left(\frac{\partial \Gamma}{\partial z} d z-\frac{\partial \Gamma}{\partial \theta} d \theta\right)-\frac{\Gamma}{B^{2}}\left(\frac{\partial B}{\partial z} d z-\frac{\partial B}{\partial \theta} d \theta\right)\right] \\
& =\frac{1}{B^{2}}\left[\left(B a-A_{C}+\Gamma_{0}\right) d z-\left(B \delta-A_{\gamma}-\Gamma_{\epsilon}\right) d \theta_{j}=\frac{d z \div \theta d \theta}{\dot{B}^{2}(Z)}\right. \text {. }
\end{aligned}
$$

Thus combining Eqs.(23) and (24) we get

$$
E^{\prime z}=\gamma E^{z}=\epsilon^{-i \phi} E^{z}
$$

with some phase factor given by $\phi=\operatorname{Im}(B) / \operatorname{Re}(B)$. Therefore $E^{z}$ (and $E^{\bar{z}}$ ) are up to phase factors invariant under the action of $S P L(2, \mathbf{R})$. For the combination $E^{z} E^{z}$ this phase factor drops out. The calculation for $E^{\theta}$ (and $E^{\bar{\theta}}$ ) is similar.

\section{Appendix B: Proof of the Path Integral on $S H$}

In this Appendix I want to show that from the short time kernel of the path integral on $S \mathcal{H}$ ! I denote $Z=(z, \theta), Y_{M}=y_{M}+\theta_{M} \bar{\theta}_{M} / 2$. where $y_{M}=\left(y^{\prime}+y^{\prime \prime}\right) / 2$ $\theta_{M}=\left(\theta^{\prime}+\theta^{\prime \prime}\right) / 2, \Delta z=\left(z^{\prime \prime}-z^{\prime}\right), \Delta \theta=\left(\theta^{\prime \prime}-\theta^{\prime}\right)$ and h.c. $)$

$$
\begin{aligned}
& K\left(Z^{\prime} . Z^{\prime \prime} ; \epsilon\right)=\frac{\sqrt{\left.Y^{\prime}\right)^{\prime \prime}}}{2 \pi i Y_{M}} \\
& \quad \times \exp \left\{\frac{i m}{2 \epsilon Y_{M}^{2}}\left[\Delta z \Delta z-i \bar{\theta}_{M} \Delta z \Delta \theta-i \theta_{M} \Delta z \Delta \ddot{\theta}-\left(2 Y_{M}+\theta_{M} \bar{\theta}_{M}\right) \Delta \theta \Delta \bar{\theta}\right]\right\} \quad(1)
\end{aligned}
$$

and the time evolution equation

$$
\Psi(Z ; t+\epsilon)=\int \frac{d x^{\prime} d y^{\prime} d \theta^{\prime} d \bar{\theta}^{\prime}}{2 Y^{\prime}} K^{\prime}\left(Z, Z^{\prime} ; \epsilon\right) \Psi\left(Z^{\prime}: t\right)
$$

the "super"-Schrödinger equation can be derived, i.e. $\left(=:=x+i y . \theta=\theta_{1}+i \theta_{2}\right)$ :

$$
\begin{aligned}
& i \frac{\partial \Psi(Z ; t)}{\partial t}--\frac{Y}{m}\left[(2 Y-\theta \bar{\theta}) \partial_{z} \partial_{\bar{\xi}}+i \bar{\theta} \partial_{z} \partial_{\bar{\theta}}-i \theta \partial_{\bar{z}} \partial_{\theta}+\partial_{\bar{\theta}} \partial_{\theta}\right] \Psi(Z, t) \\
& =-\frac{1}{2 m}\left[y\left(y+i \theta_{2} \theta_{3}\right)\left(\partial_{x}^{2}+\partial_{y}^{2}\right)+i\left(y+i \theta_{2} \theta_{1}\right) \partial_{\theta_{2}} \partial_{\theta_{1}}\right. \\
& \left.+y\left(\theta_{1} \partial_{y} \partial_{\theta_{1}}+\theta_{2} \partial_{y} \partial_{\theta_{2}}+\theta_{2} \partial_{x} \partial_{\theta_{1}}-\theta_{1} \partial_{x} \partial_{\theta_{z}}\right)\right] \Psi(Z, t)
\end{aligned}
$$


One has to perform a Taylor expansion in Eq. (2):

$$
\Psi(Z ; t)+\epsilon \frac{\partial}{\partial t} \Psi(z ; t)=B_{0} \Psi(z ; t)+B_{a} \partial_{a} \Psi(Z ; t)+B_{a b} \partial_{b} \partial_{a} \Psi(Z ; t)+\cdots,
$$

where the coefficients $B_{a}$ and $B_{a b}$ in terms of the real coordinates $x, y, \theta_{1}, \theta_{2}$ up to $O(\epsilon)$ are given by

$$
\begin{aligned}
& B_{0}=\int \frac{d x^{\prime} d y^{\prime} d \theta_{1}^{\prime} d \theta_{2}^{\prime}}{2\left(y^{\prime}+i \theta_{2}^{\prime} \theta_{1}^{\prime}\right)} K\left(Z, Z^{\prime} ; \epsilon\right) \\
&=\frac{\sqrt{y+i \theta_{2} \theta_{1}}}{4 \pi i} \int \frac{d x^{\prime} d y^{\prime} d \theta_{1}^{\prime} d \theta_{2}^{\prime}}{\sqrt{y^{\prime}+i \theta_{2}^{\prime} \bar{\theta}_{1}^{\prime}\left(y_{M}+i \theta_{M 2} \theta_{M 1}\right)}} \\
& \times \exp \left\{\frac { i m } { 2 \epsilon Y _ { M } ^ { 2 } } \left[\Delta z \Delta \bar{z}-i \bar{\theta}_{M} \Delta \bar{z} \Delta \theta-i \theta_{M} \Delta z \Delta \bar{\theta}-\left(2 Y_{M}+\theta_{M}\right.\right.\right. \\
& B_{x}=\int \frac{d x^{\prime} d y^{\prime} d \theta_{1}^{\prime} d \theta_{2}^{\prime}}{2\left(y^{\prime}+i \theta_{2}^{\prime} \theta_{1}^{\prime}\right)} K\left(Z, Z^{\prime} ; \epsilon\right)\left(x-x^{\prime}\right) \simeq 0 \\
& B_{y}=\int \frac{d x^{\prime} d y^{\prime} d \theta_{1}^{\prime} d \theta_{2}^{\prime}}{2\left(y^{\prime}+i \theta_{2}^{\prime} \theta_{1}^{\prime}\right)} K\left(Z, Z^{\prime} ; \epsilon\right)\left(y-y^{\prime}\right) \simeq 0 \\
& B_{\theta_{1}}=\int \frac{d x^{\prime} d y^{\prime} d \theta_{1}^{\prime} d \theta_{2}^{\prime}}{2\left(y^{\prime}+i \theta_{2}^{\prime} \theta_{1}^{\prime}\right)} K\left(Z, Z^{\prime} ; \epsilon\right)\left(\theta_{1}-\theta_{1}^{\prime}\right) \simeq 0 \\
& B_{\theta_{2}}=\int \frac{d x^{\prime} d y^{\prime} d \theta_{1}^{\prime} d \theta_{2}^{\prime}}{2\left(y^{\prime}+i \theta_{2}^{\prime} \theta_{1}^{\prime}\right)} K\left(Z, Z^{\prime} ; \epsilon\right)\left(\theta_{2}-\theta_{2}^{\prime}\right) \simeq 0 .
\end{aligned}
$$$$
\times \exp \left\{\frac{i m}{2 \epsilon Y_{M}^{2}}\left[\Delta z \Delta \bar{z}-i \bar{\theta}_{M} \Delta \bar{z} \Delta \theta-i \theta_{M} \Delta z \Delta \bar{\theta}-\left(2 Y_{M}+\theta_{M} \bar{\theta}_{M}\right) \Delta \theta \Delta \bar{\theta}\right]\right\} \simeq 1 .
$$

$B_{x x}=\frac{1}{2} \int \frac{d x^{\prime} d y^{\prime} d \theta_{1}^{\prime} d \theta_{2}^{\prime}}{2\left(y^{\prime}+i \theta_{2}^{\prime} \theta_{1}^{\prime}\right)} K^{\prime}\left(Z, Z^{\prime} ; \epsilon\right)\left(x-x^{\prime}\right)^{2} \simeq \frac{i \epsilon}{2 m} y\left(y+i \theta_{2} \theta_{1}\right)$

$B_{y y}=\frac{1}{2} \int \frac{d x^{\prime} d y^{\prime} d \theta_{1}^{\prime} d \theta_{2}^{\prime}}{2\left(y^{\prime}+i \theta_{2}^{\prime} \theta_{1}^{\prime}\right)} K\left(Z ; Z^{\prime} ; \epsilon\right)\left(y-y^{\prime}\right)^{2} \simeq \frac{i \epsilon}{2 m} y\left(y+i \theta_{2} \theta_{1}\right)$

$B_{\theta_{1} \theta_{2}}=\frac{1}{2} \int \frac{d x^{\prime} d y^{\prime} d \theta_{1}^{\prime} d \theta_{2}^{\prime}}{2\left(y^{\prime}+i \theta_{2}^{\prime} \theta_{1}^{\prime}\right)} K\left(Z, Z^{\prime} ; \epsilon\right)\left(\theta_{1}-\theta_{1}^{\prime}\right)\left(\theta_{2}-\theta_{2}^{\prime}\right) \simeq \frac{i \epsilon}{2 m} i\left(y+i \theta_{2} \theta_{1}\right)$

$B_{x \theta_{1}}=\frac{1}{2} \int \frac{d x^{\prime} d y^{\prime} d \theta_{1}^{\prime} d \theta_{2}^{\prime}}{2\left(y^{\prime}+i \theta_{2}^{\prime} \theta_{1}^{\prime}\right)} K\left(Z, Z^{\prime}: \epsilon\right)\left(x-x^{\prime}\right)\left(\theta_{3}-\theta_{1}^{\prime}\right) \simeq \frac{i \epsilon}{4 m} y \theta_{2}$

$B_{x \theta_{2}}=\frac{1}{2} \int \frac{d x^{\prime} d y^{\prime} d \theta_{1}^{\prime} d \theta_{2}^{\prime}}{2\left(y^{\prime}+i \theta_{2}^{\prime} \theta_{1}^{\prime}\right)} K\left(Z, Z^{\prime} ; \epsilon\right)\left(x-x^{\prime}\right)\left(\theta_{2}-\theta_{2}^{\prime}\right) \simeq \frac{i \epsilon}{4 m}\left(-y \theta_{1}\right)$

$B_{y \theta_{1}}=\frac{1}{2} \int \frac{d x^{\prime} d y^{\prime} d \theta_{1}^{\prime} d \theta_{2}^{\prime}}{2\left(y^{\prime}+i \theta_{2}^{\prime} \theta_{1}^{\prime}\right)} K\left(Z, Z^{\prime} ; \epsilon\right)\left(y-y^{\prime}\right)\left(\theta_{1}-\theta_{1}^{\prime}\right) \simeq \frac{i \epsilon}{4 m} y \theta_{1}$

$B_{y \theta_{2}}=\frac{1}{2} \int \frac{d x^{\prime} d y^{\prime} d \theta_{1}^{\prime} d \theta_{2}^{\prime}}{2\left(y^{\prime}+i \theta_{2}^{\prime} \theta_{1}^{\prime}\right)} K^{-1}\left(Z \cdot Z^{\prime}: \epsilon\right)\left(y-y^{\prime}\right)\left(\theta_{2}-\theta_{2}^{\prime}\right) \simeq \frac{i \epsilon}{4 m} y \theta_{2}$

$B_{x y}=\frac{1}{2} \int \frac{d x^{\prime} d y^{\prime} d \theta_{1}^{\prime} d \theta_{2}^{\prime}}{2\left(y^{\prime}+i \theta_{2}^{\prime} \theta_{1}^{\prime}\right)} h\left(Z, Z^{\prime} ; \epsilon\right)\left(x-x^{\prime}\right)\left(y-y^{\prime}\right) \simeq 0$.

I just present some details of the calculation of $B_{0}$ : the other coefficients are calculated in a similar way.' First let us consider the Lagrangian in the midpoint formulation on the lattice:

$$
\left.L=\frac{m}{2 \epsilon^{2} Y_{M}^{2}} \Delta=\Delta z-i \bar{\theta}_{M} \Delta \bar{z} \Delta \theta-i \theta_{M} \Delta=\Delta \bar{\theta}-\left(2 Y_{M}=\theta_{M} \bar{\theta}_{M}\right) \Delta \theta \Delta \bar{\theta}\right\} .
$$

The exponential expanded in the Grassmann variables yields $\left[\Delta x=x-x^{\prime}, \Delta y=\right.$ $\left.y-y^{\prime}\right]$

$$
\begin{aligned}
\epsilon^{i \epsilon L} & =\exp \left[-\frac{m}{2 i \epsilon} \frac{\left(x-x^{\prime}\right)^{2}+\left(y-y^{\prime}\right)^{2}}{y_{M}^{2}}\right] \\
\times\{1 & -\frac{m^{2}}{4 \epsilon^{2} y_{M}^{4}} \bar{\theta} \theta \bar{\theta}^{\prime} \theta^{\prime}\left(\Delta^{2} x+\Delta^{2} y\right) \\
& +\frac{i m}{\epsilon y_{M}} \theta \bar{\theta}^{\prime}\left[1+\frac{i \Delta x}{2 y_{M}}-\frac{\Delta^{2} x+\Delta^{2} y}{8 y_{M}^{2}}\right]+\frac{i m}{\epsilon y_{M}} \theta^{\prime} \bar{\theta}\left[1-\frac{i \Delta x}{2 y_{M}}-\frac{\Delta^{2} x+\Delta^{2} y}{8 y_{M}^{2}}\right] \\
& \left.+\frac{i m}{\epsilon y_{M}} \theta \bar{\theta}\left[1-\frac{\Delta y}{2 y_{M}}+\frac{\Delta^{2} x+\Delta^{2} y}{8 y_{M}^{2}}\right]+\frac{i m}{\epsilon y_{M}} \theta^{\prime} \bar{\theta}^{\prime}\left[1+\frac{\Delta y}{2 y_{M}}+\frac{\Delta^{2} x+\Delta^{2} y}{8 y_{M}^{2}}\right]\right\}
\end{aligned}
$$

Furthermore we have

$$
\begin{aligned}
\frac{1}{Y_{M}} & =\frac{1}{y_{M}}\left[1+\frac{\bar{\theta} \theta^{\prime}-\theta^{\prime} \bar{\theta}^{\prime}-\theta \bar{\theta}^{\prime}-\theta \bar{\theta}}{8 y_{M}}\right] \\
\sqrt{\frac{Y^{\prime \prime}}{Y^{\prime}}} & =\sqrt{\frac{y^{\prime \prime}}{y^{\prime}}}\left[1+\frac{\bar{\theta}^{\prime} \theta^{\prime}}{4 y^{\prime}}-\frac{\bar{\theta} \theta}{4 y}-\frac{\bar{\theta} \theta \bar{\theta}^{\prime} \theta^{\prime}}{16 y y^{\prime}}\right] .
\end{aligned}
$$

In the usual way $y^{\prime}$ and $y_{M}$ have to be expanded according to

$$
\frac{1}{y_{M}} \simeq \frac{1}{y}\left[1+\frac{\Delta y}{2 y}+\frac{\Delta^{2} y}{4 y^{2}}\right], \quad \frac{1}{\sqrt{y}} \simeq \frac{1}{\sqrt{y}}\left[1+\frac{\Delta y}{2 y}+\frac{3}{8} \frac{\Delta^{2} y}{y^{2}}\right] .
$$

In multiplication the various terms and inserting into the Taylor expansions we have to take into account terms up to the sixth and eighth order in $\Delta x$ and $\Delta y$, respectively. Explicitly, after integrating out the Grassmann variables

$$
\begin{aligned}
& B_{0} \simeq \frac{m}{2 \pi i \epsilon y^{2}} \underbrace{\iint d x^{\prime} d y^{\prime} \exp \left[-\frac{m}{2 i \epsilon} \frac{\Delta^{2} x+\Delta^{2} y}{y^{2}}\right]}_{\simeq \frac{2 \pi i \epsilon}{m} y^{2}(\epsilon-0)} \\
& \times\left\{1+\frac{3 \pi i \epsilon}{8 m}+\frac{\Delta^{2} x+22 \Delta^{2} y}{8 y^{2}}\right. \\
& +\frac{11 i m}{8 \epsilon y^{4}}\left(\Delta^{2} x \Delta^{2} y+\Delta^{4} y\right)-\frac{m^{2}}{8 \epsilon^{2} y^{6}}\left(\Delta^{4} x \Delta^{2} y+2 \Delta^{2} x \Delta^{4} y+\Delta^{4} y\right)
\end{aligned}
$$

One makes use of the following identities

$$
\begin{array}{lrl}
\Delta^{2} x & =\Delta^{2} y \doteq \frac{i \epsilon}{m} y^{2} . & \Delta^{6} x=\Delta^{6} y \doteq 15\left(\frac{i \epsilon}{m} y^{2}\right)^{3} \\
\Delta^{4} x=\Delta^{4} y \doteq 3\left(\frac{i \epsilon}{m} y^{2}\right)^{2}, & \Delta^{8} y \doteq 105\left(\frac{i \epsilon}{m} y^{2}\right)^{4}
\end{array}
$$

respecting that in the required limit the integral over $x$ and $y$ gives $2 \pi i \epsilon y^{2} / m$ (as denoted , the result of Eq.(5) is proven.

The calculatious were rlome with the help of the algebraic computer program REllt:('F: 


\section{Appendix C: The Heat Kernel on SH}

In this Appendix I want to give a short summary of the results of Aoki [3] who first calculated the heat kernel on $S \mathcal{H}$. Let us first describe his definition of the Laplacian he uses. It reads

$$
\Delta_{m}:=\Delta_{0}+m(z-\bar{z}-\theta \bar{\theta})\left[\hat{D} \hat{\bar{D}}+\frac{1}{2}\left(\partial_{z}-\partial_{\bar{z}}\right),-\frac{m^{2}}{2}(\theta-\bar{\theta})(\hat{D}+\overline{\bar{D}})+\frac{m^{2}}{4},\right.
$$

where $m \in \mathbf{Z}, \hat{D}=\partial_{\theta}+\theta \partial_{z}, \hat{\bar{D}}=\partial_{\dot{\theta}}+\bar{\theta} \partial_{\Sigma}$ and

$\Delta_{0}=\left[(z-\bar{z}-\theta \bar{\theta})^{2}+\theta \bar{\theta}(z-\bar{z}-\theta \bar{\theta})\right] \partial_{\Sigma} \partial_{\bar{\Sigma}}+(z-\bar{z}-\theta \bar{\theta})\left(\partial_{\theta} \partial_{\bar{\theta}}+\theta \partial_{\theta} \partial_{\bar{z}}-\bar{\theta} \partial_{\bar{\theta}} \partial_{\bar{z}}\right) .(2)$

Now recall the Laplacians $\square_{m}$ and $\widehat{\square}_{m}$ used by Baranov et al. $\mid 10$. Thus we find the following equivalence

$$
\left(\hat{\square}_{m}+\frac{m}{2}\right)^{2}=\Delta_{m},
$$

where

$$
\begin{aligned}
& \hat{\square}_{m}=(z-\bar{z}-\theta \bar{\theta}) \hat{D} \hat{\bar{D}}-\frac{m}{2}(\theta-\bar{\theta})(\hat{D}+\dot{\bar{D}}) \\
& \square_{m}=(z-\bar{z}-\theta \bar{\theta})^{\frac{m}{2}}\left(\hat{\square}_{m}+\frac{m}{2}\right)(z-\bar{z}-\theta \bar{\theta})^{-\frac{m}{2}} .
\end{aligned}
$$

These equations establish the unitary equivalences of $\Delta_{m}$ and $\square_{m}^{2}$, i.e. $\Delta_{m} \cong \square_{m}^{2}$ This gives, of course, also $\square_{m} \cong \widehat{\square}_{m}+\frac{m}{2}$ in my notation.

Aoki studies the heat kernel $\hat{G}_{M}^{t}$ which is defined by $[Z=(z, \theta), W=(w, \nu)=$ $(u+i v, \nu)]$ :

$$
\begin{aligned}
\left(\partial_{t}+\Delta_{m}\right) \hat{G}_{m}^{t}(Z, W) & =0 \quad(t>0) \\
\hat{G}_{m}^{t}(Z, W) & \rightarrow \frac{z-\bar{z}-\theta \bar{\theta}}{-4} \delta(x-u) \delta(y-v)(\theta-\nu)(\bar{\theta}-\bar{\nu}) \quad\left(t \rightarrow 0^{+}\right)
\end{aligned}
$$

Now consider some redefinitions, i.e. define $G_{m}^{t}$ by

$$
\hat{G}_{\mathrm{m}}^{t}(Z, W)=\left(\frac{z-\bar{u}-\theta \bar{\nu}}{w-\bar{z}-\bar{\theta} \nu}\right)^{\frac{m}{2}} G_{m}^{t}(Z, W)
$$

then

$$
\begin{aligned}
\left(\partial_{t}+\bar{\Delta}\right) G_{m}^{t}(Z, W) & =0 \quad(t>0) \\
\frac{1}{\sinh d(\bar{Z}, W)} G_{m}^{t}\left(Z, W^{+}\right) & \rightarrow-\frac{1}{2 \pi} \delta[\cosh d(Z, W)-1] i \Delta(Z, W) \bar{\Delta}(Z, W) \quad\left(t \rightarrow 0^{+}\right) .
\end{aligned}
$$

Here $\tilde{\Delta}_{m}$ is a transformed Laplacian and $d, \Delta$ and $\bar{\Delta}$ are $S P L(2, \mathbf{R})$ invariant quantities given by:

$$
\begin{aligned}
\bar{\Delta}_{m} & =\left(\frac{z_{1 \overline{2}}}{z_{2 \overline{1}}}\right)^{-\frac{m}{2}} \Delta_{m}\left(\frac{z_{1 \overline{2}} \overline{2}}{z_{2 \overline{1}}}\right)^{\frac{m}{2}} \\
\cosh d(Z, W) & =1-2 \frac{z_{12} z_{\overline{1} \overline{2}}}{z_{1 \overline{\mathrm{j}}} z_{2 \overline{2}}} \\
\Delta(Z, W) & =\frac{\theta z_{1 \bar{L}}+\nu z_{\overline{2} 1}+\bar{\nu} z_{12}+\theta \nu \bar{\nu}}{\sqrt{z_{12} z_{2 \overline{2}} z_{\overline{2} 1}}} \\
\tilde{z}_{a b} & =z_{a}-z_{b}-\theta_{a} \theta_{b}, \quad(a, b=1,2), \quad Z_{1}=Z, Z_{2}=W .
\end{aligned}
$$

$$
G_{m}^{t}\left(Z, W^{-}\right)=g_{m}^{t}(r)+i \Delta \Delta h_{n}^{t}(d) .
$$

This leads to coupled differential equations for $g_{m}^{t}$ and $h_{m}^{t}$ :

$$
\begin{aligned}
& \left\{\frac{\partial}{\partial t}-\left[\frac{\partial^{2}}{\partial d^{2}}+\left(\frac{1}{\sinh d}-m \tanh \frac{d}{2}\right) \frac{\partial}{\partial d}+\frac{m^{2}}{4}\left(1+\frac{2}{\cosh d+1}\right)\right]\right\} g_{m}^{t}(d) \\
& =\frac{2(m+1)}{\sinh d} h_{m}^{t}(d) \\
& \left\{\frac{\partial}{\partial t}-\left[\frac{\partial^{2}}{\partial d^{2}} \cdots\left(-\frac{1}{\sinh d}-m \tanh \frac{d}{2}\right) \frac{\partial}{\partial d}+\frac{\cosh d}{\sinh ^{2} d}-\frac{m\left(1+\frac{m}{2}\right)}{\cosh r+1}+\frac{m^{2}}{4}\right]\right\} h_{m}^{t}(d) \\
& -m \frac{\sinh d}{\cosh d+1}\left(\frac{\partial^{2}}{\partial d^{2}}+\frac{1}{\sinh d} \frac{\partial}{\partial d}-\frac{m / 2}{\cosh d}-\frac{m^{2}}{4}\right) g_{m}^{t}(d)=0
\end{aligned}
$$

with the boundary conditions:

$$
\begin{aligned}
g_{m}^{t}(d) & \rightarrow 0 \quad\left(t \rightarrow 0^{+}\right) \\
\frac{1}{\sinh }-h_{m}^{t}(d) & \rightarrow-\frac{1}{2 \pi} \delta(\cosh d-1) \quad\left(t \rightarrow 0^{t}\right) .
\end{aligned}
$$

The solutions for $g_{m}^{t}$ and $h_{m}^{t}$ read

$$
\begin{aligned}
& g_{m}^{t}(d)=-\frac{1}{2 \pi^{\frac{3}{2}+\frac{1}{2}}} \int_{d}^{\infty} \frac{\sinh \frac{m+1}{2} u \epsilon^{-u^{2} / 4 t}}{\sqrt{2(\cosh u-\cosh d)}} T_{m}\left(\frac{\cosh \frac{u}{2}}{\cosh \frac{d}{2}}\right) d u \\
& h_{m}^{t}(d)=-\frac{\sinh d}{4 \pi^{\frac{3}{2}} \frac{3}{2}} \int_{d}^{\infty} \frac{\cosh \left(\frac{m}{2} u\right) \epsilon^{-u^{2} / 4 t}}{\sqrt{2(\cosh u-\cdots \cosh d)}} T_{m+1}\left(\frac{\cosh \frac{u}{2}}{\cosh \frac{d}{2}}\right) d u-\frac{m}{2} \tanh \frac{d}{2} g_{m}^{t}(d)
\end{aligned}
$$

where $T_{k}\left(k \in \mathbf{N}_{0}\right)$ are Chebyshev-polynomials. One can also expand $\hat{G}_{m}^{\prime}$ as

$$
\hat{G}_{m}^{t}(Z, W)=\hat{g}_{M}^{t}(z, w)+\frac{\theta \bar{\theta}}{z-\bar{z}} \hat{F}_{m}^{t}(z, w)+\text { terms involving } \nu \bar{\nu}
$$

with the relation

$$
\left[g_{m}^{t}(d)\right]_{\text {Body }}-\left(\frac{z-\bar{w}}{w^{\prime}-\bar{z}}\right)^{-\frac{m}{2}} \hat{g}_{m}^{t}\left(z, w^{\prime}\right) .
$$

As is shown on Ref.[3] the following Laplace representation can be deduced for $\hat{g}_{m}^{t}$ :

$$
\hat{g}_{m}^{t}(z, u)=-(m+1) \int_{c+i R} \frac{d s}{2 \pi i} \int_{-\infty}^{\infty} d k \int_{0}^{\infty} d p \frac{\epsilon^{s t} \Psi_{p, k}(x, y) \bar{\Psi}_{p, k}(u, v)}{\left(\frac{m i 1}{2}-i p\right)^{2} j\left[s-\left(\frac{m+1}{2}-i p\right)^{2} !\right.} \text {. }
$$

where the functions $\Psi_{p, k}$ are the normalized Eigenfunctions of the Maass operator $D_{-n}=y^{2}\left(\partial_{x}^{2}+\partial_{y}^{2}\right)+2 i m \partial_{x}$ and are given by $[3,18,39]\left(\mu \equiv-\operatorname{sign}(k) \frac{m}{2}, p>0, k \in \mathbf{R}\right)$;

$$
\Psi_{p, k}(x, y)=\left|\Gamma\left(\frac{1}{2}-\mu+i p\right)\right| \sqrt{\frac{p \sinh 2 \pi p}{4 \pi^{3}|k|}} \epsilon^{i k x} \zeta_{\mu, i p}(2|k| y),
$$

where the $W_{\mu, \nu}(z)$ are Whittacker functions. The representation (15) shows a cut in the complex $s$-plane located along the critical line Res $=\frac{m+1}{2}$ which gives the spectrum of $\sqrt{\Delta_{m}} \cong \hat{\square}_{m}+\frac{m}{2} \cong \square_{m}$ reading $\lambda=\frac{m+1}{2} \pm i p(p>0)$.

(1)

1)


appendix D: The Path Integral on the Poincaré Upper Half-Plane $\mathcal{H}$

In this Appendix I present three diferent path integral treatments for a particle moving freely on the Poincaré upper half-plane $\mathcal{H} \equiv\{z=x+i y \mid y>0\}$, endowed with the hyperbolic geometry. The Poincaré upper half-plane is analytically equivalent to three further Riemannian spaces; the pseudosphere $\Lambda^{2}$, the Poincare disc $D$ and the hyperbolic strip $S$ (as already noted in the introduction).

For a review of classical and quantum mechanical motion (in bounded and unbounded domains) in these four Riemannian spaces, see e.g. Balazs/Voros [8].

I do not consider motion in bounded domains. For an attempt to calculate wavefunctions and energy levels see Aurich, Sieber and Steiner [7]. To construct the path integral on $\mathcal{H}$, I follow the canonical approach as described in previous papers $[41,42]$. I use the prescription, which I called "product form"-definition. Let us summarize in short the most important facts of this prescription (for details see [38]).

Let us start with the generic case fi.e. the classical Lagrangian is given by $\mathcal{L}_{C l}(q, \dot{q})$ $=\frac{m}{2} g_{a b} \dot{q}^{a} \dot{q}^{b}-V(q)$, the classical Hamiltonian by $\left.\mathcal{H}_{C l}(q, \dot{q})=\frac{m}{2} g^{a b} p_{a} p_{b}+V(q)\right]$ and write the metric tensor $g_{a b}$ in the form (which under reasonable assumptions is always possible, e.g. positive definite scalar product):

$$
g_{a b}(q)=\sum_{c=1}^{d} h_{a c}(q) h_{b c}(q)
$$

( $d=$ dimension of the Riemannian manifold). The quantum Hamiltonian is constructed in the usual way by the Laplace-Beltrami operator $\Delta_{L B}$ (I set $\hbar=1$; in the following sums over repeated indices are implicitely understood)

$$
H=-\frac{1}{2 m} \Delta_{L B}+V(q)=-\frac{1}{2 m} \frac{1}{\sqrt{g}} \frac{\partial}{\partial q^{a}} \sqrt{g} g^{a b} \frac{\partial}{\partial q^{b}}+V(q)
$$

$\left(g=\right.$ determinant of the metric tensor $\left.g_{a b}\right)$. Let us introduce momentum operators $p_{a}=-i\left(\partial_{a}+\Gamma_{a} / 2\right)$, where $\Gamma_{a}=\partial_{a} \ln \sqrt{g}$. Rewriting the Hamiltonian (2) in terms of the momentum operators $p_{a}$ let us choose a product ordering-definition:

$$
H=\frac{1}{2 m} h^{\alpha c}(q) p_{a} p_{b} h^{b c}(q)+V(q)+\Delta V(q)
$$

with the well-defined quantum correction $\Delta V$ giveu by $\left(h:=\operatorname{det}\left(h_{a b}\right)=\sqrt{g}\right)$ :

$$
\begin{aligned}
\Delta Y=\frac{1}{8 m}\left[4 h^{a c} h_{h}^{b c}{ }_{, a b}+2 h^{a c} h^{b c}\right. & \frac{h_{, a b}}{h} \\
& \left.+2 h^{a c}\left(h^{b c}{ }_{, b} \frac{h_{-a}}{h}-h^{b c}{ }_{, a} \frac{h_{, b}}{h}\right)-h^{a c} h^{b c} \frac{h_{, a} h_{, b}}{h^{2}}\right] .
\end{aligned}
$$

Let us assume that $g_{a b}$ is proportional to the unit tensor, i.e. $g_{a b}=f^{2} \delta_{a b}$. Then $\Delta \mathrm{V}$ simplifies into

$$
\Delta Y^{-}=h^{2} \frac{d-2}{8 m f^{4}} \sum_{a=1}^{d}(14-d) f_{a}^{2}-2 f \cdot f_{a a} \vdots
$$

This implies that if the dimension of the space is $d=2$, then the quantum correction $\Delta V$ vanishes.

For details consult Ref. [38]. Using the Trotter formula $e^{-i t(A+B)}=s-\lim _{N \rightarrow \infty}$ $\left(\epsilon^{-i t A / N} e^{-i t B / N}\right)^{N}$ (e.g. [75]) and the short-time approximation for the matrix eiement $\left\langle q^{\prime \prime}\left|e^{-i \epsilon H}\right| q^{\prime}>\right.$ one obtains in the usual manner the Lagrangian path integral in the "product form"-definition $\left[q^{(j)}=q\left(t^{(j)}\right), f^{(j)}=f\left(t^{(j)}\right), t^{(j)}=\right.$ $\left.t^{\prime}+j \epsilon, \epsilon=T / N=\left(t^{\prime \prime}-t^{\prime}\right) / N, N \rightarrow \infty, \Delta q^{(j)}=q^{(j)}-q^{(j-1)}\right]$ :

$$
\begin{aligned}
& K\left(q^{\prime \prime}, q^{\prime} ; T\right)=\int \sqrt{g} D q(t) \exp \left\{i \int_{t^{\prime}}^{t^{\prime \prime}}\left[\frac{m}{2} h_{a c} h_{b c} \dot{q}^{a} \dot{q}^{b}-V(q)-\Delta V(q)\right] d t\right\} \\
:= & \lim _{N \rightarrow \infty}\left(\frac{m}{2 \pi i \epsilon}\right)^{\frac{N d}{2}} \prod_{j=1}^{N-1} \int \sqrt{g\left(q^{(j)}\right)} d q^{(j)} \\
& \times \exp \left\{i \sum_{j=1}^{N}\left[\frac{m}{2 \epsilon} h_{a c}\left(q^{(j-1)}\right) h_{b c}\left(q^{(j)}\right) \Delta q^{a,(j)} \Delta q^{b,(j)}-\epsilon V\left(q^{(j)}\right)-\epsilon \Delta V\left(q^{(j)}\right)\right]\right\}
\end{aligned}
$$

The expression in square brackets is nothing but the classical Lagrangian with an additional quantum correction potential $\Delta V: \mathcal{L}_{e f f}=\mathcal{L}_{C l}-\Delta V$. Clearly, one has to prove that with the short time kernel of this path integral the time-dependent Schrödinger equation

$$
\left[-\frac{1}{2 m} \Delta_{L B}+V(q)\right] \psi(q ; t)=\frac{1}{i} \frac{\partial}{\partial t} \psi(q ; t)
$$

can be derived via the time evolution equation

$$
\psi\left(q^{\prime \prime} ; t^{\prime \prime}\right)=\int \sqrt{g\left(q^{\prime}\right)} K\left(q^{\prime \prime}, q^{\prime} ; T\right) \psi\left(q^{\prime} ; t^{\prime}\right) d q^{\prime} .
$$

This is in fact the case - see [38].

In $\mathcal{H}$ the metric is given by $g_{a b}=\delta_{a b} / y^{2}(x \in \mathbf{R}, y>0)$. The classical Lagrangian and the Hamiltonian read, respectively:

$$
\mathcal{L}_{C l}(x, \dot{x}, y, \dot{y})=\frac{m}{2 y^{2}}\left(\dot{x}^{2}+\dot{y}^{2}\right) . \quad \mathcal{H}_{C l}\left(x, p_{x}, y, p_{y}\right)=\frac{y^{2}}{2 m}\left(p_{x}^{2}+p_{y}^{2}\right)
$$

and the quantum Hamiltonian is given by

$$
H=-\frac{y^{2}}{2 m}\left(\frac{\partial^{2}}{\partial x^{2}} \div \frac{\partial^{2}}{\partial y^{2}}\right)
$$

The scalar product for functions $f_{1}, f_{2} \in L^{2}(\mathcal{H})$ reads,

$$
\left(f_{1}, f_{2}\right)_{\mathcal{H}}=\int_{-\infty}^{\infty} d x \int_{0}^{\infty} \frac{d y}{y^{2}} f_{1}(x, y) \overline{f_{2}}(x, y) .
$$

States $\Psi \in D(H) \cap L^{2}(\mathcal{H})$ must satify the boundary condition $\lim _{y-0} \Psi(x, y)=0(x)$ R). Following the "product-form" prescription, I get for the hermitian momenta:

$$
\begin{array}{ll}
\Gamma_{x}=0, & p_{x}=\frac{1}{i} \frac{\partial}{\partial x} \\
\Gamma_{y}=-\frac{2}{y}, & p_{y}=\frac{1}{i}\left(\frac{\partial}{\partial y}-\frac{2}{y}\right) .
\end{array}
$$


and the Hamiltonian rewritten in the product ordering yiclds:

$$
H=\frac{1}{2 m} y\left(p_{x}^{2}+p_{y}^{2}\right) y \text {. }
$$

Here it has been used that for this special two-dimensional metric: $\Delta V^{*}=0$. Thus we can infer that the path integral on the Poincare upper half-plane in the "product form"-definition reads:

$$
\begin{aligned}
& K^{\mathcal{H}}\left(x^{\prime \prime}, y^{\prime \prime}, x^{\prime}, y^{\prime} ; T\right) \\
& =\lim _{N \rightarrow \infty}\left(\frac{m}{2 \pi i \epsilon}\right)^{N} \prod_{j=1}^{N-1} \int_{-\infty}^{\infty} d x^{(j)} \int_{0}^{\infty} \frac{d y^{(j)}}{y^{(j) 2}} \cdot \exp \left[\frac{i m}{2 \epsilon} \sum_{j=1}^{N} \frac{\Delta^{2} x^{(j)}+\Delta^{2} y^{(j)}}{y^{(j)} y^{(j-1)}}\right] .
\end{aligned}
$$

I want to emphasize that in the "product form"-definition for the path integral on $\mathcal{H}$, there is no additional quantum potential or curvature term. Via the time evolution Eq. (8) it is an easy computation to show that Eq.(14) is indeed the right path integral on $\mathcal{H}$ (see 441 for details).

I now present three alternative ways to calculate the path integral (14).

1) To make the path integral manageable I perform a time-transformation (see [42]):

$$
s(t) \equiv \int_{t^{\prime}}^{t} \frac{1}{f(y(\sigma))} d \sigma, \quad s^{\prime \prime}=s\left(t^{\prime \prime}\right), \quad s\left(t^{\prime}\right)=0
$$

with $f(y)=1 / y^{2}$. The variables $x$ and $y$ are transformed into

$$
\begin{array}{lll}
x(t) \rightarrow \xi(s) & \text { with } & \xi(s(t))=x(t) \\
y(t) \rightarrow \eta(s) & \text { with } & \eta(s(t))=y(t)
\end{array}
$$

with $\xi(0)=x^{\prime}, \xi\left(s^{\prime \prime}\right)=x^{\prime \prime}, \eta(0)=y^{\prime}$ and $\eta\left(s^{\prime \prime}\right)=y^{\prime \prime}$. Let us assume that the constrain

$$
\int_{0}^{s^{\prime \prime}} \frac{d s}{\eta^{2}(s)}=T
$$

has for all admissible paths a unique solution $s^{\prime \prime}>0$. Of course, since $T$ is fixed, the "time" $s$ " will be path-dependent. To incorporate the constraint (1i) the identity

$$
\begin{aligned}
1=\frac{1}{y^{\prime \prime 2}} \int_{0}^{\infty} d s^{\prime \prime} \delta\left(\int_{0}^{s^{\prime \prime}}\right. & \left.\frac{d s}{\eta^{2}(s)}-T\right) \\
& =\frac{1}{y^{\prime \prime 2}} \int_{-\infty}^{\infty} \frac{d E}{2 \pi} \epsilon^{-i T E} \int_{0}^{\infty} d s^{\prime \prime} \exp \left(i \int_{0}^{s^{\prime \prime}} d s \frac{E}{\eta^{2}(s)}\right)
\end{aligned}
$$

has to be used in the path integral (14). The only difference to the prescription given in [42] is that we have now only a time- and not a space-time-transformation. This has the consequence that the additional factor in equation (IV.6) of [42] is absent in the present case. Defining the energy-dependent Feynman kernel $G(E)$ via the Fourier transformation ${ }^{1}$

$$
K^{\mathcal{H}}\left(x^{\prime \prime}, y^{\prime \prime}, x^{\prime}, y^{\prime} ; T\right)=\frac{1}{2 \pi i} \int_{-\infty}^{\infty} e^{-i T E} G^{\mathcal{H}}\left(x^{\prime \prime}, y^{\prime \prime}, x^{\prime}, y^{\prime} ; E\right) d E
$$

${ }^{1}$ To work with well-defined mathematical formulas let us assume that $E$ has a small positive imaginary part if, and write $E+i \epsilon$ (with real $E$ ) instead of $E$ whenever necessary. Also, square roots will be positive. See e.g. $[39,46]$ for details.
I obtain the transformation formula

$$
G^{\mathcal{H}}\left(x^{\prime \prime}, y^{\prime \prime}, x^{\prime}, y^{\prime} ; E\right)=i \int_{0}^{\infty} \bar{K}\left(\xi^{\prime \prime}, \eta^{\prime \prime}, \xi^{\prime}, \eta^{\prime} ; s^{\prime \prime}\right) d s^{\prime \prime},
$$

where the transformed path integral is given by

$$
\begin{aligned}
& \tilde{K}\left(\xi^{\prime \prime}, \eta^{\prime \prime}, \xi^{\prime}, \eta^{\prime}: s^{\prime \prime}\right)=\int D \xi(s) \mu_{\lambda}\left[\eta_{i}^{\prime} D \eta(s) \exp \left[\frac{i m}{2} \int_{0}^{s}\left(\dot{\xi}^{2}+\dot{\eta}^{2}\right) d s\right]\right. \\
&=\lim _{N^{\prime} \rightarrow \infty}\left(\frac{m}{2 \pi i \delta}\right)^{N} \int_{-\infty}^{\infty} \int_{0}^{\infty} d \xi_{(1)} d \eta_{(1)} \cdots \int_{-\infty}^{\infty} \int_{0}^{\infty} d \xi_{(N-1)} d \eta_{(N-1)} \\
& \times \mu_{\lambda}\left\{\eta_{(j)}\right\} \exp \left\{\frac{i m}{2 \delta} \sum_{j=1}^{N}\left[\left(\xi_{(j)}-\xi_{(j-1)}\right)^{2}+\left(\eta_{(j)}-\eta_{(j-1)}\right)^{2}\right]\right\}
\end{aligned}
$$

with $\delta=s^{\prime \prime} / \Lambda^{\prime}$ and $\lambda=\sqrt{1 / 4-2 m E}$. The functional measure is given by

$$
\mu_{\lambda}[\eta] \rightarrow \prod_{j=1}^{N}\left[\sqrt{\frac{2 \pi m}{i \delta} \eta_{(j)} \eta_{(j-1)}} \exp \left(-\frac{m}{i \delta} \eta_{(j)} \eta_{(j-1)}\right) I_{\lambda}\left(\frac{m}{i \delta} \eta_{(j)} \eta_{(j-1)}\right)\right] .
$$

$I_{\lambda}$ denotes a modified Bessel function. Following the general theory [42], it has beez used:

$$
g_{a b}=\delta_{a b}, \quad \sqrt{g}=1, \quad \Gamma_{\xi}=0, \quad \Gamma_{\eta}=0, \quad \Delta V=0 .
$$

The path integral in (21) factorizes into a path integral for a free particle in $\xi \in \mathbf{R}$ and into a radial path integral with "angular momentum" $\lambda$ in the variable $\eta \in \mathbf{R}^{\perp}$. Using the well-known path integral identity

$$
\int \mu_{\lambda}\left[r \mid D r(t) \exp \left(\frac{i m}{2} \int_{t^{\prime}}^{t^{\prime \prime}} \dot{r}^{2} d t\right)=\sqrt{r^{\prime} r^{\prime \prime}} \frac{m}{i T} \exp \left(\frac{i m}{2 T}\left(r^{\prime 2}+r^{\prime \prime 2}\right)\right) I_{\lambda}\left(\frac{m}{i T} r^{\prime} r^{\prime \prime}\right)\right.
$$

(see Peak and Inomata [69] and Steiner and Grosche [41j). we can immediately write down the solution of $(21)$ :

$$
\begin{aligned}
& \tilde{K^{\prime}}\left(\xi^{\prime \prime}, \eta^{\prime \prime}, \xi^{\prime}: \eta^{\prime}: s^{\prime \prime}\right) \\
& \left.\quad=\sqrt{\frac{\eta^{\prime} \eta^{\prime \prime}}{2 \pi}}\left(\frac{m}{i s^{\prime \prime}}\right)^{3 / 2} \exp \left\{-\frac{m}{2 i s^{\prime \prime}} i\left(\xi^{\prime \prime}-\xi^{\prime}\right)^{2}+\eta^{\prime \prime 2}+\eta^{\prime 2}\right]\right\} I_{\lambda}\left(\frac{m}{i s^{\prime \prime}} \eta^{\prime} \eta^{\prime \prime}\right) .
\end{aligned}
$$

Inserting (25) into Eq.(20), the $s^{\prime \prime}$-integration can be carried out by first performing a Feynman-Wick rotation $\left(s^{\prime \prime} \rightarrow-i \tau, \tau \in \mathbf{R}^{+}\right)$, and then introducing the integration variable $z=m y^{\prime} y^{\prime \prime} / \tau$ and the Poincare distance cosh $d\left(z^{\prime \prime}, z^{\prime}\right) \equiv\left[\left(x^{\prime \prime}-x^{\prime}\right)^{2}+y^{\prime 2}+\right.$ $\left.y^{\prime \prime 2}\right] / 2 y^{\prime} y^{\prime \prime}$. I obtain (for the integral see p.712 of reference [33]):

$$
G^{\mathcal{H}}\left(x^{\prime \prime}, y^{\prime \prime}, x^{\prime}, y^{\prime} ; E\right)=\frac{m}{\sqrt{2 \pi}} \int_{0}^{\infty} \epsilon^{-z \cosh d} I_{i p}(z) \frac{d z}{\sqrt{z}}=\frac{m}{\pi} \mathcal{Q}_{-\frac{1}{2}-i p}(\cosh d),
$$

where I have introduced the momentum $p \equiv \sqrt{2 m E-1 / 4}$. Eq.(26) gives a closed expression for the energy-dependent Green's function (resolvent kernel) in terms of the 
Legendre function of the second kind $\mathcal{Q}_{\nu}{ }^{1}$. This result agrees with the one obtained by solving directly the Schrödinger equation (see e.g. [46]). Using the integrals (see [33], pp.819, 732):

$$
\begin{aligned}
& \mathcal{Q}_{\nu-\frac{1}{2}}\left(\frac{a^{2}+b^{2}+c^{2}}{2 a b}\right)=\int_{0}^{\infty} d p^{\prime} \frac{p^{\prime} \tanh \pi p^{\prime}}{\nu^{2}+p^{\prime 2}} \mathcal{P}_{i p^{\prime}-\frac{1}{2}}\left(\frac{a^{2}+b^{2}+c^{2}}{2 a b}\right) \\
& \mathcal{P}_{\nu-\frac{1}{2}}\left(\frac{a^{2}+b^{2}+c^{2}}{2 a b}\right)=\frac{4 \sqrt{a b}}{\pi^{2}} \cos \nu \pi \int_{0}^{\infty} d k K_{\nu}(a k) K_{\nu}(b k) \cos c k
\end{aligned}
$$

Eq.(26) can be rewritten as

$$
\begin{aligned}
& G^{\mathcal{H}}\left(x^{\prime \prime}, y^{\prime \prime}, x^{\prime}, y^{\prime} ; E\right) \\
= & \frac{1}{\pi^{3}} \int_{-\infty}^{\infty} d k \int_{0}^{\infty} d p^{\prime} \frac{p^{\prime} \sinh \pi p^{\prime}}{\left(p^{\prime 2}+\frac{1}{4}\right) / 2 m-E} \sqrt{y^{\prime} y^{\prime \prime}} K_{i p^{\prime}}\left(|k| y^{\prime}\right) K_{i p^{\prime}}\left(|k| y^{\prime \prime}\right) e^{i k\left(x^{\prime \prime}-x^{\prime}\right)}
\end{aligned}
$$

( $K_{\nu}$ denotes a modified Bessel function). The representation (28) shows clearly that $G(E)$ has a cut on the positive real axis in the complex energy plane with a branch point at $E=1 / 8 \mathrm{~m}$. We thus infer that the quantum mechanical motion on the Poincaré upper half-plane $\mathcal{H}$ has a continuous energy spectrum. Fron $(28)$ the normalized wave functions and the energy spectrum can be read off:

$$
\left.\begin{array}{rl}
\psi_{p, k}(x, y) & =\sqrt{\frac{p \sinh \pi p}{\pi^{3}}} e^{i k x} \sqrt{y} K_{i p}(|k| y) \quad(x \in \mathbf{R}, y>0) \\
E_{p} & =\frac{1}{2 m}\left(p^{2}+\frac{1}{4}\right)
\end{array}\right\}
$$

with $p>0$ and $k \in \mathbf{R} \backslash\{0\}$. These are the correct wave functions. The spec trum has a largest lower bound $E_{0}=1 / 8 \mathrm{~m}$. A state with $p=0$ and $E_{0}=1 / 8 \mathrm{~m}$ does not exist, because $x_{0, k}$ vanishes identically. One also has to exclude the case $k=0$, which is obvious from the asymptotic behaviour of the $K_{\nu}$ function for $z \rightarrow 0$ $K_{i p}(z) \rightarrow \frac{1}{2}\left[\Gamma(i p)\left(\frac{2}{z}\right)^{i p}+\Gamma(-i p)\left(\frac{2}{2}\right)^{-i p}\right]$. It is nevertheless possible to define a func. tion $\dot{\phi}_{p}(y):=y^{i p+1 / 2}$ which is an Eigenstate of $\mathrm{H}, H \phi_{p}=E_{p} \phi_{p}$, but this function is not normalizable in $\mathcal{H} . \phi_{p}$ is only normalizable in a bounded domain. Let us discus in short the "zero-momentum" energy shift $E_{0}=\frac{1}{8 m}$. Let us consider the classical Hamiltonian $\mathcal{H}_{C l}$ and insert (introducing $\hbar$ ) the Heisenberg uncertainty relations $x \cdot p_{x} z \hbar / 2$ and $y p_{y} \geq \hbar / 2$. This gives for the energy of quantum motion on $\mathcal{H}$ the lower bound:

$$
E_{\mathcal{H}} \geq \frac{\hbar^{2}}{8 m}\left(1+\frac{y^{2}}{x^{2}}\right)>\frac{\hbar^{2}}{8 m} .
$$

The value $E_{0}=\inf E_{\mathcal{H}}=\frac{\hbar^{2}}{8 m}$ can never be taken on because $\{z \mid y=0\} \notin \mathcal{H} . E_{0}$ is the largest lower bound on $\mathcal{H}$.

On the pseudosphere $\Lambda^{2}$, the Poincare disc $D$ and the hyperbolic strip $S$ the

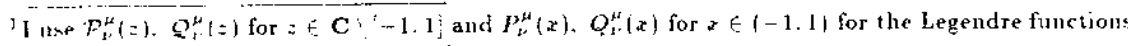
if the first and serond kind, respectively. wave functions are given by $(p>0, l \in \mathbf{Z}, k \in \mathbf{R})$ :

$$
\left.\begin{array}{rl}
\Psi_{p, l}^{\Lambda^{2}}(\tau, \phi) & =\sqrt{\frac{p \sinh \pi p}{2 \pi^{2}}} \Gamma(i p+l+1 / 2) \mathcal{P}_{i p-\frac{1}{2}}^{-1}(\cosh \tau) e^{i l \phi} \\
\Psi_{p, l}^{D}(r, \psi) & =\sqrt{\frac{p \sinh \pi p}{2 \pi^{2}}} \Gamma\left(\frac{1}{2}+i p+l\right) \mathcal{P}_{i p-\frac{1}{2}}^{-l}\left(\frac{1+r^{2}}{1-r^{2}}\right) e^{i l \psi} \\
\Psi_{p, k}^{S}(X, Y) & =\sqrt{\frac{p \sinh \pi p}{4 \pi\left(\cosh ^{2} \pi k+\sinh ^{2} \pi p\right)}} \sqrt{\cos Y} P_{i k-\frac{1}{2}}^{i p}(\sin Y) e^{i k X} .
\end{array}\right\}
$$

The corresponding path integral expressions on $\mathcal{H}, \Lambda^{2} D$ and $S$ are equivalent to each other. This has been discussed in detail in Refs. [40,43]; see also the end of this section.

Finally, we perform a Fourier transformation in (28) to get the time-dependent Feynman kernel

$$
\begin{aligned}
& K^{\mathcal{H}}\left(x^{\prime \prime}, y^{\prime \prime}, x^{\prime}, y^{\prime} ; T\right) \\
= & \frac{1}{\pi^{3}} \int_{-\infty}^{\infty} d k \int_{0}^{\infty} d p p \sinh \pi p e^{-i T^{p^{2}+1 / 4} \frac{1 / 4}{2 m}} \sqrt{y^{\prime} y^{\prime \prime}} K_{i p}\left(|k| y^{\prime}\right) K_{i p}\left(|k| y^{\prime \prime}\right) e^{i k\left(x^{\prime \prime}-x^{\prime}\right) .}
\end{aligned}
$$

The $\psi_{p, k}$ form an orthonormal basis

$$
\int_{-\infty}^{\infty} d x \int_{0}^{\infty} \frac{d y}{y^{2}} \bar{\psi}_{p, k}(x, y) \psi_{p^{\prime}, k^{\prime}}(x, y)=\delta\left(k-k^{\prime}\right) \delta\left(p-p^{\prime}\right)
$$

Proof: Inserting $\psi_{p, k}$ from Eq.(29) and performing the $x$-integration yields:

$$
N=\delta\left(k-k^{\prime}\right) \frac{2 \sqrt{p p^{\prime} \sinh \pi p} \sinh p^{i}}{\pi^{2}} \int_{0}^{\infty} \frac{1}{y} K_{i p}(y) K_{i p^{\prime}}(y) d y .
$$

Using the integral ([33], p.693):

$$
\begin{aligned}
& \int_{0}^{\infty} y^{-\lambda} K_{\mu}(a y) K_{\nu}(b y) d y=\frac{a^{\lambda-\nu-1} b^{\nu}}{2^{2+\lambda} \Gamma(1-\lambda)} \\
& \times \Gamma\left(\frac{1-\lambda+\mu-\nu}{2}\right) \Gamma\left(\frac{1-\lambda-\mu+\nu}{2}\right) \Gamma\left(\frac{1-\lambda+\mu-\nu}{2}\right) \Gamma\left(\frac{1-\lambda-\mu-\nu}{2}\right) \\
& \times F\left(\frac{1-\lambda+\mu+\nu}{2}, \frac{1-\lambda-\mu-\nu}{2}: 1-\lambda: 1-\frac{b^{2}}{a^{2}}\right) .
\end{aligned}
$$

Let $a=b=1, \lambda=1-2 \epsilon, \mu=i p$ and $\nu=i p+2 i q . q=\left(p^{\prime}-p\right) / 2$, thell

$$
\int_{0}^{\infty} y^{2 \epsilon-1} K_{i p}(y) K_{i p+2 i q}(y) d y=\frac{\Gamma(\epsilon+i p+i q) \Gamma(\epsilon-i q) \Gamma(\epsilon-i q) \Gamma(\epsilon-i p-i q)}{\Gamma(2 \epsilon) 2^{3-2 \epsilon}}
$$

The "good" terms yield in the limit $\epsilon \rightarrow 0$

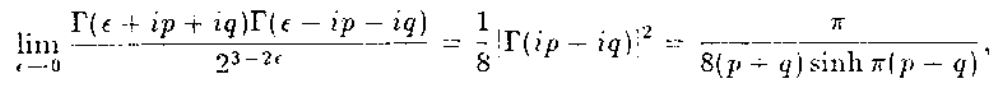


where I have used a well-know property of the $\Gamma$-function. The remaining ferms vield

$$
\lim _{\epsilon \rightarrow 0} \frac{\Gamma(\epsilon+i q) \Gamma(\epsilon-i q)}{\Gamma(2 \epsilon)}=2 \pi \lim _{\epsilon \rightarrow 0} \frac{\epsilon}{\pi\left(\epsilon^{2}+q^{2}\right)}=4 \pi \delta\left(p^{\prime}-p\right)
$$

and $\mathrm{Eq} .(33)$ is proved.

Vice versa, the $\psi_{p, k}$ form a complete set, i.e.

$$
\int_{-\infty}^{\infty} d k \int_{0}^{\infty} d p \psi_{p, k}^{\prime}\left(x^{\prime \prime}, y^{\prime \prime},\right) \bar{\psi}_{p, k}\left(x^{\prime}, y^{\prime}\right)=y^{\prime} y^{\prime \prime} \delta\left(x^{\prime \prime}-x^{\prime}\right) \delta\left(y^{\prime \prime}-y^{\prime}\right)
$$

(the factor $y^{\prime} y^{\prime \prime}=\left(g^{\prime} g^{\prime \prime}\right)^{-\frac{1}{4}}$ has to be included due to the Riemannian structure of $\mathcal{H}$, see e.g. $[6 \tau])$.

Proof: Consider the integral ([33) p.772):

$$
\int_{0}^{\infty} d x K_{i x}(a) K_{i x}(b) \cosh [(\pi-\phi) x]=h_{0}\left(\sqrt{a^{2}+b^{2}-2 a b \cos \phi}\right) .
$$

Differentiation with respect to $\phi$ gives on the left hand side:

$$
-\frac{\partial}{\partial \phi} \int_{0}^{\infty} d x K_{i x}(a) K_{i x}(b) \cosh [(\pi-\phi) x]=\int_{0}^{\infty} d x x \sinh [(\pi-\phi) x] K_{i x}(a) K_{i x}(b),
$$

while the right hand side yieids:

$$
-\frac{\partial}{\partial \phi} K_{0}\left(\sqrt{a^{2}+b^{2}-2 a b \cos \phi}\right)=\frac{a b \sin \phi}{\sqrt{a^{2}+b^{2}-2 a b \cos \phi}} K_{1}\left(\sqrt{a^{2}+b^{2}-2 a b \cos \phi}\right) .
$$

Here some properties of the $K_{\nu}$-function have been used (see e.g. [33], p.510). Therefore in the limit $\phi \rightarrow 0$ and for $y^{\prime} \neq y^{\prime \prime}$ :

$$
\int_{0}^{\infty} d p p \sinh \pi p K_{i p}^{-}\left(|k| y^{\prime}\right) K_{i p}\left(|k| y^{\prime \prime}\right)=0 \text {. }
$$

It remains to consider the case $y^{\prime} \simeq y^{\prime \prime}$. Let us set $y=y^{\prime}, y^{\prime \prime}=y+\delta$ with $\mid \delta: \leqslant 1$ and $\cos \phi \simeq 1-\phi^{2} / 2$ for $|\phi| \ll 1$. Using $K_{0} \simeq-\ln (z / 2)(z \rightarrow 0)$ we get for the right hand side of Eq. $(40)$ :

$$
\frac{\pi}{2} K_{0}\left(|k| \sqrt{y^{\prime 2}+y^{\prime \prime 2}-2 y^{\prime} y^{\prime \prime} \cos \phi}\right) \simeq \frac{\pi}{2}\left[\ln \frac{|k|}{2}+\frac{1}{2} \ln \left(\delta^{2}+y^{2} \phi^{2}\right)\right] \quad(|\delta|,|\phi| \ll 1)(44)
$$

and in the limit $\phi \rightarrow 0$ :

$$
\int_{0}^{\infty} d p p \sinh \pi p K_{i p}\left(|k| y^{\prime}\right) K_{i p}^{r}\left(|k| y^{\prime \prime}\right)=\frac{\pi^{2}}{2} \sqrt{y^{\prime} y^{\prime \prime}} \delta\left(y^{\prime}-y^{\prime \prime}\right) .
$$

Together with the well-known equation $\frac{1}{2 \pi} \int_{-\infty}^{\infty} d k \epsilon^{i k\left(x^{\prime \prime}-x^{\prime}\right)}=\delta\left(x^{\prime \prime}-x^{\prime}\right)$ the completeness relation (39) is proven.
2) In the second approach in calculating the path integral (14) - following the idea of Kubo 54 - let us start by integrating $h^{\prime}(T)$ over $x^{\prime \prime} \equiv x^{(N)}$. This gives:

$$
\begin{gathered}
\int_{-\infty}^{\infty} K^{-\mu}\left(x^{\prime \prime}, y^{\prime \prime}, x^{\prime}, y^{\prime} ; T\right) d x^{\prime \prime}=\lim _{V_{\cdots \infty}}\left(\frac{m}{2 \pi i \epsilon}\right)^{N} \prod_{j=1}^{N-1} \int_{0}^{\infty} \frac{d y^{(j)}}{y^{(j) 2}} \\
\times \exp \left[\frac{i m}{2 \epsilon} \sum_{j=1}^{N} \frac{\Delta^{2} y^{(j)}}{y^{(j)} y^{(j-1)}}\right] \cdot \prod_{j=1}^{N} \int_{-\infty}^{\infty} d x^{(j)} \exp \left(-\frac{m}{2 i \epsilon} \frac{\Delta^{2} x^{(j)}}{y^{(j)} y^{(j-1)}}\right) \\
=\sqrt{y^{\prime} y^{\prime \prime}} \lim _{n \rightarrow \infty}\left(\frac{m}{2 \pi i \epsilon}\right)^{\frac{N}{2}} \prod_{j=1}^{N-1} \int_{0}^{\infty} \frac{d y^{(j)}}{y^{(j)}} \exp \left[\frac{i m}{2 \epsilon} \sum_{j=1}^{N} \frac{\Delta^{2} y^{(j)}}{y^{(j)}} y^{(j-1)}\right] \equiv \hat{K}\left(y^{\prime \prime}, y^{\prime} ; T\right) .
\end{gathered}
$$

Let us look for a transformation $z=z(y)$ so that

$$
\dot{i}^{2}=\frac{\dot{y}^{2}}{y^{2}} \text {. }
$$

A simple calculation yields $z=\ln y$, respectively the inverse transformation $y=\epsilon^{z}$. $z(y)$ las the property $(0, \infty) \mapsto \mathbf{R}$. Thus $d y / y=d z$, and the kinetic term in the exponential in the path integral (46) gives in a Taylor expansion:

$$
\frac{\Delta^{2} y^{(j)}}{y^{(j)} y^{(j-1)}}=\epsilon^{\Delta z^{(j)}-2 z^{(j)}}\left[e^{z^{(j)}}-\epsilon^{z^{(j)}-\Delta z^{(j)}}\right]^{2} \simeq \Delta^{2} z^{(j)}+\frac{\Delta^{4} z^{(j)}}{12} .
$$

Exploiting the path integral identity $\Delta^{4} \dot{z}=3\left(\frac{i m}{\epsilon}\right)^{2}$ I get: ${ }^{1}$

$$
\exp \left(\frac{i m}{2 \epsilon} \cdot \frac{\Delta^{2} y^{(j)}}{y^{(j)} y^{(j-1)}}\right) \doteq \exp \left(\frac{i m}{2 \epsilon} \Delta^{2} z^{(j)}-\frac{i \epsilon}{8 m}\right) .
$$

Therefore the path integral (46) gives essentially a path integral of a free particle in $\mathbf{R}$ in the $z$-coordinate. We get with $K^{\prime}\left(z^{\prime \prime}, z^{\prime} ; T\right) \equiv K^{\prime}\left(y^{\prime \prime}, y^{\prime} ; T\right)$ :

$$
\begin{aligned}
& \dot{K}\left(z^{\prime \prime} . z^{\prime}: T\right) \\
& =\exp \left[\frac{z^{\prime}+z^{\prime \prime}}{2} \cdot \frac{i T}{8 m}\right] \lim _{N \rightarrow \infty}\left(\frac{m}{2 \pi i \epsilon}\right)^{\frac{N}{2}} \prod_{j=1}^{N-1} \int_{-\infty}^{\infty} d z^{(j)} \exp \left(\frac{i m}{2 \epsilon} \sum_{j=1}^{N} \Delta^{2} z^{(j)}\right) \\
& =\exp \left[\dot{z}^{\prime}+\frac{z^{\prime \prime}}{2}-\frac{i T}{8 m}\right]\left(\frac{m}{2 \pi i T}\right)^{\frac{1}{2}} \exp \left[\frac{i m}{2 T}\left(z^{\prime \prime}-z^{\prime}\right)^{2}\right] \\
& \quad-\epsilon^{-i T / 8 m} \sqrt{y^{\prime} y^{\prime \prime}}\left(\frac{m}{2 \pi i T}\right)^{\frac{1}{2}} \exp \left[-\frac{m}{2 i T} \ln ^{2}\left(\frac{y^{\prime \prime}}{y^{\prime}}\right)\right]=\tilde{K}^{\prime}\left(y^{\prime \prime}, y^{\prime} ; T\right) . \quad(50)
\end{aligned}
$$

Let us note that $l=\ln \left(y^{\prime \prime} / y^{\prime}\right)$ is nothing but the hyperbolic distance $d$ in $\mathcal{H}$ if $\left(x^{\prime \prime}-x^{\prime}\right)=0$. Introducing now

$$
k:=\frac{\xi^{2}+y^{\prime 2}+y^{\prime \prime 2}}{2 y^{\prime} y^{\prime \prime}}
$$

${ }^{1}$ I use the symbol $\risingdotseq$ (following DeWit1 [21) to denote "equivalence as far as use in the path integral is concerned". A discussion concerning these identities can be, e.g. found in Feynman and Hibbs [27]; see also :42] and references therein. 
I obtain

$$
\bar{K}\left(y^{\prime \prime}, y^{\prime} ; T\right)=\int_{l}^{\infty} K\left(k, y^{\prime \prime}, y^{\prime} ; T\right) \frac{d k}{\sqrt{k-l}}=\left(\frac{m}{2 \pi i T}\right)^{\frac{1}{2}} \exp \left(-\frac{m}{2 i T} \operatorname{arcosh}^{2} l-\frac{i T}{8 m}\right) .
$$

This integral equation can be solved exactly and the result reads (see [54]):

$$
K^{\mathcal{H}}(d ; T)=\sqrt{2}\left(\frac{m}{2 \pi i T}\right)^{\frac{2}{2}} \int_{d}^{\infty} \frac{u d u}{\sqrt{\cosh u-\cosh d}} \exp \left[-\frac{m}{2 i T} u^{2}-\frac{i T}{8 m}\right] .
$$

Fourier transformation to get the Green's function $G(E)=\int_{0}^{\infty} \epsilon^{i T E} K(T) d T$ yields:

$$
\begin{aligned}
& G^{\mathcal{H}}(d ; E)=\sqrt{2}\left(\frac{m}{2 \pi i}\right)^{\frac{5}{2}} \int_{d}^{\infty} \frac{u d u}{\sqrt{\cosh u-\cosh d}} d u \\
& \quad \times \int_{0}^{\infty} T^{-\frac{5}{2}} \exp \left[-\frac{m}{2 i T} u^{2}-\left(\frac{i}{8 m}-i E\right) T\right] d T \\
& =\frac{m}{\pi \sqrt{2}} \int_{d}^{\infty} \frac{\exp \left(-i u \sqrt{2 m E-\frac{1}{4}}\right)}{\sqrt{\cosh u} \cdots \cosh d} d u=\frac{m}{\pi} \mathcal{Q}_{-\frac{1}{2}-i \sqrt{2 m E-\frac{1}{4}}}(\cosh d) .
\end{aligned}
$$

This is the previous result. Note that $K^{\mathcal{H}}$ and $G^{\mathcal{H}}$ are only a function of the hyperbolic distance $d$. Therefore Eqs.(53) and (54) gives the result in all the four spaces $\mathcal{H}, D$, $\Lambda^{2}$ and $S$.

3) In the third approach in calculating the path integral (14) let us start by performing a Fourier expansion of $K^{\mathcal{H}}(T)$

$$
\begin{gathered}
K^{\mathcal{H}}\left(x^{\prime \prime}, y^{\prime \prime}, x^{\prime}, y^{\prime} ; T\right)=\int_{-\infty}^{\infty} K_{k}\left(y^{\prime \prime}, y^{\prime} ; T\right) \epsilon^{i k\left(x^{\prime \prime}-x^{\prime}\right)} d k \\
K_{k}\left(y^{\prime \prime}, y^{\prime} ; T\right)=\frac{1}{2 \pi} \int_{-\infty}^{\infty} K^{-\mathcal{H}}\left(x^{\prime \prime}, y^{\prime \prime}, x^{\prime}, y^{\prime} ; T\right) \epsilon^{-i k\left(x^{\prime \prime}-x^{\prime}\right)} d x^{\prime \prime} .
\end{gathered}
$$

This gives if $\mathrm{Eq} .(14)$ is inserted into (55) for $K_{k}^{-\mathcal{H}}(T)$ :

$$
\begin{aligned}
& K_{k}\left(y^{\prime \prime}: y^{\prime} ; T\right)=\frac{1}{2 \pi} \lim _{N^{\prime} \rightarrow \infty}\left(\frac{m}{2 \pi i \epsilon}\right)^{N} \prod_{j=1}^{N-3} \int_{0}^{\infty} \frac{d y^{(j)}}{y^{(j) 2}} \exp \left[\frac{i m}{2 \epsilon} \sum_{j=1}^{N} \frac{\Delta^{2} y^{(j)}}{y^{(j)} y^{(j-1)}}\right] \\
& \times \prod_{j=1}^{N} \int_{-\infty}^{\infty} d x^{(j)} \exp \left[-\frac{m}{2 i \epsilon} \frac{\Delta^{2} x^{(j)}}{y^{(j)} y^{(j-1)}}-i k \Delta x^{(j)}\right] \\
& =\frac{\sqrt{y^{\prime} y^{\prime \prime}}}{2 \pi} \lim _{N^{\prime}-\infty}\left(\frac{m}{2 \pi i \epsilon}\right)^{\frac{N}{2}} \prod_{j=1}^{N-1} \int_{0}^{\infty} \frac{d y^{(j)}}{y^{(j)}} \exp \left\{i \sum_{j=1}^{N}\left[\frac{m}{2 \epsilon} \frac{\Delta^{2} y^{(j)}}{y^{(j)} y^{(j-1)}}-\epsilon \frac{k^{2} y^{(j)} y^{(j-1)}}{2 m}\right]\right\} \\
& =\frac{\sqrt{y^{\prime} y^{\prime \prime}}}{2 \pi} \int \frac{D y(t)}{y} \exp \left[i \int_{t^{\prime}}^{t^{\prime \prime}}\left(\frac{m}{2} \frac{\dot{y}^{2}}{y^{2}}-\frac{k^{2} y^{2}}{2 m}\right) d t\right] .
\end{aligned}
$$

Performing the transformation $z=\ln y, y=c^{z}$ in (56) and repeating the same procedure as in Eq.(46) I get with $K_{k}\left(z^{\prime \prime}, z^{\prime} ; T\right) \equiv K_{k}\left(y^{\prime \prime}, y^{\prime} ; T\right)$ :

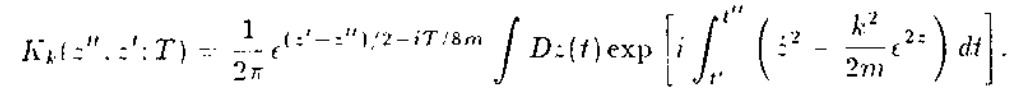

This path integral is nothing but a path integral for the potential of Liouville quantum mechanics with the potential $V(z)=\frac{k^{2}}{2 m} \epsilon^{2 z}$. This path integral was calculated by Steiner and Grosche [41] and the result for $\hat{K}_{k}$ therefore reads,

$\hat{K}_{k}\left(z^{\prime \prime}, z^{\prime} ; T\right)$

$$
=\frac{1}{\pi^{3}} e^{\left(z^{\prime}+z^{\prime \prime}\right) / 2} \int_{0}^{\infty} d p p \sinh \pi p \exp \left[-\frac{i T}{2 m}\left(p^{2}+\frac{1}{4}\right)\right] K_{i p}\left(|k| e^{z^{\prime}}\right) K_{i p}\left(|k| e^{z^{\prime \prime}}\right) .
$$

Inserting $y=e^{z}$, this gives finally for the Feynman kernel on $\mathcal{H}$

$$
\begin{aligned}
K^{\mathcal{H}}\left(x^{\prime \prime}, y^{\prime \prime}, x^{\prime}, y^{\prime} ; T\right) & =\frac{1}{\pi^{3}} \int_{-\infty}^{\infty} d k \int_{0}^{\infty} d p p \sinh \pi p \\
\times & \exp \left[-\frac{i T}{2 m}\left(p^{2}+\frac{1}{4}\right)\right] \sqrt{y^{\prime} y^{\prime \prime}} K_{i p}\left(|k| y^{\prime}\right) K_{i p}\left(|k| y^{\prime \prime}\right) \epsilon^{i k\left(x^{\prime \prime}-x^{\prime}\right)}
\end{aligned}
$$

with the correct energy-spectrum and wave-functions as in (29).

Using now the integral repesentation ([33], p.732):

$$
\int_{0}^{\infty} K_{\nu}(a x) K_{\nu}(b x) \cos c x d x=\frac{\pi^{2}}{4 \sqrt{a b} \cos \nu \pi} \mathcal{P}_{-\frac{1}{2}+\nu}\left(\frac{a^{2}+b^{2}+c^{2}}{2 a b}\right),
$$

and the addition theorem for the associated Legendre functions: ([33], p.1014)

$$
\mathcal{P}_{\nu}\left(z z^{\prime}-\sqrt{z^{2}-1} \sqrt{z^{\prime 2}-1} \cos \phi\right)=\sum_{l=-\infty}^{\infty}(-1)^{l} e^{i l \phi} \frac{\Gamma(\nu-l+1)}{\Gamma(\nu+l+1)} \mathcal{P}_{\nu}^{l}(z) \mathcal{P}_{\nu}^{l}\left(z^{\prime}\right)
$$

the identity:

$$
\begin{aligned}
& \frac{1}{\pi^{3}} \int_{-\infty}^{\infty} d k \int_{0}^{\infty} d p p \sinh \pi p e^{-\frac{i \gamma}{2 m}\left(p^{2}+\frac{1}{4}\right)} \sqrt{y^{\prime} y^{\prime \prime}} K_{i p}\left(|k| y^{\prime}\right) K_{i p}\left(|k| y^{\prime \prime}\right) \epsilon^{i k\left(x^{\prime \prime}-x^{\prime}\right)} \\
= & \frac{1}{2 \pi^{2}} \sum_{l=-\infty}^{\infty} \int_{0}^{\infty} d p p \sinh \pi p e^{-\frac{i T}{2 m}\left(p^{2}+\frac{1}{4}\right)}\left|\Gamma\left(\frac{1}{2}+i p-l\right)\right|^{2} \\
\times & \times \epsilon^{i l\left(\phi^{\prime \prime}-\phi^{\prime}\right)} \mathcal{P}_{-\frac{1}{2}+i p}^{l}\left(\cosh \tau^{\prime}\right) \mathcal{P}_{-\frac{1}{2}+i p}^{\prime}\left(\cosh \tau^{\prime \prime}\right) . \quad \text { (62) }
\end{aligned}
$$

can be derived. Here use has been made of Eq.(I.14). The right hand side of Eq.(62) represents the Feynnaan kernel on $\Lambda^{2}$ and thus this shows the equivalence of the Feynman-kernels on $\mathcal{H}$ and $\Lambda^{2}$, i.e. $K^{-H}(T) \cong K^{-\Lambda^{2}}(T)$. Inserting on the right hand side of Eq.(62) the variables of the Dise $D$ I get the Feynamn kernel on $D$ :

$$
\begin{aligned}
& K^{D}\left(r^{\prime \prime}, r^{\prime}, \dot{\zeta}^{\prime \prime}, \psi^{\prime} ; T\right)=\frac{1}{2 \pi^{2}} \int_{0}^{\infty} d p \sum_{l=-\infty}^{\infty} p \sinh \pi p \exp \left[-\frac{i T}{2 m}\left(p^{2}-\frac{1}{4}\right)\right] \\
& \times: \Gamma\left(\frac{1}{2}+i p \cdot l_{i}^{\prime 2} \mathcal{P}_{i p-\frac{1}{2}}^{-l}\left(\frac{1+r^{\prime 2}}{1-r^{\prime 2}}\right) \mathcal{P}_{i p-\frac{1}{2}}^{-1}\left(\frac{1+r^{\prime \prime 2}}{1 \cdots r^{\prime \prime 2}}\right) \epsilon^{i l\left(r^{\prime \prime \prime}-\psi^{\prime \prime}\right)}\right.
\end{aligned}
$$

There is no obvious simple manipulation in, e.g. Eq.(63) to achieve the Feymman kerne on the hyperbolic strip $S$. One has to calculate $K^{-S}$ directly. This has been done in Ref. 40 and the result reads:

$$
\begin{aligned}
& K^{-S}\left(X^{\prime \prime}, X^{\prime}, Y^{\prime \prime}, Y^{-1} ; T\right)=\frac{1}{4 \pi} \int_{-\infty}^{\infty} d k \int_{-\infty}^{\infty} \frac{p \sinh \pi p}{\cosh ^{2} \pi k+\sinh ^{2} \pi p} \\
& \quad \times \sqrt{\cos Y^{\prime} \cos Y^{\prime \prime}} P_{i k-\frac{1}{2}}^{i p}\left(\sin Y^{\prime \prime}\right) P_{i k^{-}-\frac{1}{2}}^{-i p}\left(\sin Y^{-\prime}\right) \epsilon^{i k\left(X^{\prime \prime}-X^{\prime}\right)} c^{-\frac{i T}{2 m}\left(p^{2}+\frac{1}{4}\right) .}
\end{aligned}
$$




\section{REFERENCES}

[1. V.Allessandrini, D.Amati, M.Le Bellac and D.I.Olive: The Operator Approach to Dual Multiparticle Theory; Physics Reports 1C (1971), 269.

[2] L.Alvarez-Gaumé, G.Moore and C.Vafa: Theta-Functions. Modular Invariance and Strings; Commun.Math.Phys. 106 (1986), 1 .

[3] K.Aoki: Heat hernels and Superdeterminants of Laplace Operators on Super Riemann Surfaces; Commun. Math. Phys. 117 (1988), 405;

K.Aoki and V.Periwal: Twisted Non-Abelian Determinants on Riemann Supersurfaces; University of California preprint, Los Angeles, NSF-ITP-88-166.

[4] K.Aoki: Private Communication.

[5] R.Aurich and F.Steiner: On the Periodic Orbits of a Strongly Chaotic System; Physica D 32 (1988), 451 .

[6] R.Aurich and F.Steiner: DESY preprint, to appear.

[7] R.Aurich, M.Sieber and F.Steiner: Quantum Chaos of the Hadamard-Gutzwiller Model; Phys. Rev. Lett. 61 (1988), 483

[8] N.L.Balazs and A.Voros: Chaos on the Pseudosphere; Physics Reports 143 (1986), 109

[9] A.M.Baranov, Yu.I.Maniu, I.Y. Frolov and A.S.Schwarz: The Multiloop Contribution in the Fermionic String; Sov.J.Nucl. Phys. 43 (1986), 670.

[10] A.M.Baranov, Yu.l.Manin, I.V.Frolov and A.S.Schwarz: A Superanalog of the Selberg Trace Formula and Multiloop Contributions for Fermionic Strings; Commun.Math.Phys. 111 (1987), 373.

[11] M.A.Baranov and A.S.Schwarz: Multiloop Contribution to String Theory; JETP Leti. 42

On the Multiloop Contribution to the String Theory; Int.J.Mod.Phys. A2 (1987), 1773

[12] A.A.Belavin and V.G.Knizhnik: Algebraic Geometry and the Geomelry of Quantum Strings; Phys.Lett. 168B (1986), 201.

[13] F.A.Berezin: Canonical Operator Transformation of Second Quantization; Soviet Physics Dokl B (1961), 212 .

[14] F.A.Berezin: Introduction to Superanalysis (Ed.A.A.Kirillov); (Reidel. Dordrecht, 1987).

[15] L.Bers: Finite Dimensional Teichmülier Spaces and Generalizations; Bull.Amer.Math.Soc. 5 (1981), 131

16] J.Botte and F.Steiner: Determinanis of Laplace-Like Operators on Riemann Surfaces: DESY preprint, DESY $88-190$

[17] L.Brink, P.DiVecchia and P.S.Howe; A Lucally Supersynmetric and Reparametrisation Invariant Action for the Spinning String; Phys.Lett. 65B (1976), 471.

[18: A.Comtet: On the Landau Levels on the Hyperbolir Plane; Ann.Phys.(N.Y.) 173 (1987), 185.

(19) S.Deser and B.Zumino: A Complete Action for the Spinning String; Phys.Lett. 65B (1976)

[20] B.de Wit, M.Lüscher and H.Nicolai: The Supermembrane is Unstable; DESY preprint, DESY $88-162$

[21] B.S.DeWitt: Dynamical Theory in Curved Spaces:1. A Review of the Classical and Quantum Action Principles; Rev.Mod.Phys. 29 (1957), 377.

[22] B.S. DeWitt: Supermanifolds; (Princeton University Press, Princeton, 1987).
23. E. D'Hoker and D. H. Phong: Multiloop Amplitudes for the Bosonic Polyakov String: Nucl. Phys. B280 (1986). 20:

On Determinants of Laplacians on Riemannian Surfaces: Commun.Math.Phys. $104(1986)$ 537.

24. E.D'Hoker and D.H.Phong: Loop Amplitudes for the Fermionic String; Nucl.Phys. B278 (1986). 225:

The Geometry of String Pertubation Theory; Princeton preprint. PUPT - 1039, to be published in Rev.Mod. Ptys. 60. (1988)

25. A.Erdelyi, W. Magnus, F. Oberhettinger, F.G. Triconi: Table of Integral Transforms Vol.I.; ( $M c$. (iraw, Hill, Neu York, 1985).

26. R.Fenn: What is the Geometry of a Surface; Amer. Math.Monthly $90(1983), 87$

27. R.Feynman and A.Hibbs: Quantum Mechanics and Path Integrais; (McGraw Hill. New York, 1965).

28. P. Frampton: Dual Resonance Models; (Benjamin. 1974)

$29^{2}$ P.G.O. Freund and M.Olson: Non Archimedean Strings: Phys.Lett. 199B (1987). 186 P-Adic Dynamical Systems: Nucl. Phys. B287 (1988), 86:

P.G.O.Fremid and F. Witten: Adelic String Amplitude: Phys. Lett. 199B (1987). 191.

30. D. Friedan: Introduction to Polyakov's String Theory; Les Houches, Session XXXIX, 1984, Eds. J.-B. Zuber and R.Stora. p. 839

31: G.Gilbert: String Theory Path integral - Genus Two and Higher; Nucl.Phys. B277 (1986),

32. F.Gliozzi, J.Scherk and D.Olive: Supergravity and the Spinor Dual Model; Phys.Lett. 65B (1976), 282;

Supersynametry. Supergravity Theories and the Dual Spinor Model; Nucl.Phys. B122 (1977), 253.

33i I.S.Gradshteyn, I.M.Ryzhik: Table of Integrals, Series and Products; (Academic Press, 1980).

34: M.B.Green: Supersymmetrical Dual String Theories and Their Field Theory Limits; Survey in High Energy Physics 3 (1983). 12

[35. M.B.Green and J.H.Schwarz: Supersymmetrical Dual String Theory (1): Nucl.Phys. B181 (1982). 502:

Supersymmetrical Dual String Theory (II), Vertices and Trees; Nucl. Phys. B188 (1982), 252; Supersymmetrical Dual String Theory (III), Loops and Renormalization; Nucl. Phys, B188 (1982), 441.

36. M.B.Green and J.H.Schwarz: Anomaly Cancellations in Supersymmetric $D=10$ Gauge Theor and Superstring Theory; Phys. Lett. 149B (1984), 117;

Infinity Cancellations in $S O(32)$ Superstring Theory; Phys.Lett. 151B (1985), 21; The Hexagon Gauge Anomaly in Type I Superstring Theory; Nucl.Phys. B255 (1985), 93

37 . M.B.Green, J.H.Schwarz and E. Witten: Superstring Theory I, II; (Cambridge University Press. (ambridge. 1987).

[38] C.Grosche: The Product Form for Path Integrals on Curved Manifolds; Phys.Lett. 128A (1988), 113.

[39] C.Grosche: The Path Integral on the Poincaré Upper Half-Plane witl a Magmetic Field and for the Morse Potential; Ann.Phys.(N.Y.) 187 (1988), 110

40) C.Grosche: The Path Integral on the Poincaré Disc, Poincaré $\mathrm{C}$ pper Half-Plane and the Hyperbolic Strip: DESY preprint DESY 88 - 074; submitted to J.Math.Phys.

[41: C.Grosche and F.Steiner: The Path Integral on the Poincaré Upper Half Plane and for Liouville Quant un Mechanics; Phys.Lett. 123A (1987), 319

142. C.Grosche and F.Steiner: Path Integrals on Curved Manifolds: Z.Phys. C $36(1987), 699$ 
[43] C.Grosche and F.Steiner: The Path Integral on the Pseudosphere; Ann.Phys.(N.Y.) 282 (1988), 120 .

[44] D.J.Gross, A.Harvey, E.Martinec and R.Rohm: Heterotic String Theory (I): The Free Heterotic String; Nucl. Phys. B256 (1985), 253;

Heterotic String Theory (II): The Interacting Heterotic String; Nucl.Phys. B287 (1986), 75.

[45] D.J.Gross and V.Periwal: String Pertubation Theory Diverges; Phys, Rew.Lett. 60 (1988) 2105

[46] M.C.Gutzwiller: Geometry of Quantum Chaos; Phys.Scripta 8 (1985), 184.

[47] S.Hawking: Zeta Function Reguralization of Path Integrals in Curved Spacetime; Commun. Math.Phys. 55 (1977), 133.

[48] S.Hawking: Is the End in Sight for Theoretical Physics? CERN Courier 21 (1981), 3 (Part.1), CERN Courier 21 (1981), 71 (Part.2), (Inaugural lecture).

[49] D. Hejhal: The Selberg Trace Formula for PSL(2,R), Vol. I; Lecture Notes in Mathematics 548; (Springer-Verlag, Berlin 1976).

50] P.S. Howe: Superspace and the Spinning String; Phys.Lett. 70B (1977), 453.

[51] P.S.Howe: Super Weyl Transformations in Two Dimensions; J. Phys. A12 (1979), 393.

[52] M.Jacob (Ed.): Dual Theory; (Physics Reports Reprint Vol.I, North-Holland, Amsterdam, 1974).

[53] A.Jevicki; Lectures on Interacting String Field Theory; 5th Adriatic Meeting on Particle Physics: Superstrings, Anomalies and Unification; Eds.: M.Martinis and I. Andrić; (World Scientific, 1987).

[54] R.Kubo: Path Integration on the Upper Half-Plane; Prog.Theor. Phys. 78 (1987), 755; Geometry, Heat Equation and Path Integrals on the Poincaré Upper Half-Plane; Prog. Theor. Phys. 78 (1988), 217.

55] W.Magnus, F.Oberhettinger and R.P.Soni: Formulas and Theorems for the Special Function of Theoretical Physics; (Springer-Verlag, Berlin, 1966).

[56] S.Mandelstam: Dual Resonance Model; Physics Reporis 13C (1974), 259.

[57] S.Mandelstam: Interacting String Picture of Dual-Resonance Models: Nucl.Phys. B84 (1973),

Interacting String Picture of the Nevell-Schwarz Model; Nucl.Phys. B68 (1974), $7 \tau$.

[58] Yu.l.Manin: The Partition Function of the Polyakov String can be expressed in Terms of Theta-Functions; Phys. Lett. 172B (1986), 184.

59? I.L.Martin: Generalized (lassical Dynanics, and the "C'lassical Analogue" of a Fermi Oscillator Proc.Roy.Soc. A251 (1959), 536:

The Feynamn Primciple for a Fermi System: Proc.Roy.Soc. A251 (1959), 543.

60 S.Matsumoto and Y.Yasui: ('haos on the Super Riemam Surface; Prog.Theor. Phys. (1988), 1022.

61: S.Matsumoto. S.I ehara and Y.Yasui: Hadanard Model on Super Riemann Surface; NOR DITA preprint. NORDI'TA-88/33 P.

62 H.P.Mchean: Selherg's Trace Formula as Applied to a Compact Riemamn Surface: Com mun. Pure and Aprl.Math. 25 (1972). 225.

63: G.Moore, P.Nelson and J.Polchinski: Strings and Supermoduli: Phys.Leti. 189B (1986), 4i.

64 M.A.Namazie and S.Rajiev: On Multiloop Compulations in Polvakov's String Theory: Nucl. Phys B277 (1986). 332.

65. A.Neveu and J.H.Scliwarz: Quark Model of Dual Pious: Phys. Rev. D4 (1971), 1109.
[66] H.Ninnemann: Klassische and Quantennechanische Bewegung auf Super Riemannschen Flächen; Sonmersemester 1988 Universität Hamburg: Seminar über hyperbolische Geometrie und Anwendungen in der Physik.

[67] M.Omote: Point Canonical Transformations and the Path Integral; Nucl. Phys. B120 (1977), 325.

[68] M.Omote and H.Sato: Quantum Mechanics of a Non Linear System; Progr.Theor.Phys. 47 (1972), 1367.

[69] D.Peak and Inomata: Summation Over Feynman Histories in Polar Coordinates; J.Math. Phys. $10(1969), 1422$.

[70] A.M.Polyakov: Quantum Geometry of Bosonic Strings; Phys.Lett. 103B (1981), 207; Quantum Geometry of Fermionic Strings; Phys.Lett. 103B (1981), 211; Gauge Fields and Strings; (Harwood Academic Publishers, Chur, 1987).

[71] J.M.Rabin and L.Crane: Global Properties of Supermanifolds; Commun.Math.Phys. 100 (1985), 141;

How Different are the Supermanifolds of Rogers and DeWitt?; Commun Math Phys 102 (1985), 123;

Super Riemann Surfaces: Uniformization and Teichmüller Theory; Commun.Math.Phys. 113 (1988), 601.

[72! P.Ramond: Dual Theory for Free Fermions; Phys.Rev. D3 (1971), 2415.

[73] D.Ray and I.M.Singer: Analytic Torsion for Complex Manifolds; Ann.Math. 98 (1973), 154

[74] C.Rebbi: Dual Models and Relativistic Quantum Strings; Physics Reports 9C (1974), 1.

[75] M.Reed and B.Simon: Methods of Modern Mathematical Physics Vol.II; (Academic Press, New York, 1975).

[76] A.Rogers: A Global Theory of Supermanifolds; J.Math. Phys. 21 (1980), 1352; On the Existence of Global Integral Forms on Supermanifolds; J.Math. Phys. 28 (1985), 2749; Graded Manifolds, Supermanifolds and Infinite-Dimensional Grassmann Algebras; Commun. Math.Phys. 105 (1986), 375.

[7i] J.Scherk: An Introduction to the Theory of Dual Models and S1rings: Rev,Mod.Phys. 47 $(1975), 123$.

[78] J.Scherk and J.H.Schwarz; Dual Models for Non Hadrons: Nucl. Phys. B81 (19;4), 118.

[79: J.H.Schwarz: Dual Resonance Model; Phys.Reports 8C (19i3). 269

80: J.H.Schwarz: Superstring Theory; Phys.Reports 89 (1982), 223.

81 J.Schwinger: A Note on the Quantization Dynamical Principle: Phil.Mag. 44 (1953), 1171; The Theory of Quantized Fields IV: Phys, Rev. 02 (1953), 1283.

82. A.Selberg: Harmonic Analysis and Discontinuous Groups in Weakly Riemannn Spaces with Applications io Dirichlet Series; ..Indian Math.Soc. 20 (1956). $4 \hat{i}$.

i83) W.Siegel and B.Zwiebach: Giauge Strings Fields; Nucl.Phys. B263 (1986), 105;

W.Siegel: String Field Theory via BRST; Unified String Theories, eds.: M.Green and D.Gross: (Santa Babara. $398 \pi$ )

B.Zwiebach: Gauge lnvariant String Actions; ibidem.

84. F. Steiner: On Selberg's Zeta Function for Compact Riemann Surfaces; Phys. Lett. 188B 1986 .

Quantum Chaos and Geometry: "Recent Developments in Mathematical Physics". Conference Schladming 198ī, p.305 (Eds.: H.Mitter, L.Pittner); (Springer-Verlag, Berlin, 1987).

85. A. Terras: Noneuclidean Harmonic Analysis: SIAM Reniew 24 (1982), 159;

Harmonic Analysis on Symmetric Spaces and Applications I: (Spronger-Verlay. New Sork. 1987 
86. S. Y'ehara and Y.Yasui: A Superparticle on the "Super" Poincaré Upper Half-plane; Phys.Lett. 202B (1988), 530 .

[87] S.Uehara and Y.Yasui: "Super" Selberg Trace Formula from Chaotic Mode]; King's College London preprint $198 \mathrm{i}$

[88] E.Verlinde: Fusion Rules and Modular Transformations in 2-Dimensional Conformal Field Theory; Nucl. Phys. 300 (1988), 360.

[89] G.Veneziano: An Introduction to Dual Models of Strong Interaction and their Physical Motivations; Physics Reports 8C (1974), 199.

[90] G.Veneziano: A String Theory Needs Just Two Constants; Europhys. Lett. $2(1986), 199$

(91) H.A.Vilenkin: Special Functions and the Theory of Group Representations; ( Am.Math. Soc.Providence, Rhode Island 1968).

[92] I.V.Volovich: P-Adic Strings: Class.Quant.Grav. 4 (1987). L83.

P-Adic Space-Time and String Theory: Theor.Math.Fiz. 71 (1987), L574 (Engl.);

Harmonic Analysis and P-Adic Strings; Lett.Math.Phys. 18 (1988), 61.

[93] G.B.West: An Introduction to String Field Theory: A Pedestrian Approach to the Covarian Formulation; Aspen Winter Conf. (1986), 277.

94] E.Witten: Non-Commutative Geometry and String Field Theory; Nucl.Phys. B268 (1986)

[95] S.A.Wolpert: Asymptotics of the Spectrum and the Selberg Zeta-Function on the Space of Riemann Surfaces; Commun. Math.Phys. 112 (1987), 283. 